\title{
Role of Perivascular Adipose Tissue in Vascular Pathology and the Therapeutic Effect of Exercise
}

\author{
Evan Ray DeVallance
}

Follow this and additional works at: https://researchrepository.wvu.edu/etd

\section{Recommended Citation}

DeVallance, Evan Ray, "Role of Perivascular Adipose Tissue in Vascular Pathology and the Therapeutic Effect of Exercise" (2017). Graduate Theses, Dissertations, and Problem Reports. 5481.

https://researchrepository.wvu.edu/etd/5481

This Dissertation is protected by copyright and/or related rights. It has been brought to you by the The Research Repository @ WVU with permission from the rights-holder(s). You are free to use this Dissertation in any way that is permitted by the copyright and related rights legislation that applies to your use. For other uses you must obtain permission from the rights-holder(s) directly, unless additional rights are indicated by a Creative Commons license in the record and/ or on the work itself. This Dissertation has been accepted for inclusion in WVU Graduate Theses, Dissertations, and Problem Reports collection by an authorized administrator of The Research Repository @ WVU.

For more information, please contact researchrepository@mail.wvu.edu. 
Role of Perivascular Adipose Tissue in Vascular Pathology and the Therapeutic Effect of Exercise

Evan Ray DeVallance

Dissertation submitted to the School of Medicine at West Virginia University in partial fulfillment of the requirements for the degree of:

Doctor of Philosophy in Exercise Physiology

Dissertation Committee:

Paul D. Chantler, Ph.D., Committee Chair/ Advisor

Jefferson C. Frisbee, Ph.D.

I. Mark Olfert, Ph.D.

Emidio Pistilli, Ph.D.

Stephen Alway, Ph.D.

David Siderovski, Ph.D.

Department of Exercise Physiology

Morgantown, WV 2017

Key Words: metabolic syndrome, endothelial dysfunction, perivascular adipose tissue, exercise, depression, oxidative stress, inflammation

Copyright 2017, Evan DeVallance 
Abstract

\title{
Role of Perivascular Adipose Tissue in Vascular Pathology and the Therapeutic Effect of Exercise
}

\author{
Evan R. DeVallance
}

Aortic compliance is important for dampening pulsatile flow and delivery of continuous flow to the periphery. Aortic compliance is regulated by extracellular matrix composition and endothelial derived nitric oxide. Disruption of aortic endothelium or extracellular matrix can lead to the development of aortic stiffness an independent risk factor of cardiac events and mortality. Over the past decade perivascular adipose tissue (PVAT) surrounding the vasculature has come to light as an important regulator of artery function. The main focus of this dissertation is to evaluate the role PVAT surrounding the aorta in mediating endothelial relaxation in health and disease. Specifically, metabolic syndrome (MetS) and chronic stress induced depressive states.

The United States has a high prevalence of both MetS and depressive states, 34\% and $17 \%$ respectively. Additionally, the co-prevalence of depressive states with MetS is common. Both MetS and depressive states are associated with vascular dysfunction, in part, mediated through an increase of pro-inflammatory cytokines and oxidative stress. Little is known about the impact of MetS on PVAT regulation of the aorta and the impact of depressive states or depressive states concomitant with MetS on PVAT is unexplored.

Aerobic exercise training is recognized to have antioxidant and anti-inflammatory properties and promotes vascular health. Exercise training has been shown to reduce aortic stiffness and reduce the risk of cardiac events and mortality. Likewise exercise treatment of MetS or depressive state yields beneficial effects and limits the vascular pathology of these disease state. However, the actions of exercise on PVAT in health and disease are poorly understood. In order to evaluate these gaps in knowledge, I purpose the following specific aims for this dissertation:

- Determine Metabolic Syndrome's impact on Thoracic Aorta PVAT and PVAT derived TNF $\alpha$ contribution to aortic dysfunction

- Determine if UCMS impacts the regulation of PVAT on aortic function

- Test the therapeutic effectiveness of aerobic exercise on PVAT and its regulation of aortic function

The results of this study will establish the role of PVAT in mediating aortic dysfunction associated with MetS and depressive state. Additionally, this work will identify key disease specific mediators of PVAT regulation of aortic function. Finally, this work will establish mechanisms through which exercise mediates beneficial aortic function. 


\section{Dedication}

To my beautiful wife Nicole DeVallance and our sons William and Lucas for your support and the joy you bring.

To all, currently with me and who have passed on that have shown support over my academic journey.

To all the obstacles in my way that make this moment that much sweeter.

To West Virginia may country roads bring me home in the future. 


\section{Acknowledgements}

So many people have directed my academic path. I would like to thank everyone as this dissertation would not be possible without them.

To my parents, grandparents, sister, aunts, and uncles thank you for all your support and guidance. Thank you for leading by example with great work ethics and driving me to always be my best regardless of the task, whether it be cleaning a room or completing a dissertation.

Doug Harney, for stirring my interest in exercise and teaching me most of what I know about exercise training.

Greg Lahman, for being a great mentor and teaching me about the sport specific design of exercise programs. Showing confidence in me and allowing me to develop and implement programs for the various sport teams. This internship allowed by to interact with some amazing people, Joe Tiller, Matt Painter, Ryan Kerrigan, JaJuan Johnson, Robbie Hummel, Chris Kramer, E’Twaun Moore, and Nemanja Calasan.

To Steven McKenzie, at Purdue for believing in me and being the only faculty member to write me a letter of recommendation. 
Coach Duane Potts and Scott Ulhorn, for picking me up when I was done. Scott Ulhorn suggested that I help them coach high school football. After losing the 2 graduate assistant positions for strength and conditioning I was pretty down and my future was uncertain. Coach Potts and the team's parents club raised money to pay me as a strength coach and positions coach during the year I had off between undergrad and grad school. Coach Potts lifted my confidence by believing in me and letting me take over all strength and conditioning duties. This simple support gave me the confidence to peruse further education.

To my cousin Dave for nagging me to apply to WVU on what seemed to be a weekly basis. For being the first one in the family to get a $\mathrm{PhD} \mathrm{I}$ am merely following in his footsteps. He encouraged me to continue after my masters and get my PhD. Helped support me along the way from being the best man at my wedding, discussing the research process and helping guide me through my $\mathrm{PhD}$, to hanging out and grabbing a beer at the apothecary.

To all the HPL staff: Danny, Dave, Diana, Lori, and Jim. For teaching me everything I know about clinical exercise physiology. As well as being friendly people to spend the day with helping clients in the HPL and sharing life's successes. Additional to Dave and Jim for talking hunting.

To the EXPH Master selection committee I know my application packet was underwhelming, I thank you for giving me a chance. To Dr. Alway for scraping up 
funding, following my two years in the master's program to support a bridge year, so I could be competitive for the $\mathrm{PhD}$ program.

Dr. Bryner for being a great teacher. Expanding my critical thinking skills from conversations about various research topics. Also for bringing me into my first research project studying the effects of chronic stress and wheeling running on bladder cancer.

Dr. Shaller: For what I thought at the time was a horrible set back in my career. I can still here it now, "I just don't think you have enough research experience, you might benefit from working as a tech and applying again next". However, I probably have that moment to thank for setting my success in the PhD program. I also remember him asking if I want coffee and me responding with "I only drink it on the weekends". He looked at me and laughed and said that will change sure enough he was right on that account as well.

To Dr. Minnear for giving me a list of what my short coming were as an applicant. With the motivation of rejection and a clear path to admittance insight I got to work. Upon reapplying the following year Dr. Minnear recommended me for in-house fellowships and I received the STEM Mountains of Excellence Fellowship to support the first 3 years of my PhD. Also, a big thanks to Dr. Minnear and the instructors involved for allowing me to come back after 4 weeks of being restricted to bed due the complete rupture of my Achilles tendon, and then take 6 exams over the course of 2 weeks to stay on track and keep my fellowship. Oh, and a big thanks to Lyme disease for making this happen. 
Alex Tylka for taking over the burden of me writing 12 months of a workout program for myself. Our lifting sessions were always a nice break from the day. Best of luck in your new faculty position at Miami of Ohio University.

Vincent Setola for teaching me a lot about science in general and pushing me (figurative and literally while I was in my wheelchair) to be my best. Also for teaching me the finer points of French wine and for discussing wine, French, and Italian culture. For his continued helpfulness, even though I joined the Chantler lab.

To Dr. Siderovski for giving me the opportunity to rotate in your lab and extending your resources to aid in the completion of my dissertation work. Furthermore, more being a part of my committee even though could pursue RGS component of in my project.

To Dr. Pistilli for opening his lab when I approached him with an idea to use his equipment, even though I had no evidence it would work. I thank you for working with me to make this idea work and I believe it is a key component of the work presented in this dissertation.

The Olfert lab. For sharing your office and many grueling hours in lab in various collaborative efforts. Kyle for sharing my love of hot sauce and education of TSP-1. Thank you to Matt, Hanna, Chris for all your help. 
Jeff and his lab. Jeff for his willingness to help and his open discussion of ideas. For his dislike of the PVAT topic as it pushed me to read the literature and gain the knowledge needed to have a constructive discussion about the topic. To Josh for teaching me how to work with animals and hang micro vessels. To Steve for suffering through the seemingly endless days of the EP study and being engaging scientifically and for our general amusement. I guess I will include Kent as part of this as well since he abandoned country to join Jeff's lab in Canada. To Kent for all his hard work on the EP study and directly working on parts of my study, alternating staying late so I could spend time with my family.

To Paul for all his assistance from introducing me to clinical research and transitioning into the PhD program. Teaching me all I know at statistics. Additionally, for showing faith in me to pick my own research topic. I have truly enjoyed working for you and look forward to the potential for collaborative efforts in the future.

To my lab mates past and present;

To Miriam and Brain for being great friends and supporting me during our time in the master program together.

Sara Fournier: For teaching me how to conduct all of the clinical experiments to even exvivo aortic reactivity. I learned a lot from you.

Kayla Branyan: For putting up with me over the past 4 years as we were stuck in lab a lot together. You were always helpful and together I think we ran the lab pretty well. I hope 
I was half as helpful to you as you were for me. You have accomplished a lot after moving into the lab I wish all the best of luck as you head to California.

Chris Skinner for keeping me up to date on pop culture and for your hard work in the lab even with a GA position in wellness. It's been fun playing trivia with you Kayla and Adam. Best of luck in finishing your PhD.

Shin: for teaching me new techniques and being a sounding board for me to bounce ideas off of.

Luca: For being a good friend and working hard in the lab. But most of all for showing interest in my son William and interacting with him. Also for introducing me to biscotti dipped in red wine its simply amazing, can't wait to visit you in Italy. Fino ad allora mio amico

To all the interns who have rotated through the lab and contributed to this work I thank you. I could not even begin to list all of your names, but I would like to highlight the work of Clinton, Whitney, Catherine, and Ashlee.

Finally, to my beautiful wife Nicole for uprooting your life in Bloomington and moving to Morgantown, West Virginia. You came along kicking and screaming, but now we have spent most of our lives together here and I'm sad to uproot you again and drag you to Pittsburgh. You are wonderful and understanding of the career path I have chosen, and I know I can always count on you to support me. You are an amazing mother and William is and Lucas will be luck to spend their day with you. You have even contributed to much 
of lab work from staying late with me and helping to clean to reading over papers or helping me study. My success in my academic career was dependent on your unwavering support.

To William for always brightening my day when I'm feeling down. You seem to have a way to sense when we need a pick up and you always deliver. I know you will face many challenges in your life and I hope I can return the favor and help you through them, to both Lucas and William I can't wait to spend every free second, I have with you. 
Table of Contents

DEDICATION III

ACKNOWLEDGEMENTS IV

CHAPTER 1: REVIEW OF LITERATURE

SUMMARY AND PURPOSE OF THE DISSERTATION 29

CHAPTER 2: METHODS

TPVAT PILOT DATA $\quad 63$

CHAPTER 3: AORTIC DYSFUNCTION IN METABOLIC SYNDROME MEDIATED BY

PERIVASCULAR ADIPOSE TISSUE TNFA AND NOX2 DEPENDENT PATHWAY 66

INTRODUCTION $\quad 69$

METHODS \& MATERIALS $\quad 70$

RESULTS $\quad 77$

DISCUSSION

REFERENCES $\quad 91$

$\begin{array}{ll}\text { FIGURES } & 103\end{array}$

CHAPTER 4: AEROBIC EXERCISE PREVENTS THE IMPAIRMENT OF AORTIC FUNCTION IN METABOLIC SYNDROME: ROLE OF PERIVASCULAR ADIPOSE TISSUE.

INTRODUCTION $\quad 117$

$\begin{array}{ll}\text { METHODS } & 118\end{array}$

RESULTS $\quad 123$

DISCUSSION 127

REFERENCES

$\begin{array}{ll}\text { FIGURES } & 145\end{array}$

CHAPTER 5: CHRONIC MILD STRESS INDUCED PERIVASCULAR ADIPOSE TISSUE IMPAIRMENT OF AORTIC FUNCTION AND THE THERAPEUTIC EFFECT OF EXERCISE IN LEAN AND OBESE ZUCKER RATS.

INTRODUCTION: $\quad 153$

METHODS 154

RESULTS: $\quad 159$

DISCUSSION: $\quad 165$

REFERENCES $\quad 174$

$\begin{array}{ll}\text { FIGURES } & 185\end{array}$

CHAPTER 6: DISSERTATION DISCUSSION

SUMMARY $\quad 197$

FUTURE DIRECTION $\quad 202$

LONG TERM OUTLOOK $\quad 204$ 


\section{List of Figures}

Figure 1.1. Digital representation of arterial wall with perivascular adipose tissue ............2

Figure 1.2. Image of obesity induced PVAT dysfunction ................................................ 13

Figure 1.3. Depiction of known and potential PVAT regulation of vascular function. .... 21

Figure 2.1. 200mg Verification ........................................................................................ 63

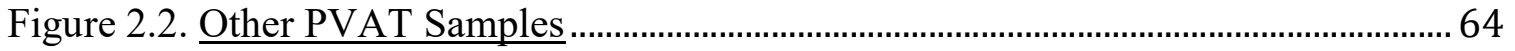

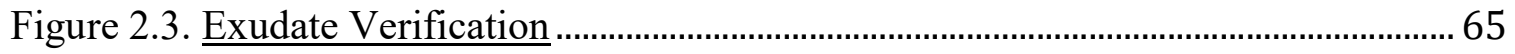

Figure 3.1. Relative gene expression of tPVAT ………………………………………....103

Figure 3.2. tPVAT ROS formation SOD defense and proteasome function.....................104

Figure 3.3.Cytokine profile of tPVAT............................................................................105

Figure 3.4. OZR-tPVAT role in activating aortic ROS production and reducing nitric

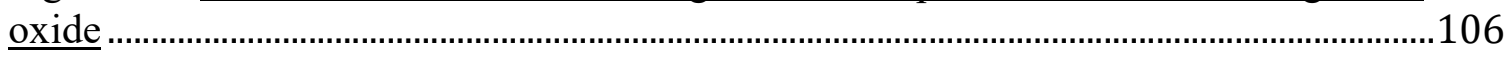

Figure 3.5. Effect of TNF $\alpha$-nAB on tPVAT and tPVAT mediated aortic function .........107

Figure 3.6. Role of tPVAT in aortic stiffness ....................................................................108

Figure 4.1. tPVAT Reactive Oxygen Species and Phenotype ............................................145

Figure 4.2. Ex Enhancement of Proteasome Function.....................................................146

Figure 4.3. tPVAT Cytokine Profile Following Ex Figure 4.4. Effect of Ex on tPVAT

Mediated Aortic Function .................................................................................................147

Figure 5.1. Effect of UCMS and Exercise on Aortic Relaxation......................................185

Figure 5.2. Effect of UCMS and Exercise on Nitric Oxide and Aortic Reactive Oxygen

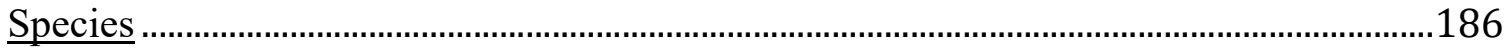

Figure 5.3. Effect of UCMS and Exercise on tPVAT Reactive Oxygen Species and tPVAT Immuno-Attractant Release ..................................................................................187

Figure 5.4. Effect of UCMS and Exercise on tPVAT Cytokines .......................................188

Figure 5.5. Effect of UCMS and Exercise on TPVAT Angiotensin II and Aldosterone .189

Figure 5.6. Effect of UCMS and Exercise Prevents Aortic Stiffness................................190 


\section{$\underline{\text { List of Tables }}$}

Table 4.1. Body Weight, Blood Pressure, and Blood Profile of LZR and OZR

Table 5.1. Assessment of Body Weight, Blood Pressure, Blood Profile, \& $\underline{\text { Stress Indices }}$

Table 6.1. Effect of tPVAT on Aortic Function

Table 6.2. Effect of tPVAT on Aortic Function 
Abbreviations

4-Amino-5-Methylamino-2',7'-Difluorofluorescein-Diacetate

(DAF-FM-DA)

4-Hydroxy-TEMPO

(TEMPOL)

Aerobic Exercise Training

$(\mathrm{AET})$

Chemokine (C-X-C motif) ligand-1

(KC/Gro)

Dihydroethidium

(DHE)

Endothelial Dependent Dilation

(EDD)

Extracellular Matrix

(ECM)

Interferon Gamma

$(\mathrm{IFN}-\gamma)$

Interleukin

Lean Zucker Rat

(LZR)

Matrix Metalloproteinase

(MMP)

Metabolic Syndrome

(MetS)

Monocyte Chemoattractant Protein-1

(MCP-1)

NADPH-Oxidase 2

(NOX2)

Nitric Oxide

(NO)

Nitro-L-Arginine Methyl Ester

(L-NAME)

Nuclear Factor (Erythroid-Derived 2)-Like 2

$(\mathrm{Nrf} 2)$

Nuclear Factor Kappa-Light-Chain-Enhancer

$(\mathrm{NF}-\kappa \mathrm{B})$

Obese Zucker Rat

(OZR)

Optimal Cutting Temperature Compound

(OCT)

Perivascular Adipose Tissue

(PVAT) 
Polymerase Chain Reaction

(PCR)

Reactive Oxygen Species

(ROS)

Sodium Nitroprusside

(SNP)

Superoxide Dismutase

(SOD)

Thoracic Perivascular Adipose Tissue

(tPVAT)

Thrombospondin 1

(TSP-1)

Tissue Inhibitor of Metalloproteinase

(TIMP)

TNF $\alpha$ Neutralizing Antibody

(TNF $\alpha-n A B)$

Tumor Necrosis Factor Alpha

(TNF $\alpha)$

Uncoupling Protein-1

(UCP-1)

Unpredictable Chronic Mild Stress

(UCMS) 


\section{Chapter 1: Review of Literature}

\section{Aortic Function}

The aorta is the largest artery in the body and directly receives blood from the heart and helps distribute blood to most of the body. The aortic wall is comprised of 3 regions the tunica intima, tunica media, and tunica adventitia. The tunica intima consists of a single interconnected layer of endothelial cells with underlying extra cellular matrix (ECM). The endothelium provides a physical barrier and is responsible for regulation of function stiffness of the aorta. The internal elastin lamina separates the tunica intima from the tunica media. The tunica media is made up of alternating circumferentially organized layers of elastin and smooth muscle with interspersed elastin, collagen, and proteoglycans. This layer is the major determinant of aortic distensibility. Finally, the external elastic lamina divides the tunica media from the tunica adventitia. The tunica adventitia consists primarily of collagen fibers conferring a rigid structure, with diffuse smooth muscle precursors, elastin, and vasa vasorum. The vasa vasorum is the local supply of blood to the aortic wall, which can traverse from outside the tunica adventitia almost to the tunica intima.

Due to the length of the aorta distinct anatomical regions segment the aorta with profound differences arising as you move from the thoracic aorta down into the abdominal aorta. The most notable difference is the ECM make up for the aortic wall with regions proximal to the heart presenting with higher levels of elastin in comparison to increasing collagen as you move distally from the heart [1]. The review will focus on the structure and function of the thoracic aorta as its 
function as a more direct impact on cardiac function, all mentions of aorta will refer to the thoracic region unless otherwise specified.

Figure 1.1. Digital representation of arterial wall with perivascular adipose tissue.

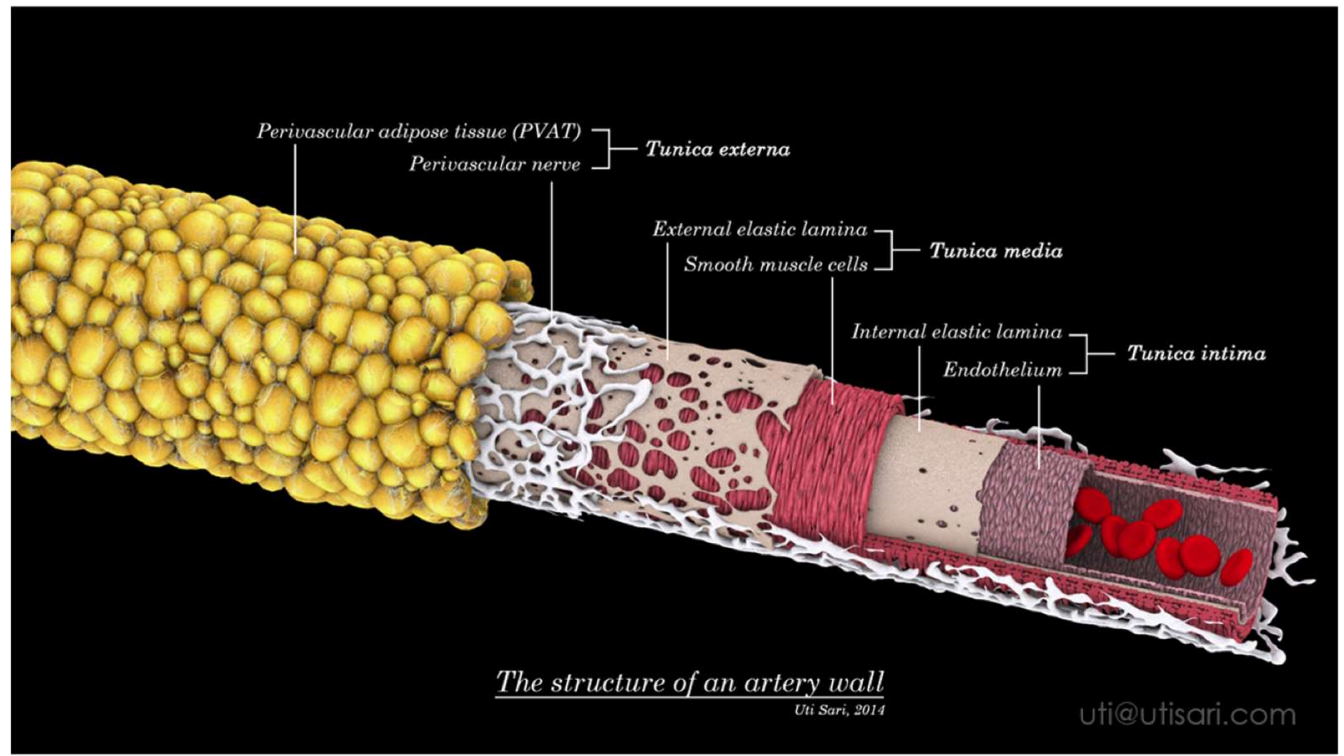

\section{Mechanical Properties of the Aorta}

The elastin is mainly organized in the medial layer of the aorta wall inter mixed with smooth muscle cells and collagen fibers. The high levels of elastin in the aorta give it elastic properties. The elastin properties allow the aorta to comply with high systolic pressures storing kinetic energy and blood to then recoil during cardiac diastole. This is known as the Windkessel effect and is important for the following reasons. It dampens the pulsatile nature of the cardiac flow and allows for continuous blood flow to the organs $[2,3]$. In the arch this same effect is needed to develop diastolic pressure and perfuse the coronary arteries as blood flow into the myocardium is greatly reduced during systole [4]. Secondly, the compliant nature of the aorta 
helps to maintain lower afterload allowing for more efficient cardiac work. Any change in the elastic properties of the aorta could negatively impact the heart and other organs. The media layer of the aorta also contains collagen fibers and smooth muscles. The collagen fibers help give the aorta its structure and become engaged as pressure increases inhibiting aortic distension. The aortic smooth muscle cells play more of a secretory role, making the elastin and collagen fiber building blocks $[5,6]$ and ECM remodeling proteins [7]. The prominent ECM remodeling proteins are matrix metalloproteinases (MMPs), which play an important role in normalizing shear stress via wall remodeling [8]. Additionally, transmembrane attachment of MMPs to the ECM allow the contractile state of the smooth muscles to differentially direct stress onto collagen and elastin fibers [9]. Therefore, making endothelial derived smooth muscle mediators important in the regulation of aortic compliance. The predominant smooth muscle modulator in the aorta is nitric oxide (NO), causing relaxation and preferential loading of elastin fibers while reduced NO and increased smooth muscle tone loads collagen [10,11]. The role of NO in aortic compliance is supported by decreases in aortic compliance with eNOS inhibition in mice [12]. This is in part due to impaired relaxation increasing tone of aortic smooth muscles exposing them to higher imparted wall stress leading to ECM remodeling to normalize the distribution of stress. Additionally, NO regulation of aortic compliance may be mediated through effects on remodeling proteins $[13,14]$. Gurjar et al. [13] showed eNOS gene transfer or NO donor compounds significantly reduced smooth muscle migration. This was mediated through upregulated levels of TIMP-2, which prevented the activation of both MMP2 and MMP9 to their active forms. This suggest NO mediates aortic compliance through regulation of smooth muscle tone causing the preferential loading of elastin fibers and protects the elastin fibers from degradation through TIMP sequestration of the elastases MMP2 and MMP9. 


\section{Disease Burden of Aortic Alterations}

In pathological conditions resulting in reduced NO or changes in ECM composition of the aorta stand to have significant health burdens. The loss of compliance in the aorta is deemed aortic stiffening. Stiffening refers to loss of elastic properties compared to the healthy norm resulting in a diminishing Windkessel effect and an increase of pulsatile flow [4]. Stiffness and pulsatile flow has numerous physiological effects on the heart and the periphery. At the heart, aortic stiffness increases afterload, work of the heart, and increases $\mathrm{O}_{2}$ demand [15]. While pulsatile flow reduces diastolic BP and coronary perfusion potentially resulting in $\mathrm{O}_{2}$ supply demand mismatch and a limited functional capacity $[4,16,17]$. One standard deviation increase in aortic stiffness, measured by aortic pulse wave velocity, elevates the risk of cardiac events and risk cardiac mortality $\sim 50 \%$ [18]. Systemically, pulsatile flow disrupts smooth continuous flow in the capillaries hampering capillary-tissue exchange [19]. Aortic stiffness is associated with microvessel damage and organ damage leading to pathology. Making aortic stiffness an independent risk factor for stroke, stroke mortality, all-cause mortality [20, 21].

\section{Perivascular Adipose Tissue Modulation of Vascular Function}

For the longest time the adipose tissue sitting outside of the vascular wall adventitia was thought to act simply as a cushion (Fig.1.1). However, over the past decade the perivascular adipose tissue (PVAT) has been recognized as an important regulator of vascular function. PVAT has been shown to release factors, which can modulate ECM, smooth muscle, and endothelial cells. Importantly, PVAT appears to have functions distinct from other depots of adipose and 
alterations in normal PVAT function have been recognized as key mediators of disease progression. The PVAT depot of adipose appears to be distinct from other depots and regulate vascular function based on the following evidence.

\section{Origin and Regionality of Perivascular Adipose Tissue}

Proteomic and genomic profile assessment of PVAT shows close similarity to brown adipose and distinct differences in comparison to white adipose tissue (visceral, subcutaneous, etc.) [22]. Despite similarities to brown adipose PVAT likely develops from a separate lineage. This was first discovered crossing peroxisome proliferator-activated receptor- $\gamma(\operatorname{PPAR} \gamma)$ floxed mice with smooth muscle specific driver SM22 $\alpha$ [23]. PPAR $\gamma$ is an essential regulator in adipogenesis, lipid storage, and lipid metabolism [24]. The conditional knock of PPAR $\gamma$ from smooth muscle precursors results in the development a vasculature devoid of PVAT [23]. To the contrary, brown and white adipose depots developed normally due to separate developmental origin of lineages $[25,26]$. The idea of PVAT developing alongside smooth muscle from a common origin bares similarities to the development of brown adipose which develops from the same precursors as skeletal muscle [27]. Not only does it appear PVAT is its own unique adipose depot but it displays various phenotypes and function depending on location (Fig.1.2) [28]. The PVAT surrounding the thoracic aorta (tPVAT) is unique as it's the only brown-like depot with a very dense population of mitochondria. The abdominal aorta transitions to a beige phenotype with a mix of brown and white like cells. Finally, mesenteric and the majority of peripheral PVAT presents with a white-like phenotype. These phenotypic differences along the vasculature correspond with variations in adipokine/hormone expression level [29]. More evidence in whitelike PVAT shows adipocytes more closely resemble pre-adipocytes and this is associated with 
increases in pro-inflammatory cytokine production [30]. Whereas, tPVAT a more brown-like phenotype, has less inflammatory cytokines and has higher expression of uncoupling protein-1. In addition to adipocytes, PVAT consists of adipose precursors and resident immune cells. In healthy depots of adipose tissue alternatively activated macrophages [31, 32], B-1 cells [33], innate lymphoid cells [34], and eosinophils [35-37] release anti-inflammatory cytokines, support adipose tissue function, and in PVAT promote beneficial adipose-vascular interactions $[33,35]$. In combined, the secretory profile of the tPVAT environment can influence the structure and function of the aorta through paracrine signaling and local circulation [38].

\section{Function of Perivascular Adipose Tissue in Health}

In health, the tPVAT is catabolic breaking down nutrients and fueling the electron transport chain. However, high expression of UCP-1 inhibits ROS production [39] and redirects the electron flux to produce heat instead of ATP production. The production of heat from the tPVAT is thought to be an important thermoregulatory mechanism [23]. UCP-1 expression and heat production are the hallmark of the brown-like tPVAT phenotype. The brown-like adipose tissue phenotype, in part, is supported through NO signaling. The importance of NO signaling for mitochondrial biogenesis and the brown adipose phenotype is highlighted in studies utilizing eNOS null mice $[40,41]$. In addition to the production of heat, in 1991 the observation had been made that PVAT blunted the contractile response of the aorta, but was dismissed as merely agonist uptake [42]. It would be 11 years later tPVAT derived cytokines regulation were first uncovered in 2002 [43]. Löhn and colleagues [43] exposed aortas with and without tPVAT to increase doses of multiple vaso-constrictors and collected the bath solution from aorta with 
tPVAT and added it to the bath of aortas without tPVAT. They showed the anti-contractile effects could be transferred. These experiments clearly demonstrate anti-contractile actions of tPVAT were not due to agonists uptake. Finally, the study showed preheating the transfer solution abolished the transfer of the anti-contractile effects, which led to the conclusion tPVAT releases vasoactive peptide(s), which blunt the aortic contractile response. This crucial experiment opened the door for subsequent evaluations of tPVAT function and coined the term PVAT derived relaxing factor (PVRF). The direct influence of tPVAT on smooth muscle cell relaxation is under intensive study. Numerous PVFRs have been proposed including: hydrogen sulfide, NO, palmitic acid methyl ester, adiponectin, and angiotensin (Ang) 1-7, and are highlighted in a recent review on the topic [44]. Five years after the discovery of an unknown PVRF, Gao et. al. [45] showed that PVAT could activate endothelial NO production, laying the ground work for PVAT regulation of the endothelium.

\section{Perivascular Adipose Tissue Autocrine and Paracrine Mediators}

Adiponectin may be the most prominent adipokine in healthy tPVAT. Through autocrine signaling adiponectin is a potential mediator of the brown-like phenotype through NO signaling [46]. Adiponectin is the prominent anti-inflammatory adipocyte acting to promote alternative polarization of macrophages and preventing the classic polarization $[47,48]$ in PVAT. These actions on resident macrophages help to prevent infiltration by pro-inflammatory immune cells into the adipose tissue due to lower production of chemoattractants [49]. Further evidence identifies adiponectin regulation of interleukin (IL)-10 as an important anti-inflammatory signal in macrophages [50]. IL-10 functions to reduce ROS and inhibit pro-inflammatory cytokine production [51, 52]. Although its potential role as PVRF has been dispelled [53], adiponectin 
released from PVAT mediates endothelial function via NO production [54]. Specifically, adiponectin regulates NO bioavailability by increasing transcription and activation of eNOS [5558]. Additionally, IL-10 release from healthy tPVAT may act to suppress ROS production and promote eNOS expression in the aortic endothelium [59, 60]. As discussed above the promotion of NO signaling promotes compliance of the aorta. Finally, PVAT is known to express the full complement of angiotensin system [61]. Expression of the angiotensin converting enzyme 2 (ACE2) is of interest as it is responsible for the breakdown of the vaso-constrictor Ang2 yielding Ang 1-7 [62], which is shown to cause vasodilation through actions on eNOS [63]. With tPVAT signaling supporting endothelium NO, any changes to tPVAT function may alter this beneficial relationship. Indeed, multiple disease states induce change is PVAT phenotype and function.

\section{Role of Perivascular Adipose Tissue in Vascular Pathologies}

\section{Metabolic Syndrome}

The prevalence of the metabolic syndrome (MetS) in the United States is 34\% [64] and the CDC estimated medical costs for MetS is over $\$ 300$ billion annually. MetS is collection of risk factors including dyslipidemia, obesity, hypertension, and elevated glucose, which elevates risk of diabetes, cardiovascular disease, and cardiac events [65]. Pathologically, MetS presents with increases in pro-inflammatory cytokines and oxidative stress [66-68]. Circulating elevations of pro-inflammatory cytokine are attributed to expansion and dysfunction of the visceral adipose tissue. Consequently, this is associated with large arteries in MetS suffer from reduced NO bioavailability and undergo ECM remodeling causing increases in arterial stiffness [69]. Elevation of aortic stiffness in MetS is one mechanism driving the increasing risk of cardiac events. Aortic stiffness regardless of disease status is an independent risk factor for CVD and 
cardiac events $[20,21]$. This association arises from the direct impact of stiffness on the heart, increasing afterload and reducing coronary flow [4]. Aortic stiffness in MetS arises from loss/ fragmentation of elastin fibers, deposition of collagen, collagen cross linkage, and smooth muscle tone. While aortic stiffness may be exacerbated by hypertension [70] in MetS, evidence suggests aortic stiffness may precede the development of hypertension [71, 72]. The chronic ROS production in MetS not only quenches NO but also causes fragmentation of elastin fibers R. Additionally, MetS is associated with increases of matrix remodeling protein expression specifically, MMP-9 and MMP-2 R. Both MMP-9 and MMP-2 may alter aortic compliance through their actions as elastases. Complicating the fragmentation of elastin is the increased deposition of type 1 collagen. Collagen type 1 is more rigid than that of collagen type 3 and elevation of expression throughout the vascular wall increases aortic stiffness [73, 74]. On top of reduction of elastin and increase collagen, glucose and ROS elevation of advanced glycation end product cause cross-linkage of ECM fibers further increasing aortic stiffness [75].

\section{Perivascular Adipose Tissue in Metabolic Syndrome}

Similar to the white adipose depots in MetS, it appears thoracic and peripheral PVAT adipocytes increase in size $[54,76]$. This coincides with increases in ROS production and inflammatory cytokines. Work in the New Zealand obese (NZO) mice model of MetS shows increased expression of cell adhesion molecules in tPVAT [76]. Cell adhesion molecules assist in immune cell infiltration of tPVAT, which may perpetuate the pro-oxidative/inflammatory environment. In accordance with this, PVAT in NZO mice showed higher levels of ROS production, in part, due to increased NOX activity and decreased expression of all 3 SOD isoforms [76]. However, the contribution to total ROS and the specific isoforms of NADPH oxidases involved need further 
evaluation. Furthermore, there appears to be a differential effect of MetS on NADPH oxidase ROS when comparing tPVAT to mesenteric PVAT in the NZO mouse. As such, the impact of NADPH oxidase ROS on subsequent regulation of aortic function warrants further investigation.

To date, the role of MetS tPVAT regulation of aortic function is unknown. Existing data examining how MetS affects adipose tissue and its role on aortic function comes from either, other PVAT depots, from animal models of MetS components, or from other vascular beds (mesenteric or small skeletal muscle arteries). The role of the MetS on tPVAT and in turn, the role of tPVAT on aortic function is likely dependent on the complex interactions of its components, which may yield differing effects than a component in isolation. I have described below some of the main findings from the auxiliary studies. Uncovering the distinct and coordinating signaling pathways of the MetS components in PVAT should be the topic of future evaluation. The two most common components of MetS studied in PVAT are obesity and hypertension

\section{Perivascular Adipose Tissue in Obesity}

Obesity results in expansion of adipocyte mass, tissue volume, and whitening of tPVAT in obesity [54, 76-78]. Hypertrophy of PVAT adipocytes is complicated by a reduced micro-vessel network leading to regional hypoxia [79-81]. Hypoxia is a potent signal increasing proinflammatory cytokine production and chemo-attractants. Another hallmark of beige adipose or PVAT during obesity is a shift toward whiter phenotypes. It is speculated during the development of obesity during tissue expansion that PVAT undergoes some degree of dedifferentiation and whitening, which results in upregulation of pro-inflammatory cytokines and 
immune-attractant chemokines [30]. However, the role of regional hypoxia or the direct initiation of the dedifferentiation is poorly understood. Persistent hypoxia may lead to adipocyte death, a hallmark of obese adipose depots, which attracts macrophages and up-regulates TNF $\alpha$ production [82]. Resident PVAT macrophages are predominantly M2 polarized in health aiding in promote of an anti-inflammatory environment $[83,84]$. Upon the onset of obesity, it is likely these macrophages shift to a pro-inflammatory phenotype and signal to attract T-cells and monocytes to the PVAT $[84,85]$, leading to accumulation of PVAT pro-inflammatory immune cells $[37,86]$. Macrophage infiltration appears to be essential in obesity induced PVAT dysfunction [86]. Upregulation of inflammatory cytokines promote further signaling for cell adhesion and chemokine production [87-89]. In addition, 2.5 hours of hypoxic conditions in tPVAT abolished the anti-contractile effect, which was restored with an anti-TNF $\alpha$ antibody. This suggests hypoxia induces TNF $\alpha$ expression and release from hypoxic tPVAT [54]. This is supported by experiments in PVAT and other adipose depots displaying increased TNF $\alpha$ and MCP-1 $[77,90]$. Hypoxia may additionally enhance the inflammatory burden of elevated fatty acids mediated through increased ROS production [91]. Obesity rapidly induces alterations in fatty acid composition directly leading to PVAT and adipose dysfunction [92, 93]. Another study however, suggests PVAT undergoes compensatory measures to retain the brown-like phenotype proceeding PVAT dysfunction and whitening in early obesity [94]. In this study Gil-Ortega et. al. [94] shows increases in NO in early obesity, which promotes the brown-like phenotype. However, this is eventually lost once inflammatory cytokine production and ROS increases [9597]. 
There is opposing evidence in tPVAT, which suggests it is protected against the pathophysiology of obesity [22]. Fitzgibbons et. al. [22] described a protection from diet induced obesity related infiltration of pro-inflammatory macrophages. This coincides with retention of UCP-1 expression and the brown phenotype and no increase in pro-inflammatory cytokine production after 13 weeks and 20 weeks of high fat diet. However, these experiments were conducted on tPVAT surrounding the aortic arch and might represent further regional differences in tPVAT between the arch and descending thoracic. Additionally, longer durations may be needed to initiate detrimental signaling in tPVAT as 8 months of high fat diet in the same mouse strain shows tPVAT inflammation. While we have some general understanding of obesity mediated changes in tPVAT, much is still left to be cleared up such as:

- What are the initiation signaling events in early obesity?

- Acute effects of caloric excess?

- What role does PVAT-adventitial fibroblast signaling play?

- What is the time course of immune cell infiltration?

- What role do resident immune population play?

- Do the different PVAT depots respond the same and over the same time course?

- Additionally, we need a well-defined tPVAT profile in health to understand the changes associated with obesity?

- How are these effected by concomitant components of MetS? 


\section{Figure 1.2. Image of obesity induced PVAT dysfunction}
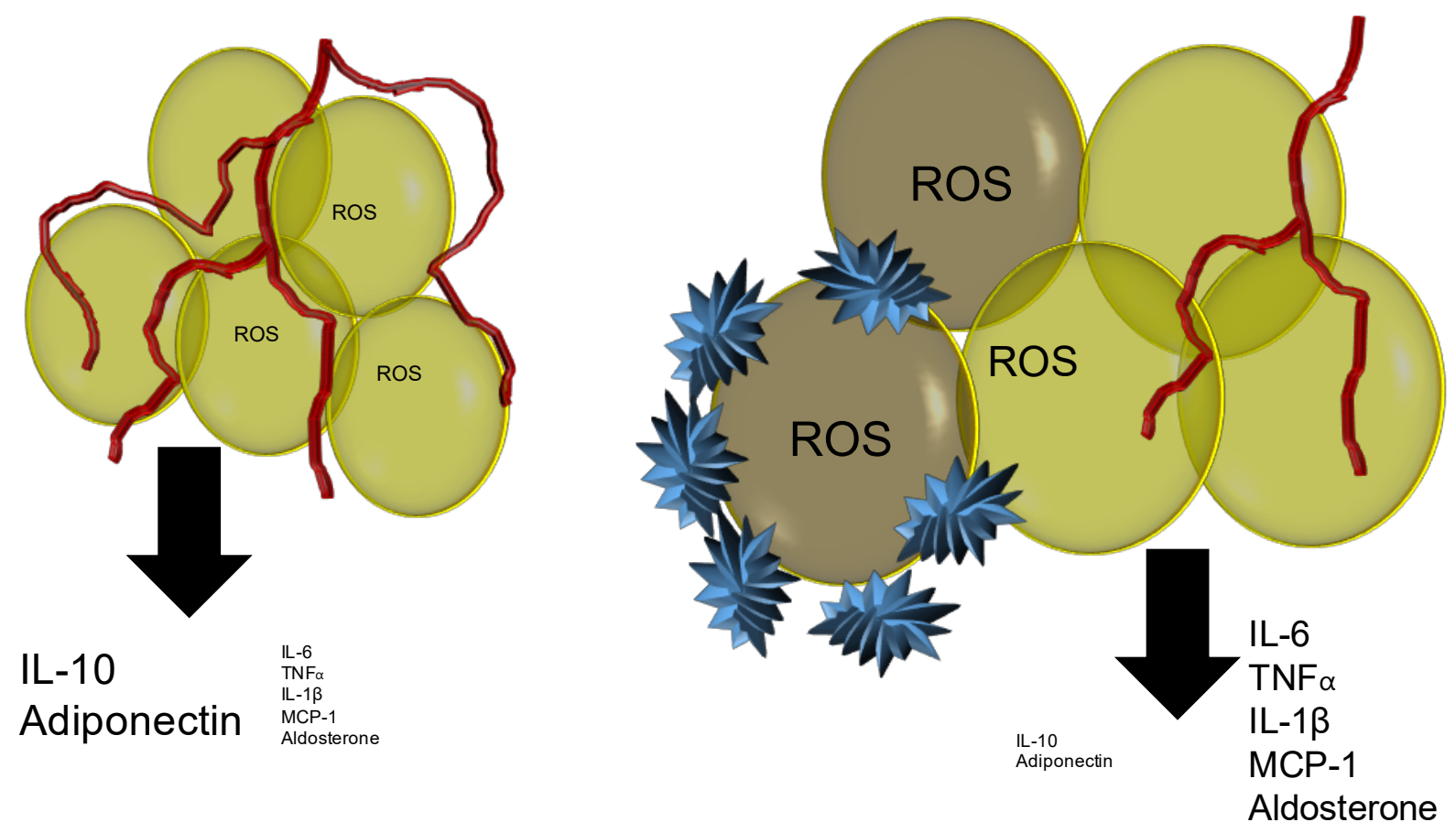

Oxidative Environment of Perivascular Adipose Tissue in Obesity

Alterations in PVAT phenotype (whitening), Immune cell infiltration (T-cells \& macrophages), hypoxia, and fatty acids contribute to oxidative stress in PVAT [76, 91-93, 98-101] (Fig.1.2). ROS causes oxidative damage to cellular components as well as sequesters NO, drives phenotypic whitening, which can further exacerbate ROS production [39]. Oxidative stress in PVAT appears to be related to both increasing ROS production and loss of the antioxidant system. The shift toward the pro-oxidative state develops early as 3 weeks of fructose feeding induced obesity reduced the superoxide dismutase and glutathione defense systems by half resulting in a doubling of oxidative stress markers [93]. The infiltration of immune cell brings higher expression of oxidative enzymes, chiefly NOX [102], into the PVAT. Numerous studies 
in various PVAT depots support the idea of NOX as an important mediator of ROS in obesity. In the abdominal aorta gene expression of NOX subunits were increased along with PVAT ROS generation. The elevated ROS could be abolished with the general NOX inhibitor apocynin [93]. In mesenteric PVAT an expression of NOX subunit $\mathrm{p}^{67 \mathrm{phox}}$ has been recorded [100] and elevated NOX activity coinciding with increased PVAT ROS [76, 97]. Similarly, tPVAT in obese mice show elevated ROS production and greater than 2-fold increased gene expression for numerous NOX subunits [99]. Contrary, to these findings of obesity induced NOX ROS production, Sanchez et al. [103] suggest long term high fat diets decrease expression of NOX subunits in mesenteric PVAT. These results are hard to interpret in the context of the greater literature as high fat diets were implemented after weaning for 5 months, longer than other diet induced obesity protocols, however the diet did not induce weight gain compared to the controls despite a $100 \%$ increase in chow calorie content [103]. While these collective works make a strong case for NOX in obesity induction of PVAT ROS, other mechanisms have been reported. One such study showed tPVAT uncoupled eNOS was solely responsible for increased ROS with obesity. eNOS uncoupling occurs without cofactors important for dimerization or substrate depletion, in this active monomeric state eNOS reduces atmospheric $\mathrm{O}_{2}$ to superoxide [104]. Xia et. al. [101] showed superoxide production was 6 times higher in obese tPVAT, which was abolished when tPVAT was pretreated with a NOS inhibitor (L-NAME). The uncoupling in obese tPVAT was due to diminished eNOS substrate L-arginine caused by the increased activity of the alternate arginase pathway [101]. Immune infiltration and NOXs were not evaluated in the study. ROS in itself can cause eNOS uncoupling through oxidation of tetrahydrobiopterin $\left(\mathrm{BH}_{4}\right)$. It is likely in the studies reporting increase ROS and increased expression or activity of NOX, PVAT undergoes some degree of uncoupling. With the overwhelming evidence for immune cell 
infiltration and increases in NOX activity, it is highly unlikely uncoupled eNOS is the only enzyme producing ROS. Alternatively, it may be L-NAME treatment through inhibition of immune cell iNOS decreased the stimulation of NOX ROS production [105]. Another alternative source of PVAT ROS comes from recent evidence suggesting mitochondrial ROS production is an essential mechanism of PVAT dysfunction, depicted in a study by da Costa et al. [98], which showed an account of mitochondria function in obese tPVAT. The authors suggested no change in TPVAT mitochondria number as a result of obesity. However, the mitochondria in obese tPVAT expressed less UCP-1, consumed less $\mathrm{O}_{2}$, and produced more ROS. It is clear many oxidative pathways may play a part in obesity and potentially MetS related PVAT ROS. Further investigations into the origin, time course, and relative contribution of various oxidative enzyme to tPVAT ROS production are merited.

\section{Perivascular Adipose Tissue ROS Impact on Vascular Function}

Superoxide is a short lived volatile radical, which likely doesn't diffuse to the endothelium from PVAT. However, the reaction of superoxide and water mediated by SOD produces the more stable hydrogen peroxide $\left(\mathrm{H}_{2} \mathrm{O}_{2}\right) . \mathrm{H}_{2} \mathrm{O}_{2}$ production was shown to be produced by PVAT and to induce vascular relaxation independent of the endothelium [45]. In obesity, the $\mathrm{H}_{2} \mathrm{O}_{2}$ production is elevated despite the loss of SOD levels. Pre-treatment of abdominal aorta PVAT with PEGcatalase (the enzyme which breaks $\mathrm{H}_{2} \mathrm{O}_{2}$ into $\mathrm{H}_{2} \mathrm{O}$ and $\mathrm{O}_{2}$ ) prior to aortic EDD had differing effects dependent upon obesity status. In lean PVAT $\mathrm{H}_{2} \mathrm{O}_{2}$ scavenging causes impaired EDD of the abdominal aorta, while in obese PVAT EDD was improved. This suggests there is an ideal $\mathrm{H}_{2} \mathrm{O}_{2}$ concentration window and once $\mathrm{H}_{2} \mathrm{O}_{2}$ is elevated with obesity it causes vascular impairment. Additionally, ROS may directly cause fragmentation of elastin fibers in the aorta 
[106]. While no experiment has directly tested the impact of obesity induced PVAT ROS on vascular stiffening, studies in another oxidative disease such as aging implicate a link. Fleenor et al. [107] showed treatment of aging tPVAT with the free radical scavenger TEMPOL inhibits PVAT mediated stiffening of the aorta.

\section{Perivascular Adipose Tissue Proteasome Function}

The oxidative load may also impair the ubiquitin-proteasome system (UPS) in PVAT. Currently, there is no direct indication of this, but evidence from other tissue can be used to draw speculation. Recently, both obese human and mouse visceral adipose tissue showed reduced proteasome activity and was linked to development of insulin resistance, a hallmark of MetS [108]. We speculate the importance of proteasome function is magnified in the protein dense PVAT. Eukaryotic cells express the $26 \mathrm{~S}$ proteasome, which is comprised of the $20 \mathrm{~S}$ core bound to one or two 19S cap regulatory particles. The 19S cap facilitates substrate [109] recognition and 20S gate opening [110] feeding ubiquitinated and damaged proteins into the 20S core [111]. It has been speculated higher oxidative environments may cause the 19S cap to dissociate from the $20 \mathrm{~S}$ core reducing the ability to recognize and degrade ubiquitin [112]. This may potential cause the buildup of proteasome substrates, as ROS is known to damage and cause misfolding of proteins [113]. The increase accumulation of damaged and misfolded proteins can lead to cellular and oxidative stress [114-116]. Specifically, buildup of oxidized and ubiquitin through activation of endoplasmic reticulum stress induced production of inflammatory cytokines [115]. Taken together this collection of literature may suggest PVAT proteasome function may modulate inflammatory cytokine production and participate in MetS induced PVAT dysfunction. 
Future endeavors are needed to assess the causative and/ or exacerbating role of the entire UPS in PVAT dysfunction.

\section{Inflammation of Perivascular Adipose Tissue in Obesity}

Ubiquitin $[115,116]$ and ROS [117] are two known stimulators of inflammatory cytokines, which are indeed increased in obese PVAT. TNF $\alpha$ is probably the most studied cytokine associated with PVAT. In obesity gene expression and PVAT concentration greatly increase. TNF $\alpha$ has both autocrine effect on PVAT and paracrine effect on the underlying vessel. TNF $\alpha$ activates the production of ROS from oxidative enzymes, such as NADPH oxidase [118, 119], giving it the ability to affect both PVAT and vascular ROS. Infiltrating macrophages in obesity play a major role in adipose production of TNF $\alpha$ partially in response to tissue hypoxia [90, 120]. Greenstein et al. [54] showed TNF $\alpha$ can be induced even in healthy PVAT, presumably from repolarize of the resident macrophages and adipocytes, as macrophage infiltration should be low. In this study TNF $\alpha$ exposure in healthy rat mesenteric arteries with intact PVAT repealed the anti-contractile effect of PVAT. However, the role of PVAT is confounded because TNF $\alpha$ was acting on both PVAT and the vessel. Assessment of small gluteal arteries showed higher expression of TNF receptors in the PVAT of MetS patients with loss of anti-contractile effects. This however, could not be reversed by TNF receptor blockade. More data in visceral small arteries from Virdis et al. [121] shows increased PVAT TNF gene expression and increase TNF receptor in PVAT and the vascular wall of obese patients. TNF $\alpha$ stimulation of NADPH oxidase ROS was found in the vessel wall causing reduced NO, which was reversed with TNF $\alpha$ neutralization. However, immunofluorescent detection of TNF $\alpha$ was strongest in the vessel wall. Due to experimental design, it is hard to determine the exact impact of TNF $\alpha$ produced in the 
PVAT compared to that of vascular origin. The acute effects of TNF $\alpha$ are likely mediated by this activation of vascular NOX, while chronic effects further impair vascular function through inhibition of eNOS, which may link PVAT dysfunction with pathological vascular changes such as remodeling and stiffness. In addition to activation of ROS TNF $\alpha$ also controls other cytokine expression through direct and indirect pathways [122]. It is clear TNF $\alpha$ plays a major role in obese PVAT dysfunction. However, much of what we know comes from TNF receptor inhibition in small artery with intact PVAT. Further research is needed in conduit artery PVAT and to determine signaling pathways mediating TNF $\alpha$ actions in PVAT and the vascular wall.

Adiponectin \& Interleukin 10. In the context of PVAT one of the most important targets effected by TNF $\alpha$ is adiponectin. As discussed above adiponectin plays a number of important roles in mediating healthy PVAT signaling. Increased TNF $\alpha$ inhibits adiponectin removing its effects on PVAT and vascular NO [57]. This leads to perpetuation of the whitening phenotype of PVAT and loss of PVAT assistance in EDD. Obesity also results in the loss of anti-TNF $\alpha$ cytokines like IL-10. IL-10 in produced primarily in M2 polarized macrophages and adipocytes so as the various obesity induced mediators drive repolarization expression and release of IL-10 drops [32, 123]. This is important because IL-10 inhibits NOX enzymes, which is a major source of both vascular and PVAT ROS in obesity [124]. Additionally, IL-10 inhibition of TNF $\alpha$ actions is lost in obesity exacerbating its increase [59].

Leptin. Leptin is another important anti-inflammatory PVAT cytokine, which can induce vasorelaxation through multiple pathways [125]. However, in obesity leptin levels increase as 
resistance to leptin signaling develops, much like insulin resistance, this may exacerbate vascular impairment [126].

Interleukin 1 beta. TNF $\alpha$ mediates expression of IL-1 $\beta$ [127], another stimulator of oxidative enzymes [128]. Additionally, IL-1 $\beta$ can act to enhance TNF $\alpha$ signaling through regulation of TNF receptors [129]. This implicates IL-1 $\beta$ in a supportive role to TNF $\alpha$ in mediating obese regulation of PVAT/ vascular dysfunction.

Thrombospondin 1. Another action of TNF $\alpha$ is upregulation of thrombospondin-1 (TSP-1) [130]. TSP-1 is expressed in adipocytes however; it has not been evaluated in the context of PVAT pathophysiology. TSP-1 has been shown to inhibit eNOS function and impair EDD [131, 132], thus it may participate in both phenotypic whitening and vascular dysfunction. TSP-1 may further perpetuate in adipose dysfunction through its anti-angiogenic actions, potentially participating in PVAT hypoxia and immune cell infiltration $[133,134]$.

Matrix Metalloproteinase 9. TNFa may also meditate ECM remodeling and stiffness through actions on PVAT MMPs. Both adipocytes and immune cells express MMPs [135, 136], in particular, MMP9 which is highly associated with aortic stiffness and displays elastase activity [137]. The fragmentation of elastin increases aortic stiffness by causing the loading of collagen fibers at lower pressures [4]. First, TNF $\alpha$ can stimulate the production of MMP9 directly [138]. Second, TNF $\alpha$ can indirectly stimulate MMP9 though its promotion of other cytokines, whereby both IL-1 $\beta$ and TSP-1 can activate MMP9 [139, 140]. Active MMP9 can feedback to cleave TNF $\alpha$ [141] and IL-1 $\beta$ into active forms [142] possibly creating a cycle of upregulation between 
remodeling proteins and pro-inflammatory cytokines. One study has shown increased MMP9 protein levels in aortic tissue, which may have included PVAT, as "aortic tissue" used for imaging clearly contains PVAT [99]. Expression of MMPs in PVAT and their actions on the elasticity of the underlying vessel are important mechanisms to understand PVAT-vessel interactions.

Interleukin 6. IL-6 is another well studied PVAT cytokine in obesity. It appears the major role of IL-6 is autocrine signaling directing immune infiltration [143] and T-cell pro-inflammatory polarization [144]. IL-6 exposure on health small artery with PVAT impaired function and IL-6 blockade restored anti-contractile properties in hypoxia exposed vessels [54]. This may be due to actions of IL-6 activation of endothelium ROS and inhibition of eNOS [145, 146], however this has not been shown directly in obese PVAT. The role of PVAT IL-6 in obesity induced aortic stiffness is unknown, but evidence from aging suggests it may contribute [147].

Aldosterone. Aldosterone, similar to TNF $\alpha$, can activate ROS production through activation of NOX enzymes [148]. Recently the angiotensin-aldosterone system has been identified in PVAT/ adipose tissue [149]. Expression of aldosterone was shown to be increased in obesity and inhibition of aldosterone receptors in mesenteric arteries with intact PVAT blunted the PVAT induced impairment of EDD [150]. This suggests a role of the local RAAS system in obesity mediation of vascular impairment however this has not been shown in PVAT and the aorta. New evidence suggests the impairment of vascular function by aldosterone is mediated by angiotensin receptors and not mineralcorticoid receptors [151] making the assessment of aldosterone vascular effects more difficult. While a lot of work has been done to show the impact of 
individual mediators of autocrine regulation of PVAT dysfunction and paracrine vascular dysfunction much more work is needed to interpret how all of these mediators work in concert or opposition of one another, a representative diagram is shown in figure 1.3.

Figure 1.3. Depiction of known and potential PVAT regulation of vascular function.

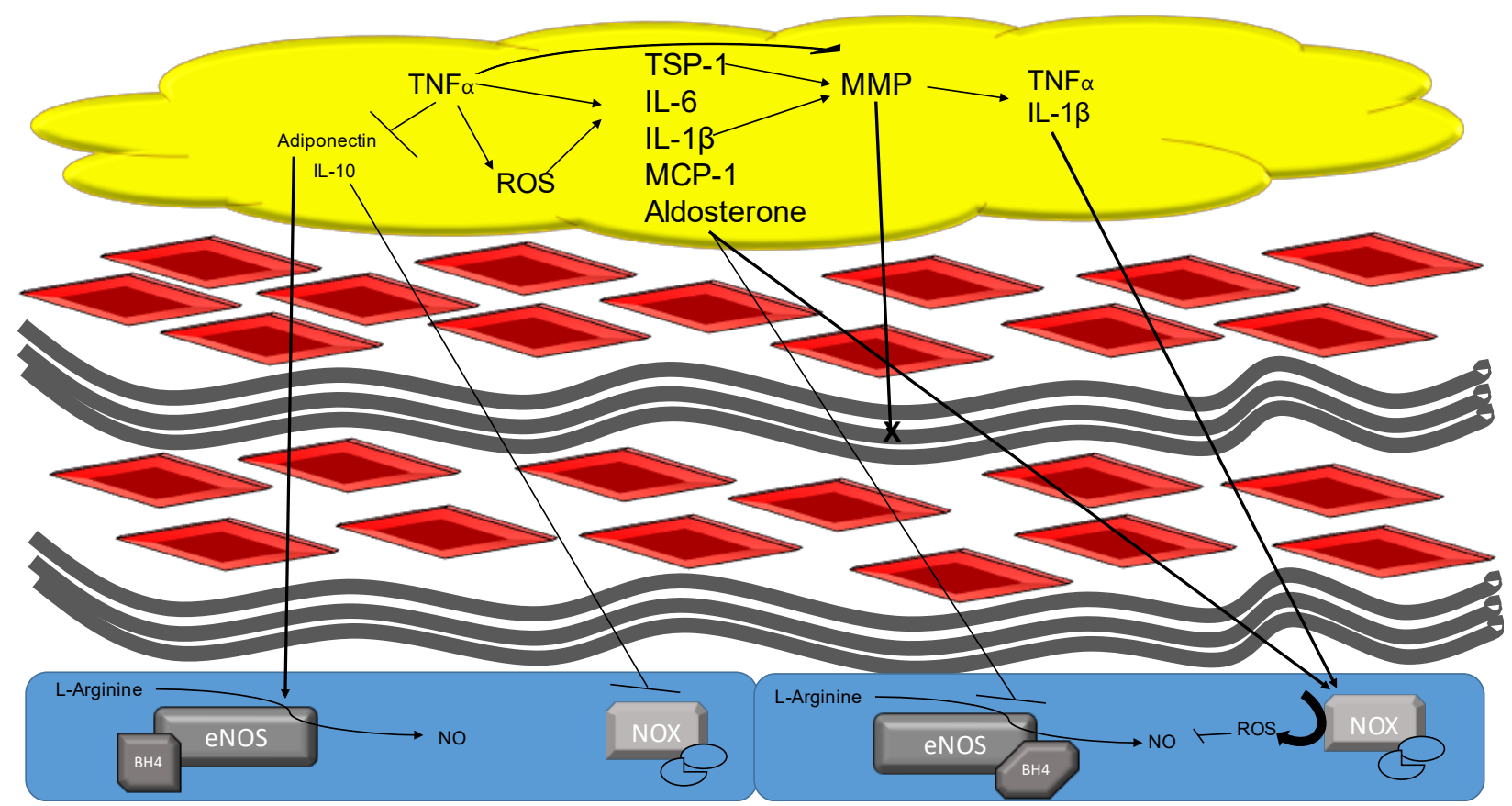

\section{Impact of Hypertension on Perivascular Adipose Tissue}

The effect of hypertension on PVAT has been less well studied than obesity however the Harrison lab has performed a few well-designed studies to implicate T-cells in PVAT hypertension pathology. They first made the observation vascular function was preserved in Ang II induced hypertensive mice, which were T-cell null. Immunohistochemical evaluation of the aorta showed the T-cells preferentially infiltrated the tPVAT [152]. This study was followed up by an in-depth characterization of tPVAT immune cell population and the chemotactic cytokines involved [153]. The study concluded a very specific subset of T-cells was drawn to the PVAT by 
CCL5 production in the tPVAT. This subpopulation constituted a sizable portion of the infiltrating cells. Blocking CCL5 reduced ROS production and T-cell infiltration of tPVAT. Importantly, this class of T-cells expressed high levels of interferon gamma (IFN- $\gamma$ ) and IL-17, which they showed IFN- $\gamma$ drastically reduced EDD after an extended incubation. Infiltrated immune cell release of IL-17 may play an important role in recruitment of other immune cells [154]. The only drawback from the study is IFN- $\gamma$ production was not quantified from tPVAT and an arbitrary concentration of IFN- $\gamma$ was used in EDD experiments. However, this is supported by work from another group showing IFN- $\gamma$ expressing immune cells regulate vascular impairment in hypertension [155]. These works present ample evidence for PVAT pathology being mediated through this subpopulation of high IFN- $\gamma$ expressing T-cells, which relies on adipocyte and resident immune cells production of CCL5. Previous work had established loss of anti-contractile effects of PVAT in spontaneously hypertensive rats [153]. However, this was not due to IFN- $\gamma$ but rather the loss of the NO promoting peptide Ang 1-7. Another proposed mechanism for PVAT mediated vascular dysfunction is decreased leptin expression in hypertension [156]. These two models may however not represent the pathological development of hypertension in MetS, which may confer different effects on PVAT function and aortic regulation, especially concomitantly with other components of MetS. Additionally, these studies highlight immune infiltration and ROS production as similarities between obesity and hypertension induced PVAT dysfunction. However, it appears different immune populations are implicated in the two disease states. Another similarity may be the activation of NOX enzyme ROS production. Some evidence suggests the IL-17 producing T-cells infiltrating PVAT result in activation of NOX enzyme ROS production $[157,158]$. It is suggested this is driven by IL-6 expression and release [144]. Understanding the IL-6 - IL-17 axis in PVAT MetS needs further 
exploration as IL-6 elevation is a common finding in obese PVAT. More studies are needed to understand the full effect of hypertension of PVAT and concomitantly with obesity or with other components of MetS.

\section{Chronic Stress and Depression States}

\section{Prevalence and Pathology}

Depressive psychological disorders are a common worldwide affliction. According to the World Health Organization, depressive states affect approximately 350 million people making it the leading global cause of disability. The burden of depressive states is at its highest in the USA with prevalence rate of roughly $17 \%$ [159]. The complex psycho-physiological interactions of depressive states cause numerous health issues including CVD [160, 161]. Clinical evaluations of arterial stiffness [162], a CVD risk factor [163], and flow mediated dilation [164] link vascular impairment with depressive symptoms. The link between depressive states and vascular function have slowly come to light in recent history; a detailed account of the advancements in the field can be found in a review by Golbidi et. al [165]. In addition, there is a high coprevalence of depressive states with obesity/ metabolic syndrome (MetS) [166, 167] and little is known about the physiologic consequences of this comorbidity. With one study showing worse behavioral outcomes in the comorbid state [168]. Despite the growing evidence in the vasculopathy of depressive states, the effect on tPVAT regulation of aortic function is unknown.

\section{Animal Model of Depressive States}


The unpredictable chronic mild stress (UCMS) protocol is a well-defined model to induce a depressive state in rodents $[169,170]$. Rodents undergoing UCMS manifest with clinically relevant depressive symptoms such as anhedonia, disordered sleeping patterns and learned helplessness [169-172]. A review of UCMS reveals alteration in brain structure and function, parallel to clinical depression [173] and importantly, the behavioral outcomes used to validate depressive states can be reversed by treatment with antidepressants further validating the use of UCMS to study depressive states in rodents [174].

\section{Effect of Chronic Stress on Physiological Function}

Exposure to chronic stress activates two major pathways: hypothalamus-pituitary-adrenal (HPA) axis and the sympathetic nervous system.

\section{Vascular Effects}

The Frisbee lab has conducted one of the only studies to evaluate the impact of depressive states on conduit vessel function [175]. In this study 8 weeks of UCMS reduced aortic EDD in mice, due to reduced NO bioavailability. The endothelial NO independent component of relaxation was increased following UCMS due to the dilatory actions of $\mathrm{H}_{2} \mathrm{O}_{2}$. However, it was not enough to compensate for the loss of NO. Circulating factors did not correlate well with the observed impairment suggesting a possible outward in disease signaling.

\section{$\underline{\text { Glucocorticoids }}$}

Glucocorticoids are the end effector of the HPA axis, cortisol (in humans) or corticosterone (in rodents) and are shown to impair endothelial function. Cortisol and corticosterone are shown to 
reduce NO bioavailability $[176,177]$ and regulate a gene promoter of eNOS [178]. Indirectly, glucocorticoids activation of ROS production $[179,180]$ can interfere with NO or eNOS cofactors leading to eNOS uncoupling and perpetuation of the oxidative load [181, 182]. The increased $\mathrm{H}_{2} \mathrm{O}_{2}$ component of relaxation in the Frisbee study may due to corticosterone actions leading to increased SOD activity and decreased catalase activity [183]. However, this has only been described in the liver and many conflicting accounts of corticosterone effects on the antioxidant system have been reported, which may be further complicated by tissue specificity $[180,183,184]$. Uncovering the endothelial antioxidant response to corticosterone warrants further investigation. Importantly, these are effects of chronically elevated glucocorticoids as acute high dose of corticosterone was shown to activate eNOS through AKT [185]. The comorbidity of UCMS with high fat diet was shown to not cause any further impairment to eNOS expression, but at extended time points UMCS decreased the nitrate/nitrite levels below that of just the high fat diet suggesting an additional inhibition of NO through a nontranscriptional pathway [186]. Additionally, this study showed increased MMP9 expression in the aorta [186], suggesting the comorbidity may exacerbate the effects on aortic stiffness.

\section{Sympathetic Nervous System}

Sympathetic signaling in the blood vessels leads to vasoconstriction, in the context of the aorta smooth muscle contraction increases the loading of collagen fibers and stiffens. Highlighted by a recent study showing blockade of sympathetic input results in decreased aortic stiffness [187]. One of the major effects of increased SNS activity is activation of the RAAS, which contribute to CVD risk [188-190]. Ang II and aldosterone are the predominant vasoactive mediators of RAAS. Both can stimulate endothelial ROS production $[148,191,192]$. The increased ROS 
appears to be mediated by activation of NOX [193-195] and increased transcription of NOX components [194, 196, 197].

\section{Adipose Effects}

The effects of UCMS on PVAT function have never been evaluated. However, we can speculate based on the impact of UCMS on physiological function and other adipose depots. An interesting finding, which has implications for tPVAT, is the effect on brown adipose tissue. Starck et al. [198] report corticosterone reduced UCP-1 expression and heat generation while lipid storage increased. Suggesting elevation of corticosterone in UCMS may lead to phenotypic whitening in tPVAT. Additionally, UCMS contributes to altered lean/adipose ratio, macrophage infiltration, glucose metabolism, insulin resistance, and inflammation in adipose tissue [199, 200]. Glucocorticoids normally suppress immune function and pro-inflammatory cytokines [201, 202]. However, chronic exposure leads to glucocorticoid resistance and upregulation of inflammatory cytokines $[203,204]$. This is especially troublesome for adipocytes and resident macrophages as studies showed glucocorticoid resistance, Ang II and SNS activation all increase adipose cytokine produce (i.e. TNF $\alpha$ ) [205-209]. The increased production of inflammatory cytokines has detrimental impact on both the adipose tissue and vasculature as discussed above. As chronic stress activates the systemic RAAS it may as well activate local RAAS. Adipose and PVAT are shown to expression local RAAS and as discussed previously enzymatic activity of ACE2 is important in breaking down Ang II to produce Ang 1-7 [62] a PVAT derived vaso-relaxant. However, the impact of UCMS on local RAAS is unknown. Signaling of Ang II may alter adipose blood flow distribution, increase SNS activity, increase oxidative stress and promote lipogenesis $[61,210]$ contributing to adipose tissue dysfunction. While evidence suggests UCMS 
may initiate PVAT dysfunction, extensive investigations into the effect of UCMS and depressive states on PVAT are needed. Furthermore, many of these signaling pathways bare resemblance to obesity related vascular and adipose dysfunction. Determining the activation and consequences of these pathways in the comorbid state of obesity with depressive like states warrants investigation.

\section{Physiological Changes Exerted by Exercise}

Aerobic exercise is a common therapy used in treating both MetS and depressive states as both disease states normally present with diminished physical activity [211]. It is well known aerobic exercise training (Ex) reduces the risk of cardiovascular disease, events, and mortality [212, 213] and improves vascular function in these disease states $[214,215]$. This is partially due to the restoration of NO bioavailability, reduced stiffness, and restoration of oxidant/ inflammatory mediators. Ex has also long been recognized for its anti-depressant effects [216]. The major mechanism regulating vascular and adipose function is the reduction of SNS activity following Ex [217]. The beneficial effects of Ex on vascular and adipocyte function are discussed below.

\section{Vasculature Effects}

Ex improves NO bioavailability multiple pathways. Ex is shown to increase eNOS gene transcription and protein levels $[218,219]$. This may be mediated through Ex induced $\mathrm{H} 2 \mathrm{O} 2$ production [220, 221]. Additionally, eNOS activation increased NO production and Ex increase both Akt and AMPK phosphorylation of eNOS [222]. Available levels of essential eNOS cofactors may also increase with Ex [223, 224] further augmenting NO. As discussed previously 
NO is an important regulator of aortic stiffness. Indeed, Ex has been shown to reduce aortic stiffness in disease states $[67,225,226]$. Accompanying the improved endothelial function, Ex both increases antioxidant defense and reduces oxidative enzyme ROS production [227, 228], which contributes to the increase bioavailability of NO and eNOS cofactors. Ex also decreases the levels of pro-inflammatory cytokines [229]. Reduced vascular wall inflammation starts with shear stress mediated reduction of cell adhesion expression [230, 231]. However, the main antiinflammatory effect of Ex is through its effects on the adipose tissue.

\section{Adipose Tissue Effects}

Studies show Ex promotes adipose NO signaling [40], which promotes mitochondrial biogenesis and phenotypic browning $[40,41]$. In turn, the brown-like phenotype helps to reduce the oxidative burden in the adipocyte [39]. Additionally, Ex alters polarization of resident immune cells [232, 233], thus further reducing the oxidative and inflammatory burden. Numerous studies have assessed Ex effect on visceral adipose tissue showing reduced levels of TNF $\alpha$ and IL-1 $\beta$ while Ex increased anti-inflammatory cytokines like IL-10 [234, 235]. Some evidence suggests the anti-inflammatory effects of Ex are independent of weight loss and adiposity [236, 237]. One potential mechanism of recued inflammation is Ex restoration of adipocyte profusion through angiogenesis [238, 239]. Restoration of adipose capillary density likely decreases the hypoxic stimulus for immune-attraction and inflammation. While direct evidence is lacking for exercise effect on adipose tissue aldosterone expression, indirect evidence shows aldosterone levels are correlated with body mass and weight loss [240-242]. To date only one study, has examined the effect of Ex on PVAT regulation of vascular function. In healthy rats, exercise had no impact on 
PVAT regulation of aortic function [243]. Further, studies are needed to access the therapeutic efficacy of Ex on tPVAT in disease state, like MetS.

\section{$\underline{\text { Summary and Purpose of the Dissertation }}$}

It is clear endothelial function of the aorta is important for maintaining healthy cardiovascular function. However, in disease states like MetS and depressive states reduced endothelial function and wall remodeling increase CVD risk. It is clear tPVAT regulates aortic function in health and plays a role in mediating vascular pathology in obesity and hypertension, but many gaps still exist in our knowledge of TPVAT function in MetS and its subsequent regulation of aortic endothelium and mechanical properties. TPVAT contribution to aortic dysfunction in depressive states is virtually unexplored and may play a part in vascular dysfunction based on observation from other adipose depots. However, the validity of these assumption need rigorous testing. It appears similar pathways (inflammation, RAAS, and SNS) may mediate the vascular pathology of depressive states as in MetS. Some studies suggest an additive effect of depressive states furthering tissue dysfunction in obesity in the comorbid state. The impact of the comorbidity in tPVAT is unknown and may bare import implications for understanding vascular function and CVD risk. Finally, Ex is known to reverse the detrimental pathophysiology of both MetS and depressive states. However, virtually nothing is known about Ex mediated effects in tPVAT.

For these reasons, we utilized a clinically translational model of MetS the obese Zucker rat and their lean counterparts for the purpose of filling in the gaps in our knowledge about pathways mediating tPVAT aortic function in MetS, depressive states, and Ex. This work has implications in understanding MetS and depressive disease progression. Additionally, we will determine the 
therapeutic efficacy of exercise in prevention of impairment of tPVAT and IPVAT regulation of aortic dysfunction. 
References:

1. Sokolis, D.P., H. Boudoulas, and P.E. Karayannacos, Segmental differences of aortic function and composition: clinical implications. Hellenic J Cardiol, 2008. 49(3): p. 14554.

2. Safar, M.E., et al., Pulse pressure, arterial stiffness, and end-organ damage. Curr Hypertens Rep, 2012. 14(4): p. 339-44.

3. Saji, N., et al., Association between silent brain infarct and arterial stiffness indicated by brachial-ankle pulse wave velocity. Intern Med, 2012. 51(9): p. 1003-8.

4. Lakatta, E.G. and D. Levy, Arterial and cardiac aging: major shareholders in cardiovascular disease enterprises: Part I: aging arteries: a "set up" for vascular disease. Circulation, 2003. 107(1): p. 139-46.

5. Sumpio, B.E., et al., Enhanced collagen production by smooth muscle cells during repetitive mechanical stretching. Arch Surg, 1988. 123(10): p. 1233-6.

6. Sutcliffe, M.C. and J.M. Davidson, Effect of static stretching on elastin production by porcine aortic smooth muscle cells. Matrix, 1990. 10(3): p. 148-53.

7. Galis, Z.S., et al., Cytokine-stimulated human vascular smooth muscle cells synthesize a complement of enzymes required for extracellular matrix digestion. Circ Res, 1994. 75(1): p. 181-9.

8. Langille, B.L., Arterial remodeling: relation to hemodynamics. Can J Physiol Pharmacol, 1996. 74(7): p. 834-41. 
9. Wilkinson, I.B., S.S. Franklin, and J.R. Cockcroft, Nitric oxide and the regulation of large artery stiffness: from physiology to pharmacology. Hypertension, 2004. 44(2): p. $112-6$.

10. Brandes, R.P., I. Fleming, and R. Busse, Endothelial aging. Cardiovasc Res, 2005. 66(2): p. 286-94.

11. Dao, H.H., et al., Evolution and modulation of age-related medial elastocalcinosis: impact on large artery stiffness and isolated systolic hypertension. Cardiovasc Res, 2005. 66(2): p. 307-17.

12. Fitch, R.M., et al., Nitric oxide synthase inhibition increases aortic stiffness measured by pulse wave velocity in rats. Cardiovasc Res, 2001. 51(2): p. 351-8.

13. Gurjar, M.V., R.V. Sharma, and R.C. Bhalla, eNOS gene transfer inhibits smooth muscle cell migration and MMP-2 and MMP-9 activity. Arterioscler Thromb Vasc Biol, 1999. 19(12): p. 2871-7.

14. Jenkins, G.M., et al., Increased expression of membrane-type matrix metalloproteinase and preferential localization of matrix metalloproteinase-2 to the neointima of ballooninjured rat carotid arteries. Circulation, 1998. 97(1): p. 82-90.

15. O'Rourke, M., Arterial stiffness, systolic blood pressure, and logical treatment of arterial hypertension. Hypertension, 1990. 15(4): p. 339-47.

16. Blomster, J.I., et al., Coronary flow reserve as a link between exercise capacity, cardiac systolic and diastolic function. Int J Cardiol, 2016. 217: p. 161-6.

17. Eroglu, S., et al., Association between coronary flow reserve and exercise capacity. Hellenic J Cardiol, 2015. 56(3): p. 201-7. 
18. Vlachopoulos, C., K. Aznaouridis, and C. Stefanadis, Prediction of cardiovascular events and all-cause mortality with arterial stiffness: a systematic review and meta-analysis. $\mathrm{J}$ Am Coll Cardiol, 2010. 55(13): p. 1318-27.

19. O'Rourke, M.F. and M.E. Safar, Relationship between aortic stiffening and microvascular disease in brain and kidney: cause and logic of therapy. Hypertension, 2005. 46(1): p. 200-4.

20. Laurent, S., et al., Aortic stiffness is an independent predictor of all-cause and cardiovascular mortality in hypertensive patients. Hypertension, 2001. 37(5): p. 1236-41.

21. Laurent, S., et al., Aortic stiffness is an independent predictor of fatal stroke in essential hypertension. Stroke, 2003. 34(5): p. 1203-6.

22. Fitzgibbons, T.P., et al., Similarity of mouse perivascular and brown adipose tissues and their resistance to diet-induced inflammation. Am J Physiol Heart Circ Physiol, 2011. 301(4): p. H1425-37.

23. Chang, L., et al., Loss of perivascular adipose tissue on peroxisome proliferatoractivated receptor-gamma deletion in smooth muscle cells impairs intravascular thermoregulation and enhances atherosclerosis. Circulation, 2012. 126(9): p. 1067-78.

24. Wahli, W., O. Braissant, and B. Desvergne, Peroxisome proliferator activated receptors: transcriptional regulators of adipogenesis, lipid metabolism and more. Chem Biol, 1995. 2(5): p. 261-6.

25. Harms, M. and P. Seale, Brown and beige fat: development, function and therapeutic potential. Nat Med, 2013. 19(10): p. 1252-63.

26. Berry, D.C., et al., The developmental origins of adipose tissue. Development, 2013. 140(19): p. 3939-49. 
27. Atit, R., et al., Beta-catenin activation is necessary and sufficient to specify the dorsal dermal fate in the mouse. Dev Biol, 2006. 296(1): p. 164-76.

28. Victorio, J.A., et al., Different Anti-Contractile Function and Nitric Oxide Production of Thoracic and Abdominal Perivascular Adipose Tissues. Front Physiol, 2016. 7: p. 295.

29. Galvez-Prieto, B., et al., Comparative expression analysis of the renin-angiotensin system components between white and brown perivascular adipose tissue. J Endocrinol, 2008. 197(1): p. 55-64.

30. Chatterjee, T.K., et al., Proinflammatory phenotype of perivascular adipocytes: influence of high-fat feeding. Circ Res, 2009. 104(4): p. 541-9.

31. Chawla, A., K.D. Nguyen, and Y.P. Goh, Macrophage-mediated inflammation in metabolic disease. Nat Rev Immunol, 2011. 11(11): p. 738-49.

32. Lumeng, C.N., J.L. Bodzin, and A.R. Saltiel, Obesity induces a phenotypic switch in adipose tissue macrophage polarization. J Clin Invest, 2007. 117(1): p. 175-84.

33. Srikakulapu, P., et al., Perivascular Adipose Tissue Harbors Atheroprotective IgMProducing B Cells. Front Physiol, 2017. 8: p. 719.

34. Hussaarts, L., et al., Chronic helminth infection and helminth-derived egg antigens promote adipose tissue M2 macrophages and improve insulin sensitivity in obese mice. FASEB J, 2015. 29(7): p. 3027-39.

35. Withers, S.B., et al., Eosinophils are key regulators of perivascular adipose tissue and vascular functionality. Sci Rep, 2017. 7: p. 44571.

36. Wu, D., et al., Eosinophils sustain adipose alternatively activated macrophages associated with glucose homeostasis. Science, 2011. 332(6026): p. 243-7. 
37. Bussey, C.E., et al., Obesity-Related Perivascular Adipose Tissue Damage Is Reversed by Sustained Weight Loss in the Rat. Arterioscler Thromb Vasc Biol, 2016. 36(7): p. 137785.

38. Gil-Ortega, M., et al., Regional differences in perivascular adipose tissue impacting vascular homeostasis. Trends Endocrinol Metab, 2015. 26(7): p. 367-75.

39. Lin, Y., et al., The hyperglycemia-induced inflammatory response in adipocytes: the role of reactive oxygen species. J Biol Chem, 2005. 280(6): p. 4617-26.

40. Trevellin, E., et al., Exercise training induces mitochondrial biogenesis and glucose uptake in subcutaneous adipose tissue through eNOS-dependent mechanisms. Diabetes, 2014. 63(8): p. 2800-11.

41. Bostrom, P., et al., A PGC1-alpha-dependent myokine that drives brown-fat-like development of white fat and thermogenesis. Nature, 2012. 481(7382): p. 463-8.

42. Soltis, E.E. and L.A. Cassis, Influence of perivascular adipose tissue on rat aortic smooth muscle responsiveness. Clin Exp Hypertens A, 1991. 13(2): p. 277-96.

43. Lohn, M., et al., Periadventitial fat releases a vascular relaxing factor. FASEB J, 2002. 16(9): p. 1057-63.

44. Gollasch, M., Adipose-Vascular Coupling and Potential Therapeutics. Annu Rev Pharmacol Toxicol, 2017. 57: p. 417-436.

45. Gao, Y.J., et al., Modulation of vascular function by perivascular adipose tissue: the role of endothelium and hydrogen peroxide. Br J Pharmacol, 2007. 151(3): p. 323-31.

46. Koh, E.H., et al., eNOS plays a major role in adiponectin synthesis in adipocytes. Am J Physiol Endocrinol Metab, 2010. 298(4): p. E846-53. 
47. Murdaugh, L.S., et al., Compositional studies of human RPE lipofuscin: mechanisms of molecular modifications. J Mass Spectrom, 2011. 46(1): p. 90-5.

48. Ohashi, K., et al., Adiponectin promotes macrophage polarization toward an antiinflammatory phenotype. J Biol Chem, 2010. 285(9): p. 6153-60.

49. Okamoto, Y., et al., Adiponectin inhibits the production of CXC receptor 3 chemokine ligands in macrophages and reduces T-lymphocyte recruitment in atherogenesis. Circ Res, 2008. 102(2): p. 218-25.

50. Mandal, P., et al., The anti-inflammatory effects of adiponectin are mediated via a heme oxygenase-1-dependent pathway in rat Kupffer cells. Hepatology, 2010. 51(4): p. 1420-9.

51. Raychaudhuri, B., et al., Interleukin 10 (IL-10)-mediated inhibition of inflammatory cytokine production by human alveolar macrophages. Cytokine, 2000. 12(9): p. 1348-55.

52. Kamizato, M., et al., Interleukin 10 inhibits interferon gamma- and tumor necrosis factor alpha-stimulated activation of NADPH oxidase 1 in human colonic epithelial cells and the mouse colon. J Gastroenterol, 2009. 44(12): p. 1172-84.

53. Fesus, G., et al., Adiponectin is a novel humoral vasodilator. Cardiovasc Res, 2007. 75(4): p. 719-27.

54. Greenstein, A.S., et al., Local inflammation and hypoxia abolish the protective anticontractile properties of perivascular fat in obese patients. Circulation, 2009. 119(12): p. 1661-70.

55. Cheng, K.K., et al., Adiponectin-induced endothelial nitric oxide synthase activation and nitric oxide production are mediated by APPL1 in endothelial cells. Diabetes, 2007. 56(5): p. 1387-94. 
56. Hattori, Y., et al., Globular adiponectin upregulates nitric oxide production in vascular endothelial cells. Diabetologia, 2003. 46(11): p. 1543-9.

57. Wang, Z.V. and P.E. Scherer, Adiponectin, cardiovascular function, and hypertension. Hypertension, 2008. 51(1): p. 8-14.

58. Zhu, W., et al., Vascular effects of adiponectin: molecular mechanisms and potential therapeutic intervention. Clin Sci (Lond), 2008. 114(5): p. 361-74.

59. Zemse, S.M., et al., Interleukin-10 inhibits the in vivo and in vitro adverse effects of TNFalpha on the endothelium of murine aorta. Am J Physiol Heart Circ Physiol, 2010. 299(4): p. H1160-7.

60. Cattaruzza, M., et al., Interleukin-10 induction of nitric-oxide synthase expression attenuates CD40-mediated interleukin-12 synthesis in human endothelial cells. J Biol Chem, 2003. 278(39): p. 37874-80.

61. Engeli, S., et al., The adipose-tissue renin-angiotensin-aldosterone system: role in the metabolic syndrome? Int J Biochem Cell Biol, 2003. 35(6): p. 807-25.

62. Chappell, M.C., et al., Novel aspects of the renal renin-angiotensin system: angiotensin(1-7), ACE2 and blood pressure regulation. Contrib Nephrol, 2004. 143: p. 77-89.

63. Lee, R.M., et al., Endothelium-dependent relaxation factor released by perivascular adipose tissue. J Hypertens, 2009. 27(4): p. 782-90.

64. Aguilar, M., et al., Prevalence of the metabolic syndrome in the United States, 20032012. JAMA, 2015. 313(19): p. 1973-4.

65. Wilson, P.W., et al., Metabolic syndrome as a precursor of cardiovascular disease and type 2 diabetes mellitus. Circulation, 2005. 112(20): p. 3066-72. 
66. Brooks, S.D., et al., Metabolic syndrome impairs reactivity and wall mechanics of cerebral resistance arteries in obese Zucker rats. Am J Physiol Heart Circ Physiol, 2015. 309(11): p. H1846-59.

67. Donley, D.A., et al., Aerobic exercise training reduces arterial stiffness in metabolic syndrome. J Appl Physiol (1985), 2014. 116(11): p. 1396-404.

68. Frisbee, J.C., et al., Distinct temporal phases of microvascular rarefaction in skeletal muscle of obese Zucker rats. Am J Physiol Heart Circ Physiol, 2014. 307(12): p. H171428.

69. Schillaci, G., et al., Metabolic syndrome is associated with aortic stiffness in untreated essential hypertension. Hypertension, 2005. 45(6): p. 1078-82.

70. Harvey, A., A.C. Montezano, and R.M. Touyz, Vascular biology of ageing-Implications in hypertension. J Mol Cell Cardiol, 2015. 83: p. 112-21.

71. Protogerou, A., et al., Blood pressure response under chronic antihypertensive drug therapy: the role of aortic stiffness in the REASON (Preterax in Regression of Arterial Stiffness in a Controlled Double-Blind) study. J Am Coll Cardiol, 2009. 53(5): p. 445-51.

72. Najjar, S.S., et al., Pulse wave velocity is an independent predictor of the longitudinal increase in systolic blood pressure and of incident hypertension in the Baltimore Longitudinal Study of Aging. J Am Coll Cardiol, 2008. 51(14): p. 1377-83.

73. Fleenor, B.S., Large elastic artery stiffness with aging: novel translational mechanisms and interventions. Aging Dis, 2013. 4(2): p. 76-83.

74. Vafaie, F., et al., Collagenase-resistant collagen promotes mouse aging and vascular cell senescence. Aging Cell, 2014. 13(1): p. 121-30. 
75. Semba, R.D., et al., Serum carboxymethyl-lysine, an advanced glycation end product, is associated with increased aortic pulse wave velocity in adults. Am J Hypertens, 2009. 22(1): p. 74-9.

76. Marchesi, C., et al., Endothelial nitric oxide synthase uncoupling and perivascular adipose oxidative stress and inflammation contribute to vascular dysfunction in a rodent model of metabolic syndrome. Hypertension, 2009. 54(6): p. 1384-92.

77. Ketonen, J., et al., Periadventitial adipose tissue promotes endothelial dysfunction via oxidative stress in diet-induced obese C57Bl/6 mice. Circ J, 2010. 74(7): p. 1479-87.

78. van Dam, A.D., et al., Targeting white, brown and perivascular adipose tissue in atherosclerosis development. Eur J Pharmacol, 2017.

79. Pasarica, M., et al., Reduced adipose tissue oxygenation in human obesity: evidence for rarefaction, macrophage chemotaxis, and inflammation without an angiogenic response. Diabetes, 2009. 58(3): p. 718-25.

80. Fuster, J.J., et al., Obesity-Induced Changes in Adipose Tissue Microenvironment and Their Impact on Cardiovascular Disease. Circ Res, 2016. 118(11): p. 1786-807.

81. Hosogai, N., et al., Adipose tissue hypoxia in obesity and its impact on adipocytokine dysregulation. Diabetes, 2007. 56(4): p. 901-11.

82. Cinti, S., et al., Adipocyte death defines macrophage localization and function in adipose tissue of obese mice and humans. J Lipid Res, 2005. 46(11): p. 2347-55.

83. Murray, P.J. and T.A. Wynn, Protective and pathogenic functions of macrophage subsets. Nat Rev Immunol, 2011. 11(11): p. 723-37.

84. Wynn, T.A., A. Chawla, and J.W. Pollard, Macrophage biology in development, homeostasis and disease. Nature, 2013. 496(7446): p. 445-55. 
85. Shirai, T., et al., Macrophages in vascular inflammation--From atherosclerosis to vasculitis. Autoimmunity, 2015. 48(3): p. 139-51.

86. Wang, H., et al., Obesity-induced endothelial dysfunction is prevented by deficiency of Pselectin glycoprotein ligand-1. Diabetes, 2012. 61(12): p. 3219-27.

87. Ping, D., P.L. Jones, and J.M. Boss, TNF regulates the in vivo occupancy of both distal and proximal regulatory regions of the MCP-1/JE gene. Immunity, 1996. 4(5): p. 455-69.

88. Gaultier, A., et al., Regulation of tumor necrosis factor receptor-1 and the IKK-NFkappaB pathway by LDL receptor-related protein explains the antiinflammatory activity of this receptor. Blood, 2008. 111(11): p. 5316-25.

89. Papa, S., et al., Early modulation of pro-inflammatory microglia by minocycline loaded nanoparticles confers long lasting protection after spinal cord injury. Biomaterials, 2016. 75: p. 13-24.

90. Trayhurn, P., B. Wang, and I.S. Wood, Hypoxia in adipose tissue: a basis for the dysregulation of tissue function in obesity? Br J Nutr, 2008. 100(2): p. 227-35.

91. Snodgrass, R.G., et al., Hypoxia Potentiates Palmitate-induced Pro-inflammatory Activation of Primary Human Macrophages. J Biol Chem, 2016. 291(1): p. 413-24.

92. Delbosc, S., et al., Involvement of oxidative stress and NADPH oxidase activation in the development of cardiovascular complications in a model of insulin resistance, the fructose-fed rat. Atherosclerosis, 2005. 179(1): p. 43-9.

93. Rebolledo, A., et al., Early alterations in vascular contractility associated to changes in fatty acid composition and oxidative stress markers in perivascular adipose tissue. Cardiovasc Diabetol, 2010. 9: p. 65. 
94. Gil-Ortega, M., et al., Adaptative nitric oxide overproduction in perivascular adipose tissue during early diet-induced obesity. Endocrinology, 2010. 151(7): p. 3299-306.

95. Sakamoto, T., et al., Inflammation induced by RAW macrophages suppresses UCP1 mRNA induction via ERK activation in 10T1/2 adipocytes. Am J Physiol Cell Physiol, 2013. 304(8): p. C729-38.

96. Aghamohammadzadeh, R., et al., Effects of Obesity on Perivascular Adipose Tissue Vasorelaxant Function: Nitric Oxide, Inflammation and Elevated Systemic Blood Pressure. J Vasc Res, 2015. 52(5): p. 299-305.

97. Gil-Ortega, M., et al., Imbalance between pro and anti-oxidant mechanisms in perivascular adipose tissue aggravates long-term high-fat diet-derived endothelial dysfunction. PLoS One, 2014. 9(4): p. e95312.

98. da Costa, R.M., et al., Increased mitochondrial ROS generation mediates the loss of the anti-contractile effects of perivascular adipose tissue in high-fat diet obese mice. $\mathrm{Br} \mathrm{J}$ Pharmacol, 2017. 174(20): p. 3527-3541.

99. Chen, J.Y., et al., Increased aortic stiffness and attenuated lysyl oxidase activity in obesity. Arterioscler Thromb Vasc Biol, 2013. 33(4): p. 839-46.

100. Gao, Y.J., et al., Perivascular adipose tissue promotes vasoconstriction: the role of superoxide anion. Cardiovasc Res, 2006. 71(2): p. 363-73.

101. Xia, N., et al., Uncoupling of Endothelial Nitric Oxide Synthase in Perivascular Adipose Tissue of Diet-Induced Obese Mice. Arterioscler Thromb Vasc Biol, 2016. 36(1): p. 7885.

102. Bedard, K. and K.H. Krause, The NOX family of ROS-generating NADPH oxidases: physiology and pathophysiology. Physiol Rev, 2007. 87(1): p. 245-313. 
103. Sanchez C, A.V., Grino M, Tanguy S, Long Term High-Fat Diet-Induced Modification of Vascular Wall and Perivascular Adipose Tissue-Mediated Oxidative Stress: Consequences for Endothelium-Independent Vascular Function in Rats. Int J Clin Cardiol, 2017. 4(2).

104. Luo, S., et al., Molecular mechanisms of endothelial NO synthase uncoupling. Curr Pharm Des, 2014. 20(22): p. 3548-53.

105. Sekhar, S., et al., Attenuation of reactive oxygen/nitrogen species with suppression of inducible nitric oxide synthase expression in RAW 264.7 macrophages by bark extract of Buchanania lanzan. Pharmacogn Mag, 2015. 11(42): p. 283-91.

106. Hayashi, A., et al., In vitro degradation of tropoelastin by reactive oxygen species. Arch Dermatol Res, 1998. 290(9): p. 497-500.

107. Fleenor, B.S., et al., Superoxide signaling in perivascular adipose tissue promotes agerelated artery stiffness. Aging Cell, 2014. 13(3): p. 576-8.

108. Diaz-Ruiz, A., et al., Proteasome Dysfunction Associated to Oxidative Stress and Proteotoxicity in Adipocytes Compromises Insulin Sensitivity in Human Obesity. Antioxid Redox Signal, 2015. 23(7): p. 597-612.

109. Ehlinger, A. and K.J. Walters, Structural insights into proteasome activation by the 19S regulatory particle. Biochemistry, 2013. 52(21): p. 3618-28.

110. Rabl, J., et al., Mechanism of gate opening in the 20 S proteasome by the proteasomal ATPases. Mol Cell, 2008. 30(3): p. 360-8.

111. Smith, D.M., et al., ATP binds to proteasomal ATPases in pairs with distinct functional effects, implying an ordered reaction cycle. Cell, 2011. 144(4): p. 526-38. 
112. Wang, X., et al., Regulation of the 26 S proteasome complex during oxidative stress. Sci Signal, 2010. 3(151): p. ra88.

113. Gregersen, N. and P. Bross, Protein misfolding and cellular stress: an overview. Methods Mol Biol, 2010. 648: p. 3-23.

114. Otoda, T., et al., Proteasome dysfunction mediates obesity-induced endoplasmic reticulum stress and insulin resistance in the liver. Diabetes, 2013. 62(3): p. 811-24.

115. Ghosh, A.K., et al., Elevated Endoplasmic Reticulum Stress Response Contributes to Adipose Tissue Inflammation in Aging. J Gerontol A Biol Sci Med Sci, 2015. 70(11): p. $1320-9$.

116. Hohn, A., J. Konig, and T. Jung, Metabolic Syndrome, Redox State, and the Proteasomal System. Antioxid Redox Signal, 2016. 25(16): p. 902-917.

117. Gloire, G., S. Legrand-Poels, and J. Piette, NF-kappaB activation by reactive oxygen species: fifteen years later. Biochem Pharmacol, 2006. 72(11): p. 1493-505.

118. Kim, Y.S., et al., TNF-induced activation of the Nox1 NADPH oxidase and its role in the induction of necrotic cell death. Mol Cell, 2007. 26(5): p. 675-87.

119. Li, J.M., et al., Acute tumor necrosis factor alpha signaling via NADPH oxidase in microvascular endothelial cells: role of 47 phox phosphorylation and binding to TRAF4. Mol Cell Biol, 2005. 25(6): p. 2320-30.

120. Fain, J.N., S.W. Bahouth, and A.K. Madan, TNFalpha release by the nonfat cells of human adipose tissue. Int J Obes Relat Metab Disord, 2004. 28(4): p. 616-22.

121. Virdis, A., et al., Tumour necrosis factor-alpha participates on the endothelin-1/nitric oxide imbalance in small arteries from obese patients: role of perivascular adipose tissue. Eur Heart J, 2015. 36(13): p. 784-94. 
122. Sen, C.K. and L. Packer, Antioxidant and redox regulation of gene transcription. FASEB J, 1996. 10(7): p. 709-20.

123. Goh, J., K.P. Goh, and A. Abbasi, Exercise and Adipose Tissue Macrophages: New Frontiers in Obesity Research? Front Endocrinol (Lausanne), 2016. 7: p. 65.

124. Didion, S.P., et al., Endogenous interleukin-10 inhibits angiotensin II-induced vascular dysfunction. Hypertension, 2009. 54(3): p. 619-24.

125. Rodriguez, A., et al., The inhibitory effect of leptin on angiotensin II-induced vasoconstriction in vascular smooth muscle cells is mediated via a nitric oxide-dependent mechanism. Endocrinology, 2007. 148(1): p. 324-31.

126. Shek, E.W., M.W. Brands, and J.E. Hall, Chronic leptin infusion increases arterial pressure. Hypertension, 1998. 31(1 Pt 2): p. 409-14.

127. Turner, N.A., et al., Mechanism of TNFalpha-induced IL-1alpha, IL-1beta and IL-6 expression in human cardiac fibroblasts: effects of statins and thiazolidinediones. Cardiovasc Res, 2007. 76(1): p. 81-90.

128. Gurjar, M.V., et al., Role of reactive oxygen species in IL-1 beta-stimulated sustained ERK activation and MMP-9 induction. Am J Physiol Heart Circ Physiol, 2001. 281(6): p. H2568-74.

129. Saperstein, S., et al., IL-1beta augments TNF-alpha-mediated inflammatory responses from lung epithelial cells. J Interferon Cytokine Res, 2009. 29(5): p. 273-84.

130. Salajegheh, M., et al., Upregulation of thrombospondin-1(TSP-1) and its binding partners, CD36 and CD47, in sporadic inclusion body myositis. J Neuroimmunol, 2007. 187(1-2): p. 166-74. 
131. Isenberg, J.S., et al., Regulation of nitric oxide signalling by thrombospondin 1: implications for anti-angiogenic therapies. Nat Rev Cancer, 2009. 9(3): p. 182-94.

132. Mandler, W.K., et al., Thrombospondin-1 mediates multi-walled carbon nanotube induced impairment of arteriolar dilation. Nanotoxicology, 2017. 11(1): p. 112-122.

133. Rausch, M.E., et al., Obesity in C57BL/6J mice is characterized by adipose tissue hypoxia and cytotoxic T-cell infiltration. Int J Obes (Lond), 2008. 32(3): p. 451-63.

134. O'Rourke, R.W., et al., Hypoxia-induced inflammatory cytokine secretion in human adipose tissue stromovascular cells. Diabetologia, 2011. 54(6): p. 1480-90.

135. Bouloumie, A., et al., Adipocyte produces matrix metalloproteinases 2 and 9: involvement in adipose differentiation. Diabetes, 2001. 50(9): p. 2080-6.

136. Chakraborti, S., et al., Regulation of matrix metalloproteinases: an overview. Mol Cell Biochem, 2003. 253(1-2): p. 269-85.

137. Yasmin, et al., Matrix metalloproteinase-9 (MMP-9), MMP-2, and serum elastase activity are associated with systolic hypertension and arterial stiffness. Arterioscler Thromb Vasc Biol, 2005. 25(2): p. 372.

138. $\mathrm{Wu}, \mathrm{H} . T$., et al., Identifying the regulative role of NF-kappaB binding sites within promoter region of human matrix metalloproteinase 9 (mmp-9) by TNF-alpha induction. Appl Biochem Biotechnol, 2013. 169(2): p. 438-49.

139. Donnini, S., et al., ERKI-2 and $p 38$ MAPK regulate MMP/TIMP balance and function in response to thrombospondin-1 fragments in the microvascular endothelium. Life Sci, 2004. 74(24): p. 2975-85.

140. Brown, R.D., et al., Cytokines regulate matrix metalloproteinases and migration in cardiac fibroblasts. Biochem Biophys Res Commun, 2007. 362(1): p. 200-5. 
141. Gearing, A.J., et al., Processing of tumour necrosis factor-alpha precursor by metalloproteinases. Nature, 1994. 370(6490): p. 555-7.

142. Schonbeck, U., F. Mach, and P. Libby, Generation of biologically active IL-1 beta by matrix metalloproteinases: a novel caspase-1-independent pathway of IL-1 beta processing. J Immunol, 1998. 161(7): p. 3340-6.

143. Blomkalns, A.L., et al., CD14 directs adventitial macrophage precursor recruitment: role in early abdominal aortic aneurysm formation. J Am Heart Assoc, 2013. 2(2): p. $\mathrm{e} 000065$.

144. Ivanov, II, et al., The orphan nuclear receptor RORgammat directs the differentiation program of proinflammatory IL-17+ Thelper cells. Cell, 2006. 126(6): p. 1121-33.

145. Hung, M.J., et al., Interleukin-6 inhibits endothelial nitric oxide synthase activation and increases endothelial nitric oxide synthase binding to stabilized caveolin-1 in human vascular endothelial cells. J Hypertens, 2010. 28(5): p. 940-51.

146. Wassmann, S., et al., Interleukin-6 induces oxidative stress and endothelial dysfunction by overexpression of the angiotensin II type 1 receptor. Circ Res, 2004. 94(4): p. 534-41.

147. Du, B., et al., Aortic perivascular adipose-derived interleukin-6 contributes to arterial stiffness in low-density lipoprotein receptor deficient mice. Am J Physiol Heart Circ Physiol, 2015. 308(11): p. H1382-90.

148. Callera, G.E., et al., Aldosterone activates vascular p38MAP kinase and NADPH oxidase via c-Src. Hypertension, 2005. 45(4): p. 773-9.

149. Thatcher, S., et al., The adipose renin-angiotensin system: role in cardiovascular disease. Mol Cell Endocrinol, 2009. 302(2): p. 111-7. 
150. Briones, A.M., et al., Adipocytes produce aldosterone through calcineurin-dependent signaling pathways: implications in diabetes mellitus-associated obesity and vascular dysfunction. Hypertension, 2012. 59(5): p. 1069-78.

151. Briet, M., et al., Aldosterone-Induced Vascular Remodeling and Endothelial Dysfunction Require Functional Angiotensin Type 1a Receptors. Hypertension, 2016. 67(5): p. 897905.

152. Guzik, T.J., et al., Role of the $T$ cell in the genesis of angiotensin II induced hypertension and vascular dysfunction. J Exp Med, 2007. 204(10): p. 2449-60.

153. Lu, C., et al., Alterations in perivascular adipose tissue structure and function in hypertension. Eur J Pharmacol, 2011. 656(1-3): p. 68-73.

154. Hartupee, J., et al., IL-17 enhances chemokine gene expression through mRNA stabilization. J Immunol, 2007. 179(6): p. 4135-41.

155. Kossmann, S., et al., Angiotensin II-induced vascular dysfunction depends on interferongamma-driven immune cell recruitment and mutual activation of monocytes and NKcells. Arterioscler Thromb Vasc Biol, 2013. 33(6): p. 1313-9.

156. Galvez, B., et al., Perivascular adipose tissue and mesenteric vascular function in spontaneously hypertensive rats. Arterioscler Thromb Vasc Biol, 2006. 26(6): p. 1297302.

157. Kondo, T., et al., Cutting edge: Phenotypic characterization and differentiation of human CD8+ T cells producing IL-17. J Immunol, 2009. 182(4): p. 1794-8.

158. Pietrowski, E., et al., Pro-inflammatory effects of interleukin-17A on vascular smooth muscle cells involve $N A D(P) H$ - oxidase derived reactive oxygen species. J Vasc Res, 2011. 48(1): p. 52-8. 
159. National Survey on Drug Use and Health (U.S.) and United States. Substance Abuse and Mental Health Services Administration. Office of Applied Studies., Results from the ... National Survey on Drug Use and Health. National findings, in National Survey on Drug Use and Health series. 2015, Dept. of Health and Human Services, Substance Abuse and Mental Health Services Administration, Office of Applied Studies: Rockville, MD. p. 7 volumes.

160. Van der Kooy, K., et al., Depression and the risk for cardiovascular diseases: systematic review and meta analysis. Int J Geriatr Psychiatry, 2007. 22(7): p. 613-26.

161. Gullette, E.C., et al., Effects of mental stress on myocardial ischemia during daily life. JAMA, 1997. 277(19): p. 1521-6.

162. Seldenrijk, A., et al., Depression, anxiety, and arterial stiffness. Biol Psychiatry, 2011. 69(8): p. 795-803.

163. Mattace-Raso, F.U., et al., Arterial stiffness and risk of coronary heart disease and stroke: the Rotterdam Study. Circulation, 2006. 113(5): p. 657-63.

164. Rajagopalan, S., et al., Abnormal brachial artery flow-mediated vasodilation in young adults with major depression. Am J Cardiol, 2001. 88(2): p. 196-8, A7.

165. Golbidi, S., J.C. Frisbee, and I. Laher, Chronic stress impacts the cardiovascular system: animal models and clinical outcomes. Am J Physiol Heart Circ Physiol, 2015. 308(12): p. H1476-98.

166. Carey, M., et al., Prevalence of comorbid depression and obesity in general practice: a cross-sectional survey. Br J Gen Pract, 2014. 64(620): p. e122-7.

167. Pratt, L.A. and D.J. Brody, Depression and obesity in the U.S. adult household population, 2005-2010. NCHS Data Brief, 2014(167): p. 1-8. 
168. Yang, J.L., et al., The Effects of High-fat-diet Combined with Chronic Unpredictable Mild Stress on Depression-like Behavior and Leptin/LepRb in Male Rats. Sci Rep, 2016. 6: p. 35239.

169. Mineur, Y.S., C. Belzung, and W.E. Crusio, Effects of unpredictable chronic mild stress on anxiety and depression-like behavior in mice. Behav Brain Res, 2006. 175(1): p. 4350.

170. Willner, P., Validity, reliability and utility of the chronic mild stress model of depression: a 10-year review and evaluation. Psychopharmacology (Berl), 1997. 134(4): p. 319-29.

171. Olini, N., et al., Chronic social stress leads to altered sleep homeostasis in mice. Behav Brain Res, 2017. 327: p. 167-173.

172. Gronli, J., et al., Chronic mild stress affects sucrose intake and sleep in rats. Behav Brain Res, 2004. 150(1-2): p. 139-47.

173. Hill, M.N., et al., Neurobiology of chronic mild stress: parallels to major depression. Neurosci Biobehav Rev, 2012. 36(9): p. 2085-117.

174. Willner, P., et al., Reduction of sucrose preference by chronic unpredictable mild stress, and its restoration by a tricyclic antidepressant. Psychopharmacology (Berl), 1987. 93(3): p. 358-64.

175. d'Audiffret, A.C., et al., Depressive behavior and vascular dysfunction: a link between clinical depression and vascular disease? J Appl Physiol (1985), 2010. 108(5): p. 104151.

176. Wallerath, T., et al., Down-regulation of the expression of endothelial NO synthase is likely to contribute to glucocorticoid-mediated hypertension. Proc Natl Acad Sci U S A, 1999. 96(23): p. 13357-62. 
177. Rogers, K.M., et al., Inhibitory effect of glucocorticoid on coronary artery endothelial function. Am J Physiol Heart Circ Physiol, 2002. 283(5): p. H1922-8.

178. Liu, Y., et al., Glucocorticoid response elements and 11 beta-hydroxysteroid dehydrogenases in the regulation of endothelial nitric oxide synthase expression. Cardiovasc Res, 2009. 81(1): p. 140-7.

179. Iuchi, T., et al., Glucocorticoid excess induces superoxide production in vascular endothelial cells and elicits vascular endothelial dysfunction. Circ Res, 2003. 92(1): p. 81-7.

180. Zafir, A. and N. Banu, Induction of oxidative stress by restraint stress and corticosterone treatments in rats. Indian J Biochem Biophys, 2009. 46(1): p. 53-8.

181. Cosentino, F. and T.F. Luscher, Tetrahydrobiopterin and endothelial nitric oxide synthase activity. Cardiovasc Res, 1999. 43(2): p. 274-8.

182. Galougahi, K.K., et al., Glutathionylation mediates angiotensin II-induced eNOS uncoupling, amplifying NADPH oxidase-dependent endothelial dysfunction. J Am Heart Assoc, 2014. 3(2): p. e000731.

183. McIntosh, L.J., K.E. Hong, and R.M. Sapolsky, Glucocorticoids may alter antioxidant enzyme capacity in the brain: baseline studies. Brain Res, 1998. 791(1-2): p. 209-14.

184. Orzechowski, A., et al., Excess of glucocorticoids impairs whole-body antioxidant status in young rats. relation to the effect of dexamethasone in soleus muscle and spleen. Horm Metab Res, 2000. 32(5): p. 174-80.

185. Hafezi-Moghadam, A., et al., Acute cardiovascular protective effects of corticosteroids are mediated by non-transcriptional activation of endothelial nitric oxide synthase. Nat Med, 2002. 8(5): p. 473-9. 
186. Lu, X.T., et al., Unpredictable chronic mild stress promotes atherosclerosis in high cholesterol-fed rabbits. Psychosom Med, 2012. 74(6): p. 604-11.

187. Harvey, R.E., et al., Influence of sympathetic nerve activity on aortic hemodynamics and pulse wave velocity in women. Am J Physiol Heart Circ Physiol, 2017. 312(2): p. H340H346.

188. Clamage, D.M., et al., Effects of psychosocial stimuli on plasma renin activity in rats. Am J Physiol, 1976. 231(4): p. 1290-4.

189. Saxena, P.R., Interaction between the renin-angiotensin-aldosterone and sympathetic nervous systems. J Cardiovasc Pharmacol, 1992. 19 Suppl 6: p. S80-8.

190. Goldsmith, S.R., Interactions between the sympathetic nervous system and the RAAS in heart failure. Curr Heart Fail Rep, 2004. 1(2): p. 45-50.

191. Touyz, R.M., Reactive oxygen species and angiotensin II signaling in vascular cells -implications in cardiovascular disease. Braz J Med Biol Res, 2004. 37(8): p. 1263-73.

192. Wang, D., et al., Role of oxidative stress in endothelial dysfunction and enhanced responses to angiotensin II of afferent arterioles from rabbits infused with angiotensin II.

J Am Soc Nephrol, 2003. 14(11): p. 2783-9.

193. Zuo, L., et al., Caveolin-1 is essential for activation of Racl and NAD(P)H oxidase after angiotensin II type 1 receptor stimulation in vascular smooth muscle cells: role in redox signaling and vascular hypertrophy. Arterioscler Thromb Vasc Biol, 2005. 25(9): p. 1824-30.

194. Lassegue, B., et al., Novel gp91(phox) homologues in vascular smooth muscle cells : nox 1 mediates angiotensin II-induced superoxide formation and redox-sensitive signaling pathways. Circ Res, 2001. 88(9): p. 888-94. 
195. Miyata, K., et al., Aldosterone stimulates reactive oxygen species production through activation of NADPH oxidase in rat mesangial cells. J Am Soc Nephrol, 2005. 16(10): p. 2906-12.

196. Grote, K., et al., Critical role for p47phox in renin-angiotensin system activation and blood pressure regulation. Cardiovasc Res, 2006. 71(3): p. 596-605.

197. Tsuji, H., et al., Involvement of renin-angiotensin-aldosterone system in calcium oxalate crystal induced activation of NADPH oxidase and renal cell injury. World J Urol, 2016. 34(1): p. 89-95.

198. Strack, A.M., M.J. Bradbury, and M.F. Dallman, Corticosterone decreases nonshivering thermogenesis and increases lipid storage in brown adipose tissue. Am J Physiol, 1995. 268(1 Pt 2): p. R183-91.

199. Karagiannides, I., et al., Chronic unpredictable stress regulates visceral adipocytemediated glucose metabolism and inflammatory circuits in male rats. Physiol Rep, 2014. 2(5): p. e00284.

200. Pereira, V.H., et al., Glucose intolerance after chronic stress is related with downregulated PPAR-gamma in adipose tissue. Cardiovasc Diabetol, 2016. 15(1): p. 114.

201. Auphan, N., et al., Immunosuppression by glucocorticoids: inhibition of NF-kappa B activity through induction of I kappa B synthesis. Science, 1995. 270(5234): p. 286-90.

202. Coutinho, A.E. and K.E. Chapman, The anti-inflammatory and immunosuppressive effects of glucocorticoids, recent developments and mechanistic insights. Mol Cell Endocrinol, 2011. 335(1): p. 2-13. 
203. Avitsur, R., J.L. Stark, and J.F. Sheridan, Social stress induces glucocorticoid resistance in subordinate animals. Horm Behav, 2001. 39(4): p. 247-57.

204. Miller, G.E., et al., A functional genomic fingerprint of chronic stress in humans: blunted glucocorticoid and increased NF-kappaB signaling. Biol Psychiatry, 2008. 64(4): p. 26672.

205. Stark, J.L., et al., Social stress induces glucocorticoid resistance in macrophages. Am J Physiol Regul Integr Comp Physiol, 2001. 280(6): p. R1799-805.

206. Spengler, R.N., et al., Stimulation of alpha-adrenergic receptor augments the production of macrophage-derived tumor necrosis factor. J Immunol, 1990. 145(5): p. 1430-4.

207. Tsuchiya, K., et al., Angiotensin II induces monocyte chemoattractant protein-1 expression via a nuclear factor-kappaB-dependent pathway in rat preadipocytes. Am J Physiol Endocrinol Metab, 2006. 291(4): p. E771-8.

208. Kalra, D., N. Sivasubramanian, and D.L. Mann, Angiotensin II induces tumor necrosis factor biosynthesis in the adult mammalian heart through a protein kinase C-dependent pathway. Circulation, 2002. 105(18): p. 2198-205.

209. Wolf, G., et al., Angiotensin II activates nuclear transcription factor-kappaB through AT1 and AT2 receptors. Kidney Int, 2002. 61(6): p. 1986-95.

210. Kershaw, E.E. and J.S. Flier, Adipose tissue as an endocrine organ. J Clin Endocrinol Metab, 2004. 89(6): p. 2548-56.

211. DeVallance, E., et al., Effect of chronic stress on running wheel activity in mice. PLoS One, 2017. 12(9): p. e0184829. 
212. Cornelissen, V.A. and R.H. Fagard, Effects of endurance training on blood pressure, blood pressure-regulating mechanisms, and cardiovascular risk factors. Hypertension, 2005. 46(4): p. 667-75.

213. Miller, T.D., G.J. Balady, and G.F. Fletcher, Exercise and its role in the prevention and rehabilitation of cardiovascular disease. Ann Behav Med, 1997. 19(3): p. 220-9.

214. Edwards, D.G., et al., Effect of exercise training on endothelial function in men with coronary artery disease. Am J Cardiol, 2004. 93(5): p. 617-20.

215. Hambrecht, R., et al., Effect of exercise on coronary endothelial function in patients with coronary artery disease. N Engl J Med, 2000. 342(7): p. 454-60.

216. North, T.C., P. McCullagh, and Z.V. Tran, Effect of exercise on depression. Exerc Sport Sci Rev, 1990. 18: p. 379-415.

217. Mueller, P.J., Exercise training and sympathetic nervous system activity: evidence for physical activity dependent neural plasticity. Clin Exp Pharmacol Physiol, 2007. 34(4): p. $377-84$.

218. Hambrecht, R., et al., Regular physical activity improves endothelial function in patients with coronary artery disease by increasing phosphorylation of endothelial nitric oxide synthase. Circulation, 2003. 107(25): p. 3152-8.

219. Kojda, G., et al., Dysfunctional regulation of endothelial nitric oxide synthase (eNOS) expression in response to exercise in mice lacking one eNOS gene. Circulation, 2001. 103(23): p. 2839-44.

220. Laurindo, F.R., et al., Vascular free radical release. Ex vivo and in vivo evidence for a flow-dependent endothelial mechanism. Circ Res, 1994. 74(4): p. 700-9. 
221. Drummond, G.R., et al., Transcriptional and posttranscriptional regulation of endothelial nitric oxide synthase expression by hydrogen peroxide. Circ Res, 2000. 86(3): p. 347-54.

222. Zhang, Q.J., et al., Endothelial nitric oxide synthase phosphorylation in treadmillrunning mice: role of vascular signalling kinases. J Physiol, 2009. 587(Pt 15): p. 391120.

223. Sindler, A.L., et al., Effects of ageing and exercise training on eNOS uncoupling in skeletal muscle resistance arterioles. J Physiol, 2009. 587(Pt 15): p. 3885-97.

224. Widder, J.D., et al., Regulation of tetrahydrobiopterin biosynthesis by shear stress. Circ Res, 2007. 101(8): p. 830-8.

225. Collier, S.R., et al., Effect of 4 weeks of aerobic or resistance exercise training on arterial stiffness, blood flow and blood pressure in pre- and stage-1 hypertensives. J Hum Hypertens, 2008. 22(10): p. 678-86.

226. Edwards, D.G., et al., Effect of exercise training on central aortic pressure wave reflection in coronary artery disease. Am J Hypertens, 2004. 17(6): p. 540-3.

227. Durrant, J.R., et al., Voluntary wheel running restores endothelial function in conduit arteries of old mice: direct evidence for reduced oxidative stress, increased superoxide dismutase activity and down-regulation of NADPH oxidase. J Physiol, 2009. 587(Pt 13): p. 3271-85.

228. Rush, J.W., J.R. Turk, and M.H. Laughlin, Exercise training regulates SOD-1 and oxidative stress in porcine aortic endothelium. Am J Physiol Heart Circ Physiol, 2003. 284(4): p. H1378-87. 
229. Drenth, J.P., et al., Endurance run increases circulating IL-6 and IL-1ra but downregulates ex vivo TNF-alpha and IL-1 beta production. J Appl Physiol (1985), 1995. 79(5): p. 1497-503.

230. Yang, A.L. and H.I. Chen, Chronic exercise reduces adhesion molecules/iNOS expression and partially reverses vascular responsiveness in hypercholesterolemic rabbit aortae. Atherosclerosis, 2003. 169(1): p. 11-7.

231. Ando, J., et al., Shear stress inhibits adhesion of cultured mouse endothelial cells to lymphocytes by downregulating VCAM-1 expression. Am J Physiol, 1994. 267(3 Pt 1): p. C679-87.

232. Kawanishi, N., et al., Exercise training inhibits inflammation in adipose tissue via both suppression of macrophage infiltration and acceleration of phenotypic switching from M1 to M2 macrophages in high-fat-diet-induced obese mice. Exerc Immunol Rev, 2010. 16: p. $105-18$.

233. Oliveira, A.G., et al., Acute exercise induces a phenotypic switch in adipose tissue macrophage polarization in diet-induced obese rats. Obesity (Silver Spring), 2013. 21(12): p. 2545-56.

234. Lira, F.S., et al., Endurance training induces depot-specific changes in IL-10/TNF-alpha ratio in rat adipose tissue. Cytokine, 2009. 45(2): p. 80-5.

235. Gomez-Merino, D., et al., Effects of chronic exercise on cytokine production in white adipose tissue and skeletal muscle of rats. Cytokine, 2007. 40(1): p. 23-9.

236. Balducci, S., et al., Anti-inflammatory effect of exercise training in subjects with type 2 diabetes and the metabolic syndrome is dependent on exercise modalities and independent of weight loss. Nutr Metab Cardiovasc Dis, 2010. 20(8): p. 608-17. 
237. Balducci, S., et al., Changes in physical fitness predict improvements in modifiable cardiovascular risk factors independently of body weight loss in subjects with type 2 diabetes participating in the Italian Diabetes and Exercise Study (IDES). Diabetes Care, 2012. 35(6): p. 1347-54.

238. Hatano, D., et al., Effect of exercise training on the density of endothelial cells in the white adipose tissue of rats. Scand J Med Sci Sports, 2011. 21(6): p. e115-21.

239. Czarkowska-Paczek, B., et al., The influence of physical exercise on the generation of TGF-beta1, PDGF-AA, and VEGF-A in adipose tissue. Eur J Appl Physiol, 2011. 111(5): p. $875-81$.

240. Calhoun, D.A. and K. Sharma, The role of aldosteronism in causing obesity-related cardiovascular risk. Cardiol Clin, 2010. 28(3): p. 517-27.

241. Dall'Asta, C., et al., Effect of weight loss through laparoscopic gastric banding on blood pressure, plasma renin activity and aldosterone levels in morbid obesity. Nutr Metab Cardiovasc Dis, 2009. 19(2): p. 110-4.

242. Engeli, S., et al., Weight loss and the renin-angiotensin-aldosterone system. Hypertension, 2005. 45(3): p. 356-62.

243. Araujo, H.N., et al., Perivascular adipose tissue and vascular responses in healthy trained rats. Life Sci, 2015. 125: p. 79-87. 


\section{Chapter 2: Methods}

tPVAT Preparations: The diaphragm was cut and the aorta with the surround tPVAT was removed immediately and placed in ice cold Krebs Henseleit Buffer $\left(1.18 \mathrm{mM} \mathrm{KH}_{2} \mathrm{PO}_{4}, 1.2 \mathrm{mM}\right.$ $\mathrm{MgSO}_{4} \cdot 7 \mathrm{H}_{2} \mathrm{O}, 4.7 \mathrm{mM} \mathrm{KCl}, 25 \mathrm{mM} \mathrm{NaHCO} 3,118 \mathrm{mM} \mathrm{NaCl}, 5.5 \mathrm{mM}$ glucose, $0.026 \mathrm{mM}$ Ethylenediaminetetraacetic acid (EDTA), $2.5 \mathrm{mM} \mathrm{CaCl}_{2} \cdot 2 \mathrm{H}_{2} \mathrm{O}$, bubbled with $95 \% \mathrm{O}_{2}$ ). tPVAT was carefully removed from the aorta under a dissecting microscope. The tPVAT completely surrounds the aorta however thin stretches of predominantly connective tissue are observed on the anterior and posterior aspects of the aorta. To remove the APVAT two cut were made down the connective tissue to minimize damage to adipocytes and peeled away. After removal, large blood vessels and nervous tissue were removed without major disturbance to the adipose tissue. tPVAT sample were then immediately snap frozen or place in physiological HEPES buffer (43.7 $\mathrm{mM} \mathrm{NaCl}, 80 \mathrm{mM} \mathrm{KCl}, 1.17 \mathrm{mM} \mathrm{MgSO}{ }_{4} \cdot 7 \mathrm{H}_{2} \mathrm{O}, 1.6 \mathrm{mM} \mathrm{NaH}_{2} \mathrm{PO}_{4}, 18 \mathrm{mM} \mathrm{NaHCO}_{3}, 0.03 \mathrm{mM}$ EDTA, $5.5 \mathrm{mM}$ glucose, $5 \mathrm{mM}$ HEPES) for later experimental use.

Treated tPVAT Gene Expression: 50mg sections of tPVAT sections were weighed and incubated at $37^{\circ} \mathrm{C}$ in physiological HEPES buffer $\left(43.7 \mathrm{mM} \mathrm{NaCl}, 80 \mathrm{mM} \mathrm{KCl}, 1.17 \mathrm{mM} \mathrm{MgSO}{ }_{4} \cdot 7 \mathrm{H}_{2} \mathrm{O}\right.$, $1.6 \mathrm{mM} \mathrm{NaH}_{2} \mathrm{PO}_{4}, 18 \mathrm{mM} \mathrm{NaHCO} 3,0.03 \mathrm{mM}$ EDTA, $5.5 \mathrm{mM}$ glucose, $5 \mathrm{mM}$ HEPES) or HEPES buffer containing $4 \mu \mathrm{M}$ TNF $\alpha$ neutralizing antibody (TNF $\alpha$ AB, Catalog \#: AF-510-NA, R\&D systems) at a ratio of $200 \mu \mathrm{g} / \mathrm{mL}$. After 1-hour PVAT was removed and snap frozen. To assess gene expression, tPVAT was homogenized in QIAzol and processed for qPCR using the RNeasy Lipid Tissue Mini Kit (Qiagen), QuantiTect reverse transcription kit (Qiagen 205313). Equal concentrations of cDNA were then loaded into the QIAgility (Qiagen), which mixed $20 \mu \mathrm{L}$ 
PCR reactions with QuantiTect primer assays

Measurement of Reactive Oxygen Species: Dihydroethidium (DHE, Invitrogen D1168) assays were performed on unfixed aortic rings and tPVAT sections to evaluate in situ ROS production. tPVAT was treated with various drugs for 30 minutes then quickly washed in HEPES and placed in a well with an aortic ring. Following the drug incubation $2 \mu$ of stock DHE solution was added to each well to a concentration of $10 \mu \mathrm{M}$ and incubated at $37^{\circ} \mathrm{C}$ for another 30 minutes. Following completion of DHE incubation rings and PVAT were washed in HEPES buffer, placed separately in Optimal Cutting Temperature compound (OCT, Fisher Healthcare ${ }^{\mathrm{TM}}$ TissuePlus ${ }^{\mathrm{TM}}$ O.C.T Compound) and frozen in liquid nitrogen cooled isopentane and stored at $-80^{\circ} \mathrm{C}$. DHE OCT blocks were then cut into $8 \mu \mathrm{m}$ slices using a cryostat at $-22^{\circ} \mathrm{C}$ and transferred to charged slides (Fisherbrand ${ }^{\circledR}$ Superfrost ${ }^{\circledR}$ plus microscope slides) and stained/mounted with DAPI mounting media (VECTORSHEILD antifade mounting media with DAPI, Vector laboratories). Slides were imaged with an EVOS fluorescent microscope (Invitrogen EVOS FL Auto Cell Imaging System), 3 sections per image-treatment, and analyzed in ImageJ as fluorescent density/nucleus, the mean of the 3 images/treatment were used as the mean for each animal. Values were normalized to signal from tempol treatment to eliminate background signal.

Measurement of NO Bioavailability: Aortic NO production was measured by 4-Amino-5Methylamino-2',7'- Difluorofluorescein Diacetate (DAF-FM-DA, Invitrogen) according to the manufacturer's instructions. HEPES buffer was supplemented with L-Arginine $(100 \mu \mathrm{M}$, MP Biomedical Inc. 100736), in order to promote NO production. In order not to contaminate aortic NO production tPVAT exudate was used for the 30-minute incubation. To assess NO in tPVAT 
$10 \mathrm{mg}$ segments were used in separate wells. Aortic rings incubated with the NOS inhibitor LNAME were used to normalize fluorescent readings to eliminate background fluorescence.

Aortic Reactivity: Prior to use for incubation tPVAT segments were sectioned into $200 \mathrm{mg}$ pieces. This weight was determined by preliminary studies in which multiple tissue weights and exudates were compared to aortic rings with intact tPVAT. 200mg incubated in $1 \mathrm{ml}$ HEPES was determined to be the most representative in comparison to intact tPVAT (Fig.1). Additionally, no difference was found between use of the exudate and incubation with tissue (Fig.2). 3mm thoracic aortic rings (cleaned of surrounding tissue, $n=14-16$ ) were rinsed in physiological salt solution and mounted in a myobath chamber between a fixed point and a force transducer (World Precision Instruments) and pre-stretched to 1.5 grams for 1 hour to equilibrate. The organ baths contained Krebs Henseleit Buffer aerated with $95 \% \mathrm{O}_{2}$ and $5 \% \mathrm{CO}_{2}$ at $37^{\circ} \mathrm{C}$. After equilibration, aortic baseline tension was adjusted to a stable 1 gram and vessel viability was checked with $50 \mathrm{mM}$ of $\mathrm{KCl}$ and rings not generating a rapid response were excluded from the study. Aortic relaxation was carried out as previously described ${ }^{1}$. To test EDD, aortic rings were preconstricted with phenylephrine (PE, $1 \times 10^{-7} \mathrm{M}$, Sigma-Aldrich P6126) and a stable tension was reached and recorded. Upon reaching a stable constricted state increasing doses of methacholine $\left(\mathrm{MCh}, 1 \times 10^{-9} \mathrm{M}\right.$ to $\left.1 \times 10^{-5} \mathrm{M}\right)$ were applied to the bath. Relaxation was calculated as $\%$ relaxation for each dose of $\mathrm{MCh}$ from the following equation:

$$
\% \text { relaxation }=\left(\frac{Z-x}{Z-y}\right) \times 100
$$

Where $\mathrm{z}$ is tension after PE $1 \times 10^{-7}, \mathrm{x}$ is the tension following a given does of $\mathrm{MCh}$, and $\mathrm{y}$ is baseline tension. 
Following the initial relaxation curve, the system was washed again and allowed to return to baseline. To test the effect of PVAT on EDD, $200 \mathrm{mg}$ sections of PVAT were incubated in $1 \mathrm{~mL}$ of HEPES buffer both with and without $\mathrm{TNF} \alpha-\mathrm{nAB}$ at $37^{\circ} \mathrm{C}$. The exudate was either snap frozen for use in crossover experiments (LZR tPVAT exudate on OZR aorta and OZR tPVAT exudate on LZR aorta) or used on rings from the same animal as follows. Exudate or 200mg of tPVAT were added to the bath and rings were allowed to incubate for 30 minutes. Pilot studies showed no difference between EDD when aortic rings were incubated with exudate vs. 200mg of tPVAT tissue. Following the incubation, a MCh relaxation curve was carried out as described above. Finally, aortic rings both with and without tPVAT incubation were pre-constricted with PE $1 \times 10^{-7}$ and treated with increasing doses of the endothelial independent dilator sodium nitroprusside (SNP; 1 X10-9 M to 1 X10 $0^{-5} \mathrm{M}$, MP Biomedical 152061 ).

MMP9 Activity Assay (Biotrak activity assay RPN2634): Equal amounts of protein form tPVAT was loaded into the 96-well ELISA plate. The plate was incubated overnight and then washed to remove unbound protein. Subsequently, a colormetric substrate was added to the wells to measure the rate of substrate breakdown (increase in color intensity) over a 90-minute period. The rate of color intensity development was measured and normalized compared to the LZR control rate.

Aortic Stiffness via Elastic Modulus: One $3 \mathrm{~mm}$ aortic ring from per animal ( $\mathrm{n}=8$ per group) was incubated in $\mathrm{Ca}^{2+}$ free Van Breemen solution $\left(119 \mathrm{mM} \mathrm{NaCl}, 4.7 \mathrm{mM} \mathrm{KCl}, 1.17 \mathrm{MgSO}_{4} \cdot 7 \mathrm{H}_{2} \mathrm{O}\right.$, $20 \mathrm{mM} \mathrm{MgCl}{ }_{2} \bullet 6 \mathrm{H}_{2} \mathrm{O}, 1.18 \mathrm{mM} \mathrm{NaH}_{2} \mathrm{PO}_{4}, 24 \mathrm{mM} \mathrm{NaHCO}_{3}, 0.03 \mathrm{mM}$ EDTA, 2mM ethylene glycol-bis ( $\beta$-aminoethyl ether)-N,N,N',N'-tetraacetic acid (EGTA), $5.5 \mathrm{mM}$ glucose) to elicit a 
passive state. The rings were then mounted on an automated motorized force transducer (Aurora Scientific Inc. model $6350 * 358$ ) and force output was recorded in lab chart software by powerlab (AD instruments). Rings were pre-conditioned by an initial 1-minute stretch eliciting $20 \mathrm{mN}$ of force and then all the tension was removed (i.e. to a force of $0 \mathrm{mN}$ ). Rings were then stretched to $10 \mathrm{mN}$ of force for 3 minutes during which time the internal diameter and wall thickness were measured. Subsequently, the automated force transducer increased the aortic ring diameter by $25 \%$ of initial internal diameter every 3 minutes until mechanical failure (determined by a dropin force following a stretch). Stress and strain equations will be modified from those previously used $^{2}$ to match those used in large arteries ${ }^{3}$ and the slope of the relationship plotted to determine the elastic modulus. Stress and strain were calculated as follows: one-dimensional stress $(\mathrm{t})=\lambda \mathrm{L} / 2 \mathrm{HD}$.

$$
\operatorname{strain}(\lambda)=(\Delta \mathrm{d} / \mathrm{d}(\mathrm{i}) .
$$

$\Delta \mathrm{d}=$ change in diameter, $\mathrm{d}(\mathrm{i})=$ initial diameter $) \mathrm{L}=$ one-dimensional load applied, $\mathrm{H}=$ wall thickness, and $\mathrm{D}=$ length of vessel.

PVAT Culture Studies: To determine the direct impact of PVAT on mechanical stiffness, LZR aortic rings ( $\mathrm{n}=4$ per treatment) were cultured for 72 hours under the following conditions; control (just media), LZR PVAT, OZR PVAT, or OZR PVAT+TNF $\alpha$ AB. Aortic rings and tPVAT segments were cultured in RMPI +GlutaMAX ${ }^{\mathrm{TM}}+25 \mathrm{mM}$ HEPES media (gibco® by life techonologies ${ }^{\mathrm{TM}}$ ) supplemented with streptomycin and kept in a $\mathrm{CO}_{2}$ cell incubator at $37^{\circ} \mathrm{C}$ under $5 \% \mathrm{CO}_{2}$. Media was replenished every 24 hours. Following the 72 hours of culture aortic rings were washed and allowed to equilibrate in $\mathrm{Ca}^{2+}$ free Van Breemen solution. Aortic rings were then subjected to the protocol described above to generate an elastic modulus. 
Figure 2.1. 200mg Verification

Figure $1200 \mathrm{mg}$ Verification

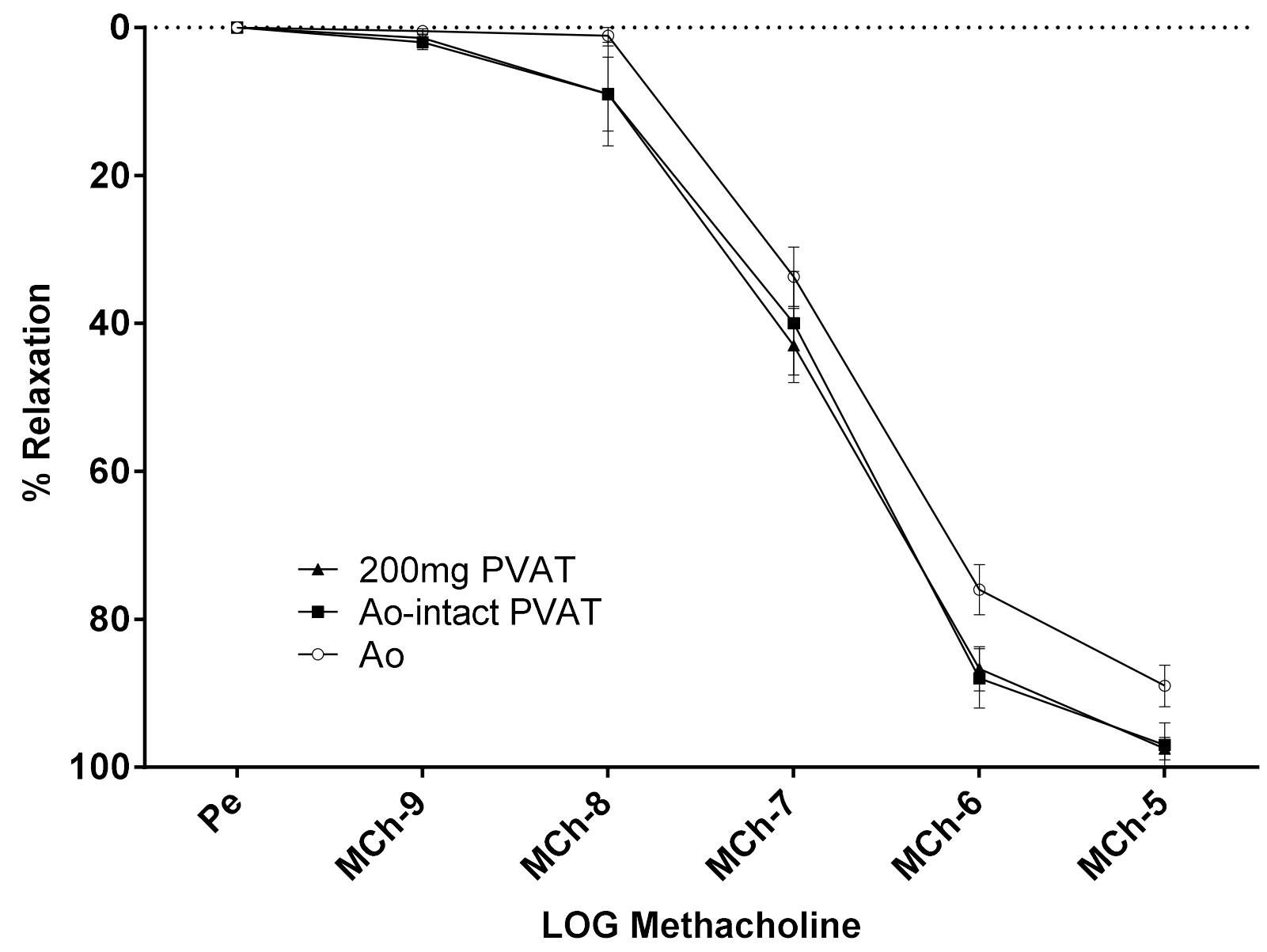

Comparison between 30-minute incubation with 200mg tPVAT to Ao with intact tPVAT (n=3).

Shows incubation with 200mg tPVAT closely represents intact tPVAT. 
Figure 2.2. Other PVAT Samples

Figure 2 Other PVAT samples

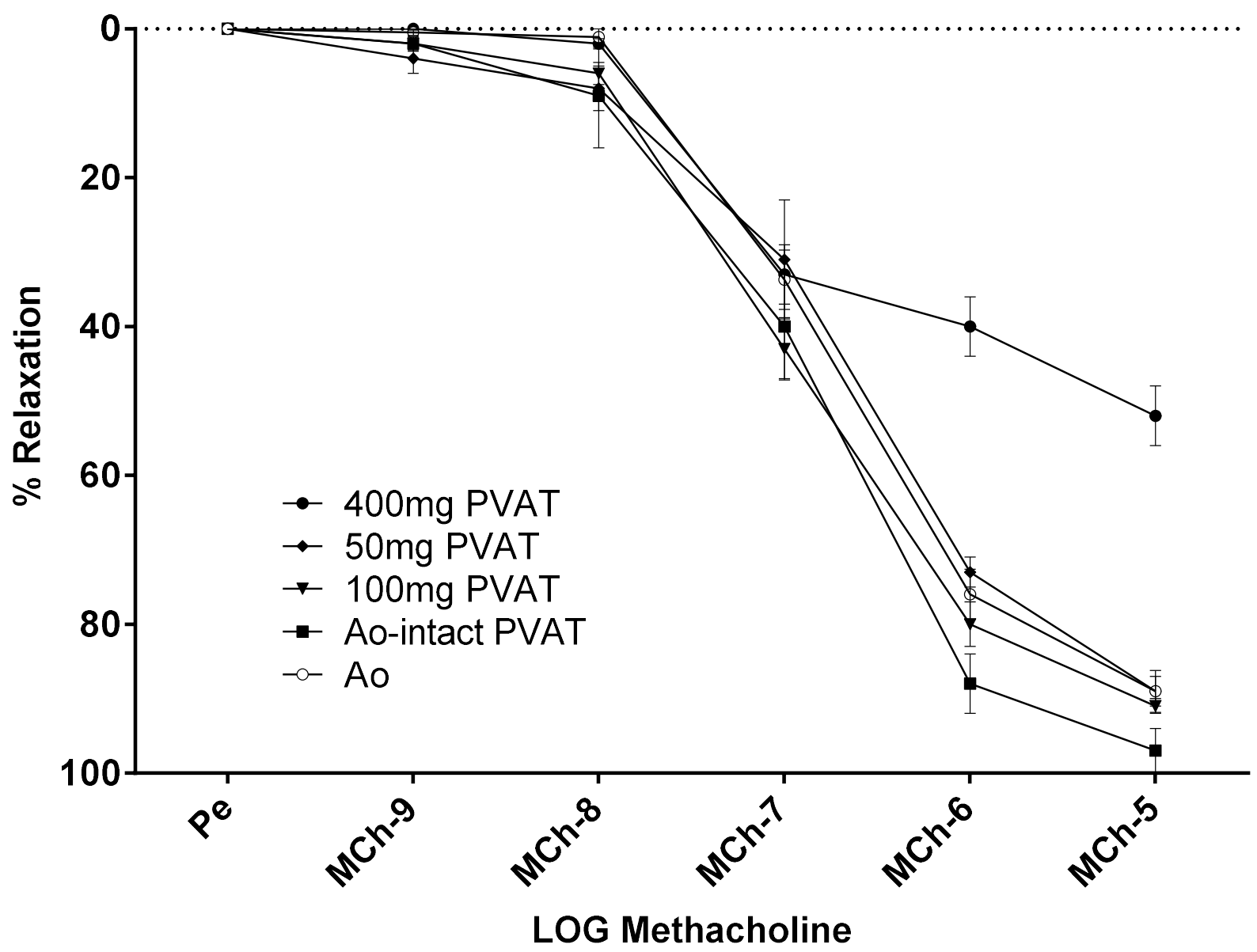

Comparison between 30-minute incubation with tPVAT to Ao with intact tPVAT ( $\mathrm{n}=3$ ). Shows these masses of tPVAT don't represent intact tPVAT. 
Figure 3 Exudate Verification

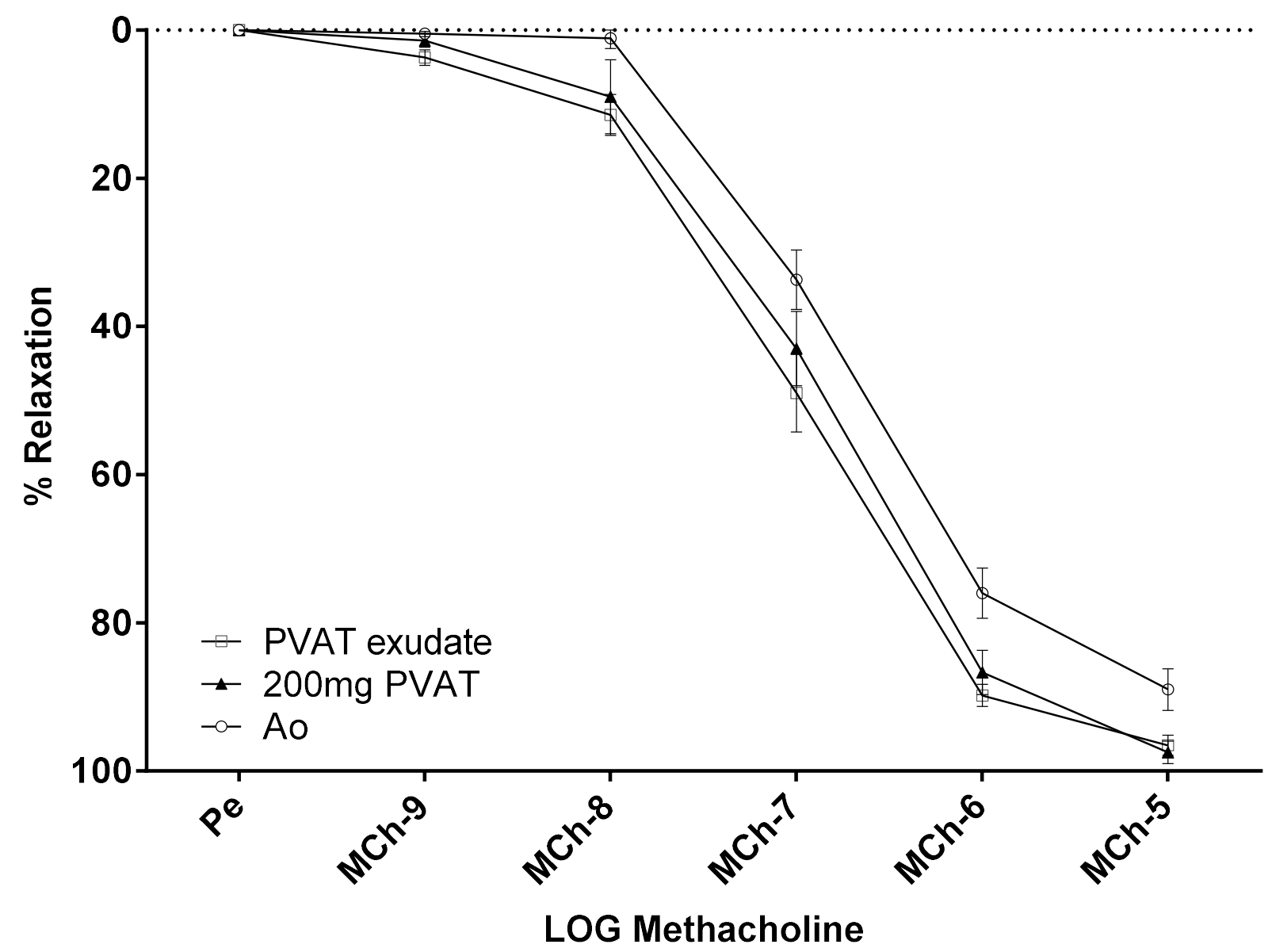

Comparison between 30-minute incubation with tPVAT or tPVAT exudate made from $200 \mathrm{mg} / \mathrm{ml}$ in HEPES buffer $(\mathrm{n}=3)$. Data shows use of exudate and 200mg of tPVAT elicit equivalent responses. 


\section{Chapter 3: Aortic Dysfunction in Metabolic Syndrome Mediated by Perivascular Adipose Tissue TNF $\alpha$ and NOX2 Dependent Pathway}

Evan DeVallance ${ }^{1}$, Kayla W Branyan ${ }^{1}$, Kent Lemaster ${ }^{2}$, I. Mark Olfert ${ }^{1}$, David M. Smith ${ }^{3}$, Emidio E Pistilli ${ }^{1}$, Jefferson C Frisbee ${ }^{2,4}$, Paul D. Chantler ${ }^{1}$

${ }^{1}$ Division of Exercise Physiology, West Virginia University School of Medicine, Morgantown, WV; ${ }^{2}$ Department of Physiology and Pharmacology, Schulich School of Medicine and Dentistry, University of Western Ontario, London, ON, Canada; ${ }^{3}$ Department of Biochemistry, West Virginia University School of Medicine, Morgantown, WV; ${ }^{4}$ Department of Medical Biophysics, Schulich School of Medicine and Dentistry, University of Western Ontario, London, ON, Canada

Running title MetS tPVAT and aortic dysfunction

Corresponding author

Paul D Chantler,

One Medical Center Drive, Morgantown, WV, 26505,

pchantler@hsc.wvu.edu,

tel: $\quad$ (304) 293-0646

fax: (304) 293-7105

Keywords: Perivascular adipose tissue, metabolic syndrome, inflammation, NOX2, proteasome, tumor necrosis factor alpha, aortic stiffness.

Word count: 8636

Total number of figures and tables: 6 Figures 6 supplemental figures 


\section{New and Noteworthy:}

- Buildup of ubiquitin due to diminishing proteasome activity is a potential mechanism of tPVAT dysfunction and pro-inflammatory cytokine production in MetS.

- tPVAT release of TNF $\alpha$ causes tPVAT ROS production through activation of NOX2 dependent pathway, activates aortic ROS production, and mediates aortic stiffness potentially through MMP-9 activity. 
$\underline{\text { Abstract: }}$ Aims: Perivascular adipose tissue (PVAT) is recognized for its vaso-active effects, however it's unclear how Metabolic Syndrome impact thoracic-aorta (t)PVAT and the subsequent effect on functional and structural aortic stiffness. Methods \& Results: Thoracic aorta and tPVAT were removed from 16-17week old lean (LZR, n=16) and obese Zucker (OZR, $\mathrm{n}=16$ ) rats. OZR presented with aortic endothelial dysfunction, assessed by wire-myography, and increased aortic stiffness, assessed by elastic modulus. OZR-tPVAT exudate further exacerbated the endothelial dysfunction reducing nitric oxide and endothelial dependent relaxation $(\mathrm{p}<0.05)$. Additionally, OZR-tPVAT exudate had increased MMP9 activity $(\mathrm{p}<0.05)$ and further increased elastic modulus of the aorta following 72-hours of coculture $(p<0.05)$. We found the observed aortic dysfunction caused by OZR-tPVAT was mediated through increased production and release of TNFa through a NADPH-oxidase 2 (NOX2) dependent pathway. We found OZRtPVAT had increased gene expression of immune markers, inflammatory cytokines, and oxidative enzymes. This coincided with increased ROS production $(\mathrm{p}<0.01)$, reduced activity of the 20S proteasome's active sites $(\mathrm{p}<0.05)$, and reduced superoxide dismutase activity $(\mathrm{p}<0.01)$. OZR-tPVAT dysfunction caused increased release of inflammatory cytokines especially TNF $\alpha$ $(\mathrm{p}<0.01)$. The greater release of pro-inflammatory cytokines from OZR-tPVAT resulted in activation of ROS in aortic rings $(\mathrm{p}<0.01)$, leading to reduced nitric oxide $(\mathrm{p}<0.05)$. OZR-tPVAT dysfunction and subsequent aortic dysfunction was inhibited by TNFa neutralization and inhibition of NOX2. Conclusion: Metabolic syndrome causes tPVAT dysfunction through a cyclical interplay between TNF $\alpha$ and NOX2 leading to tPVAT mediated aortic stiffness by activation of aortic ROS and increased MMP9 activity. 


\section{$\underline{\text { Introduction }}$}

A clustering of cardiovascular risk factors, known as metabolic syndrome (MetS), is associated with increases in oxidative stress and inflammation leading to vascular dysfunction as reported by our group and others $(3,10,24)$. In MetS, the aorta becomes less compliant through an increase in both functional and structural stiffness. Functional stiffness arises from reduced nitric oxide (NO) bioavailability increasing smooth muscle tone and redistributing circumferential stress onto the extracellular matrix (ECM) (52). The remodeling of the ECM, fragmentation of elastin and deposition of collagen, is the hallmark of structural stiffness $(13,26)$. The resulting loss of aortic compliance increases afterload on the heart (34), reduces coronary perfusion (26), and increases pulsatile flow to the periphery (28), which can ultimately lead to end organ damage (39).

Perivascular adipose tissue (PVAT) surrounds much of the vasculature and has various phenotypes and function depending on location. Over the past decade, PVAT has been shown to actively regulate vascular function. Unlike other PVAT depots, "brown-like" thoracic (t)PVAT encases the aorta and releases vasoactive factors, which promote beneficial vascular effects through the promotion of NO production (15). In disease states, PVAT becomes dysfunctional and shifts towards a pro-oxidative and pro-inflammatory state $(6,32)$. Of particular interest is the cytokine tumor necrosis factor alpha (TNF $\alpha)$, which has a potent vasoactive effect, induces the production of inflammatory cytokines, including its own transcription $(7,36)$, and activates the production of ROS from oxidative enzymes, such as NADPH oxidase $(7,25)$. Data from peripheral PVAT depots highlight the detrimental impact of a pro-oxidative and proinflammatory state on small artery function $(18,49)$, especially endothelial dependent dilation (EDD). Studies show pathological ECM remodeling and arterial stiffness is mediated through 
reductions in $\mathrm{NO}$ and $\operatorname{EDD}(13,20,23)$. These previous studies highlight the effects of individual disease states, but the effect of the concurrent risk factors in MetS on tPVAT function is limited. Additionally, much of the current MetS literature on PVAT function is in depots surrounding mesenteric or small arterials. MetS effects on tPVAT and its regulation of aortic function is poorly understood and key to understanding the vascular consequences of MetS and developing effective therapeutics.

Significant aortic dysfunction has been previously shown in a rat model of MetS the obese Zucker rat (33). However, it remains unknown to what extent tPVAT affects the pathological changes in MetS and how it regulates aortic function. In our study, we aim to uncover key pathways, which drive tPVAT dysfunction and the subsequent mediators of aortic impairment in MetS. We hypothesize phenotypic shifts in MetS PVAT leads to an increase in ROS production from TNF $\alpha$ activation of NOX2. We further hypothesize tPVAT derived pro-inflammatory cytokines activate ROS production in the aorta diminishing NO bioavailability with subsequent aortic dysfunction.

\section{$\underline{\text { Methods \& Materials }}$}

Animals: Male lean (LZR) and obese (OZR) Zucker rats were purchased from Envigo Laboratories at $8-9$ weeks of age ( $\mathrm{n}=16 /$ group). Animals were housed at the West Virginia University Health Science Center (WVUHSC) animal care facility on an approved protocol by the WVUHSC Animal Care and Use Committee, which meets the NIH guidelines for care and use of laboratory animals. Animals received standard chow and tap water ad libitum. At time of 
terminal procedures 16-17 weeks old animals were weighed (LZR $419 \pm 17 \mathrm{~g}$ vs. OZR $604 \pm 10 \mathrm{~g}$ ) then deeply anesthetized with pentobarbital sodium $(50 \mathrm{mg} / \mathrm{kg})$ and tracheal was intubated to aid in keeping a patent airway. All rats then received carotid artery and jugular vein cannulation to measure mean arterial pressure (MAP, LZR $106 \pm 2 \mathrm{mmHg}$ vs. OZR $135 \pm 6 \mathrm{mmHg}$ ) and to administer heparin, respectfully. The diaphragm was then cut and the aorta was removed and placed in ice cold Krebs Henseleit Buffer $\left(1.18 \mathrm{mM} \mathrm{KH}_{2} \mathrm{PO}_{4}, 1.2 \mathrm{mM} \mathrm{MgSO}{ }_{4} \cdot 7 \mathrm{H}_{2} \mathrm{O}, 4.7 \mathrm{mM}\right.$ $\mathrm{KCl}, 25 \mathrm{mM} \mathrm{NaHCO}$, $118 \mathrm{mM} \mathrm{NaCl}, 5.5 \mathrm{mM}$ glucose, $0.026 \mathrm{mM}$ Ethylenediaminetetraacetic acid (EDTA), $2.5 \mathrm{mM} \mathrm{CaCl}_{2} \cdot 2 \mathrm{H}_{2} \mathrm{O}$, bubbled with $95 \% \mathrm{O}_{2}$ ). tPVAT was then carefully removed from the aorta under a dissecting microscope, and the aorta was cut into $3 \mathrm{~mm}$ rings. Gene expression, inflammatory mediators were assessed in tPVAT.

Gene Expression: 50mg sections of tPVAT were incubated at $37^{\circ} \mathrm{C}$ in physiological HEPES buffer $\left(43.7 \mathrm{mM} \mathrm{NaCl}, 80 \mathrm{mM} \mathrm{KCl}, 1.17 \mathrm{mM} \mathrm{MgSO}{ }^{\bullet} \cdot 7 \mathrm{H}_{2} \mathrm{O}, 1.6 \mathrm{mM} \mathrm{NaH}_{2} \mathrm{PO}_{4}, 18 \mathrm{mM}\right.$ $\mathrm{NaHCO}_{3}, 0.03 \mathrm{mM}$ EDTA, $5.5 \mathrm{mM}$ glucose, $5 \mathrm{mM}$ HEPES) or HEPES buffer containing $4 \mu \mathrm{M}$ TNF $\alpha$ neutralizing antibody (TNF $\alpha-n A B$, Catalog \#: AF-510-NA, R\&D systems) at a ratio of $200 \mu \mathrm{g} / \mathrm{mL}$. After 1 hour PVAT was removed and snap frozen. To assess gene expression, tPVAT was homogenized in QIAzol and processed for qPCR using the RNeasy Lipid Tissue MiniKit (Qiagen), QuantiTect reverse transcription kit (Qiagen 205313). Equal concentrations of cDNA were then loaded into the QIAgility (Qiagen), which mixed 20 $\mu \mathrm{L}$ PCR reactions with QuantiTect primer assays [Adiponectin (QT01169343), $\beta$-actin (QT00193473), catalase (QT00182700), CCL5 (QT01083614), CCR3 (QT00183925), CCR5 (QT01084034), CD4 (QT00181811), CD68 (QT00372204)，CD8a (QT00177261)，GSR (QT01083285)，IFN- $\gamma$ (QT00184982)，IL-10 (QT00177618)，IL-13 (QT00184842)，IL-1ß (QT00181657)，IL-4 (QT01590316), Keap1 
(QT00189595), MMP2 (QT00996254), MMP9 (QT00178290), Gp91phox (QT00195300), Nrf2 (QT00183617), p47phox (QT00189728), SOD-1 (QT00174888), SOD2 (QT00185444), TIMP-1 (QT00185304), TNF (QT02488178), TSP-1 (QT01300607), UCP-1 (QT00183967), Qiagen] and QuantiFast Sybr Green Master Mix (Qiagen 204056). Relative quantification was carried out by the $2^{\wedge}(\mathrm{ddCt})$ method with the reciprocal used for graphical representation of negative fold changes.

Measurement of Reactive Oxygen Species: Dihydroethidium (DHE, Invitrogen D1168) assays were performed on unfixed aortic rings and tPVAT sections to evaluate ROS production. Aortic

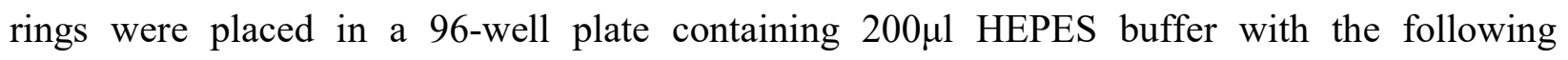
treatments: control (no drug), tPVAT, Crossover tPVAT (i.e., LZR-tPVAT on OZR aortic rings

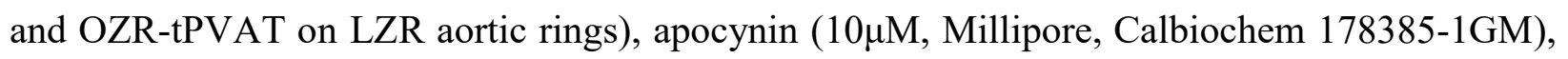
NOX2ds-TAT $(50 \mu \mathrm{M}$, Applied Biosystem Inc), or 4-Hydroxy-TEMPO (TEMPOL 100 $\mu \mathrm{M}$, Sigma-Aldrich 176141$)$ at $37^{\circ} \mathrm{C}$. Additionally, tPVAT was pretreated, with Pyrogallol $(10 \mu \mathrm{M}$, MP Biomedical 151993), NOX2ds-TAT $(50 \mu \mathrm{M})$, or TNF $\alpha-n A B(4 \mu \mathrm{M})$. Aortic rings were incubated in drug treatment for 30 minutes followed by addition of DHE to $10 \mu \mathrm{M}$ and incubated for another 30 minutes. Following completion of DHE incubation samples were washed in HEPES buffer, placed in Optimal Cutting Temperature compound (OCT, Fisher Healthcare ${ }^{\mathrm{TM}}$ ) and frozen in liquid nitrogen cooled isopentane and stored at $-80^{\circ} \mathrm{C}$. DHE OCT blocks were cut into $8 \mu \mathrm{m}$ slices using a cryostat and transferred to charged slides (Fisherbrand ${ }^{\circledR}$ Superfrost ${ }^{\circledR}$ ) and stained/mounted with DAPI mounting media (Vector laboratories). Slides were imaged with an EVOS fluorescent microscope (Invitrogen EVOS FL Auto Cell Imaging System), three images per treatment, were analyzed by ImageJ as fluorescent density/nucleus, the mean of the 3 
images/treatment were used as the mean for each animal. Values were normalized to signal from tempol treatment to eliminate background signal.

NO Bioavailability: Aortic NO production was measured according to manufacturer's instructions by 4-Amino-5-Methylamino-2', $7^{\prime}$-Difluorofluorescein-Diacetate (DAF-FM-DA Invitrogen). $3 \mathrm{~mm}$ aortic rings were placed in individual wells of a 96 -well plate containing $200 \mu \mathrm{l}$

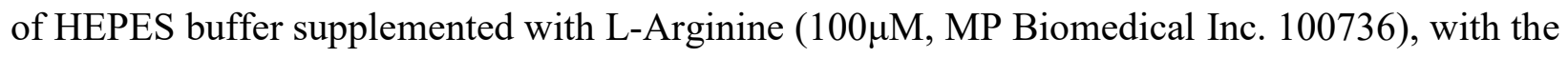
following treatments: control (no added drug), tPVAT, Crossover tPVAT, tPVAT+TNF $\alpha-n A B$ $(4 \mu \mathrm{M})$ or nitro-L-argininemethylester (L-NAME, an inhibitor of NO synthase, Sigma-Aldrich N5751). After 30-minutes of incubation with treatment, $10 \mu \mathrm{M}$ DAF-FM-DA was added and the vessel was stimulated with methacholine (MCh, 1 X10-6 Sigma-Aldrich A2251). After 10 minutes, the rings were removed, and conditioned solution was read in a plate reader (BioTek Synergy HT) excitation/emission at $495 / 515 \mathrm{~nm}$ wavelength. Fluorescence was normalized to aorta length and L-NAME value.

PVAT Cytokine Profile: tPVAT $(200 \mathrm{mg} / \mathrm{mL})$ was incubated in HEPES buffer for 2 hours at $37^{\circ} \mathrm{C}$. The tPVAT was removed and the media was snap frozen and stored at $-80^{\circ} \mathrm{C}$. The conditioned media was run on rat inflammatory panel-2 (Mesoscale discovery V-plex K15059D2), MMP9 activity ELISA (GE Biotrak activity assay RPN2634), and high molecular weight adiponectin ELISA (Mybiosource MBS020496). Additionally, tPVAT homogenates were run on inflammation rat panel-1 (Mesoscale discovery K15179C-9), 20S proteasome ELISA (Mybiosource MBS730715), and Ubiquitin ELISA (Mybiosource MBS039103). All assays were run per manufacturer's instructions. 
PVAT Tissue Function: tPVAT homogenates were run per manufacturer's instructions for SOD activity (Sigma-Aldrich 19160-1KT-F), total and phosphorylated NF-kBp65 (ThermoFisher multispecies InstantOne ${ }^{\mathrm{TM}}$ ELISA Kit, 85-86083-11). tPVAT was homogenized in HEPES buffer containing $1 \mathrm{mM}$ DTT, $2 \mathrm{mM}$ ATP, and $10 \mathrm{mM}$ magnesium chloride were assayed with

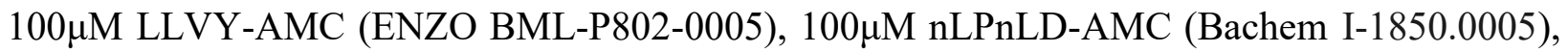
and 10 $\mu$ M RLR-AMC (Boston Biochem S-290). These substrates are cleaved by the three different protease sites in the $20 \mathrm{~S}$ core particle, and are thus a good general indicator of proteasome degradation capacity in the cell. Proteasome assays were measured by kinetic read (1 read/minute for 120 minutes) on a plate reader (ex/em $380 / 460 \mathrm{~nm}$ ) warmed to $37^{\circ} \mathrm{C}$. V-max for each assay was determined from 30 points on the linear portion of the kinetic read and normalized to V-max in the presence of the 20S inhibitor MG132.

Aortic Reactivity: $3 \mathrm{~mm}$ thoracic aortic rings (cleaned of surrounding tissue, $\mathrm{n}=14-16$ ) were rinsed in physiological salt solution and mounted in a myobath chamber between a fixed point and a force transducer (World Precision Instruments) and pre-stretched to equilibrate for 1 hour in Krebs Henseleit Buffer aerated with $95 \% \mathrm{O}_{2}$ and $5 \% \mathrm{CO}_{2}$ at $37^{\circ} \mathrm{C}$. After equilibration, aortic baseline tension was adjusted to 1 gram and vessel viability was checked with $50 \mathrm{mM}$ of $\mathrm{KCl}$ and rings not generating a rapid response were excluded from the study. To test EDD, aortic rings were pre-constricted with phenylephrine $\left(\mathrm{PE}, 1 \mathrm{X} 10^{-7} \mathrm{M}\right.$ Sigma-Aldrich $\left.\mathrm{P} 6126\right)$ and a stable tension was reached and recorded followed by increasing doses of $\mathrm{MCh}\left(1 \times 10^{-9} \mathrm{M}-1 \times 10^{-5} \mathrm{M}\right)$. Relaxation was calculated as \%relaxation for each dose of $\mathrm{MCh}$ from the following equation:

$$
\% \text { relaxation }=\left(\frac{Z-x}{Z-y}\right) \times 100 \text {, }
$$


where $\mathrm{z}=$ tension after $\mathrm{PE} 1 \mathrm{X} 10^{-7} \mathrm{M}, \mathrm{x}=$ tension following a given does of $\mathrm{MCh}$, and $\mathrm{y}=$ baseline tension.

Following the relaxation curve, the system was washed again with buffer and allowed to return to baseline. To test the effect of tPVAT on EDD, tPVAT exudate and exudate generated after treatment with $\mathrm{TNF} \alpha-\mathrm{nAB}$ and/or NOX2ds-TAT was either snap frozen and used in crossover experiments or used immediately. Exudate was added to the bath and rings incubated for 30 minutes. Pilot studies showed no difference in EDD between exudate vs. tPVAT tissue incubation. Following the incubation, relaxation curves were determines as described above. Finally, aortic rings both with and without tPVAT incubation were pre-constricted with PE $1 \mathrm{X} 10^{-7} \mathrm{M}$ and treated with increasing doses of sodium nitroprusside (SNP; $1 \times 10^{-9} \mathrm{M}-1 \times 10^{-5} \mathrm{M}$, MP Biomedical 152061).

Aortic Stiffness via Elastic Modulus: Aortic rings were incubated in $\mathrm{Ca}^{2+}$ free Van Breemen solution $\left(119 \mathrm{mM} \mathrm{NaCl}, 4.7 \mathrm{mM} \mathrm{KCl}, 1.17 \mathrm{MgSO}_{4} \cdot 7 \mathrm{H}_{2} \mathrm{O}, 20 \mathrm{mM} \mathrm{MgCl}{ }_{2} \cdot 6 \mathrm{H}_{2} \mathrm{O}, 1.18 \mathrm{mM}\right.$ $\mathrm{NaH}_{2} \mathrm{PO}_{4}, 24 \mathrm{mM} \mathrm{NaHCO} 3,0.03 \mathrm{mM}$ EDTA, $2 \mathrm{mM}$ ethylene glycol-bis ( $\beta$-aminoethyl ether)$\mathrm{N}, \mathrm{N}, \mathrm{N}^{\prime}, \mathrm{N}^{\prime}$-tetraacetic acid (EGTA), $5.5 \mathrm{mM}$ glucose) to elicit a passive state and mounted on an automated motorized force transducer (Aurora Scientific Inc. model-6350*358) and force output was recorded in lab chart software by powerlab (AD instruments). Rings were pre-conditioned by a $20 \mathrm{mN} \mathrm{1-minute} \mathrm{stretch} \mathrm{and} \mathrm{then} \mathrm{all} \mathrm{tension} \mathrm{was} \mathrm{removed} \mathrm{(i.e.} \mathrm{force}=0 \mathrm{mN}$ ). Rings were then stretched to $10 \mathrm{mN}$ of force for 3 minutes and the internal diameter and wall thickness were measured. Subsequently, the automated force transducer increased the aortic ring diameter by $25 \%$ of initial internal diameter every 3 minutes until mechanical failure. Stress and strain 
equations were modified from those previously used (3) to match those used in large arteries (14) and the slope of the relationship plotted to determine the elastic modulus. Stress and strain were calculated as follows:

$$
\begin{aligned}
& \text { one-dimensional stress }(\mathrm{t})=\lambda \mathrm{L} / 2 \mathrm{HD} \text {. } \\
& \operatorname{strain}(\lambda)=(\Delta \mathrm{d} / \mathrm{d}(\mathrm{i})
\end{aligned}
$$

$\Delta \mathrm{d}=$ change in diameter, $\mathrm{d}(\mathrm{i})=$ initial diameter $) \mathrm{L}=$ one-dimensional load applied, $\mathrm{H}=$ wall thickness, and $\mathrm{D}=$ length of vessel.

PVAT Culture Studies: To determine the direct impact of PVAT on mechanical stiffness, LZR aortic rings ( $\mathrm{n}=4 /$ treatment) were cultured for 72 -hours in RMPI + GlutaMAXTM $+25 \mathrm{mM}$ HEPES media (gibco ${ }^{\circledR}$ by Life Techonologies ${ }^{\mathrm{TM}}$ ) with streptomycin and kept in an incubator at $37^{\circ} \mathrm{C}$ and $5 \% \mathrm{CO}_{2}$, under the following conditions; control (just media), LZR PVAT, OZR PVAT, or OZR PVAT+TNF $\alpha$-nAB. Media was discarded and replenished daily. Following the 72-hours of culture, the aortic rings were subjected to the protocol described above to generate an elastic modulus.

$\underline{\text { Statistics: }}$ All data are represented as means \pm standard error. All experiments were run in duplicate, and the reads were averaged for each animal. Data analysis and graphing were conducted using GraphPad Prism 6 software and $\mathrm{p}<0.05$ was set as the mark for statistical significance. Comparisons between LZR and OZR were conducted using a simple student T-test, while repeated measure ANOVA analysis was used for treatment effects, effect of tPVAT, and aortic ring relaxation. 


\section{$\underline{\text { Results }}$}

\section{tPVAT Environment}

Gene Expression: To assess the effect of MetS on the tPVAT environment we first compared gene expression of key transcripts involved in adipose phenotype, pro/anti- inflammatory cytokines, and pro \& anti-oxidants. OZR showed a significant drop in uncoupling protein-1 (UCP-1) expression suggesting a shift towards a "whiter" phenotype $(\mathrm{p}<0.01$, Fig.3.1A) and increased immune cell markers CD68 (macrophages, $\mathrm{p}<0.05$ ) and CD8a (cytotoxic T-cells, $\mathrm{p}<0.05$ ) (Fig.3.1A). This was accompanied by an increased expression of proinflammatory/oxidative genes (Fig.3.1B). We also identified a reduction in gene expression of the protective anti-inflammatory/oxidant genes, which could exacerbate the proinflammatory/oxidative changes of tPVAT from OZR's (Fig.3.1C). With the observed increase in oxidative gene expression we sought to measure ROS production and SOD activity in tPVAT.

tPVAT Function: ROS was significantly increased in OZR-tPVAT compared to LZR-tPVAT $(\mathrm{p}<0.001$, Fig.3.2A \& ImageS1), in part, due to a reduced SOD activity in OZR-tPVAT $(\mathrm{p}<0.001$, Fig.3.2B). Under normal physiological conditions oxidized cellular components are cleared by the proteasome system. With the sizable increase in ROS production we were interested in the clearance of oxidized cellular components. We found proteasome activity was globally diminished in OZR-tPVAT. This was shown by reduced $(\mathrm{p}<0.01)$ V-max across all 3 assays: LLVY, RLR, and nLDnLP (chymotrypsin-like, trypsin-like, and peptidylglutamylpeptide hydrolyzing active sites) (Fig.3.2C). This proteasome dysfunction was again highlighted 
by an increased expression of ubiquitin, suggesting a reduced ability to clear ubiquitinated proteins (Fig.3.2D), which is linked to increased inflammation.

Proteasome dysfunction and buildup of ubiquitin are linked to increases in inflammation, thus we evaluated tPVAT cytokine production. First, we looked at chemoattractant cytokines because of the increased immune specific gene expression. Cytokine assays showed an increase $(\mathrm{p}<0.05)$ in both monocyte chemoattractant protein-1 (MCP-1) and chemokine (C-X-C motif) ligand-1 (KC/Gro) (Fig.3.3A). Further, all cytokines profiled showed significant alterations in OZR compared to LZR, with pro-inflammatory mediators (TNF $\alpha$, IL-1 $\beta$, IL-6, IFN- $\gamma$, and TSP-1) significantly elevated and anti-inflammatory mediators (IL-4, IL-5, IL-10, IL-13, and adiponectin) significantly reduced (Fig.3.3A\&B). These data demonstrated altered tPVAT function in MetS, we then wanted to investigate how tPVAT in OZR affected aortic ROS.

tPVAT Mediated Aortic ROS Production: ROS production was higher in OZR aorta compared to LZR ( $<<0.05$, Fig.3.4A \& Supplemental image2). We then incubated the LZR aorta with LZR tPVAT and identified a slight non-significant reduction in ROS production. In contrast, when the OZR aorta was incubated with OZR tPVAT, aortic ROS production was increased by $\sim 120 \%$ $(\mathrm{p}<0.001$, Fig.3.4A \& Supplemental image2). Further, the activation of aortic ROS production was significantly inhibited when the OZR aorta was treated with apocynin, and to a lesser extent with NOX2ds-TAT (Fig.3.4A \& Supplemental image2). The SOD activity in the OZR aorta was diminished compared to LZR ( $<<0.001$, Fig.3.4B). The increased ROS production has the potential to impact NO production and EDD. 
tPVAT Effect on Nitric Oxide and Aortic Relaxation: As expected, we found reduced NO bioavailability in the aorta from OZR compared to LZR (Fig.3.4C). Adding to this known finding, we showed aortic NO production was further reduced by $\sim 20 \%(\mathrm{p}<0.01)$ in the presence of OZR-tPVAT (Fig.3.4C). In contrast, LZR-tPVAT increased $(\mathrm{p}<0.05)$ aortic NO production in the LZR by $\sim 15 \%$ (Fig.3.4C). The aortic EDD (without the presence of tPVAT) in OZR was blunted by $\sim 15 \%$ in comparison to LZR EDD (p<0.01, Fig.3.4D). In the OZR, tPVAT further blunted aortic EDD by $\sim 10 \%(\mathrm{p}<0.01)$ compared to OZR without tPVAT (Fig.3.4C). In contrast, LZR aortic EDD in the presence of LZR-tPVAT improved EDD by $5 \%(\mathrm{p}<0.05$, Fig.3.4D). Importantly, endothelial independent dilation to SNP was not different between the two groups in the presence or absence of tPVAT ( $\mathrm{p}>0.05$, maximal relaxation: LZR Ao $101 \pm 0.9 \%$, LZR tPVAT-Ao $100 \pm 2.1 \%$, OZR Ao 98 $\pm 1.5 \%$, OZR tPVAT-Ao $97 \pm 4.8 \%$ ). These data illustrated tPVAT caused further impairment in OZR aortic EDD. We then wanted to determine if this was due to the release of cytokines from tPVAT or, in part, due to intrinsic properties of the OZR aorta. To assess this, we preformed crossover experiments were the LZR aorta was exposed to OZR-tPVAT media and vice versa.

PVAT Crossover Treatments: First we explored the effect of crossover tPVAT treatment on aortic ROS production. Interestingly, the LZR "health" aorta was not protected against OZRtPVAT activation of aortic ROS production ( $\mathrm{p}<0.001$, Fig.3.4E). Additionally, LZR-tPVAT was treated with pyrogallol to produce ROS. LZR-tPVAT with pyrogallol increased ROS production (Fig.3.4E \& Supplemental image3) and decreased EDD from the LZR aorta $(\mathrm{p}<0.05$, max relaxation: LZR tPVAT+ pyrogallol-Ao $74 \pm 2.6 \%$ vs LZR tPVAT-Ao $87 \pm 1.8 \%$ ) but not to the same extent as OZR-tPVAT ( $\mathrm{p}<0.05$, max relaxation: LZR tPVAT+ pyrogallol-Ao $74 \pm 2.6 \%$ vs 
OZR tPVAT- LZR Ao $64 \pm 1.5 \%$ ). We then incubated LZR-tPVAT with the OZR aorta, which reduced aortic ROS production ( $\mathrm{p}<0.01$, Fig.3.4E). Whereas, when OZR-tPVAT was incubated with the LZR aorta NO production was reduced, with subsequently impaired aortic EDD $(p<0.01)$ in the LZR (Fig.3.4F\&G). Conversely, the crossover treatment improved $(p<0.01)$ NO production and aortic EDD in OZR treated with LZR-tPVAT (Fig.3.4F\&G). After observing a $\sim 10$-fold increase in TNF gene expression and $\sim 10 \mathrm{X}$ increase in TNF $\alpha$ secretion in OZR tPVAT, we repeated the above experiments with a $\mathrm{TNF} \alpha-\mathrm{nAB}$ to determine its role in orchestrating the observed dysfunction. Additionally, we targeted NOX2 the prominent NOX enzyme of inflammatory immune cells, as it is a known target of TNF $\alpha$.

\section{PVAT with TNFa Neutralization}

Gene Expression: We first sought to determine the role of TNF $\alpha$ on OZR-tPVAT gene expression. $\mathrm{TNF} \alpha-\mathrm{nAB}$ treatment (ex-vivo) significantly down-regulated phosphorylation of nuclear factor kappa-light-chain-enhancer (NF-kB, Fig.3.5A), reduced TNF inflammatory gene expression, and decreased gene expression of NOX2 and its regulator p47phox (Fig.3.5B). Additionally, TNF $\alpha-n A B$ reduced $(p<0.05)$ MMP-9 gene expression and increased $(p<0.05)$ the gene expression for Nrf2, IL-10, and adiponectin (Fig.3.5B), highlighting the role TNF $\alpha$ in the regulation of gene expression in the tPVAT. However, does TNF $\alpha-n A B$ treatment ameliorate tPVAT ROS production and the activation of aortic ROS by tPVAT?

Reactive Oxygen Species: TNF $\alpha-n A B$ treatment caused a marked reduction $(\mathrm{p}<0.001)$ in the DHE signal from OZR-tPVAT to similar levels observed with inhibition by NOX2ds-TAT (Fig. 3.5C \& Supplemental image4). In turn, the TNF $\alpha-n A B$ treatment in OZR-tPVAT completely 
inhibited the tPVAT activation of ROS in both OZR and LZR aorta $(\mathrm{p}<0.001$, Fig.3.5C \& Supplemental image4). Further, highlighting the importance of TNF $\alpha$ activation of NOX2 in OZR-tPVAT dysfunction, inhibition with NOX2ds-TAT completely inhibited the tPVAT activation of ROS produced by the aorta ( $<<0.001$, Fig.3.5C \& Supplemental image4). To determine if the diminished proteasome activity in OZR-tPVAT was acutely mediated by TNF $\alpha$ we cultured OZR-tPVAT with TNF $\alpha-n A B$ and ran all 3 proteasome assays. The results showed no effect of TNF $\alpha-n A B$ (VMAX: LLYV $1.2 \pm 0.05$, RLR $2.5 \pm 0.07$, nLPnLD $0.5 \pm 0.04$ $\mathrm{p}>0.05)$.

Nitric Oxide and Aortic Reactivity: With TNF $\alpha-n A B$ treatment yielding beneficial effects on gene expression and ROS production we examined its actions on NO and aortic EDD. TNF $\alpha-$ nAB treatment in OZR-tPVAT inhibited tPVAT mediated reduction of NO production from OZR and LZR aortas $(\mathrm{p}<0.001$, Fig.3.5D). In turn, TNF $\alpha-n A B$ treatment in OZR-tPVAT completely inhibited tPVAT mediated dysfunction of aortic EDD in both OZR and LZR ( $p<0.01$, Fig.3.5E\&F). To highlight the importance of NOX2 in the TNF $\alpha$ mediated aortic dysfunction, we treated OZR-tPVAT with NOX2ds-TAT and demonstrated aortic EDD was completely restored ( $\mathrm{p}<0.001$, Fig.3.5E), however the combination of TNF $\alpha-\mathrm{nAB}$ and NOX2ds-TAT caused no further improvement ( $\mathrm{p}>0.05$, maximal relaxation: $71 \pm 3.0 \%)$. Additionally, to show the importance of tPVAT activation of aortic ROS production, the LZR and OZR aortas were pretreated with TEMPOL, which prevented the impaired EDD $(\mathrm{p}<0.05$, maximal relaxation: OZR tPVAT-Ao+TEMPOL $78 \pm 1.8 \%$ vs OZR tPVAT-Ao $58 \pm 2.1 \%$ vs OZR Ao $68 \pm 1.8 \%$ ). Our data to this point has established the role of OZR-tPVAT mediating NO regulation of smooth 
muscle tone, and thus functional aortic stiffness. Additionally, we assessed the role of MetS tPVAT on aortic remodeling and structural stiffness.

Elastic Modulus: Aortic stiffness was increased $(\mathrm{p}<0.01)$ by $\sim 90 \%$ in the OZR compared to LZR (Fig.3.6A). Thus, we wanted to examine the role of tPVAT in OZR aortic stiffness. We first examined tPVAT gene expression and found an increased MMP9 expression $(\mathrm{p}<0.05)$ but no change in MMP2 and TIMP-1 (Fig.3.6B). We then examined the aortic protein levels of TIMP-1, which were decreased in OZR compared to the LZR (Fig.3.6C). Further, activity levels of MMP9, assessed in tPVAT exudate, were increased in OZR compared to LZR, and treating tPVAT with the TNF $\alpha$-nAB prevented the increase in MMP9 activity (Fig.3.6D). This laid the groundwork for the potential role of OZR-tPVAT to affect aortic remodeling. Next, we examined the direct contribution of tPVAT to aortic stiffness, and the role of TNF $\alpha$. Co-culturing the LZR aorta with LZR-tPVAT did not alter the elastic modulus compared to culture control; however, the LZR aorta cultured with OZR-tPVAT showed an increased $(\mathrm{p}<0.01)$ elastic modulus (Fig.3.6E), which was completely inhibited when the LZR aorta with OZR-tPVAT were cultured with the TNF $\alpha-n A B(p<0.01$, Fig.3.6E).

\section{$\underline{\text { Discussion }}$}

Previous studies have detailed the effect of hypertension and obesity on PVAT function. However, MetS pathology is dependent on the complex interactions of its components and may yield differing effects than a component in isolation. Uncovering the distinct and coordinating signaling pathways of the MetS components in tPVAT warrants future evaluation. Our present study identified key mediators of tPVAT dysfunction and demonstrated an essential role of 
tPVAT on aortic dysfunction in MetS. We identified, for first time, diminished 20S proteasome activity as a potential mechanism of tPVAT dysfunction in MetS. Additionally, we demonstrated TNF $\alpha$ as a key stimulator of tPVAT ROS production through a NOX2 dependent pathway, and activation of aortic ROS production through a non-NOX2, NADPH oxidase pathway. Additionally, we demonstrated the cyclical activation of NOX2 ROS and TNF $\alpha$ by one another was essential for the observed aortic dysfunction. Finally, we demonstrated tPVAT from OZR could directly cause aortic stiffness through a TNF $\alpha$ dependent mechanism targeting MMP9 activity. Chronic effects and the temporal development of vascular dysfunction in MetS are well defined by our group and others $(3,10,24)$. However, the role of tPVAT in orchestrating aortic function in MetS was previously unknown. Collectively our study has important implications of tPVAT pathological consequences on aortic stiffness in MetS and highlights the potential avenue of adipo-centric therapeutic development.

\section{MetS PVAT Environment}

Similar to what has been shown in obesity (45), our data showed a phenotypic "whitening" of the MetS tPVAT, supported by the change in UCP-1 expression. Given that over expressing UCP-1 can inhibit ROS production (30), the downregulation of UCP-1 in our study may suggest an avenue for the increased tPVAT ROS production noted in the OZR. Additionally, UCP-1 can be downregulated by pro-inflammatory cytokines released from immune cells, thus linking phenotypic shifts in PVAT with immune infiltration and ROS (40). In the current study the phenotypic shift in TPVAT was likely driven by increased $\mathrm{KC} /$ Gro and MCP-1 levels, resulting in a loss of anti-inflammatory cytokines and increased inflammatory immune cell markers (CD68 and CD8a), which produce $\mathrm{TNF} \alpha$ and possess NOX2. Our data from inhibition of the 
NOX p47phox subunit suggests NOX2 was largely responsible for the tPVAT ROS production. Further, an increased NOX2 activity coupled with a substantial reduction in SOD activity in OZR-tPVAT, likely accounts for the increased ROS production in tPVAT. Importantly, our data showed TNF $\alpha$ was a key mediator for the elevated ROS in MetS tPVAT. Activation of NOX2 ROS appears to be dependent on TNF $\alpha$-nAB treatment, as the simultaneous treatment with both NOX2ds-TAT and TNF $\alpha$-nAB didn't cause a further reduction in ROS production. This is in line with the literature suggesting multiple $\mathrm{TNF} \alpha$ functions are carried out by the activation of NADPH oxidases $(25,29)$. Additionally, the TNF $\alpha-n A B$ treatment showed partial restoration of tPVAT gene expression, likely due to direct action on TNF $\alpha$ and subsequent reductions in ROS (43) resulting in the observed decrease in NF- $\mathrm{KB}$ activation. This suggests chronic changes in oxidative and inflammatory machinery might also be driven by $\mathrm{TNF} \alpha$ and its activation of NOX2. Interestingly, the inhibition of NOX and/or the use of the TNF $\alpha-n A B$ did not completely inhibit ROS production, and ROS levels were still well above those measured in LZR-tPVAT. This highlights the role of other oxidative enzymes, and potentially other cytokine mediators playing some role in PVAT ROS production.

Recently, both obese human and mouse visceral adipose tissue showed reduced chymotrypsinlike activity in the proteasome and was linked to development of insulin resistance, a hallmark of MetS (8). We wanted to build upon these observations, as tPVAT is more protein dense than visceral adipose tissue, which we speculate magnifies the importance of proteasome function. ROS is known to damage and misfold proteins, which are cleared by the $26 \mathrm{~S}$ proteasome. The $26 \mathrm{~S}$ comprises of the $20 \mathrm{~S}$ core bound to one or two $19 \mathrm{~S}$ regulatory particles, which feed ubiquitinated and damaged proteins into the 20S core (46). Our results showed increased ROS, 
and for the first time in tPVAT, impaired proteasome function. The accumulation of ubiquitinated proteins is likely due to this loss of proteasome capacity, and therefore the accumulation of proteasomal substrates. Interestingly, diminished proteasome function was not due to the loss of $20 \mathrm{~S}$ proteasome levels (Fig 2) The increase accumulation of damaged and misfolded proteins can lead to cellular and oxidative stress $(17,21,35)$. Specifically, the buildup of oxidized and ubiquitin, through activation of endoplasmic reticulum stress, can induce the production of inflammatory cytokines (17). Suggesting, the observed proteasome dysfunction is an integral mechanism of increased pro-inflammatory cytokine production. Future endeavors will be aimed at assessing the causative and or exacerbating role of the entire ubiquitinproteasome system in disease mediated PVAT dysfunction.

\section{tPVAT Regulation of Aortic Function}

Our data showed a blunted aortic EDD in OZR, which was further reduced in the presence of OZR-tPVAT. The impaired EDD was derived from reduced bioavailability of NO in OZR aorta, which was further reduced $(\sim 20 \%)$ in the presence of OZR-tPVAT. Pro-inflammatory cytokines and ROS, which were increased in OZR-tPVAT, have the potential to interfere with NO bioavailability $(27,54)$.

The acute tPVAT impairment of the aorta was due to OZR-tPVAT inducing over a 100\% increase in ROS production from the already elevated levels in the OZR aorta. The production of ROS (via NOX) can interfere with NO bioavailability through the direct interaction with NO forming peroxynitrite (54), and through oxidation of the eNOS essential co-factor tetrahydrobiopterin (48). Our data implicates NOX, but not NOX2, in aortic ROS production. 
This is in opposition to Serpillon et.al. (44) who showed p47phox inhibition in the aorta caused a profound reduction of ROS. We speculate this difference may be due to the advanced diabetic state of the rats in Serpillon et.al. study (44) resulting in a key shift in oxidative enzymes upon the development of type-2 diabetes, and potentially through the influence of advanced glycation end products receptor signaling (57). Our data shows NOX2 production of ROS in tPVAT is essential in causing tPVAT activation on aortic ROS. Experimentally creating an oxidative environment with pyrogallol in healthy tPVAT was unable to recreate the same level of aortic dysfunction as MetS tPVAT. This would suggest the phenotypic changes in MetS tPVAT are essential for the production capacity of inflammatory mediators that activate aortic ROS, and interfere with NO bioavailability.

Previous data shows TNF $\alpha$ activates the production of ROS from oxidative enzymes, such as NADPH oxidase $(25,29)$. In our study, we showed the activation of aortic ROS production by OZR tPVAT could be completely abolished by a TNF $\alpha$-nAB. Similarly, albeit in a completely different vascular bed, the small resistance vessels from obese patients showed an increased gene expression of TNF in PVAT, and use of a TNF $\alpha$ receptor inhibitor reduced vessel ROS production (49), but due to experimental design they were unable to differentiate between basal and PVAT activation of ROS. In addition to direct activation of ROS TNF $\alpha$ mediates expression of IL-1 $\beta$ (47), another stimulator of oxidative enzymes (19). Additionally, IL-1 $\beta$ can act to enhance TNF $\alpha$ signaling through regulation of TNF receptors (41). This implicates IL-1 $\beta$ in a supportive role to TNF $\alpha$ in mediating OZR-tPVAT regulation of aortic dysfunction. Both TNF $\alpha$ and IL-1 $\beta$ levels can be regulated by IL-10 $(37,56)$, which we showed to be reduced in exudate from OZR-tPVAT. In addition, IL-10 is a known inhibitor of oxidative enzymes (9) suggesting 
losing IL-10 release from tPVAT removes the brakes from ROS production and exacerbates the increase of inflammatory cytokines. Finally, our data showed elevated IFN- $\gamma$, which has been implicated in PVAT mediation of vascular dysfunction. However, TNF $\alpha$-nAB treatment completely blocked OZR-tPVAT mediated aortic dysfunction and no evidence suggests a role of TNF $\alpha$ in IFN- $\gamma$ secretion. This suggests IFN- $\gamma$ secretion may not affect acute aortic function in MetS; however, it may play a role in chronic vascular dysfunction as previously shown (32).

In addition, MetS altered levels of cytokines with direct impact on NO production. TSP-1, a multifunctional homotrimeric matrix glycoprotein, was released from tPVAT at a higher concentration in OZR than LZR. Importantly, TSP-1 can inhibit eNOS activation and thus reduce NO production (22). Further, TSP-1 has a direct role in mediating immune infiltration (31), a key source of tissue inflammation. However, TSP-1 expression can also be mediated by $\mathrm{TNF} \alpha$ signaling (12) suggesting a role for TNF $\alpha$ mediating TSP-1 production as a potential mechanism of tPVAT mediated aortic dysfunction in MetS. Another potential mechanism for the reduced NO bioavailability could be the reduced release of adiponectin from OZR-tPVAT. Adiponectin can inhibit inflammation (50) and promote NO production (51). This is in concurrence with data showing PVAT derived adiponectin regulates small (100 to $150 \mu \mathrm{m}$ diameter) peripheral artery function (18). These pathways likely only play a small role as our data, in Fig.S2, showed pretreatment of the aorta with TEMPOL prevented most of the tPVAT mediated impairment. However, the chronic loss or TNF $\alpha$ inhibition of adiponectin stimulating (51) eNOS gene expression may play a part in the pathological loss of vascular eNOS. The chronic effect of tPVAT derived TNF $\alpha$ on the aorta is beyond the scope of this study and warrants future investigations. 


\section{tPVAT mediated aortic stiffness}

In addition to NO regulation of EDD, previous studies have shown NO is an essential regulator of ECM remodeling and aortic structure $(20,23)$. In obese and aged mice, tPVAT was shown to increase arterial stiffness through alterations of oxidative status, leading to elastin fragmentation $(6,14)$. However, PVAT may also directly affect ECM remodeling as adipocytes and immune cells express MMPs $(2,5)$, in particular, MMP9 which is highly associated with aortic stiffness and displays elastase activity (55). The fragmentation of elastin increases aortic stiffness by causing the loading of collagen fibers at lower pressures (26). We believe tPVAT production of $\mathrm{TNF} \alpha$ plays an important role in the aortic stiffening with MetS through a number of pathways. First, TNF $\alpha$ can stimulate the production of MMP9 directly (53) and we found that OZR-tPVAT had increased MMP9 activity, which was inhibited by TNF $\alpha$-nAB treatment. Second, TNF $\alpha$ can indirectly stimulate MMP9 though its promotion of other cytokines, whereby both IL-1 $\beta$ and TSP-1 can activate MMP9 $(4,11)$ and active MMP9 can cleave TNF $\alpha(16)$ and IL-1 $\beta$ into active forms (42). Third, ROS has also been implicated in the fragmentation of elastin $(6,14)$, thus TNF $\alpha$ activation of ROS may also play a role in the observed aortic stiffness in our study. Fourth, reduced levels of TIMP-1 (a tissue inhibitor of MMPs) can further add to MMP9 mediated aortic dysfunction (38). However, a limitation of our study was that the MMP9 activity assay was performed after the ELISA pull-down of MMP9 and bound MMP9 was activated. However, this might suggest, because of higher LZR-tPVAT TIMP-1 levels the magnitude of difference in-vivo for activity of MMP9 is even greater in OZR compared to LZR. Additionally, our co-culture experiment was limited due to the lack of intraluminal flow, which is an important 
for shear stress mediated release of NO and regulation of stiffness. However, the use of a media control helps to account for the increase in stiffness due to the lack of flow.

\section{Clinical Outlook}

The findings from the current study may help to elucidate mechanisms underlying increased aortic stiffness. Our data suggests adding treatment for adipose tissue dysfunction to a multifaceted therapeutic approach in MetS may improve vascular function. Our data identifies tPVAT localized NOX2 as an essential component of tPVAT mediated aortic dysfunction. As NOX2 is predominantly found in immune cells(1) the development of adipose tissue specific immunotherapy or tissue specific delivery of inhibitors might have potential therapeutic benefits. Lastly, building evidence suggests restoring functionality of the ubiquitin-proteasome system in MetS might restore adipose function and insulin sensitivity $(8,21)$, and our data suggest a potential pleiotropic effect on vascular function.

\section{$\underline{\text { Conclusions }}$}

In summary, we are the first to show in MetS a comprehensive picture of tPVAT TNF $\alpha$ production, which regulates gene expression and ROS production (specifically NOX2 derived ROS) in the tPVAT. Additionally, we show global reductions in proteasome function in MetS tPVAT. These effects of MetS on tPVAT increased the production of TNF $\alpha$, TSP-1, and IL-1 $\beta$ and decreased the production of IL-10 and adiponectin, which leads to a reduction in NO, EDD, MMP9 activation, and increases in structural stiffness. These data show tPVAT dysfunction is a major driving force in MetS aortic impairment and highlight the potential for adipo-centric therapeutics. 


\section{Funding}

This study was supported by the American Heart Association grants IRG14330015, pre-doctoral fellowship AHA (14PRE20380386); National Institute of General Medical Sciences of the National Institutes of Health (U54GM104942, and 5P20GM109098).

\section{$\underline{\text { Acknowledgments }}$}

We would like to acknowledge Vincent Setola $\mathrm{PhD}$ and David Siderovski $\mathrm{PhD}$ for assistance with PCR experiments and the use of their Qiagen equipment.

The authors declare no conflicts of interest 


\section{$\underline{\text { References }}$}

1. Bedard K and Krause KH. The NOX family of ROS-generating NADPH oxidases: physiology and pathophysiology. Physiological reviews 87: 245-313, 2007.

2. Bouloumie A, Sengenes C, Portolan G, Galitzky J, and Lafontan M. Adipocyte produces matrix metalloproteinases 2 and 9: involvement in adipose differentiation. Diabetes 50: 2080-2086, 2001.

3. Brooks SD, DeVallance E, d'Audiffret AC, Frisbee SJ, Tabone LE, Shrader CD, Frisbee JC, and Chantler PD. Metabolic syndrome impairs reactivity and wall mechanics of cerebral resistance arteries in obese Zucker rats. American journal of physiology Heart and circulatory physiology 309: H1846-1859, 2015.

4. Brown RD, Jones GM, Laird RE, Hudson P, and Long CS. Cytokines regulate matrix metalloproteinases and migration in cardiac fibroblasts. Biochemical and biophysical research communications 362: 200-205, 2007.

5. Chakraborti S, Mandal M, Das S, Mandal A, and Chakraborti T. Regulation of matrix metalloproteinases: an overview. Molecular and cellular biochemistry 253: 269-285, 2003.

6. Chen JY, Tsai PJ, Tai HC, Tsai RL, Chang YT, Wang MC, Chiou YW, Yeh ML, Tang MJ, Lam CF, Shiesh SC, Li YH, Tsai WC, Chou CH, Lin LJ, Wu HL, and Tsai YS. Increased aortic stiffness and attenuated lysyl oxidase activity in obesity. Arteriosclerosis, thrombosis, and vascular biology 33: 839-846, 2013.

7. Chen XL, Zhang Q, Zhao R, and Medford RM. Superoxide, H2O2, and iron are required for TNF-alpha-induced MCP-1 gene expression in endothelial cells: role of Rac1 and 
NADPH oxidase. American journal of physiology Heart and circulatory physiology 286: H10011007, 2004.

8. Diaz-Ruiz A, Guzman-Ruiz R, Moreno NR, Garcia-Rios A, Delgado-Casado N, Membrives A, Tunez I, El Bekay R, Fernandez-Real JM, Tovar S, Dieguez C, Tinahones FJ, Vazquez-Martinez R, Lopez-Miranda J, and Malagon MM. Proteasome Dysfunction Associated to Oxidative Stress and Proteotoxicity in Adipocytes Compromises Insulin Sensitivity in Human Obesity. Antioxidants \& redox signaling 23: 597-612, 2015.

9. Didion SP, Kinzenbaw DA, Schrader LI, Chu Y, and Faraci FM. Endogenous interleukin-10 inhibits angiotensin II-induced vascular dysfunction. Hypertension 54: 619-624, 2009.

10. Donley DA, Fournier SB, Reger BL, DeVallance E, Bonner DE, Olfert IM, Frisbee JC, and Chantler PD. Aerobic exercise training reduces arterial stiffness in metabolic syndrome. Journal of applied physiology 116: 1396-1404, 2014.

11. Donnini S, Morbidelli L, Taraboletti G, and Ziche M. ERK1-2 and p38 MAPK regulate MMP/TIMP balance and function in response to thrombospondin-1 fragments in the microvascular endothelium. Life sciences 74: 2975-2985, 2004.

12. Fairaq A, Goc A, Artham S, Sabbineni H, and Somanath PR. TNFalpha induces inflammatory stress response in microvascular endothelial cells via Akt- and P38 MAP kinasemediated thrombospondin-1 expression. Molecular and cellular biochemistry 406: 227-236, 2015.

13. Fitch RM, Vergona R, Sullivan ME, and Wang YX. Nitric oxide synthase inhibition increases aortic stiffness measured by pulse wave velocity in rats. Cardiovascular research 51: 351-358, 2001. 
14. Fleenor BS, Eng JS, Sindler AL, Pham BT, Kloor JD, and Seals DR. Superoxide signaling in perivascular adipose tissue promotes age-related artery stiffness. Aging cell 13: 576$578,2014$.

15. Gao YJ, Lu C, Su LY, Sharma AM, and Lee RM. Modulation of vascular function by perivascular adipose tissue: the role of endothelium and hydrogen peroxide. British journal of pharmacology 151: 323-331, 2007.

16. Gearing AJ, Beckett P, Christodoulou M, Churchill M, Clements J, Davidson AH, Drummond AH, Galloway WA, Gilbert R, Gordon JL, and et al. Processing of tumour necrosis factor-alpha precursor by metalloproteinases. Nature 370: 555-557, 1994.

17. Ghosh AK, Garg SK, Mau T, O'Brien M, Liu J, and Yung R. Elevated Endoplasmic Reticulum Stress Response Contributes to Adipose Tissue Inflammation in Aging. The journals of gerontology Series A, Biological sciences and medical sciences 70: 1320-1329, 2015.

18. Greenstein AS, Khavandi K, Withers SB, Sonoyama K, Clancy O, Jeziorska M, Laing I, Yates AP, Pemberton PW, Malik RA, and Heagerty AM. Local inflammation and hypoxia abolish the protective anticontractile properties of perivascular fat in obese patients. Circulation 119: 1661-1670, 2009.

19. Gurjar MV, Deleon J, Sharma RV, and Bhalla RC. Role of reactive oxygen species in IL-1 beta-stimulated sustained ERK activation and MMP-9 induction. Am J Physiol Heart Circ Physiol 281: H2568-2574, 2001.

20. Gurjar MV, Sharma RV, and Bhalla RC. eNOS gene transfer inhibits smooth muscle cell migration and MMP-2 and MMP-9 activity. Arteriosclerosis, thrombosis, and vascular biology 19: 2871-2877, 1999. 
21. Hohn A, Konig J, and Jung T. Metabolic Syndrome, Redox State, and the Proteasomal System. Antioxidants \& redox signaling 25: 902-917, 2016.

22. Isenberg JS, Martin-Manso G, Maxhimer JB, and Roberts DD. Regulation of nitric oxide signalling by thrombospondin 1: implications for anti-angiogenic therapies. Nature reviews Cancer 9: 182-194, 2009.

\section{Jenkins GM, Crow MT, Bilato C, Gluzband Y, Ryu WS, Li Z, Stetler-Stevenson W,} Nater C, Froehlich JP, Lakatta EG, and Cheng L. Increased expression of membrane-type matrix metalloproteinase and preferential localization of matrix metalloproteinase- 2 to the neointima of balloon-injured rat carotid arteries. Circulation 97: 82-90, 1998.

24. Katakam PV, Snipes JA, Tulbert CD, Mayanagi K, Miller AW, and Busija DW. Impaired endothelin-induced vasoconstriction in coronary arteries of Zucker obese rats is associated with uncoupling of $[\mathrm{Ca} 2+] \mathrm{i}$ signaling. American journal of physiology Regulatory, integrative and comparative physiology 290: R145-153, 2006.

25. Kim YS, Morgan MJ, Choksi S, and Liu ZG. TNF-induced activation of the Nox1 NADPH oxidase and its role in the induction of necrotic cell death. Molecular cell 26: 675-687, 2007.

26. Lakatta EG and Levy D. Arterial and cardiac aging: major shareholders in cardiovascular disease enterprises: Part I: aging arteries: a "set up" for vascular disease. Circulation 107: 139-146, 2003.

\section{Laursen JB, Somers M, Kurz S, McCann L, Warnholtz A, Freeman BA, Tarpey M,} Fukai T, and Harrison DG. Endothelial regulation of vasomotion in apoE-deficient mice: implications for interactions between peroxynitrite and tetrahydrobiopterin. Circulation 103: 1282-1288, 2001. 
28. Levy BI, Ambrosio G, Pries AR, and Struijker-Boudier HA. Microcirculation in hypertension: a new target for treatment? Circulation 104: 735-740, 2001.

29. Li JM, Fan LM, Christie MR, and Shah AM. Acute tumor necrosis factor alpha signaling via NADPH oxidase in microvascular endothelial cells: role of p47phox phosphorylation and binding to TRAF4. Molecular and cellular biology 25: 2320-2330, 2005.

30. Lin Y, Berg AH, Iyengar P, Lam TK, Giacca A, Combs TP, Rajala MW, Du X, Rollman B, Li W, Hawkins M, Barzilai N, Rhodes CJ, Fantus IG, Brownlee M, and Scherer PE. The hyperglycemia-induced inflammatory response in adipocytes: the role of reactive oxygen species. $J$ Biol Chem 280: 4617-4626, 2005.

31. Mandler WK, Nurkiewicz TR, Porter DW, and Olfert IM. Thrombospondin-1 mediates multi-walled carbon nanotube induced impairment of arteriolar dilation. Nanotoxicology 11: 112-122, 2017.

32. Mikolajczyk TP, Nosalski R, Szczepaniak P, Budzyn K, Osmenda G, Skiba D, Sagan A, Wu J, Vinh A, Marvar PJ, Guzik B, Podolec J, Drummond G, Lob HE, Harrison DG, and Guzik TJ. Role of chemokine RANTES in the regulation of perivascular inflammation, T-cell accumulation, and vascular dysfunction in hypertension. FASEB journal : official publication of the Federation of American Societies for Experimental Biology 30: 19871999, 2016.

33. Mingorance C, Alvarez de Sotomayor M, Jimenez-Palacios FJ, Callejon Mochon M, Casto C, Marhuenda E, and Herrera MD. Effects of chronic treatment with the CB1 antagonist, rimonabant on the blood pressure, and vascular reactivity of obese Zucker rats. Obesity 17: 1340-1347, 2009. 
34. O'Rourke M. Arterial stiffness, systolic blood pressure, and logical treatment of arterial hypertension. Hypertension 15: 339-347, 1990.

35. Otoda T, Takamura T, Misu H, Ota T, Murata S, Hayashi H, Takayama H, Kikuchi A, Kanamori T, Shima KR, Lan F, Takeda T, Kurita S, Ishikura K, Kita Y, Iwayama K, Kato K, Uno M, Takeshita Y, Yamamoto M, Tokuyama K, Iseki S, Tanaka K, and Kaneko S. Proteasome dysfunction mediates obesity-induced endoplasmic reticulum stress and insulin resistance in the liver. Diabetes 62: 811-824, 2013.

36. Parameswaran N and Patial S. Tumor necrosis factor-alpha signaling in macrophages. Critical reviews in eukaryotic gene expression 20: 87-103, 2010.

37. Raychaudhuri B, Fisher CJ, Farver CF, Malur A, Drazba J, Kavuru MS, and Thomassen MJ. Interleukin 10 (IL-10)-mediated inhibition of inflammatory cytokine production by human alveolar macrophages. Cytokine 12: 1348-1355, 2000.

38. Roderfeld M, Graf J, Giese B, Salguero-Palacios R, Tschuschner A, Muller-Newen G, and Roeb E. Latent MMP-9 is bound to TIMP-1 before secretion. Biological chemistry 388: 1227-1234, 2007.

39. Saji N, Kimura K, Shimizu H, and Kita Y. Association between silent brain infarct and arterial stiffness indicated by brachial-ankle pulse wave velocity. Intern Med 51: 1003-1008, 2012.

40. Sakamoto T, Takahashi N, Sawaragi Y, Naknukool S, Yu R, Goto T, and Kawada T. Inflammation induced by RAW macrophages suppresses UCP1 mRNA induction via ERK activation in 10T1/2 adipocytes. Am J Physiol Cell Physiol 304: C729-738, 2013.

41. Saperstein S, Chen L, Oakes D, Pryhuber G, and Finkelstein J. IL-1beta augments TNF-alpha-mediated inflammatory responses from lung epithelial cells. Journal of interferon \& 
cytokine research : the official journal of the International Society for Interferon and Cytokine Research 29: 273-284, 2009.

42. Schonbeck U, Mach F, and Libby P. Generation of biologically active IL-1 beta by matrix metalloproteinases: a novel caspase-1-independent pathway of IL-1 beta processing. Journal of immunology 161: 3340-3346, 1998.

43. Sen CK and Packer L. Antioxidant and redox regulation of gene transcription. FASEB journal : official publication of the Federation of American Societies for Experimental Biology 10: 709-720, 1996.

44. Serpillon S, Floyd BC, Gupte RS, George S, Kozicky M, Neito V, Recchia F, Stanley W, Wolin MS, and Gupte SA. Superoxide production by NAD(P)H oxidase and mitochondria is increased in genetically obese and hyperglycemic rat heart and aorta before the development of cardiac dysfunction. The role of glucose-6-phosphate dehydrogenase-derived NADPH. American journal of physiology Heart and circulatory physiology 297: H153-162, 2009.

45. Shimizu I, Aprahamian T, Kikuchi R, Shimizu A, Papanicolaou KN, MacLauchlan S, Maruyama S, and Walsh K. Vascular rarefaction mediates whitening of brown fat in obesity. The Journal of clinical investigation 124: 2099-2112, 2014.

46. Smith DM, Fraga H, Reis C, Kafri G, and Goldberg AL. ATP binds to proteasomal ATPases in pairs with distinct functional effects, implying an ordered reaction cycle. Cell 144: 526-538, 2011.

47. Turner NA, Mughal RS, Warburton P, O'Regan DJ, Ball SG, and Porter KE. Mechanism of TNFalpha-induced IL-1alpha, IL-1beta and IL-6 expression in human cardiac fibroblasts: effects of statins and thiazolidinediones. Cardiovascular research 76: 81-90, 2007. 
48. Vasquez-Vivar J, Kalyanaraman B, Martasek P, Hogg N, Masters BS, Karoui H, Tordo P, and Pritchard KA, Jr. Superoxide generation by endothelial nitric oxide synthase: the influence of cofactors. Proceedings of the National Academy of Sciences of the United States of America 95: 9220-9225, 1998.

49. Virdis A, Duranti E, Rossi C, Dell'Agnello U, Santini E, Anselmino M, Chiarugi M, Taddei S, and Solini A. Tumour necrosis factor-alpha participates on the endothelin-1/nitric oxide imbalance in small arteries from obese patients: role of perivascular adipose tissue. European heart journal 36: 784-794, 2015.

50. Wang Y, Wang X, Lau WB, Yuan Y, Booth D, Li JJ, Scalia R, Preston K, Gao E, Koch W, and Ma XL. Adiponectin inhibits tumor necrosis factor-alpha-induced vascular inflammatory response via caveolin-mediated ceramidase recruitment and activation. Circulation research 114: 792-805, 2014.

51. Wang ZV and Scherer PE. Adiponectin, cardiovascular function, and hypertension. Hypertension 51: 8-14, 2008.

52. Wilkinson IB, Franklin SS, and Cockcroft JR. Nitric oxide and the regulation of large artery stiffness: from physiology to pharmacology. Hypertension 44: 112-116, 2004.

53. Wu HT, Sie SS, Kuan TC, and Lin CS. Identifying the regulative role of NF-kappaB binding sites within promoter region of human matrix metalloproteinase 9 (mmp-9) by TNFalpha induction. Applied biochemistry and biotechnology 169: 438-449, 2013.

54. Yang B and Rizzo V. TNF-alpha potentiates protein-tyrosine nitration through activation of NADPH oxidase and eNOS localized in membrane rafts and caveolae of bovine aortic endothelial cells. Am J Physiol Heart Circ Physiol 292: H954-962, 2007. 


\section{Yasmin, McEniery CM, Wallace S, Dakham Z, Pulsalkar P, Maki-Petaja K, Ashby}

MJ, Cockcroft JR, and Wilkinson IB. Matrix metalloproteinase-9 (MMP-9), MMP-2, and serum elastase activity are associated with systolic hypertension and arterial stiffness. Arteriosclerosis, thrombosis, and vascular biology 25: 372, 2005.

56. Zemse SM, Chiao CW, Hilgers RH, and Webb RC. Interleukin-10 inhibits the in vivo and in vitro adverse effects of TNF-alpha on the endothelium of murine aorta. Am J Physiol Heart Circ Physiol 299: H1160-1167, 2010.

57. Zhang M, Kho AL, Anilkumar N, Chibber R, Pagano PJ, Shah AM, and Cave AC. Glycated proteins stimulate reactive oxygen species production in cardiac myocytes: involvement of Nox2 (gp91phox)-containing NADPH oxidase. Circulation 113: 1235-1243, 2006.

\section{Figure Legends}

Figure 3.1. Relative gene expression of tPVAT. The graph shows relative gene expression in OZR tPVAT compared to LZR tPVAT for A) phenotype and immune markers B) oxidative and inflammatory genes, and C) and anti-inflammatory and oxidative defense markers. Data expressed as Mean \pm SEM, *denotes significant difference in OZR vs. LZR, minimum of 2-fold change and t-test $\mathrm{p}<0.05, \mathrm{n}=3$. UCP-1, uncoupling protein- 1 ; $\mathrm{CD}$, cluster of differentiation; NOX2, NADPH oxidase 2 catalytic subunit (GP91); p47phox, NADPH oxidase 2 intracellular regulatory subunit; TNF, tumor necrosis factor; CCL5, Chemokine (C-C motif) ligand 5; IL, interleukin; TSP-1, thrombospondin 1; IFN- $\gamma$, interferon gamma; CCR, C-C motif chemokine receptor; AdipoQ, adiponectin; NFR2, nuclear factor (erythroid 2)-like 2; Keap1, kelch-like ECH associated protein 1, SOD, superoxide dismutase; GSR, glutathione reductase; CAT, catalase. 
Figure 3.2. tPVAT ROS formation SOD defense and proteasome function. A) DHE measured ROS production $(n=8)$ and B) relative SOD activity $(n=5)$ in tPVAT. C) Proteasome function measured across all 3 active sites $(n=8)$; LLVY (chymotrypsin-like), RLR (trypsin-like) and nLPnLD (peptidylglutamyl-peptide hydrolyzing) and D) levels of ubiquitin and the 20S proteasome from tPVAT homogenates $(n=5)$. Data expressed as Mean \pm SEM, *denotes significant difference in OZR vs. LZR measured by t-test, $\mathrm{p}<0.05$.

Figure 3.3. Cytokine profile of tPVAT. A \& B) tPVAT immuno-attractive and inflammatory cytokines and C \& D) levels of anti-inflammatory cytokines ( $\mathrm{n}=5)$, HMW: high molecular weight. Data expressed as Mean \pm SEM *denotes a statistically significant change in OZR vs. LZR determined by t-test, $\mathrm{p}<0.05 . \mathrm{KC} / \mathrm{GRO}$, chemokine (C-C motif) ligand 1; MCP-1, monocyte chemoattractant protein-1; TNF $\alpha$, tumor necrosis factor alpha; IL, interleukin; IFN- $\gamma$, interferon gamma; TSP-1, thrombospondin 1; HMW adiponectin, high molecular weight adiponectin

Figure 3.4. OZR-tPVAT role in activating aortic ROS production and reducing nitric oxide. A) tPVAT effect on aortic (Ao) ROS production measured by DHE assay $(n=8)$, and B) relative SOD activity of aortic homogenates $(n=4) . C \&$ D) Effect of tPVAT on aortic NO production and EDD and $(n=16) . E, F, \& G)$ the effect of crossover treating LZR rings with OZR-tPVAT and OZR rings with LZR-tPVAT had on aortic ROS, NO, and EDD ( $\mathrm{n}=8)$. Data expressed as Mean \pm SEM *denotes significance between LZR and OZR, \# denotes a significant difference between $\operatorname{tPVAT}$ and respective control, and ${ }^{\wedge}$ denotes a significant difference of drug treatment 
compared to respective tPVAT. SOD activity was analyzed by t-test while drug treatments and aortic reactivity were analyzed by repeated measures ANOVA. Pe, Phenylephrine; MCh, Methacholine.

Figure 3.5. Effect of TNF $\alpha-n A B$ on tPVAT and tPVAT mediated aortic function. A) Effect of $\mathrm{TNF} \alpha \mathrm{AB}$ treatment on activation of NF- $\mathrm{KB}(\mathrm{n}=6)$ and the $\mathrm{B})$ change in tPVAT gene expression following $\mathrm{TNF} \alpha-\mathrm{nAB}$ treatment $(\mathrm{n}=3)$. C) ROS production in both tPVAT and aorta following tPVAT TNF $\alpha$-nAB treatment $(n=8)$. D) The effect of TNF $\alpha-n A B$ treated OZR-tPVAT on both LZR and OZR aortic rings NO production $(n=5-8)$, and E \& F) EDD ( $\mathrm{n}=5-8)$. Data expressed as $\mathrm{Mean} \pm \mathrm{SEM} \wedge$ denotes significant effect of $\mathrm{TNF} \alpha-\mathrm{nAB}$ compared to control assessed by repeated measures ANOVA, $\mathrm{p}<0.05$. Pe, Phenylephrine; MCh, Methacholine; TNF, tumor necrosis factor; NOX2, NADPH oxidase 2 catalytic subunit (GP91); p47phox, NADPH oxidase 2 intracellular regulatory subunit; TSP-1, thrombospondin 1; IL, interleukin; IFN- $\gamma$, interferon gamma; NFR2, nuclear factor (erythroid 2)-like 2; AdipoQ, adiponectin MMP, matrix metalloproteinase; TIMP, tissue inhibitor of metalloproteinase.

Figure 3.6. Role of tPVAT in aortic stiffness. A) Aortic elastic modulus in LZR and OZR rings without culture experiments ( $\mathrm{n}=8)$, with B) tPVAT gene expression of remodeling factors $(\mathrm{n}=3)$ and C \& D) tPVAT tissue levels of TIMP-1 and relative activity of MMP9 form LZR, OZR, and $\mathrm{OZR}+\mathrm{TNF} \alpha-\mathrm{nAB}$ tPVAT exudate $(\mathrm{n}=5)$. E) Elastic modulus of LZR aortic rings following 72 hours culture with media, LZR-tPVAT, OZR-tPVAT, or OZR-tPVAT+TNF $\alpha$-nAB (n= 3-4). Data expressed as Mean \pm SEM *denotes significant change between OZR and LZR and ^denotes significant effect of TNF $\alpha-\mathrm{nAB}$ treatment compared to OZR. A, B, \& D analyzed by t-test and 
$\mathrm{C} \& \mathrm{E}$ analyzed by 2-way repeated measures ANOVA, $\mathrm{p}<0.05 . \mathrm{MMP}$, matrix metalloproteinase; TIMP, tissue inhibitor of metalloproteinase 
Figures

Figure 3.1. Relative gene expression of tPVAT

A

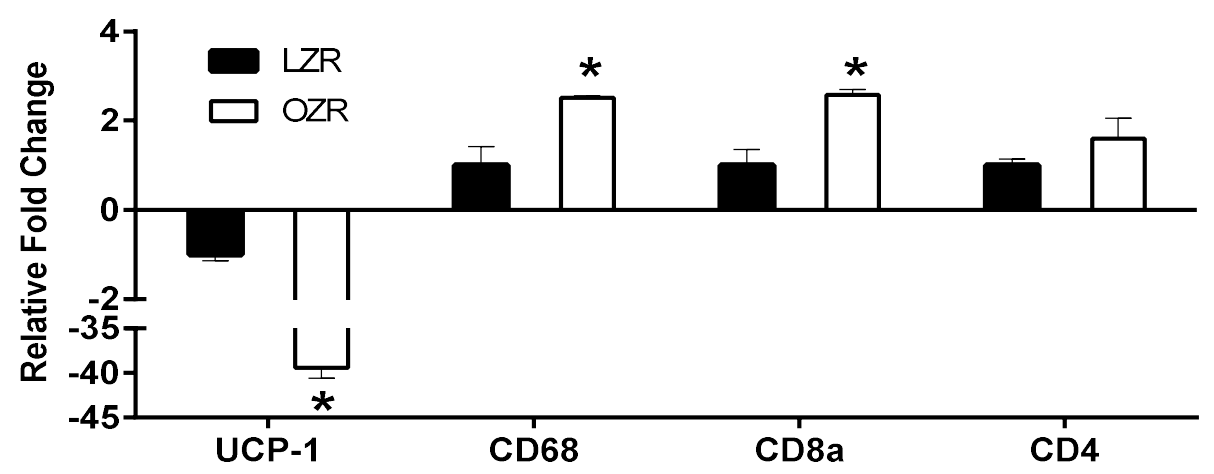

B

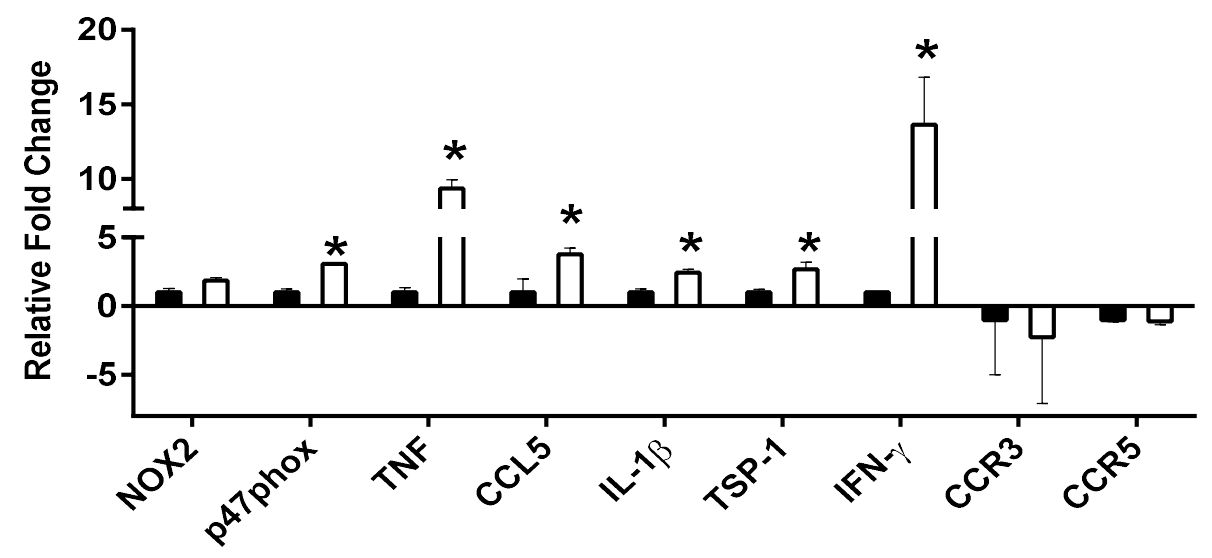

C

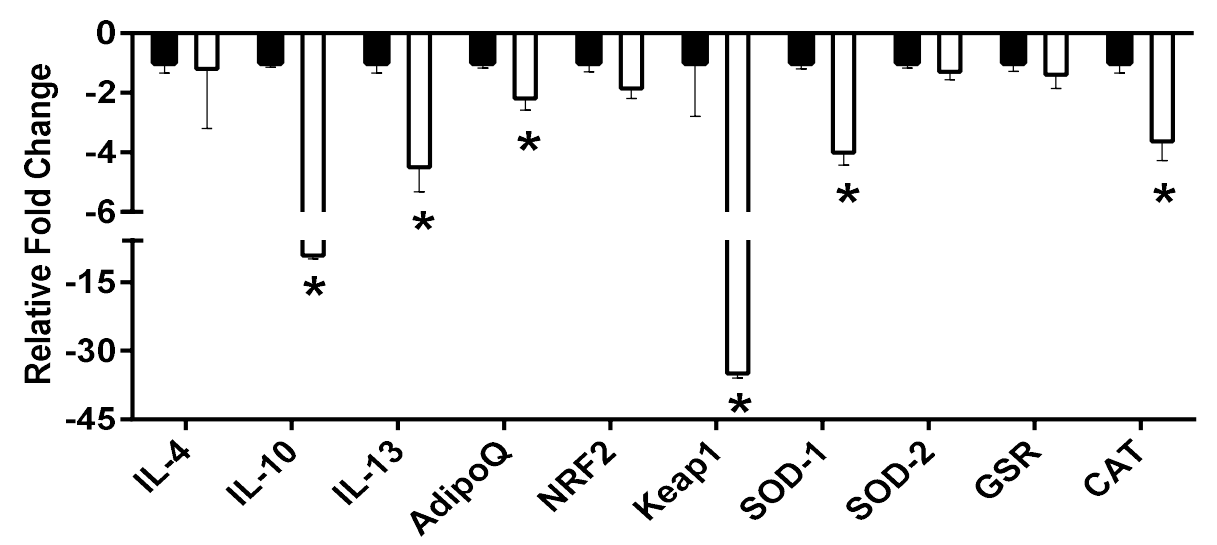


A

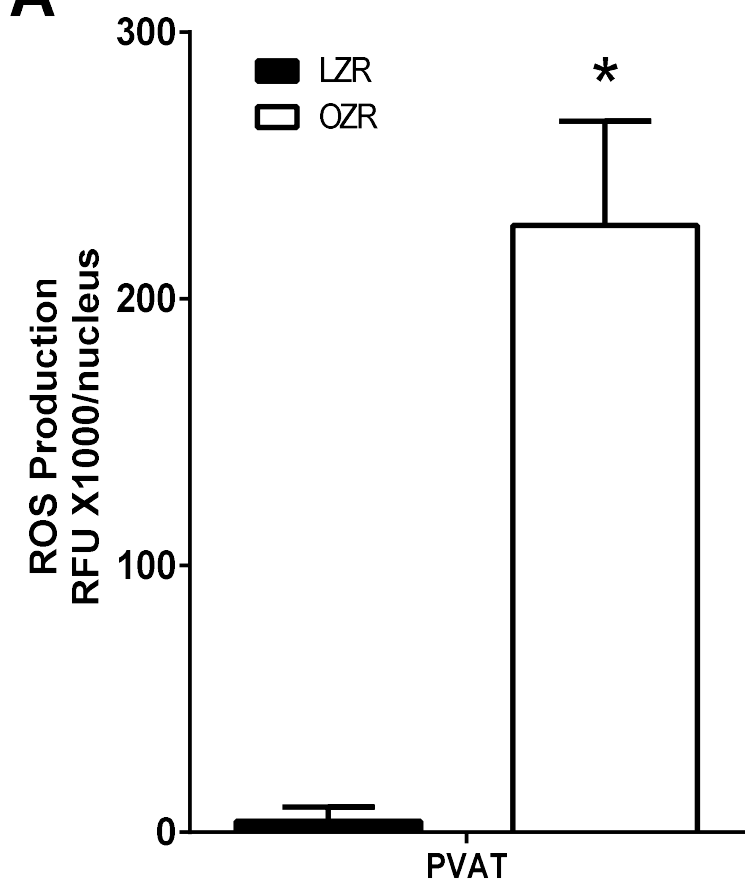

B

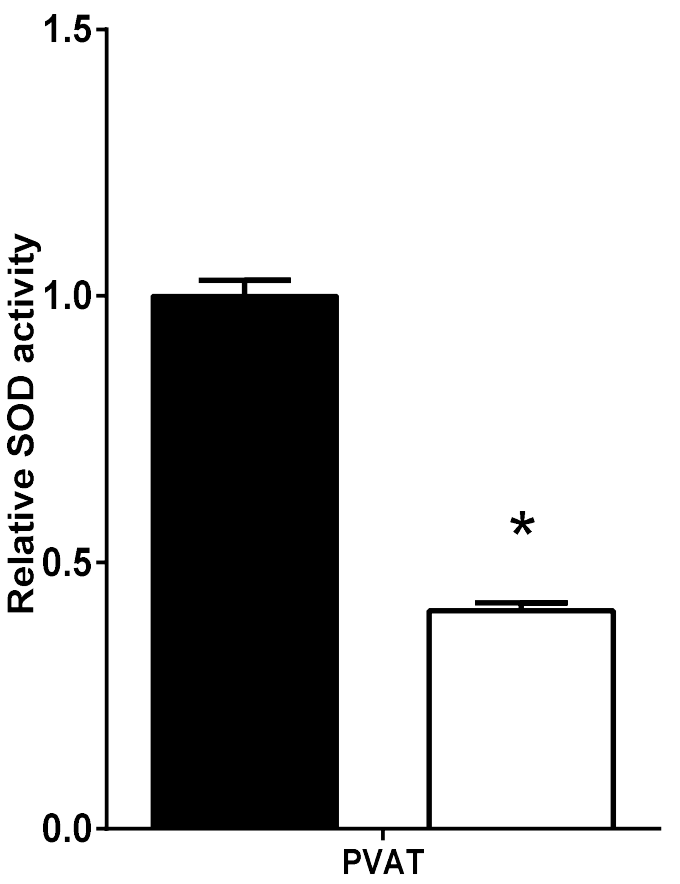

D

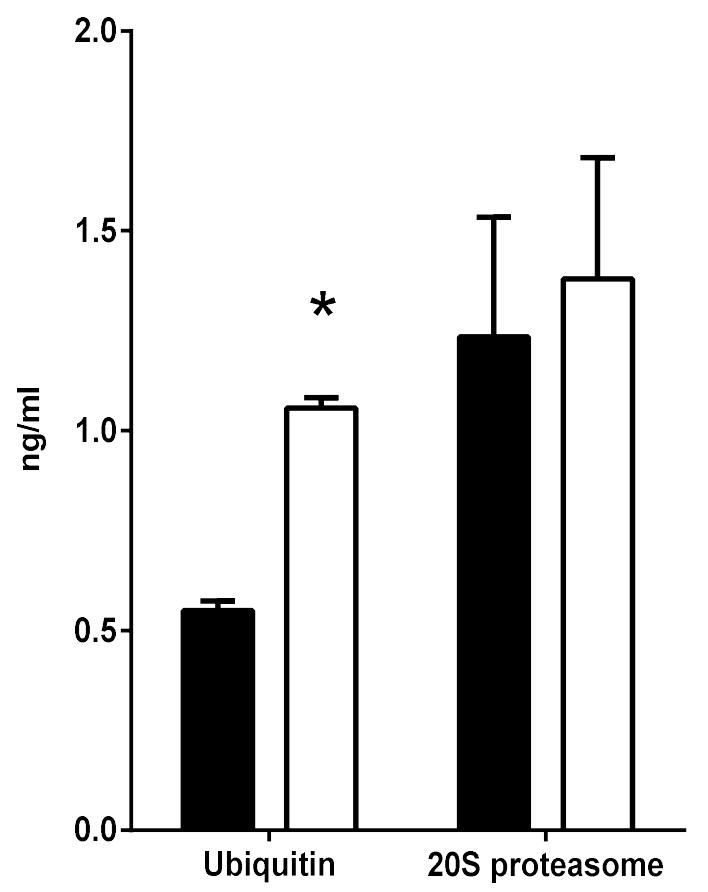


A
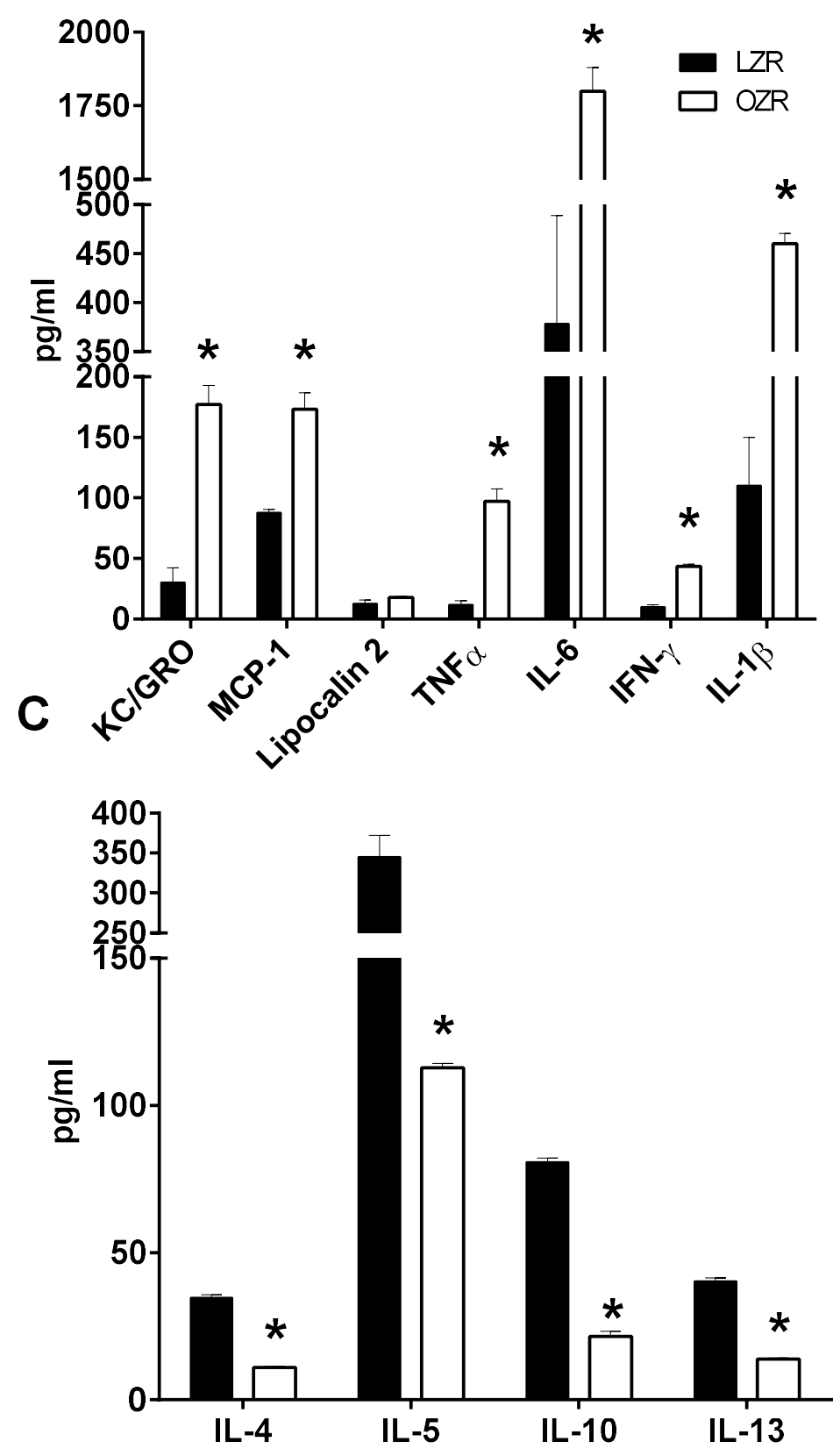

B
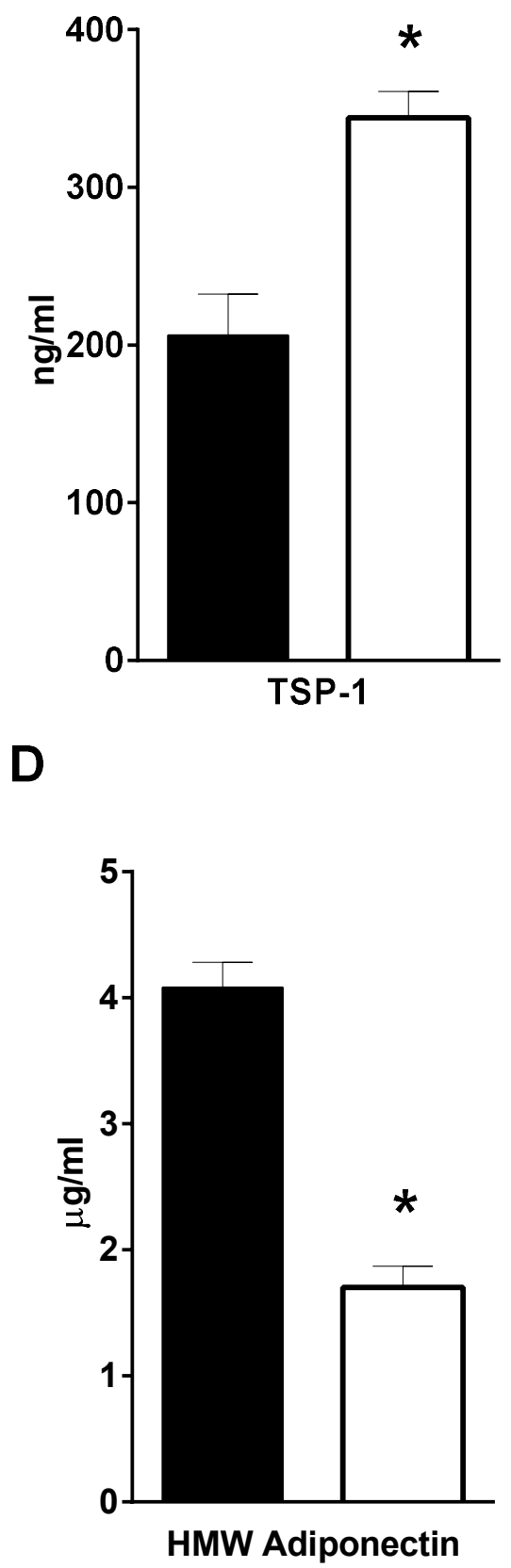
Figure 3.4. OZR-tPVAT role in activating aortic ROS production and reducing nitric oxide
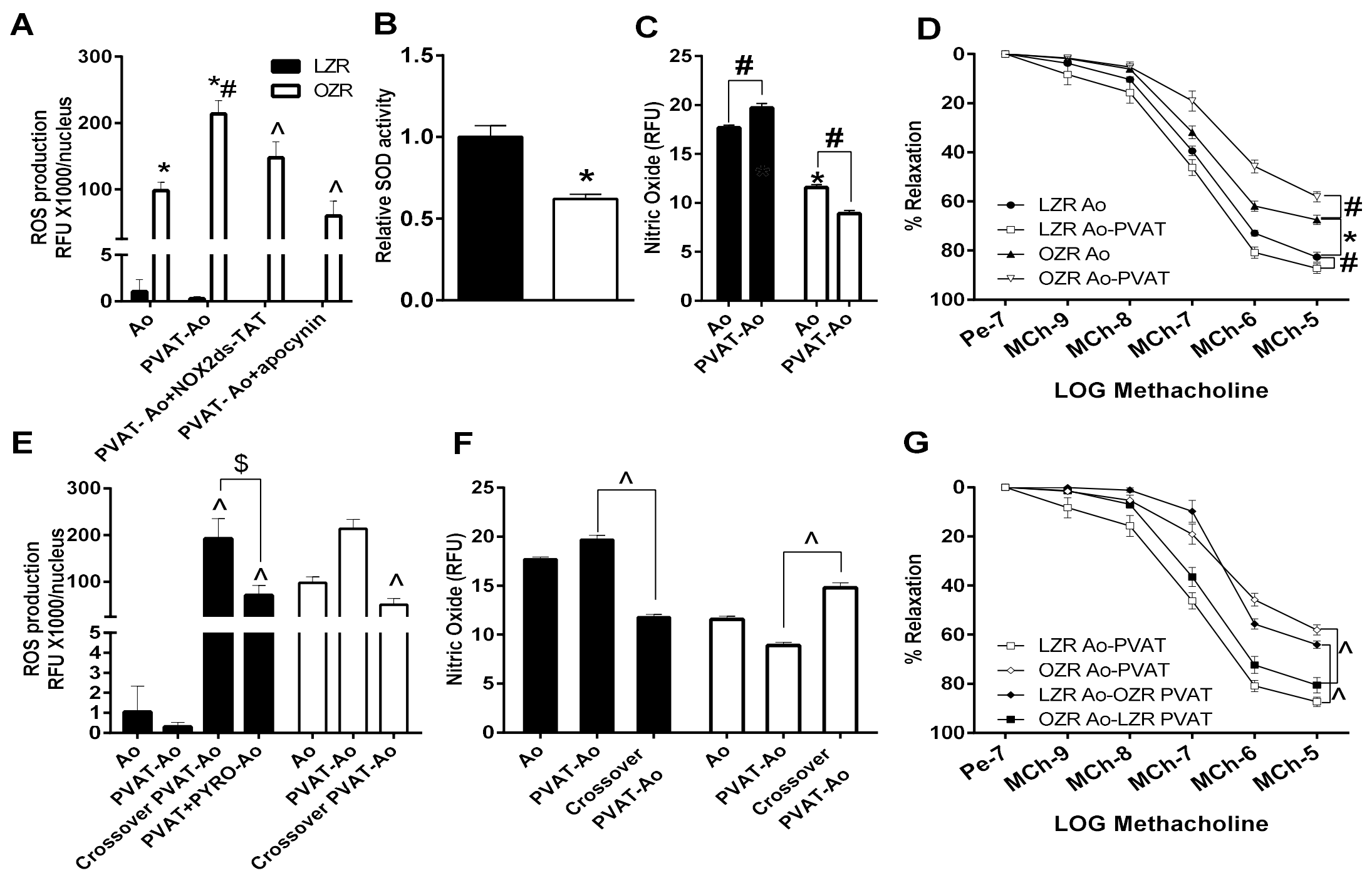

F

G
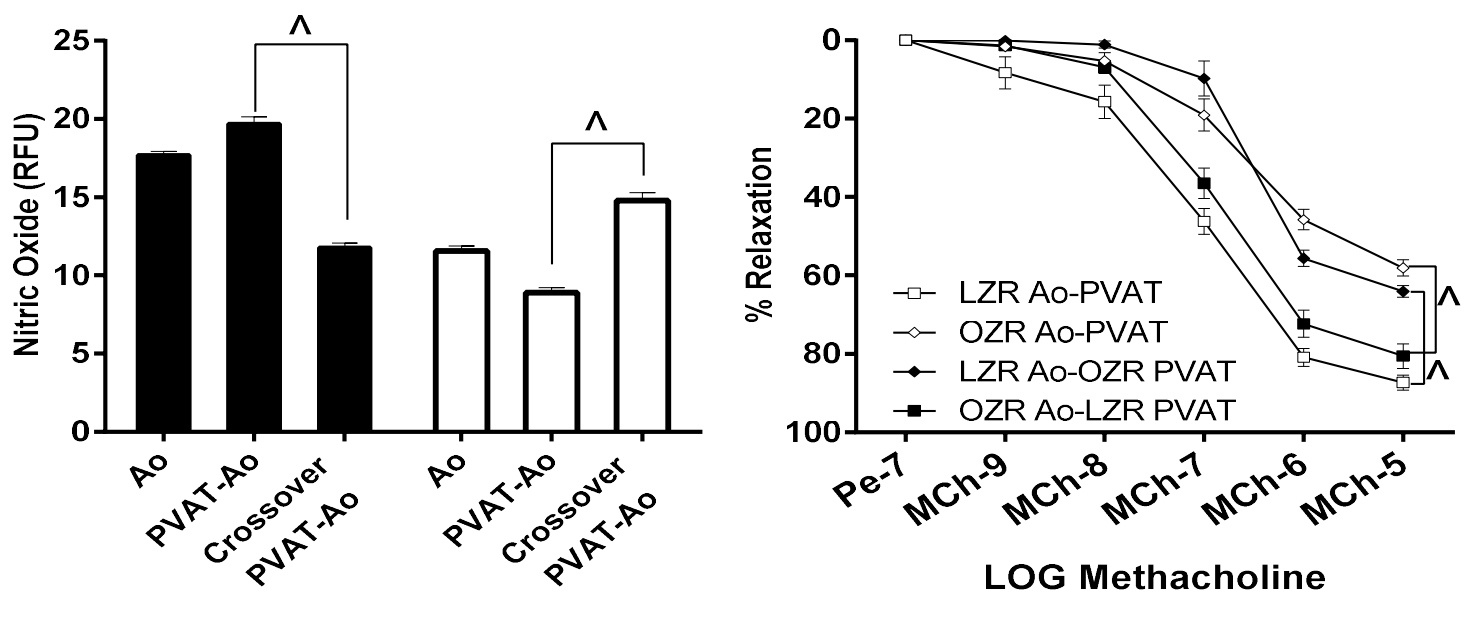
Figure 3.5. Effect of TNF $\alpha-n A B$ on $t P V A T$ and $t P V A T$ mediated aortic function
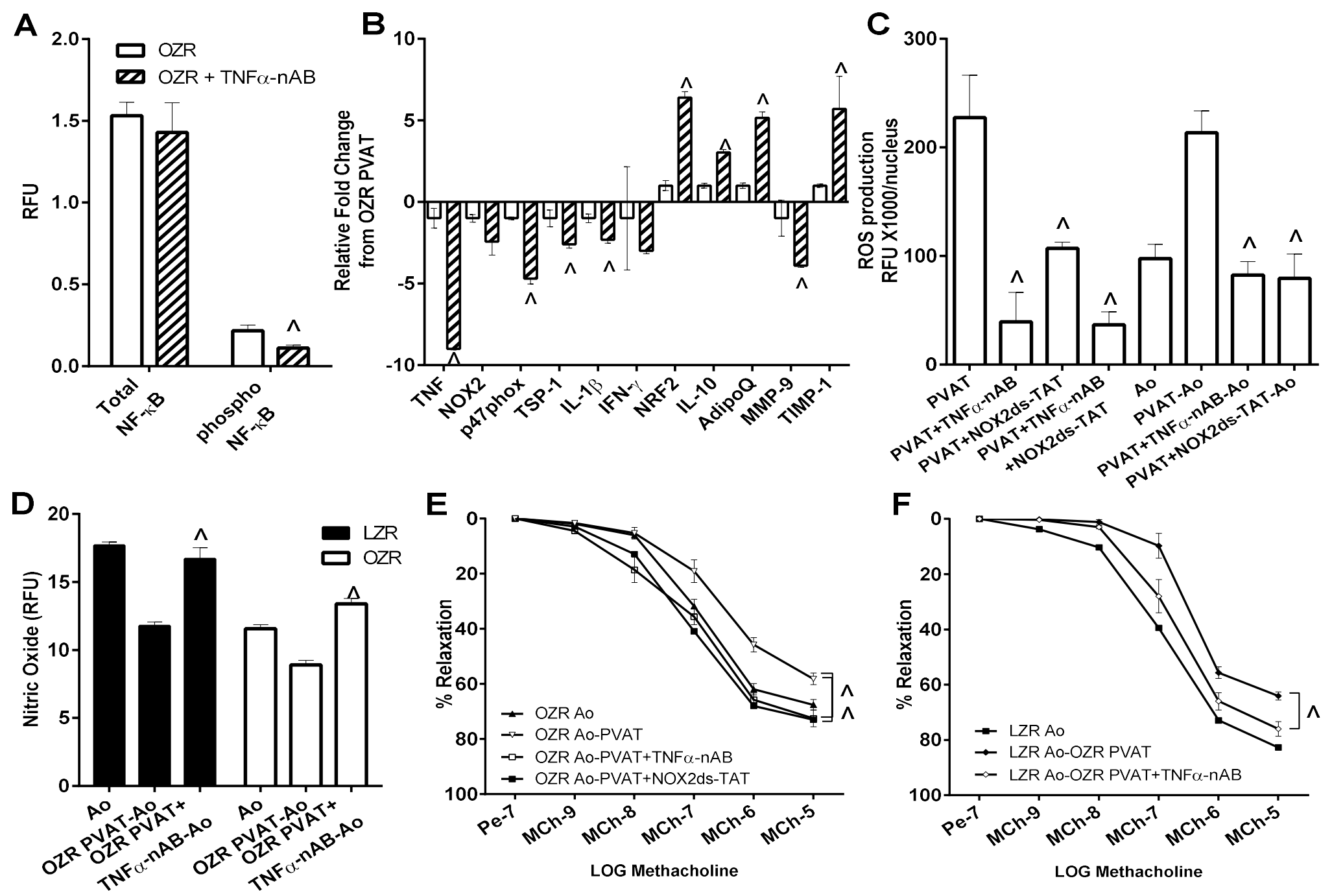

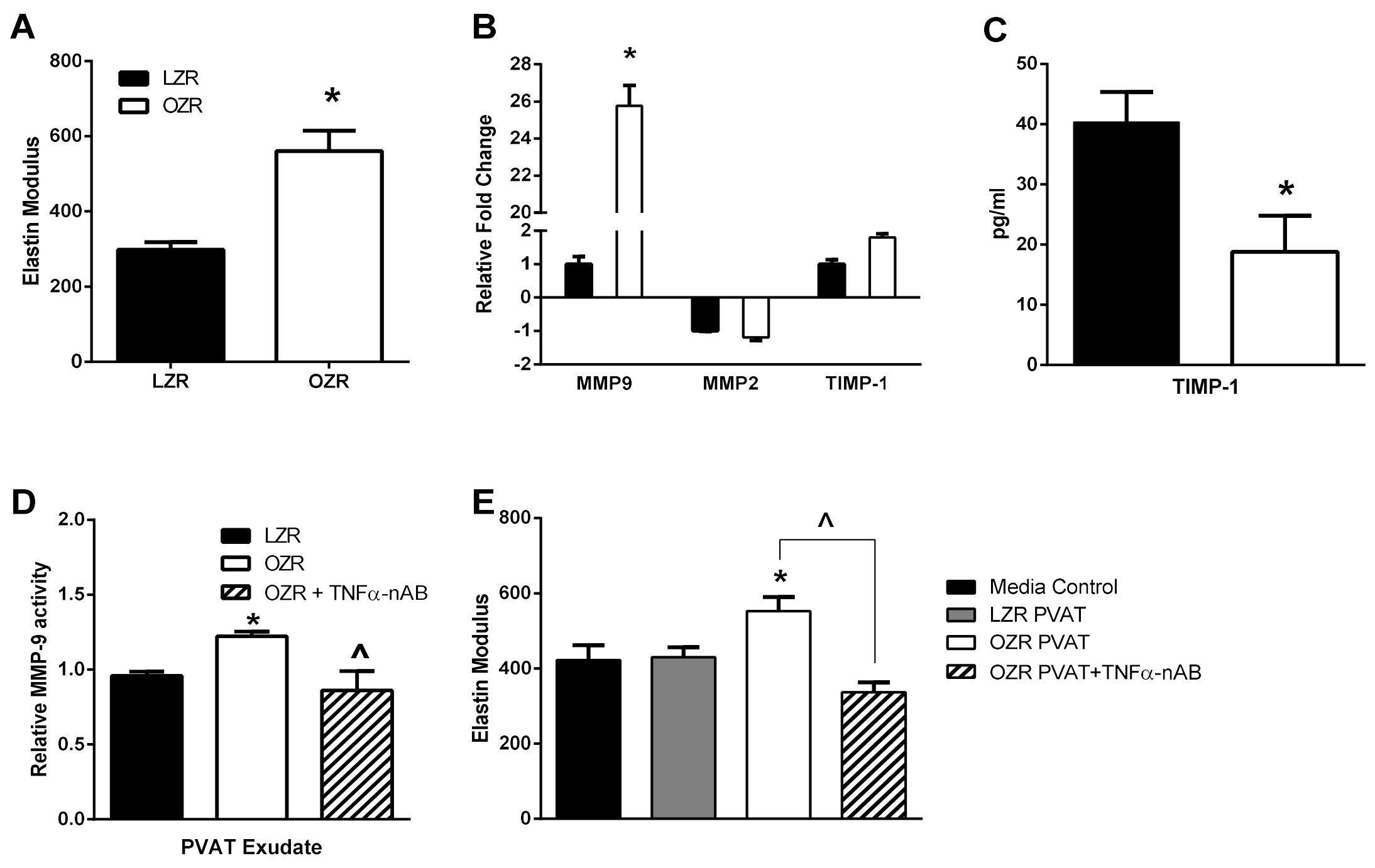


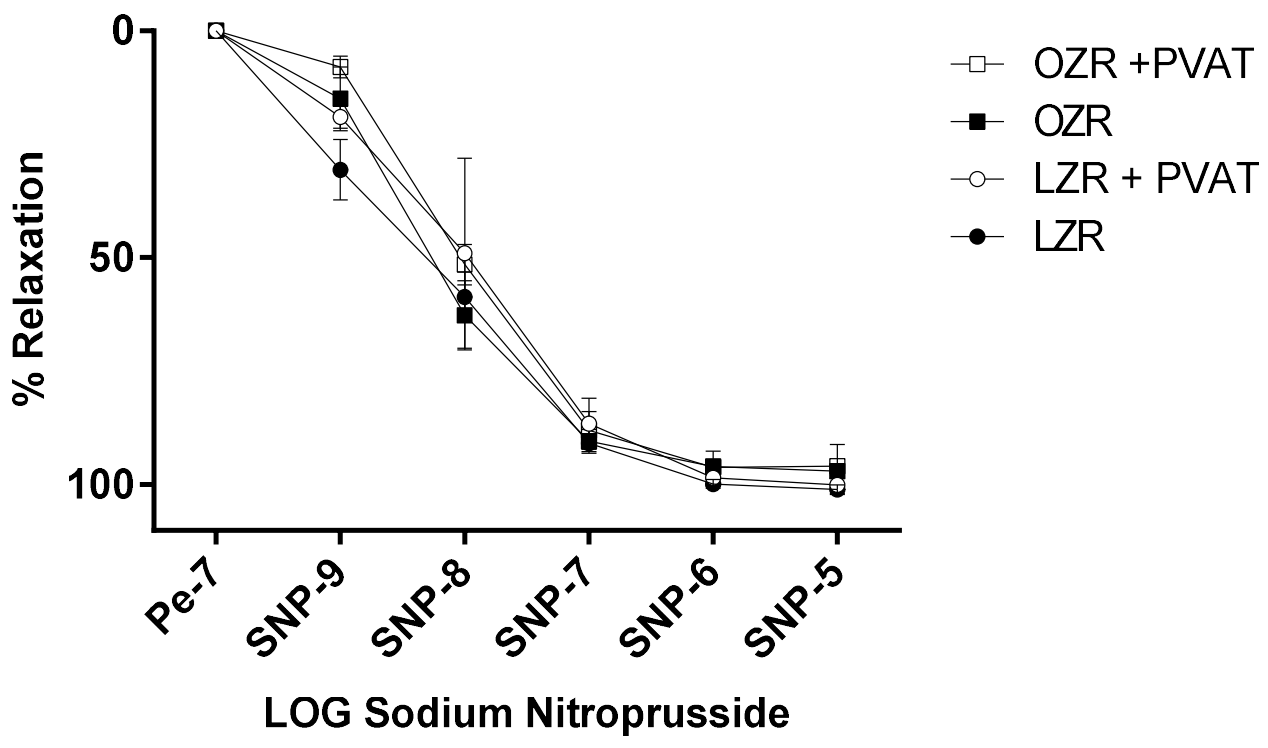

Figure 1S. endothelial independent dilation to sodium nitroprusside 


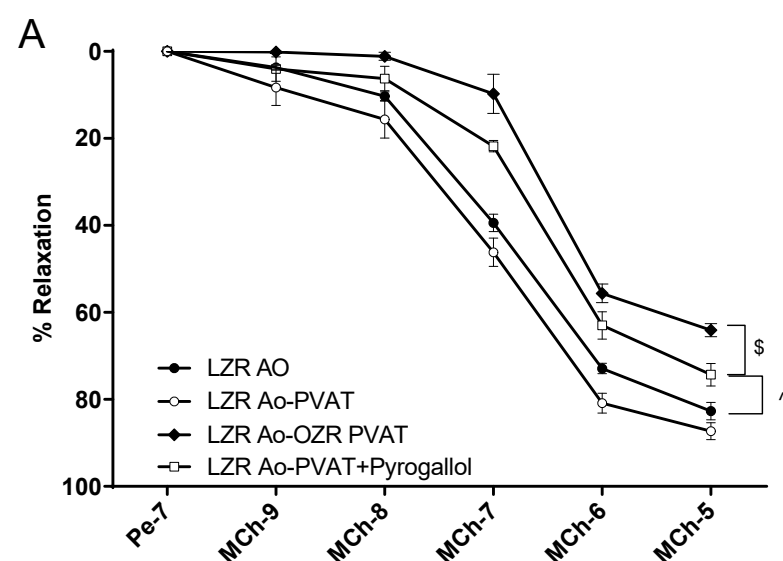

C

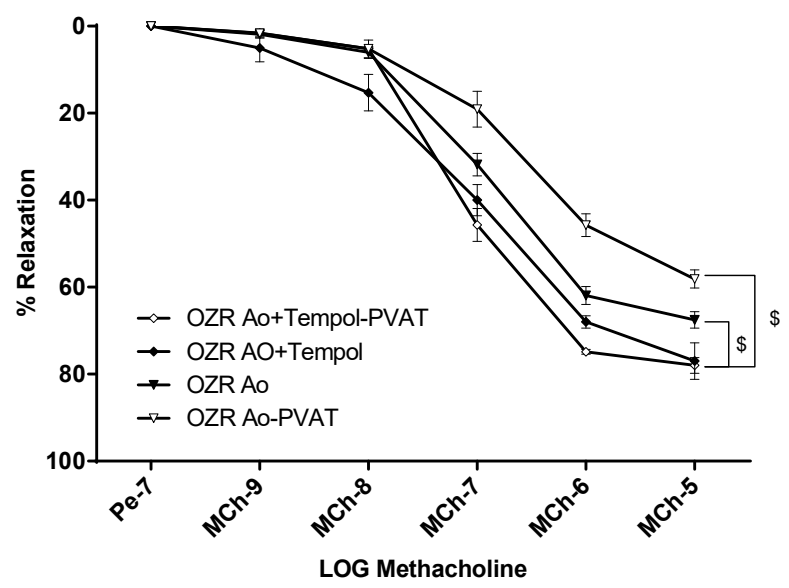

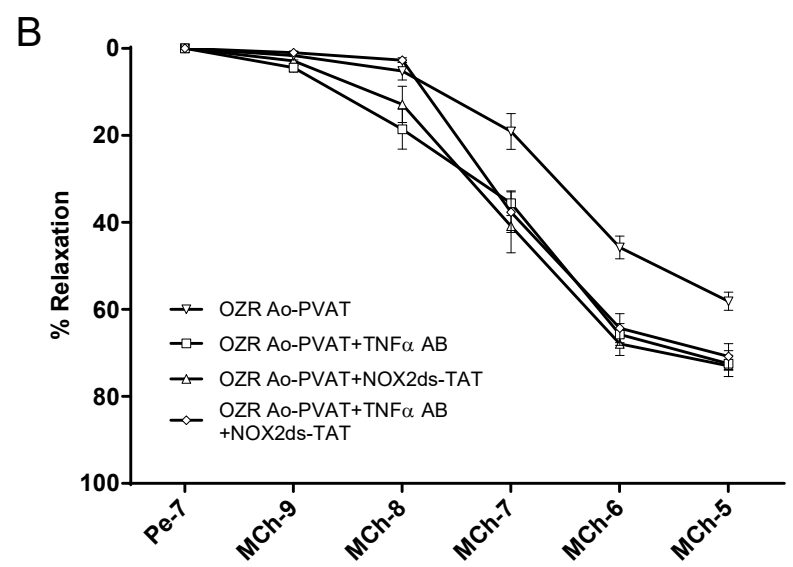

LOG Methacholine

Figure 2S. tPVAT treatments and its effects on EDD. A) Effect of creating an oxidative environement in LZR TPVAT and the effect on EDD ( $n=4)$. B) The effect of co-treatment with TNF $\alpha$ $A B$ and NOX2ds-TAT. C) Effect of treating OZR aortic rings with the anti-oxidant Tempol with and without subsequent incubation with tPVAT media. \$ denotes significant effect of treatment and ${ }^{\wedge}$ denote significance form OZR tPVAT crossover. 
Supplemental Image 1. Representative DHE Images

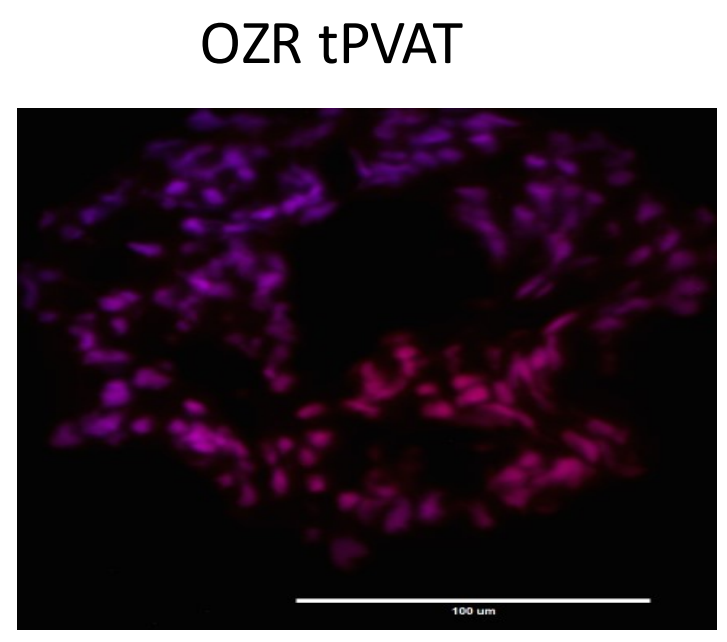

\section{LZR tPVAT}

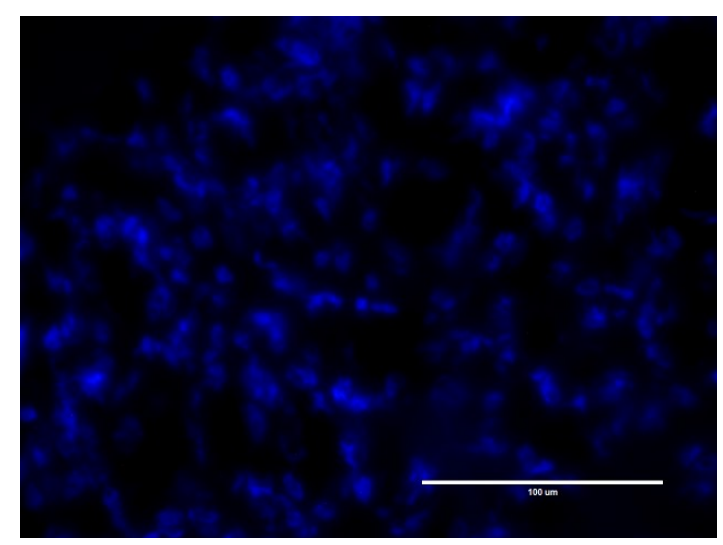


Supplemental image 2. Representative DHE Images

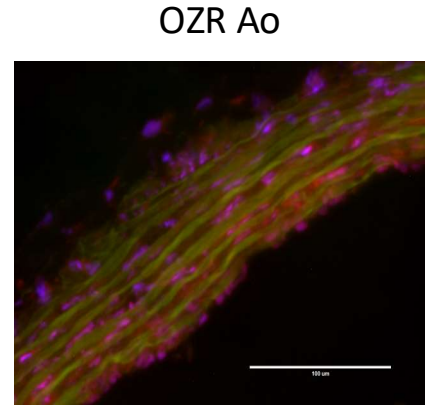

OZR tPVAT-Ao

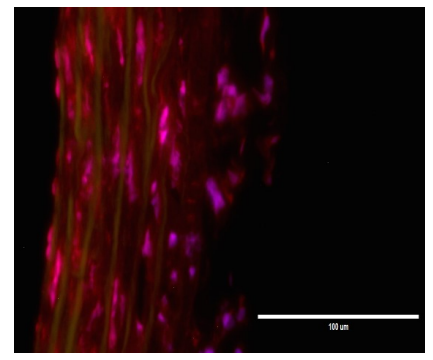

LZR Ao

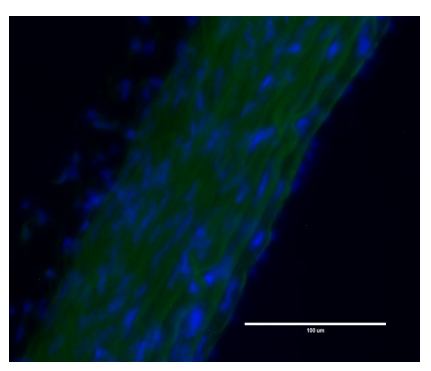

LZR tPVAT-Ao

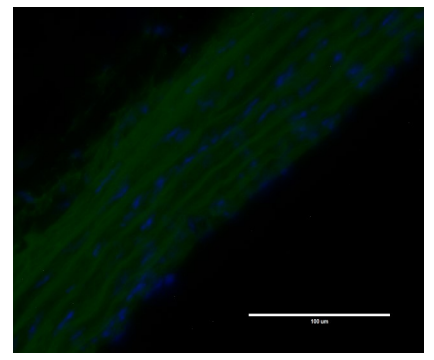

OZR tPVAT-Ao+NOX2ds-TAT

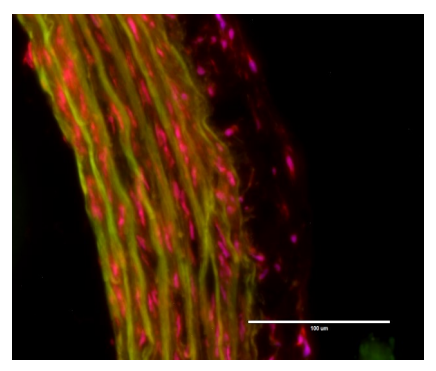

OZR tPVAT-Ao+apocynin

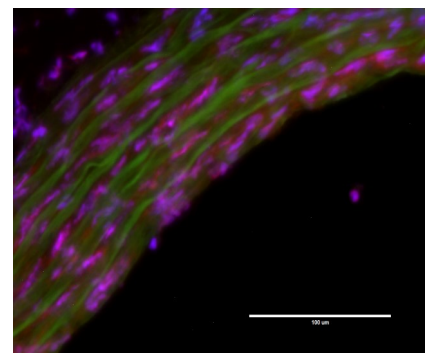


Supplemental Image 3. Representative DHE Images

\section{LZR tPVAT-OZR Ao}

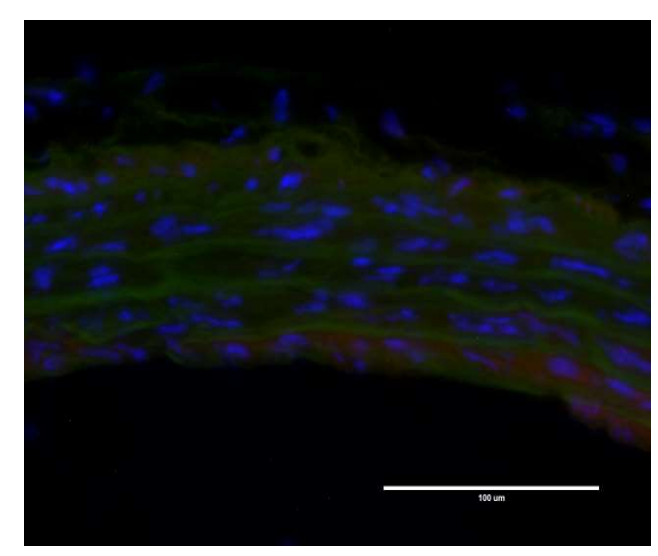

\section{LZR tPVAT+pyrogallol-Ao}

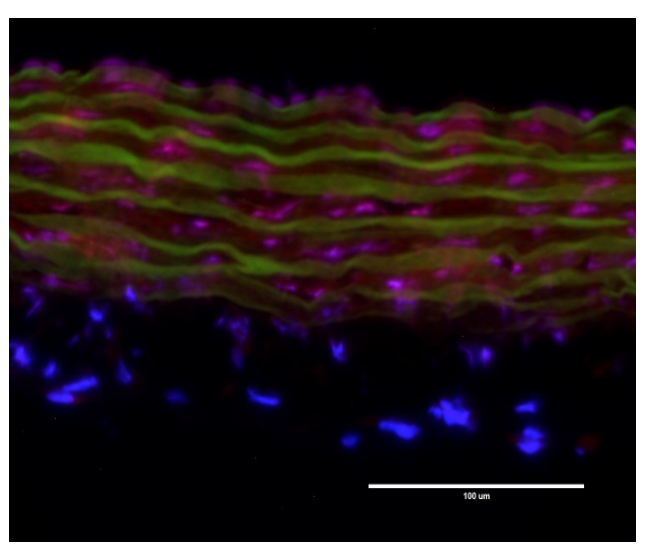

OZR tPVAT-LZR Ao

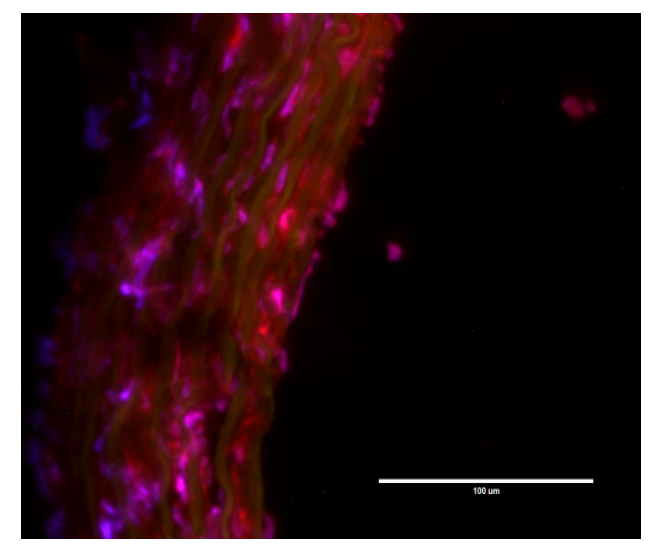


Supplemental Image 4. Representative DHE Images

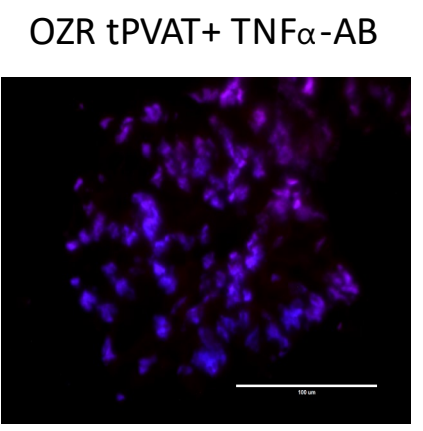

OZR tPVAT+ NOX2ds-TAT \& TNF $\alpha-A B$

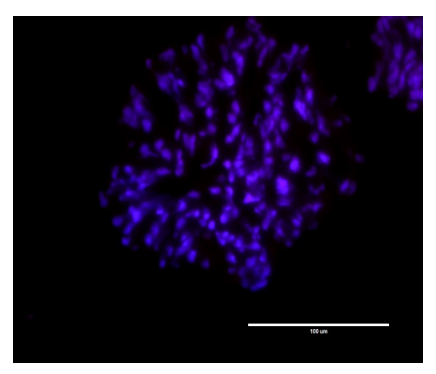

OZR tPVAT+TNF $\alpha-A B-A o$

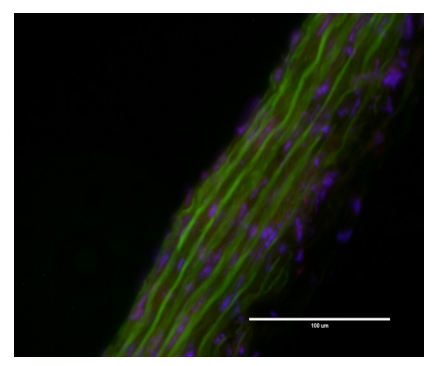

OZR tPVAT+NOX2ds-TAT-Ao

OZR tPVAT+ NOX2ds-TAT

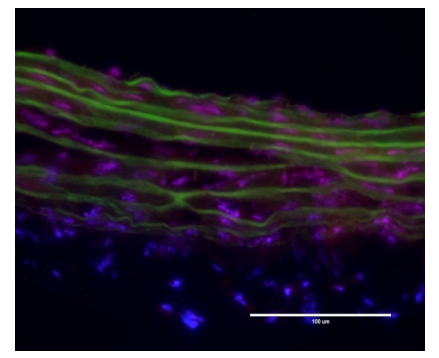




\title{
Chapter 4: Aerobic Exercise Prevents the Impairment of Aortic Function in Metabolic Syndrome: Role of Perivascular Adipose Tissue.
}

\author{
Evan DeVallance ${ }^{1}$, Kayla W Branyan ${ }^{1}$, Kent Lemaster ${ }^{2}$, I. Mark Olfert ${ }^{1}$, David M. Smith ${ }^{3}$, \\ Emidio E Pistilli ${ }^{1}$, Randall Bryner ${ }^{1}$, Jefferson C Frisbee ${ }^{2,4}$, Paul D. Chantler ${ }^{1}$
}

${ }^{1}$ Department of Exercise Physiology, West Virginia University School of Medicine, Morgantown, WV; ${ }^{2}$ Department of Physiology and Pharmacology, Schulich School of Medicine and Dentistry, University of Western Ontario, London, ON, Canada; ${ }^{3}$ Department of Biochemistry, West Virginia University School of Medicine, Morgantown, WV; ${ }^{4}$ Department of Medical Biophysics, Schulich School of Medicine and Dentistry, University of Western Ontario, London, ON, Canada

Running title: Exercise prevents MetS impairment of tPVAT

Corresponding author

Paul D Chantler,

One Medical Center Drive, Morgantown, WV, 26505, pchantler@hsc.wvu.edu,

tel: (304) 293-0646

fax: (304) 293-7105

Keywords: Perivascular adipose tissue, metabolic syndrome, inflammation, NOX2, proteasome, exercise, tumor necrosis factor alpha, aortic stiffness.

Word count: 6871

Total number of figures and tables: 6 Figures, 1 Table 
Abstract: We reported thoracic perivascular adipose tissue (tPVAT) mediates aortic impairment in metabolic syndrome (MetS). With the known benefits of exercise, we aimed to determine the effect of 8-weeks of aerobic exercise training (Ex) on preventing the MetS impairments in tPVAT and tPVAT induced aortic dysfunction. Methods \& Results: Experiments were conducted in lean (LZR) and obese (OZR) Zucker rats (n=8/group) following 8-weeks of control conditions or treadmill Ex. Ex had no effect on body weight or blood pressure compared to respective controls. However, Ex prevented phenotypic whitening in OZR-Ex through elevated tPVAT nitric oxide production $(20 \%, \mathrm{p}<0.05)$. Additionally, OZR-Ex had lower tPVAT ROS production $(\mathrm{p}<0.01)$ measured by DHE and improved tPVAT 19S-dependent proteasome function measured by Ub4(lin)-GFP-35 degradation in LZR-Ex $(\mathrm{p}<0.05)$ and OZR-Ex $(\mathrm{p}<0.01)$. Improved tPVAT function resulted in a significantly $(\mathrm{p}<0.05)$ lower TNF $\alpha$, TSP-1, and higher IL-10 in OZR-Ex tPVAT. Whereas, LZR-Ex and OZR-Ex tPVAT had significantly $(\mathrm{p}<0.05)$ higher adiponectin levels compared to LZR and OZR controls. TPVAT increased LZR-Ex and OZR-Ex aortic nitric oxide and aortic relaxation. Finally, aortic stiffness (elastin modulus) was reduced in OZR-Ex compared to OZR controls. This was associated with increased TIMP-1 and decreased MMP-9 activity in the OZR-Ex tPVAT. Conclusion: Together these results suggest Ex prevents the phenotypic whitening of tPVAT associated with MetS. The change in OZR-Ex tPVAT cytokine profile is essential for the improved aortic relaxation and reduced aortic ROS following Ex. Finally, reduced MMP-9 activity and ROS contributed to prevention of aortic stiffness in OZREx. 


\section{Introduction:}

Metabolic Syndrome (MetS) increases cardiovascular mortality risk by 3-fold [1]. We and others have shown the MetS causes vascular dysfunction through increasing reactive oxygen species (ROS), inflammation, and reducing nitric oxide (NO) bioavailability [2-4]. An important cardiovascular risk factor associated with MetS is aortic stiffness, which increases the burden on the heart and reduces coronary profusion [5] and increases pulsatile flow leading to end organ damage [6].

Over the past decade, investigations into perivascular adipose tissue (PVAT) have uncovered its roles in regulating vascular function and the progression of vascular pathologies (chapter3)[7, 8]. We have recently shown PVAT surrounding the thoracic aorta (tPVAT) has an important role in mediating aortic endothelial dysfunction and structural stiffness in obese Zucker rats (OZR), a model of MetS. We identify TNF $\alpha$, as the key mediator of tPVAT dysfunction leading to the release of pro-inflammatory cytokines and the activation of aortic oxidative enzymes. We have also found impairment of proteasome function and a buildup of ubiquitin in MetS APVAT, which might exacerbate the oxidative and inflammatory environment within tPVAT.

It is well known aerobic exercise training (Ex) reduces the risk of cardiovascular disease, events, and mortality $[9,10]$. This is partially due to the restoration of NO a key mediator of aortic health and stiffness. Ex can improve NO through increasing endothelial NO synthase (eNOS) activation, expression [11, 12], levels of eNOS co-factors [13], decreasing the levels of inflammation [14], and reducing ROS [15]. Our previous clinical work has shown 8-weeks Ex reduces central pulse wave velocity, a clinical measure of aortic stiffness, in patients with MetS 
[16]. However, the effects of Ex on tPVAT and the role of tPVAT following Ex in mediating aortic function are unknown in MetS. Thus, we investigated the effect of Ex on tPVAT function, its subsequent regulation of aortic reactivity, and stiffness in the OZR. We hypothesize Ex will prevent the phenotypic shift of tPVAT in OZR and the subsequent tPVAT dysfunction. We further hypothesize the cytokine profile released from tPVAT following Ex will improve endothelial NO production and reduction of aortic stiffness. Finally, we hypothesize the changes in tPVAT are essential for Ex improvement of aortic function.

\section{Methods:}

Animals and Ex Intervention: Male lean (LZR, $n=16)$ and OZR $(n=16)$ were purchased from Envigo Laboratories at 7-9 weeks of age. Animals were housed at the West Virginia University Health Science Center (WVUHSC) AAALAC certified animal care facility on an approved protocol by the WVUHSC Animal Care and Use Committee. Animals received standard chow and tap water ad libitum. LZR and OZR were randomly assigned into control or Ex groups (LZR-Ex, n=8 \& OZR-Ex n=8). LZR-Ex and OZR-Ex underwent 8 weeks of treadmill running. Animals ran 5 days/week in individual lanes on a motor driven treadmill at a 5\% grade. During the first week, animals were acclimatized to the treadmill by progressively increasing running time form 20 minutes until a duration of 60 minutes was achieved. A maximum speed test was then performed on each animal and target running speed was set for $60-70 \%$ of that maximum. Workouts for the following 7 weeks were 60 minutes in duration and consisted of 15 minutes of gradual increases in speed until reaching target speed, which was maintained for remaining 45 minutes. Mild electrical stimulation was used to encourage running. Importantly, terminal procedures were performed a minimum of 48 hours following the last bout of Ex to eliminate the 
acute effects of exercise on experiments. At time of terminal procedures, animals were weighed then deeply anesthetized with pentobarbital sodium (50 mg/kg ip). All rats then received carotid artery and jugular vein cannulation to measure mean arterial pressure and to administer heparin, respectfully. The aorta with the surround tPVAT was removed and processed as previously published. Assessments of gene expression, tPVAT function, and aortic function were conducted as previously described in detail (Chapter 3). Hereafter experimental methods are briefly described.

Gene Expression: To assess gene expression, tPVAT was prepared and qrt-PCR carried out using the RNeasy Lipid Tissue Mini Kit (Qiagen) and Qiagen automated pipetting machines. 20 $\mu \mathrm{L}$ PCR reactions were mixed with QuantiTect primer assays and QuantiFast PCR master mix (Qiagen). Relative quantification was carried out by the $2^{\wedge}(\mathrm{ddCt})$ method with the reciprocal used for graphical representation of negative fold changes.

Measurement of ROS: Dihydroethidium (DHE, Invitrogen D1168) assays were performed on unfixed aortic rings and tPVAT sections placed in individual wells of a 96 well plate containing HEPES buffer with the following treatments: control (no added drug), tPVAT, Crossover OZR tPVAT (i.e., tPVAT from OZR on OZR-EX aortic ring or 4-Hydroxy-TEMPO (TEMPOL, $100 \mu \mathrm{M}$, Sigma-Aldrich 176141). Following completion of DHE incubation rings and PVAT were washed in HEPES buffer, placed separately in Optimal Cutting Temperature compound (OCT, Fisher Healthcare ${ }^{\mathrm{TM}}$ Tissue-Plus ${ }^{\mathrm{TM}}$ O.C.T Compound) frozen and then cut and stained/mounted with DAPI mounting media (VECTORSHEILD antifade mounting media with DAPI, Vector laboratories). Slides were imaged with an EVOS fluorescent microscope 
(Invitrogen EVOS FL Auto Cell Imaging System), three sections per image-treatment, were analyzed by Image J as fluorescent density/nucleus, the mean of the 3 images/treatment were used as the mean for each animal. Values were normalized to signal form TEMPOL treatment to eliminate background.

NO Bioavailability: Aortic rings were placed in individual wells of a 96 well plate containing HEPES buffer and 4-Amino-5-Methylamino-2',7'- Difluorofluorescein Diacetate (DAF-FM-DA,

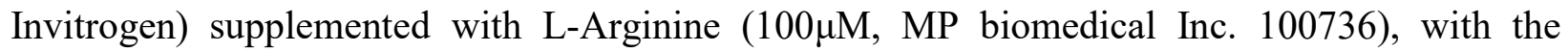
following treatments: control (no added drug), PVAT, Crossover PVAT, PVAT+TNF $\alpha$ AB $(4 \mu \mathrm{M})$ or nitro-L- arginine methyl ester (L-NAME, a potent inhibitor of NO synthase, SigmaAldrich N5751) and then stimulated with Acetyl- $\beta$-methylcholine chloride (methacholine (MCh), $1 \mathrm{X} 10^{-6}$, Sigma-Aldrich A2251). The conditioned solution was read in a plate reader excitation/emission at 495/515nm wavelength. Fluorescence was normalized to aorta length and L-NAME value.

tPVAT Cytokine Profile: tPVAT $(200 \mathrm{mg} / \mathrm{mL})$ was incubated in HEPES buffer for 1 hours at $37^{\circ} \mathrm{C}$. The tPVAT was then removed and the media was snap frozen and stored at $-80^{\circ} \mathrm{C}$. The conditioned media was then run on MSD multiplex rat inflammatory panel 2 (Mesoscale discovery, V-plex K15059D-2), MMP-9 activity ELISA (GE), and High molecular weight adiponectin ELISA (Mybiosource MBS020496). Additionally, tPVAT homogenates were prepared and run on MSD inflammation panel 1 rat (Mesoscale discovery, K15179C-9), 20S proteasome ELISA (Mybiosource MBS730715), and Ubiquitin ELISA (Mybiosource MBS039103). All assays were run per the manufacturer's instructions. Additionally, samples 
were run in standard western blot procedures to analyze the $19 \mathrm{~S}$ proteasome levels normalized to $\beta$-actin.

tPVAT Tissue Function: tPVAT homogenates were used to run SOD activity (Sigma-Aldrich 19160-1KT-F), total and phosphorylated NF-кB p65 (ThermoFisher multispecies InstantOne ${ }^{\mathrm{TM}}$ ELISA Kit, 85-86083-11). All assays were run per manufacturer's instructions. tPVAT homogenated in HEPES buffer containing 1mM DTT, 2mM ATP, and 10mM magnesium

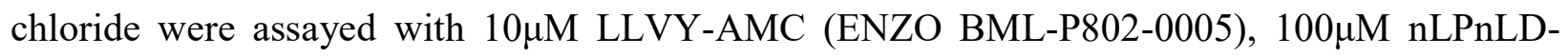

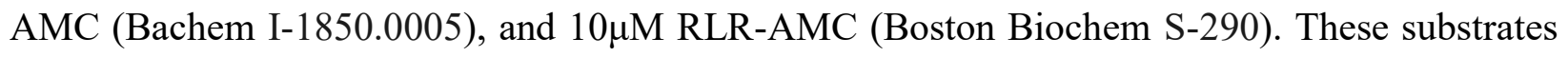
are cleaved by the three different protease sites in the $20 \mathrm{~S}$ core particle, and are thus a good general indicator of proteasome degradation capacity in the cell.

Proteasome assay was measured by kinetic read ( 1 read per minute for 120 minutes) on BioTek plate reader (ex. 380nm em. 460nm, BioTek Synergy HT) warmed to $37^{\circ} \mathrm{C}$. V-max for each assay was determined from 30 points on the linear portion of the kinetic read and normalized to V-max in the presence of the 20S inhibitor MG132 to account for background. Additionally, to directly test $19 \mathrm{~S}$ mediated proteasome function tPVAT homogenates were run in $\mathrm{Ub}_{4}$ (lin)-GFP$35(0.08 \mu \mathrm{g} /$ reaction $)$ degradation assays carried out in $50 \mu 1$ reactions using 96-half-well black plates (Corning) at $37^{\circ} \mathrm{C}$. GFP fluorescence was measured at every 60 seconds for 4 hours (ex/em:485/528). Degradation rates were determined in a similar manner to that described above. The Ub4 (lin)-GFP-35 substrate, a gift from Dr. David M. Smith, was generated as described by Martinez-Fonts and Matouschek [17]. 
Aortic Reactivity: Endothelial dependent dilation (EDD) and the effect of tPVAT on EDD were assessed in aortic rings cleaned and mounted in a myobath chamber between a fixed point and a force transducer (World Precision Instruments). The equilibrated aortic rings were constricted with phenylephrine and exposed to increasing doses of $\mathrm{MCh}\left(1 \mathrm{X} 10^{-9} \mathrm{M}\right.$ to $\left.1 \mathrm{X} 10^{-5} \mathrm{M}\right)$ and then increasing doses of the endothelial independent dilator sodium nitroprusside (SNP; 1 X10 $10^{-9} \mathrm{M}$ to $1 \mathrm{X} 10^{-5} \mathrm{M}$, MP Biomedical 152061).

Aortic Stiffness: Aortic rings were incubated in $\mathrm{Ca}^{2+}$ free Van Breemen solution to elicit a passive state. The rings were then mounted on an automated motorized force transducer (Aurora Scientific Inc. model $6350 * 358$ ) and force output was recorded in lab chart software by powerlab (AD instruments). After preconditioning rings were stretched to $10 \mathrm{mN}$ of force for 3 minutes during which time the internal diameter and wall thickness were measured. Subsequently, the automated force transducer increased the aortic ring diameter by $25 \%$ of initial internal diameter every 3 minutes until mechanical failure (determined by a drop in force following a stretch). Elastin modulus was determined as the slope of the stress-strain relationship.

Statistics: All data is represented as mean \pm standard error. All experiments were run in duplicate, at minimum, and the average of the reads used as the mean for each animal. Data analysis and graphing were conducted using GraphPad Prism 6 software (GraphPad Software, Inc.) and $\mathrm{p} \leq 0.05$ was set for statistical significance. Comparisons between groups were conducted using a one-way ANOVA, and a two-way repeated measure ANOVA for aortic function. 
$\underline{\text { Results: }}$

Animal characteristics: OZR present with obesity, increased MAP, fasting glucose and lipids compared to LZR. Importantly, for the analysis of our experimental data, no differences in BW, MAP and glucose were noted between the Ex and control groups (Table 4.1).

\section{tPVAT Environment}

tPVAT Phenotype: Disease states may alter adipocyte phenotype, which may be prevented by Ex. Indeed, compared to OZR controls, uncoupling protein-1 (UCP-1) expression was higher $(\mathrm{p}<0.01$, Fig.4.1A) in the OZR-Ex group. Similar, UCP-1 was higher in the LZR-Ex vs. LZR control group, suggesting a retention and strengthening of the brown-like phenotype, respectively. Further, our data showed OZR-Ex tPVAT had reduced expression of immunoattractant cytokines (Fig.4.1B) and lower gene expression of immune cell specific markers (Fig.4.1A). An important regulator of adipose phenotype is NO, which was reduced in OZRtPVAT. In contrast, NO bioavailability in tPVAT was higher in the OZR-Ex group vs. OZR control ( $p<0.05$, Fig.4.1C), and in LZR-Ex compared to LZR control ( $p<0.05$, Fig.4.1C). The changed NO bioavailability was not due to altered AKT activation, as phosphorylated AKT did not differ across the groups (Fig.4.1D).

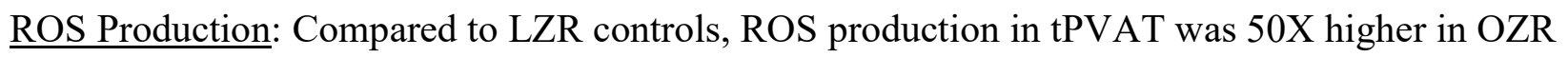
controls (Fig.4.1E); however, tPVAT ROS production was 95\% lower $(\mathrm{p}<0.05)$ in the OZR-Ex group compared to OZR controls, and not significantly different from LZR controls (Fig.4.1E). Whereas, no difference was noted in tPVAT ROS production between LZR controls and LZR- 
Ex. The lower ROS signal in OZR-Ex tPVAT was, in part, explained by a higher global SOD activity in the tPVAT from LZR-Ex and OZR-Ex vs. their respective controls ( $\mathrm{p}<0.05$, Fig.4.1F). Our previous data highlighted the essential role of tPVAT ROS production from NOX2 in mediating aortic dysfunction. Importantly, the gene expression of GP91 (the catalytic subunit of NOX2) was lower in LZR-Ex ( $<<0.05$, Fig.4.1D) and OZR-Ex ( $<<0.05$, Fig.4.1A) compared to the LZR and OZR controls. However, no differences in gene expression was noted between the Ex and control groups for the NOX2 regulatory subunit, p47phox. Other factors involved in ROS production were also affected by Ex, whereby the gene expression for SOD 1 and 2, NrF2, and GSR were higher in the OZR-Ex vs. OZR control group (Fig.1A, p<0.05). While only GSR was higher in LZR-Ex ( $<0.05$, Fig.4.1A). The oxidative load of cells is known to impact the ubiquitin-proteasome-system (UPS).

Proteasome Function: Our previous data (chapter 3) implicated proteasome dysfunction as a potential mechanism of tPVAT impairment in OZR. The LZR-Ex group had higher activity of the chymotrypsin-like active site and trypsin-like active site, whereas the OZR-Ex group had higher activity of the chymotrypsin-like active site and peptidylglutamyl-peptide hydrolyzing active site (Fig.4.2B) compared to their respective controls. Additionally, an increased expression of the $20 \mathrm{~S}$ catalytic core was noted in the OZR-Ex $(\mathrm{p}<0.05)$ but not in the LZR-Ex group (Fig.4.2A). The improved 20S core activity was accompanied by a lower expression of ubiquitin in both LZR-Ex and OZR-Ex compared to their respective controls ( $<<0.05$, Fig.4.2B). Ubiquitin degradation is dependent upon the 19S regulatory cap of the proteasome, so we assessed 19S-dependent degradation and 19S protein levels. The 19S dependent degradation rate of fluorescent ubiquitin was higher in the LZR-Ex and OZR-Ex groups compared to their 
respective controls ( $<<0.05$, Fig.4.2C). Further, 19S expression was significantly lower in the tPVAT of OZR control vs. LZR control. However, tPVAT 19S expression was higher in the OZR-Ex vs. OZR control ( $\mathrm{p}<0.01$, Fig.4.2E), and LZR controls ( $\mathrm{p}<0.05$, Fig.4.2E). Finally, a slightly higher 19S expression levels was noted in the LZR-Ex vs. LZR controls (Fig.4.2E). Thus far our data has shown the beneficial effects of Ex on tPVAT phenotype and function.

tPVAT cytokine production: ROS and ubiquitin can stimulate pro-inflammatory cytokines production. In tPVAT, LZR-Ex had similar anti- and pro-inflammatory cytokine profiles compared to LZR controls (Fig.4.3), however, a higher HMW adiponectin was noted in the LZREx tPVAT vs. LZR control ( $\mathrm{p}<0.05$, Fig.4.3B). OZR-Ex had increased (between 30-120\%, $\mathrm{p}<0.05$ ) levels of IL-4, IL-5, IL-10, IL-13 and HMW adiponectin released from the tPVAT compared to OZR controls (Fig.4.3A\&B). The gene expression of anti-inflammatory cytokines in the tPVAT were similar in both LZR-Ex and OZR-Ex compared to their respective controls (Fig.4.3C). Interestingly, OZR-Ex had 75\% and 25\% lower TNF $\alpha(p<0.01)$ and TSP-1 $(p<0.05)$ compared to OZR controls, respectively with no differences in tPVAT levels of IFN- $\gamma$ and IL-1 $\beta$ (Fig.4.3D\&E). Further, the only differences in the pro-inflammatory gene expression in the tPVAT were for TNF and IFN- $\gamma$, which were lower in the OZR-Ex vs. OZR control group (Fig.4.3F). These data suggest Ex prevented the increased release of TNF $\alpha$ from tPVAT associated with MetS, and increased IL-10 and restored HMW adiponectin. Thus, how would such changes impact the ROS production and EDD in the aorta?

tPVAT Regulation of Aortic Function 
Aortic ROS: Surprisingly, we found no differences on global aortic SOD activity in LZR-Ex and OZR-Ex compared to their respective controls (Fig.4.4A). However, in OZR-Ex, aortic ROS production was significantly lower vs. OZR controls (Fig.4.4B). Incubation of the OZR control aorta with OZR control tPVAT increased aortic ROS production, whereas incubating the OZREx aorta with OZR-Ex tPVAT further reduced aortic ROS production compared to OZR-Ex aorta with tPVAT incubation ( $\mathrm{p}<0.01$, Fig.4.4B). Aortic ROS production did not differ between LZR control and LZR-Ex (Fig.4.4B).

Aortic Reactivity: Similar to effects on ROS, aortic EDD was significantly higher in the OZR-Ex and LZR-Ex groups (without tPVAT) (Fig.4.4C). This was likely due to higher aortic NO production in the Ex groups, and the aortic NO production was further increased in the presence of tPVAT for both LZR-Ex and OZR-Ex (Fig.4.4D). The increased NO in the presence of tPVAT caused a further improvement in aortic EDD in both LZR-Ex and OZR-Ex (Fig.4.4F\&E).

tPVAT Crossover Experiments: With both tPVAT dependent and independent effects on aortic function improved following Ex we wanted to know if the aortic EDD in the OZR-Ex was protected against the detrimental effects of tPVAT from a OZR control. Incubation of the OZREx aorta with OZR control tPVAT reduced aortic EDD compared to OZR-Ex aorta with tPVAT $(\mathrm{p}<0.01$, Fig.4.5). However, if the OZR control tPVAT was treated with TNF $\alpha-n A B$, EDD was restored in the OZR-Ex aorta back to the same level as OZR-Ex aorta (without tPVAT) $(\mathrm{p}<0.05$, Fig.4.5). 
Aortic Structural Stiffness

Aortic stiffness in the OZR-Ex was lower compared to OZR controls $(p<0.05$, Fig.4.6A). Further, the tPVAT gene expression of MMP9 was lower in OZR-Ex vs. OZR controls, whereas no differences were noted in the gene expression of TIMP-1 (Fig.4.6B). In addition, MMP9 activity in TPVAT exudate was lower $(\mathrm{p}<0.05)$ in the OZR-Ex compared to OZR controls, and now similar to LZR controls (Fig.4.6D). Further, tPVAT TIMP-1 protein levels in OZR-Ex were reduced compared to OZR controls, and once again, similar to LZR controls (Fig.4.4C). Whereas, no differences were detected for MMP9 gene expression, MMP9 activity, TIMP-1 gene expression, or TIMP-1 protein levels in LZR-Ex vs. LZR controls (Fig.4.6B\&C).

\section{Discussion:}

For the first time, we evaluated the effects of Ex on MetS tPVAT and its regulation of aortic function. The present study presented novel data suggesting the detrimental impact of MetS on tPVAT and its impairment of aortic function was prevented by Ex. We show that 8-weeks of Ex had a significant impact on the tPVAT NO bioavailability and phenotype in OZR, accompanied by improvements in ROS, proteasome function, and inflammatory cytokine profile. Such significant changes essentially reversed the impact of MetS on tPVAT, with the subsequent improvement in aortic function, and a reduction in aortic stiffness. Additionally, our data suggests these tPVAT changes are essential for improved aortic function following Ex. 


\section{Effect of Exercise on Adipose Tissue}

tPVAT phenotype: Whitening adipose phenotypes are associated with increased oxidative stress and inflammation $[7,18,19]$. Pro-inflammatory cytokines have been linked to the suppression of UCP-1 [19], a hallmark of brown-like phenotype, exacerbating the whitening process. Additionally, sequestration of NO and eNOS uncoupling, are implicated in adipose dysfunction [20] and may contribute to the changing phenotype [21, 22]. Studies utilizing eNOS null mice highlight the importance of NO signaling for mitochondrial biogenesis, the brown adipose phenotype [21, 22], and the Ex induced browning of white adipose [22]. In the OZR, the increased oxidative load in PVAT likely interferes with NO bioavailability, leading to a drastic shift in tPVAT phenotype. In contrast, OZR-Ex had lower ROS production, a higher NO bioavailability, and a higher UCP-1 gene expression in tPVAT, suggesting a maintenance of the brown phenotype. Additionally, Ex may prevent eNOS uncoupling through an increase production of eNOS cofactors, as the expression levels of GCh1, the rate-limiting enzyme in $\mathrm{BH}_{4}$ production, was increased in the tPVAT from OZR-Ex. Taken together, these data suggest Ex promotes NO signaling in PVAT, which plays a pivotal role in establishing a healthy tPVAT phenotype in the OZR. The retention of the brown-like phenotype, in turn, supports an antioxidant tPVAT environment through UCP-1 signaling [18]. The direct mechanism driving the promotion of NO in tPVAT with Ex is unknown. However, it has been suggested that the myokine release from the exercising muscle [21] and autocrine signaling of adiponectin, which was higher in OZR-Ex, can promote adipose NO signaling and browning [23].

$\underline{\text { ROS: }}$ : Our data showed the tPVAT gene expression of pro-inflammatory immune cells was lower in the OZR-Ex vs. OZR controls. Additionally, Ex is known to alter immune polarization [24, 
25], thus reducing the oxidative and inflammatory burden, of immune cells. Reduced immune cell populations in tPVAT likely contributes to the reduction of the NOX2 expression [26]. In contrast, the IPVAT gene expression of the p47phox regulatory subunit was similar in the OZREx and OZR controls, likely reflecting differential regulation between gene expression, protein levels, and activation [27] or p47phox mediation of important cellular function independent of the NOX compound [28]. Our previous work showed the NOX2 enzyme was a major culprit of ROS production in OZR tPVAT (REF). This would suggest a reduction in tPVAT ROS production was the result of Ex limiting the tPVAT phenotypic shift and NOX2 expressing immune cell infiltration, coupled with an increase in SOD activity.

Proteasome Function: The high oxidative load in OZR tPVAT may be exacerbated by the buildup of ubiquitin due to reduced proteasome function. The buildup of ubiquitin can lead to increased inflammatory cytokine production and ROS associated with MetS [29-31]. Ex has been shown to improve proteasome function in muscle [32] but the effect of Ex on the UPS in tPVAT had not been previously explored. We show Ex reduced ubiquitin and increased activity of the proteasome, with the largest effect on increasing 19S Cap expression thus facilitating improvements in substrate [33] recognition and 20S gate opening [34]. This was supported by our data, which showed increased rate of substrate breakdown (Fig.2). It has been speculated higher oxidative environments, like in OZR tPVAT, may cause the 19S cap to dissociate from the 20S core reducing the ability to recognize and degrade ubiquitin [35]. This was supported by the reduced expression of the 19S cap and ubiquitin buildup in OZR-tPVAT. This suggests the increase of the 19S cap expression in OZR-Ex is partly mediated through lower ROS production. Proteasome function in OZR-Ex may be additionally improved through increased 20S core 
proteasome concentration, which may serve as a compensatory adaptation to Ex, increasing proteasome number to account for the diminished function in the OZR control tPVAT. Taken together, Ex significantly increased proteasome concentration of both the 19S regulatory cap and the $20 \mathrm{~S}$ core. This culminates in greatly improved recognition and breakdown of ubiquitin, as supported by the $\mathrm{Ub}_{4}$ (lin)-GFP-35 substrate assay, ultimately reducing the ubiquitin stimulation of inflammatory cytokines following Ex in MetS. Uncovering the adipose signaling pathways underlying the maintenance or alteration in proteasome function needs further investigation.

tPVAT Cytokines: In our study, the OZR-Ex group had reductions in two known inflammatory cytokine signaling pathways; 1) ubiquitin [29, 30] and, 2) ROS [36]. We have previously shown the IPVAT ROS production, and IPVAT mediated aortic impairment in the OZR was dependent on TNF $\alpha$ (chapter3). Importantly, the OZR-Ex had a drastically lower production of TNF $\alpha$ from the tPVAT, and higher IL-10 and HMW adiponectin vs. OZR controls. IL-10 is a known inhibitor of TNF $\alpha$ [37] and ROS [38]. The lower production of TNF $\alpha$ and immune cell population in OZR-Ex tPVAT likely has 3 important actions on the autocrine signaling of tPVAT. First, reducing TNF $\alpha$ removes autocrine activation of tPVAT ROS, which subsequently decreases oxidative dissociation of the 19S from the proteasome improving function, decreases sequestration of NO, and decreases activation of transcription factors. Secondly, reducing TNF $\alpha$ removes the inhibition on HMW adiponectin [39], allowing for autocrine activation of NO bioavailability and the promotion of the brown-like phenotype. Lastly, reducing TNF $\alpha$ would downregulate TSP-1 (Fig.3), which would remove TSP-1 inhibition of eNOS function and antiangiogenic actions, a potentially cause of obesity related adipose dysfunction [40, 41]. These actions of Ex on cytokine profile would have important implications on the tPVAT mediated 
function of the aorta $[39,42]$.

\section{Role of tPVAT on Aortic Function}

Aortic Function: Aortic ROS was drastically lower in OZR-Ex coupled with a higher aortic NO bioavailability and a higher EDD compared to OZR controls despite no changes in body weight or MAP with Ex. The improved aortic EDD occurred despite a lack of effect on global SOD activity in the OZR-Ex and LZR-Ex groups. Other studies clearly show vascular SOD levels are increased following Ex $[43,44]$. The lack of difference in SOD activity in our study may reflect differences in Ex protocol, protein level vs. activity, or age of the animals [15]. Additionally, SOD-1 is the focus of many previous reports, so SOD-1 changes may be masked by no Ex on other isoforms [44] in our assessment of global SOD activity. In addition to the reduced basal ROS production in the OZR-EX, incubation of the OZR aorta with OZR-Ex tPVAT actively suppressed aortic ROS production.

tPVAT Mediated Aortic Function: Improved tPVAT mediated EDD was the result of increased NO in LZR-Ex and OZR-Ex and inhibition of ROS in OZR-Ex. The tPVAT inhibition of aortic ROS production is likely due to the higher levels of IL-10 inhibiting NOX [38] a significant contributor to aortic ROS production [45] (paper 1). Additionally, the reduced secretion of TNF $\alpha$ removes the stimulus for NOX activation contributing to reduced aortic ROS. This suggests Ex prevents the shift towards ROS activators and away from ROS inhibitors associated with MetS. Increased NO is likely due to the actions of the increased secretion of HMW adiponectin [39] and the decreased TSP-1 (in OZR-EX) [46]. These data again highlight the importance of tPVAT TNF $\alpha$ - IL-10- HMW adiponectin balance, this time in effecting aortic function. These data 
strongly suggest changes in TPVAT cytokine release as a major pathway of Ex aortic adaptation. This idea is supported by our crossover experiments, in which OZR-Ex aorta was not protected against the inflammatory insult released from OZR-tPVAT, chiefly TNF $\alpha$. Suggesting the improved aortic function following Ex is dependent on reduced inflammatory cytokine release from tPVAT. Future studies are needed to assess the effect of Ex in other PVAT depots and vascular beds. Understanding key cellular events in PVAT in response to Ex might uncover a therapeutic target to prevent the progression of vascular pathologies.

To date only one other study, to our knowledge, has examined the effect of Ex on PVAT regulation of vascular function. In healthy rats, exercise had no impact on PVAT regulation of aortic function [47]. Similarly, we found very few changes in tPVAT between LZR and LZR-Ex outside of increased HMW adiponectin release and improved EDD. The difference in adiponectin findings might arise from measuring tissue vs. secreted levels and measuring adiponectin vs. HMW adiponectin. Additionally, differences may have arisen from the different rat strains used and/or differences in total work. This may suggest the exercise stimulus was greater in the current study due to the incorporation of vertical work utilizing $5 \%$ incline compared to $0 \%$.

Aortic Stiffness and tPVAT: Finally, we showed Ex prevented the structural stiffening of the aorta associated with MetS. This is presumably due to Ex reduction of aortic ROS, in part, mediated by the altered tPVAT cytokine profile. Additionally, Ex preserved TIMP-1 levels and reduced MMP-9 activity in the tPVAT. Both ROS and MMP-9 are known to cause elastin fragmentation [8, 48, 49], which leads to reduced distensibility [50]. Ex reduction of TNF $\alpha$ and 
TSP-1, known activators of MMP-9 [51, 52], may explain the lower MMP-9 activity in tPVAT. These findings may help to explain results from a previous study in patients with MetS, in which we found Ex reduced aortic pulse wave velocity (a clinical measure of aortic stiffness) independent of changes in circulating inflammation and markers of oxidative stress [16]. Our results from the current study suggest changes in tPVAT of MetS patients might have been responsible for the observed improvement in pulse wave velocity.

Limitations: One limitation to our current study is Ex was introduced during the development of MetS. Thus, these data should be interpreted as such and future studies to determine the therapeutic efficacy of Ex after the development of MetS on tPVAT function and regulation of the aortic endothelium are needed. However, we have previously shown that the OZR by 9 weeks of age already exhibits some vascular impairment and alterations in circulating cytokines [2]. Suggesting our data might then represent a halt in the progression of MetS induced vascular pathology and a reversal in the impairments present at 9 weeks of age. Additionally, small n's were utilized in gene expression experiments due to limited LZR tPVAT. However, many genes showed robust and consistent responses such as UCP-1 and TNF. Finally, only global SOD activity was measured, and data may not reflect regional alterations in specific isoforms.

\section{Conclusions and Prospective}

The present study is the first examination of the Ex effect on tPVAT and its regulation of aortic function in MetS. The promotion of physical activity lifestyle even independent of weight loss and changes in blood pressure may prevent the accelerated aortic impairment seen with obesity and MetS through preservation of tPVAT NO signaling and brown-like phenotype. Ex prevented 
the increase of ROS and inflammation associated with MetS, while enhancing proteasome function in tPVAT. Additionally, our data suggests, at least, in part the aortic adaptations to Ex are dependent on the function of the tPVAT. This was supported by the lack of protection in OZR-Ex aortas against OZR tPVAT impairment of NO and EDD. This suggests therapeutically targeting tPVAT in combination with Ex or other vascular therapeutics in patients with MetS might accelerate beneficial vascular adaptation and reduce the cardiovascular burden of obesity.

\section{References:}

1. Grundy, S.M., et al., Diagnosis and management of the metabolic syndrome: an American Heart Association/National Heart, Lung, and Blood Institute scientific statement. Curr Opin Cardiol, 2006. 21(1): p. 1-6.

2. Brooks, S.D., et al., Metabolic syndrome impairs reactivity and wall mechanics of cerebral resistance arteries in obese Zucker rats. Am J Physiol Heart Circ Physiol, 2015. 309(11): p. H1846-59.

3. Fortuno, A., et al., Phagocytic NADPH oxidase overactivity underlies oxidative stress in metabolic syndrome. Diabetes, 2006. 55(1): p. 209-15.

4. Roberts, C.K., et al., Oxidative stress and dysregulation of $N A D(P) H$ oxidase and antioxidant enzymes in diet-induced metabolic syndrome. Metabolism, 2006. 55(7): p. 928-34.

5. Mattace-Raso, F.U., et al., Arterial stiffness and risk of coronary heart disease and stroke: the Rotterdam Study. Circulation, 2006. 113(5): p. 657-63. 
6. Saji, N., et al., Association between silent brain infarct and arterial stiffness indicated by brachial-ankle pulse wave velocity. Intern Med, 2012. 51(9): p. 1003-8.

7. Brown, N.K., et al., Perivascular adipose tissue in vascular function and disease: a review of current research and animal models. Arterioscler Thromb Vasc Biol, 2014. 34(8): p. 1621-30.

8. Fleenor, B.S., et al., Superoxide signaling in perivascular adipose tissue promotes agerelated artery stiffness. Aging Cell, 2014. 13(3): p. 576-8.

9. Cornelissen, V.A. and R.H. Fagard, Effects of endurance training on blood pressure, blood pressure-regulating mechanisms, and cardiovascular risk factors. Hypertension, 2005. 46(4): p. 667-75.

10. Miller, T.D., G.J. Balady, and G.F. Fletcher, Exercise and its role in the prevention and rehabilitation of cardiovascular disease. Ann Behav Med, 1997. 19(3): p. 220-9.

11. Hambrecht, R., et al., Regular physical activity improves endothelial function in patients with coronary artery disease by increasing phosphorylation of endothelial nitric oxide synthase. Circulation, 2003. 107(25): p. 3152-8.

12. Kojda, G., et al., Dysfunctional regulation of endothelial nitric oxide synthase (eNOS) expression in response to exercise in mice lacking one eNOS gene. Circulation, 2001. 103(23): p. 2839-44.

13. Sindler, A.L., et al., Effects of ageing and exercise training on eNOS uncoupling in skeletal muscle resistance arterioles. J Physiol, 2009. 587(Pt 15): p. 3885-97.

14. Drenth, J.P., et al., Endurance run increases circulating IL-6 and IL-1ra but downregulates ex vivo TNF-alpha and IL-1 beta production. J Appl Physiol (1985), 1995. 79(5): p. 1497-503. 
15. Durrant, J.R., et al., Voluntary wheel running restores endothelial function in conduit arteries of old mice: direct evidence for reduced oxidative stress, increased superoxide dismutase activity and down-regulation of NADPH oxidase. J Physiol, 2009. 587(Pt 13): p. 3271-85.

16. Donley, D.A., et al., Aerobic exercise training reduces arterial stiffness in metabolic syndrome. J Appl Physiol (1985), 2014. 116(11): p. 1396-404.

17. Martinez-Fonts, K. and A. Matouschek, A Rapid and Versatile Method for Generating Proteins with Defined Ubiquitin Chains. Biochemistry, 2016. 55(12): p. 1898-908.

18. Lin, Y., et al., The hyperglycemia-induced inflammatory response in adipocytes: the role of reactive oxygen species. J Biol Chem, 2005. 280(6): p. 4617-26.

19. Sakamoto, T., et al., Inflammation induced by RAW macrophages suppresses UCP1 mRNA induction via ERK activation in 10T1/2 adipocytes. Am J Physiol Cell Physiol, 2013. 304(8): p. C729-38.

20. Xia, N., et al., Uncoupling of Endothelial Nitric Oxide Synthase in Perivascular Adipose Tissue of Diet-Induced Obese Mice. Arterioscler Thromb Vasc Biol, 2016. 36(1): p. 7885.

21. Bostrom, P., et al., A PGCl-alpha-dependent myokine that drives brown-fat-like development of white fat and thermogenesis. Nature, 2012. 481(7382): p. 463-8.

22. Trevellin, E., et al., Exercise training induces mitochondrial biogenesis and glucose uptake in subcutaneous adipose tissue through eNOS-dependent mechanisms. Diabetes, 2014. 63(8): p. 2800-11.

23. Koh, E.H., et al., eNOS plays a major role in adiponectin synthesis in adipocytes. Am J Physiol Endocrinol Metab, 2010. 298(4): p. E846-53. 
24. Kawanishi, N., et al., Exercise training inhibits inflammation in adipose tissue via both suppression of macrophage infiltration and acceleration of phenotypic switching from M1 to M2 macrophages in high-fat-diet-induced obese mice. Exerc Immunol Rev, 2010. 16: p. $105-18$.

25. Oliveira, A.G., et al., Acute exercise induces a phenotypic switch in adipose tissue macrophage polarization in diet-induced obese rats. Obesity (Silver Spring), 2013. 21(12): p. 2545-56.

26. Bedard, K. and K.H. Krause, The NOX family of ROS-generating NADPH oxidases: physiology and pathophysiology. Physiol Rev, 2007. 87(1): p. 245-313.

27. Fan, L.M., L. Teng, and J.M. Li, Knockout of $p 47$ phox uncovers a critical role of $p 40$ phox in reactive oxygen species production in microvascular endothelial cells. Arterioscler Thromb Vasc Biol, 2009. 29(10): p. 1651-6.

28. Montezano, A.C. and R.M. Touyz, Mechanosensitive regulation of cortactin by p47phox: a new paradigm in cytoskeletal remodeling. Circ Res, 2013. 112(12): p. 1522-5.

29. Ghosh, A.K., et al., Elevated Endoplasmic Reticulum Stress Response Contributes to Adipose Tissue Inflammation in Aging. J Gerontol A Biol Sci Med Sci, 2015. 70(11): p. 1320-9.

30. Hohn, A., J. Konig, and T. Jung, Metabolic Syndrome, Redox State, and the Proteasomal System. Antioxid Redox Signal, 2016. 25(16): p. 902-917.

31. Otoda, T., et al., Proteasome dysfunction mediates obesity-induced endoplasmic reticulum stress and insulin resistance in the liver. Diabetes, 2013. 62(3): p. 811-24. 
32. Cunha, T.F., et al., Aerobic exercise training upregulates skeletal muscle calpain and ubiquitin-proteasome systems in healthy mice. J Appl Physiol (1985), 2012. 112(11): p. 1839-46.

33. Ehlinger, A. and K.J. Walters, Structural insights into proteasome activation by the $19 S$ regulatory particle. Biochemistry, 2013. 52(21): p. 3618-28.

34. Rabl, J., et al., Mechanism of gate opening in the 20S proteasome by the proteasomal ATPases. Mol Cell, 2008. 30(3): p. 360-8.

35. Wang, X., et al., Regulation of the $26 \mathrm{~S}$ proteasome complex during oxidative stress. Sci Signal, 2010. 3(151): p. ra88.

36. Gloire, G., S. Legrand-Poels, and J. Piette, NF-kappaB activation by reactive oxygen species: fifteen years later. Biochem Pharmacol, 2006. 72(11): p. 1493-505.

37. Zemse, S.M., et al., Interleukin-10 inhibits the in vivo and in vitro adverse effects of TNFalpha on the endothelium of murine aorta. Am J Physiol Heart Circ Physiol, 2010. 299(4): p. H1160-7.

38. Kamizato, M., et al., Interleukin 10 inhibits interferon gamma- and tumor necrosis factor alpha-stimulated activation of NADPH oxidase 1 in human colonic epithelial cells and the mouse colon. J Gastroenterol, 2009. 44(12): p. 1172-84.

39. Wang, Z.V. and P.E. Scherer, Adiponectin, cardiovascular function, and hypertension. Hypertension, 2008. 51(1): p. 8-14.

40. Rausch, M.E., et al., Obesity in C57BL/6J mice is characterized by adipose tissue hypoxia and cytotoxic T-cell infiltration. Int J Obes (Lond), 2008. 32(3): p. 451-63.

41. O'Rourke, R.W., et al., Hypoxia-induced inflammatory cytokine secretion in human adipose tissue stromovascular cells. Diabetologia, 2011. 54(6): p. 1480-90. 
42. Didion, S.P., et al., Endogenous interleukin-10 inhibits angiotensin II-induced vascular dysfunction. Hypertension, 2009. 54(3): p. 619-24.

43. de Moraes, C., et al., Exercise training improves relaxation response and SOD-1 expression in aortic and mesenteric rings from high caloric diet-fed rats. BMC Physiol, 2008. 8: p. 12.

44. Rush, J.W., J.R. Turk, and M.H. Laughlin, Exercise training regulates SOD-1 and oxidative stress in porcine aortic endothelium. Am J Physiol Heart Circ Physiol, 2003. 284(4): p. H1378-87.

45. Yang, B. and V. Rizzo, TNF-alpha potentiates protein-tyrosine nitration through activation of NADPH oxidase and eNOS localized in membrane rafts and caveolae of bovine aortic endothelial cells. Am J Physiol Heart Circ Physiol, 2007. 292(2): p. H95462.

46. Isenberg, J.S., et al., Regulation of nitric oxide signalling by thrombospondin 1: implications for anti-angiogenic therapies. Nat Rev Cancer, 2009. 9(3): p. 182-94.

47. Araujo, H.N., et al., Perivascular adipose tissue and vascular responses in healthy trained rats. Life Sci, 2015. 125: p. 79-87.

48. Chen, J.Y., et al., Increased aortic stiffness and attenuated lysyl oxidase activity in obesity. Arterioscler Thromb Vasc Biol, 2013. 33(4): p. 839-46.

49. Yasmin, et al., Matrix metalloproteinase-9 (MMP-9), MMP-2, and serum elastase activity are associated with systolic hypertension and arterial stiffness. Arterioscler Thromb Vasc Biol, 2005. 25(2): p. 372. 
50. Lakatta, E.G. and D. Levy, Arterial and cardiac aging: major shareholders in cardiovascular disease enterprises: Part I: aging arteries: a "set up" for vascular disease. Circulation, 2003. 107(1): p. 139-46.

51. Donnini, S., et al., ERK1-2 and p38 MAPK regulate MMP/TIMP balance and function in response to thrombospondin-1 fragments in the microvascular endothelium. Life Sci, 2004. 74(24): p. 2975-85.

52. Wu, H.T., et al., Identifying the regulative role of $N F$-kappaB binding sites within promoter region of human matrix metalloproteinase 9 (mmp-9) by TNF-alpha induction. Appl Biochem Biotechnol, 2013. 169(2): p. 438-49. 
Figure Legends

Figure. 4.1 tPVAT Reactive Oxygen Species and Phenotype. A) tPVAT relative gene expression compared to LZR control for markers of phenotype, immune cell markers, and pro/ antioxidants $(n=3)$, while B) represents protein levels of immune-attractants measured in tPVAT exudate $(n=5)$. C) tPVAT production of NO measured by fluorescent DAF-FM (n=8) and D) highlights activation of the AKT pathway $(n=5)$. Finally, E\&F) represent tPVAT DHE measured ROS production and total SOD activity $(n=5-8)$. Data are represented as Mean \pm SEM *denotes statistical difference compared to LZR control, ${ }^{\#}$ denotes statistical difference compared to OZR control measured by ANOVA with Tukey Post-Hoc, $\mathrm{p}<0.05$. UCP-1, uncoupling protein-1; CD, cluster of differentiation; eNOS, endothelial nitric oxide synthase; Gch, GTP cyclohydrolase; SOD, superoxide dismutase; NOX2, NADPH oxidase 2 catalytic subunit (GP91); p47phox, NADPH oxidase 2 intracellular regulatory subunit; Keap1, kelch-like ECH associated protein 1, GSR, glutathione reductase; CAT, catalase, $\mathrm{KC} / \mathrm{GRO}$, chemokine (C-C motif) ligand 1; MCP-1, monocyte chemoattractant protein-1; p-AKT, phosphorylated- Protein Kinase B.

Figure. 4.2 Ex Enhancement of Proteasome Function. A) Proteasome function measured across all 3 active sites ( $n=6-8)$; LLVY (chymotrypsin-like), RLR (trypsin-like) and nLPnLD (peptidylglutamyl-peptide hydrolyzing) and B) levels of ubiquitin and the 20S proteasome from tPVAT homogenates $(n=5)$. C) Depiction of $19 \mathrm{~S}$ dependent regulation of fluorescent ubiquitin degradation $(n=6), D \& E)$ representative western blot of $19 S$ protein levels and quantification $(\mathrm{n}=4)$. Data are represented as Mean \pm SEM *denotes statistical difference compared to LZR control, \#denotes statistical difference compared to OZR control measured by ANOVA with Tukey Post-Hoc, $\mathrm{p}<0.05$. 
Figure. 4.3 tPVAT Cytokine Profile Following Ex. A\&B) Anti-inflammatory cytokine levels measured in tPVAT exudate $(n=5)$ with B) supporting tPVAT relative gene expression $(n=3)$. D) Pro-inflammatory cytokine levels measured in PVAT exudate $(\mathrm{n}=5)$ and E) tissue homogenates $(n=5)$ with F) relative gene expression of inflammatory genes $(n=3)$. Data are represented as Mean \pm SEM *denotes statistical difference compared to LZR control, \#denotes statistical difference compared to OZR control measured by ANOVA with Tukey Post-Hoc, $\mathrm{p}<0.05$. IL, interleukin; HMW adiponectin, high molecular weight adiponectin; TNF $\alpha$, tumor necrosis factor alpha; IFN- $\gamma$, interferon gamma; TSP-1, thrombospondin 1; CCL, Chemokine (c-c motif) ligand; CCR, Chemokine (c-c motif) receptor.

Figure. 4.4 Effect of Ex on tPVAT Mediated Aortic Function. A\&B) Relative total aortic SOD activity $(n=5)$ and aortic ROS production with and without tPVAT incubation $(n=8)$. C) Effect of Ex on aortic $\operatorname{EDD}(n=8)$ and D) NO production with and without tPVAT incubation $(n=8)$. Ex effect on tPVAT mediated aortic EDD in E) OZRs $(n=8)$ and F) LZRs $(n=8)$. Data are represented as Mean \pm SEM *denotes statistical difference compared to LZR control, "denotes statistical difference compared to OZR control, ^denotes statistical significant effect of tPVAT measured by ANOVA in Panel A and repeated measures ANOVA in B-F both with Tukey PostHoc, $\mathrm{p}<0.05$. SOD, Superoxide dismutase.

Figure. 4.5 Ex Does Not Protect the Aorta Against tPVAT Derived TNF $\alpha$ Mediated Impairment. The graph depicts OZR-Ex aortic EDD when crossover treated with OZR tPVAT $(n=8)$ and with OZR tPVAT+TNF $\alpha-n A B(n=5)$. Data are represented as Mean \pm SEM ^denotes 
statistically significant effect of crossover OZR tPVAT compared to OZR-EX tPVAT, ${ }^{\$}$ denotes statistically significant effect of TNF $\alpha-n A B$ treatment compared to the OZR tPVAT crossover measured by repeated measures ANOVA with Tukey Post-Hoc, $\mathrm{p}<0.05$. TNF $\alpha-\mathrm{nAB}$, tumor necrosis factor alpha- neutralizing anti-body.

Figure. 4.6 Ex Prevents Aortic Stiffness. A) Aortic stiffness measured by elastin modulus in aortic rings $(\mathrm{n}=8)$. B) Remodeling factor gene expression $(\mathrm{n}=3)$, C) tPVAT TIMP-1 protein levels ( $\mathrm{n}=5)$, and D) MMP-9 activity in tPVAT exudate $(\mathrm{n}=5)$. Data are represented as Mean \pm SEM *denotes statistical difference compared to LZR control, "denotes statistical difference compared to OZR control measured by ANOVA with Tukey Post-Hoc, $p<0.05$. MMP, matrix metalloproteinase; TIMP, tissue inhibitor of metalloproteinase. 
Table 4.1. Body Weight, Blood Pressure, and Blood Profile of LZR and OZR

\begin{tabular}{|l|l|l|l|l|}
\hline Table 1 & \multicolumn{2}{|c|}{ LZR } & \multicolumn{2}{c|}{ OZR } \\
\hline & Control & Exercise & Control & Exercise \\
\hline Body Mass (g) & $418 \pm 17$ & $363 \pm 11$ & $604 \pm 10^{*}$ & $627 \pm 19$ \\
\hline MAP (mmHg) & $106 \pm 3$ & $114 \pm 2$ & $135 \pm 6^{*}$ & $134 \pm 6$ \\
\hline Glucose (mg/dl) & $98 \pm 6$ & $101 \pm 8$ & $184 \pm 12^{*}$ & $154 \pm 12$ \\
\hline TG (mg/dl) & $25 \pm 3$ & $31 \pm 3$ & $124 \pm 8^{*}$ & $82 \pm 15 \#$ \\
\hline
\end{tabular}

Data represented as Mean \pm SEM, ${ }^{*}$ denotes statistical difference compared to LZR control and \# denotes statistical difference from OZR control measured by ANOVA with Tukey Post-Hoc $\mathrm{p}<0.05(\mathrm{n}=6-8)$. MAP, mean arterial pressure; TG, triglyceride 
Figures

Figure 4.1. tPVAT Reactive Oxygen Species and Phenotype
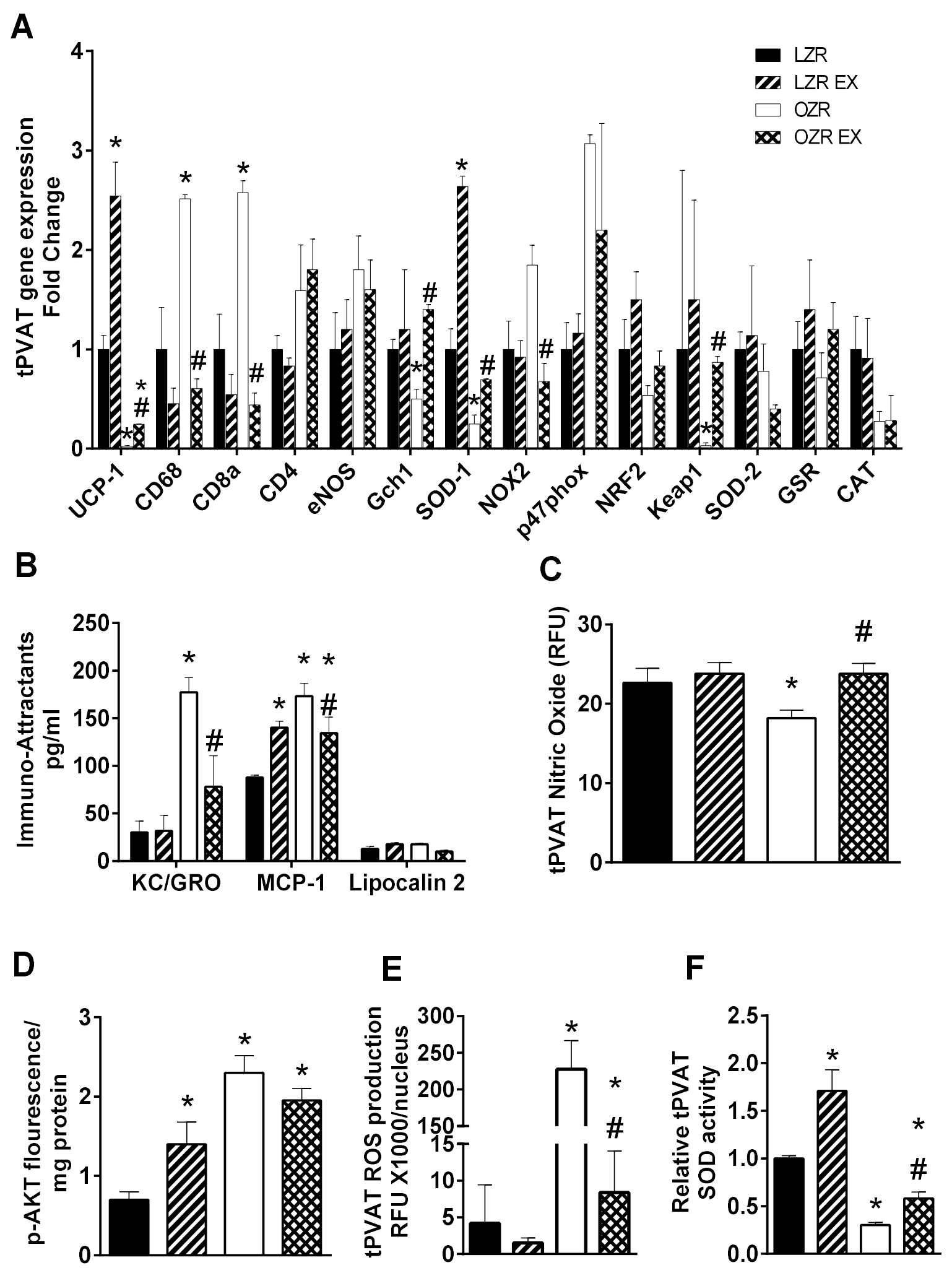
Figure 4.2. Ex Enhancement of Proteasome Function
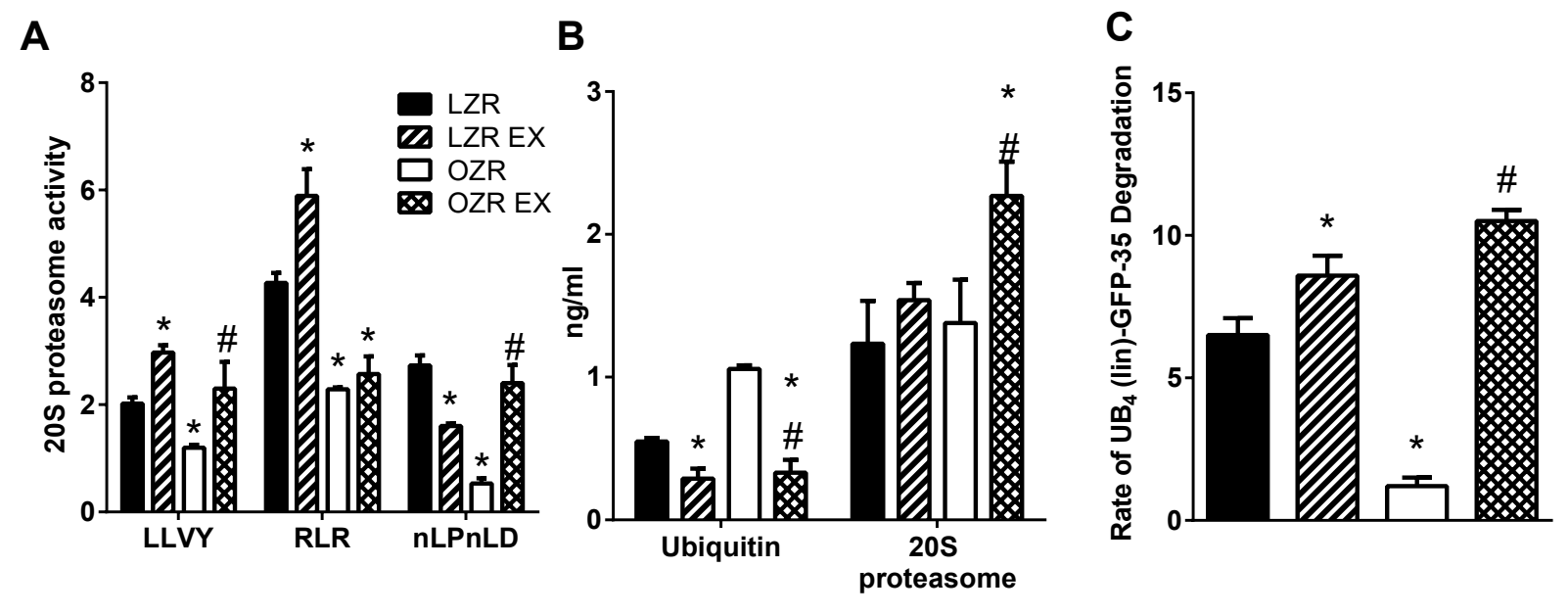

D

E
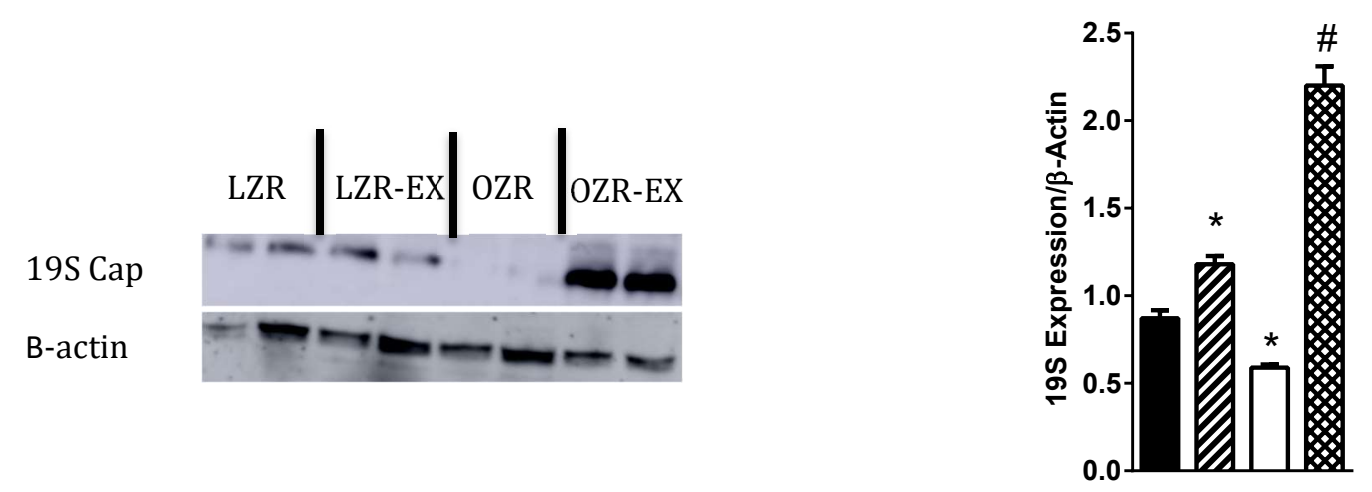
Figure 4.3. tPVAT Cytokine Profile Following Ex

A

B

C
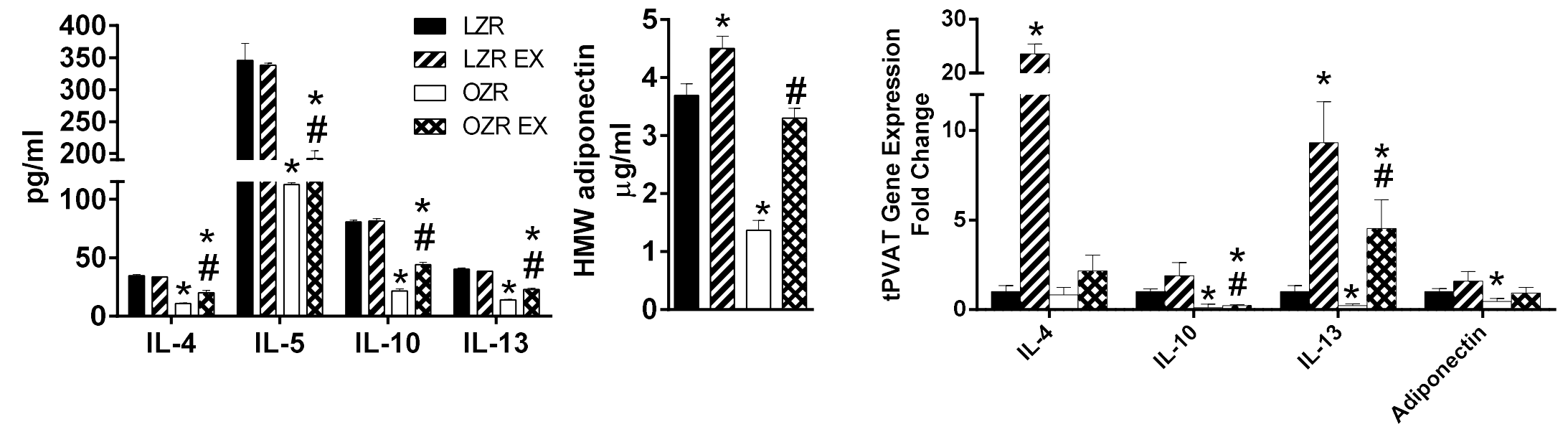

D

\section{E}

F

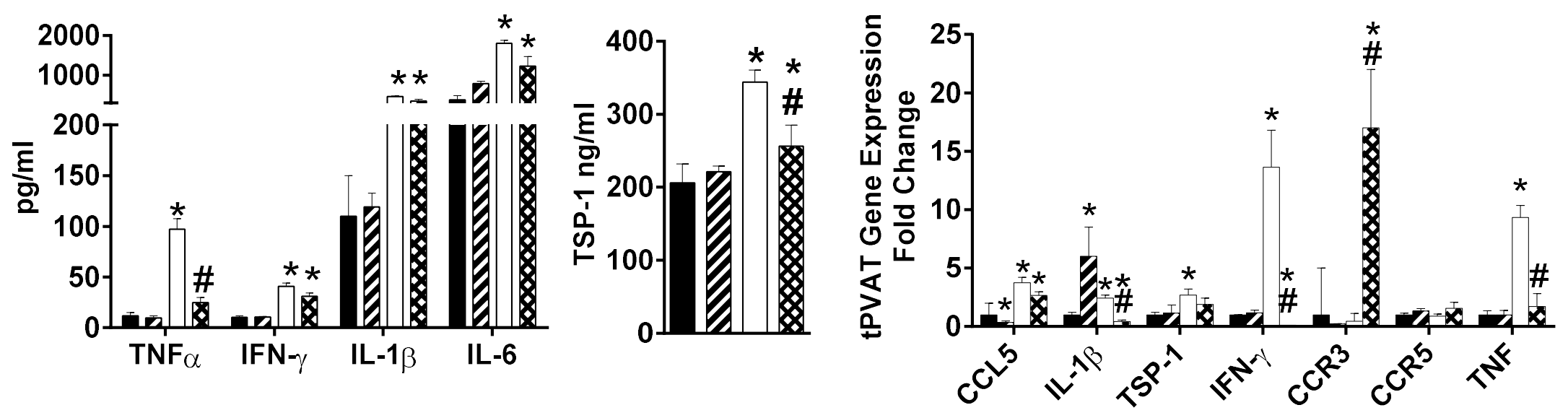


Figure 4.4. Effect of Ex on tPVAT Mediated Aortic Function

A

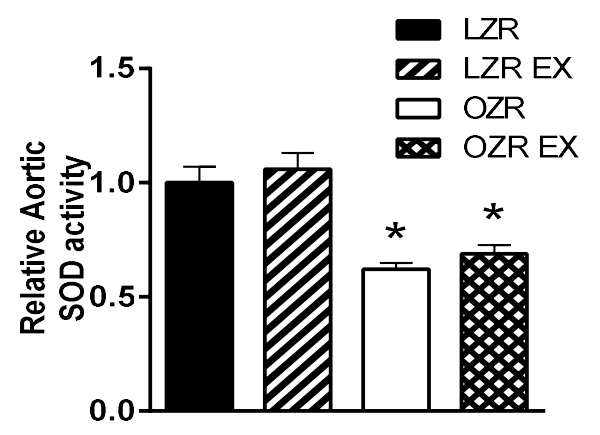

B

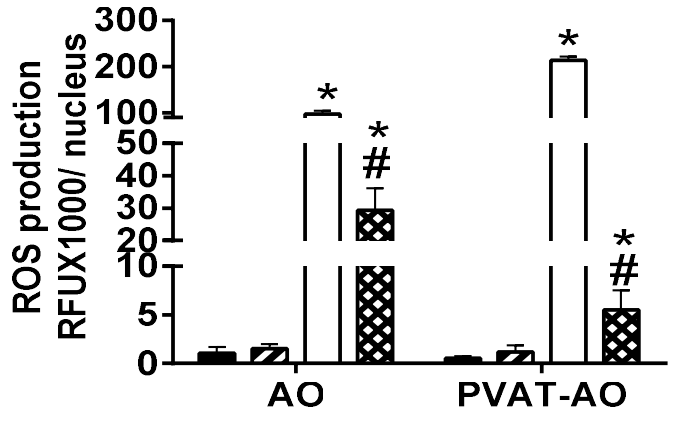

E

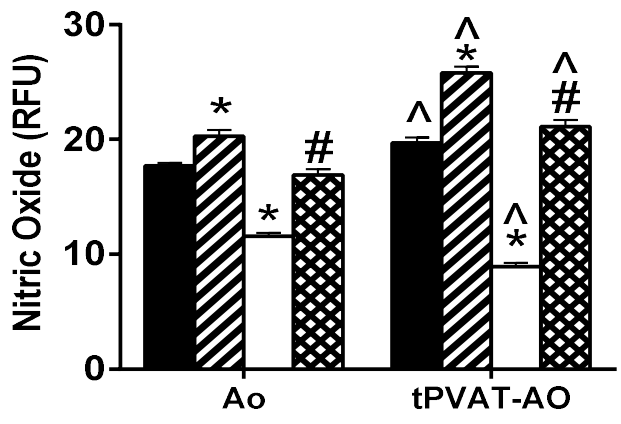

C

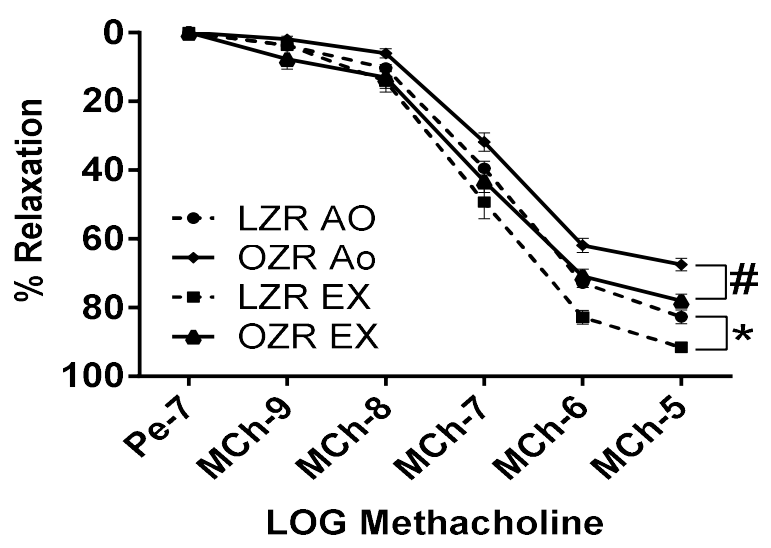

$\mathbf{F}$

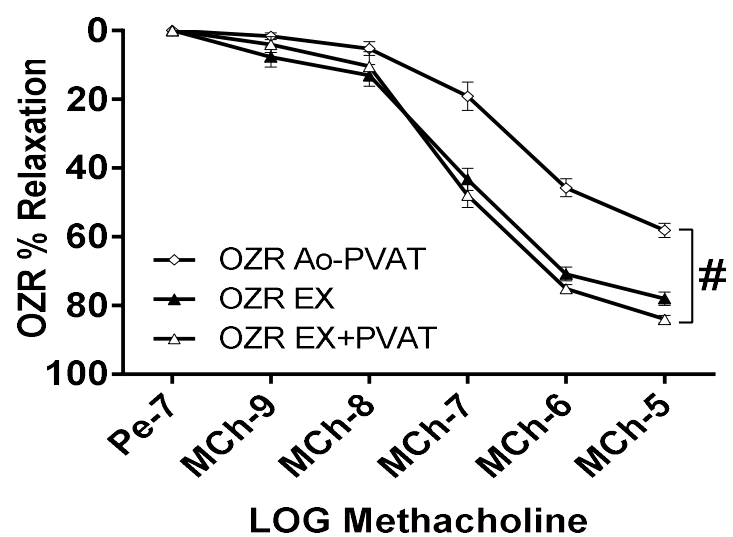

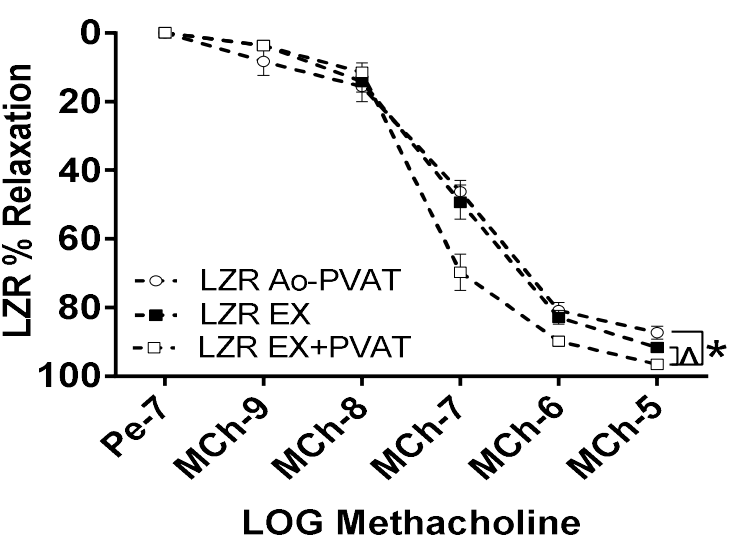




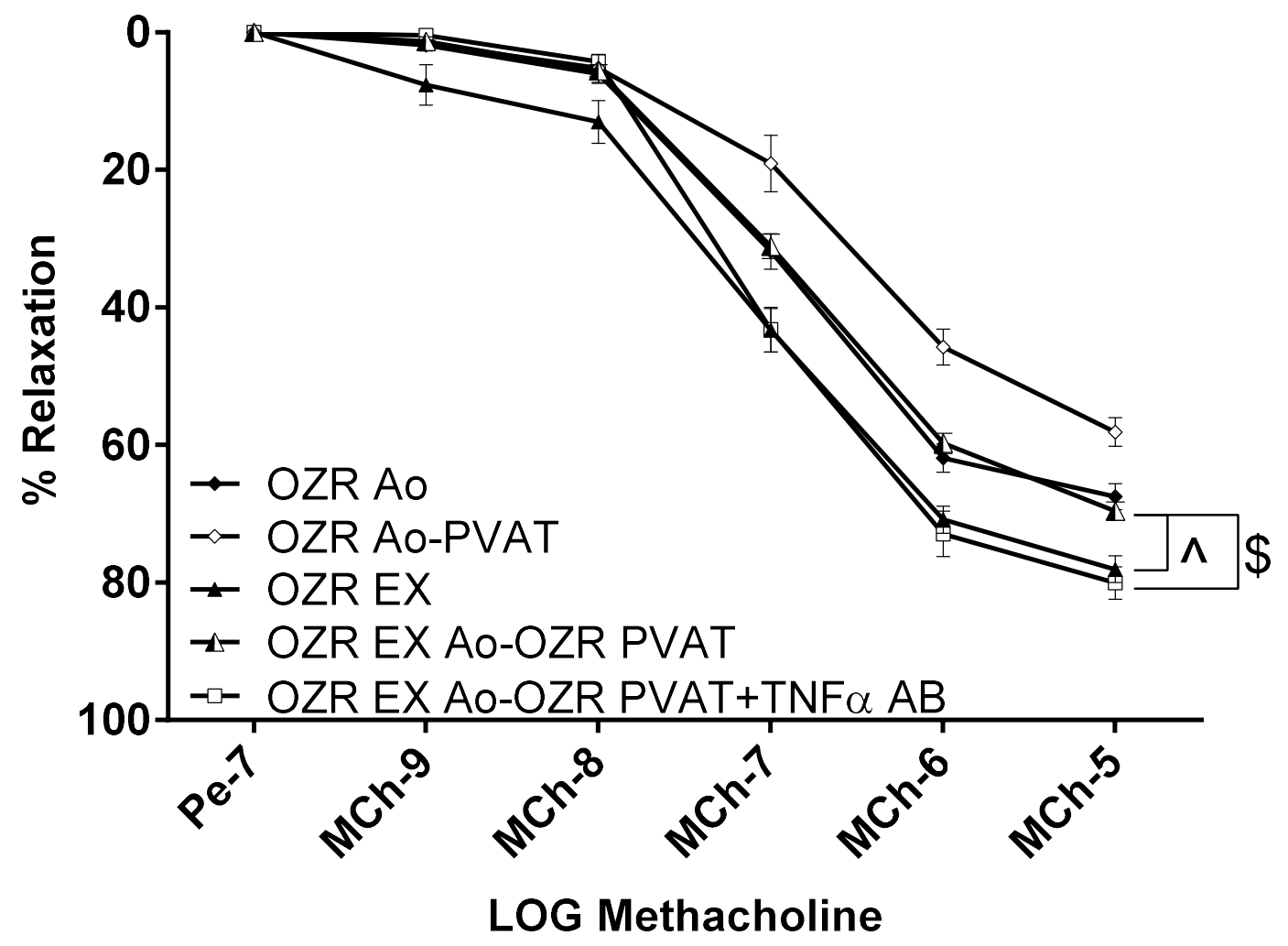


A

B

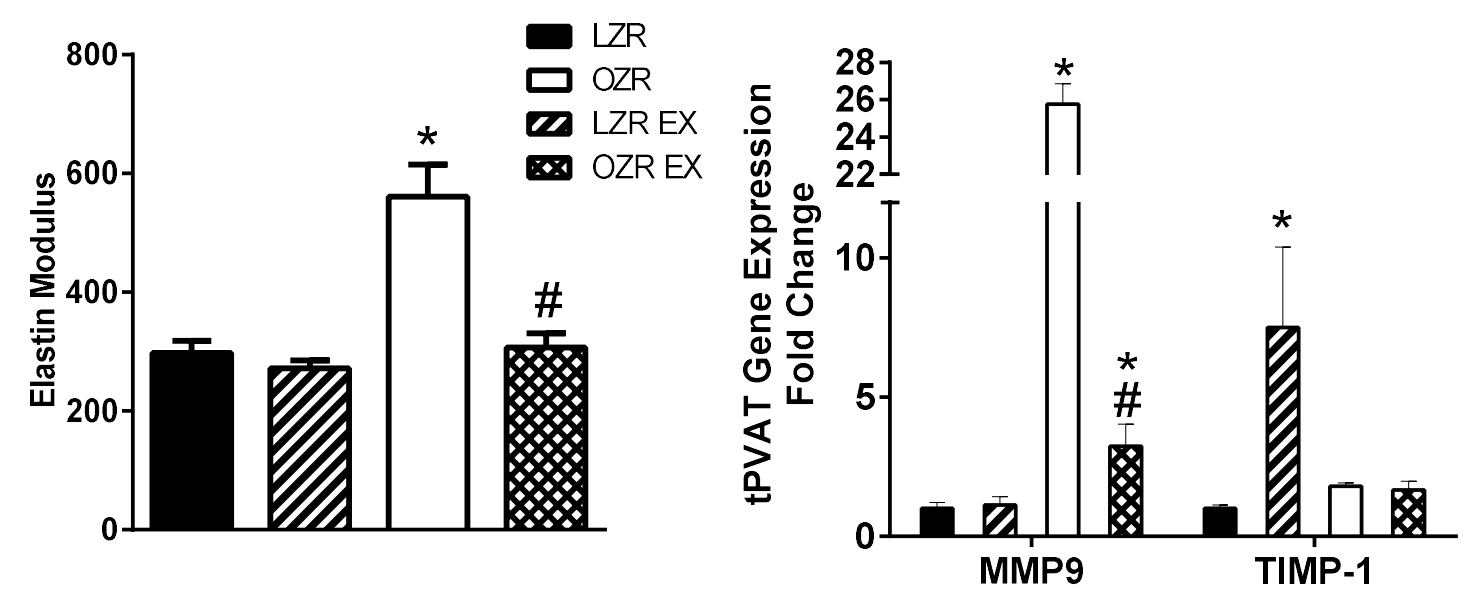

C
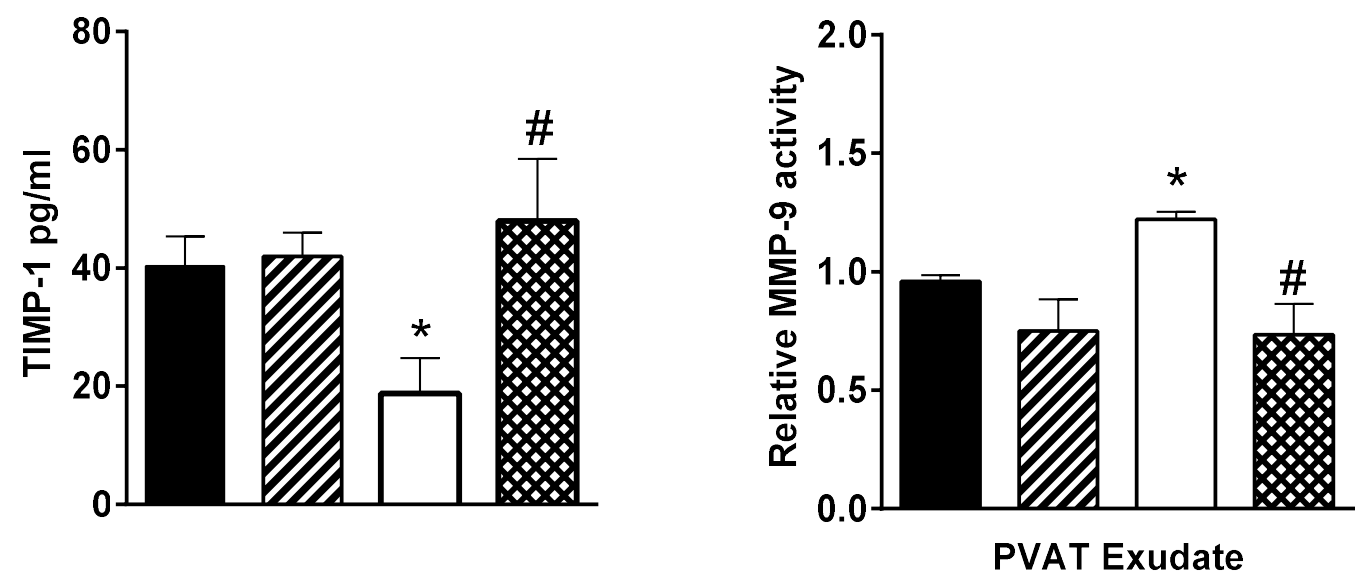


\title{
Chapter 5: Chronic Mild Stress Induced Perivascular Adipose Tissue Impairment of Aortic Function and the Therapeutic Effect of Exercise in Lean and Obese Zucker Rats.
}

\author{
Evan DeVallance ${ }^{1}$, Kayla W Branyan ${ }^{1}$, Kent Lemaster ${ }^{2}$, I. Mark Olfert ${ }^{1}$, Emidio E Pistilli ${ }^{1}$, \\ Randall Bryner ${ }^{1}$, Jefferson C Frisbee ${ }^{2,3}$, Paul D. Chantler ${ }^{1}$ \\ ${ }^{1}$ Department of Exercise Physiology, West Virginia University School of Medicine, \\ Morgantown, WV; ${ }^{2}$ Department of Physiology and Pharmacology, Schulich School of Medicine \\ and Dentistry, University of Western Ontario, London, ON, Canada; ${ }^{3}$ Department of Medical \\ Biophysics, Schulich School of Medicine and Dentistry, University of Western Ontario, London, \\ ON, Canada
}

Running title: UCMS and Exercise Effects on tPVAT

Corresponding author

Paul D Chantler, One Medical Center Drive, Morgantown, WV, 26505, pchantler@hsc.wvu.edu,

tel: $\quad$ (304) 293-0646

fax: (304) 293-7105

Keywords: Perivascular adipose tissue, metabolic syndrome, inflammation, angiotensin II, aldosterone, exercise, UCMS, aortic stiffness.

Word count: 7839

Figures: 6 Tables: 1 


\section{Abstract:}

Depressive disorders are common worldwide and in the United States, with an alarmingly high prevalence amongst MetS patients. Endeavors into the pathophysiological impacts of depressive states has uncovered many links to vascular impairment and risk of cardiovascular disease. In recent studies perivascular adipose tissue (PVAT) has been shown to regulate vascular impairment in various disease states. However, the impact of depressive state and the comorbidity of depressive states with MetS on thoracic (t)PVAT are unknown. Additionally, exercise training is known to combat the pathology of both MetS and depressive states, but the role of tPVAT in these actions are unknown. To assess these gaps in knowledge we will implement the chronic mild stress protocol (UCMS) and treadmill exercise (Ex) in lean (LZR) and MetS (OZR) rats. UCMS resulted in increased secretion of Ang II and decreased release of adiponectin in both LZR and OZR tPVAT. UCMS tPVAT subsequently reduced aortic EDD in both LZR $(\mathrm{p}<0.05)$ and OZR $(\mathrm{p}<0.05)$. UCMS caused increased stiffness in LZR through actions of tPVAT, while no effect was seen in OZR. Ex prevented the tPVAT impairment of aortic relaxation associated with UCMS in both LZR $(\mathrm{p}<0.05)$ and OZR $(\mathrm{p}<0.05)$. Additionally, Ex reduced aortic stiffness in both LZR and OZR. These beneficial regulations of the aorta are likely due to Ex prevention of increased Ang II and reduced adiponectin associated with UCMS in tPVAT. In conclusion UCMS alters tPVAT secretion profile leading to the activation of aortic ROS and impairment of function. These effects of UCMS can be prevented by Ex. 


\section{Introduction:}

Depressive psychological disorders are a common worldwide affliction affecting approximately 350 million people making it the leading global cause of disability according to the world health organization. The burden of depressive states is at its highest in the USA with prevalence rate of roughly $17 \%$ [1]. The complex psychophysiological interactions of depressive states cause numerous health issues including being an independent risk factor of cardiovascular disease (CVD) [2]. Clinical evaluations of arterial stiffness [3], a major CVD risk factor [4], and flow mediated dilation [5], link vascular impairment with depressive symptoms.

The link between depressive states and vascular function have slowly come to light in recent history; a detailed account of the advancements in the field can be found in a review by Golbidi et. al [6]. In addition, there is a high co-prevalence of depressive states with obesity and the metabolic syndrome (MetS) [7, 8], a disease state with significant pre-existing vascular dysfunction [9-12]. A growing body of work shows perivascular adipose tissue (PVAT) partially mediates the progression and development of vascular diseases. Our own previous work in a rodent model of MetS (obese Zucker Rat, OZR) indicates thoracic (t) PVAT cytokine alterations mediate aortic dysfunction through activation of aortic reactive oxygen species (ROS) production. Despite growing evidence linking depressive states with the development of vasculopathies important questions remain. 1) Does the development of depressive states affect tPVAT or tPVAT mediated aortic function and 2) is this relationship affected by the comorbidity of MetS? 
Aerobic exercise training (Ex) has been utilized as a therapy for depressive states and to yield improvements in vascular function in the MetS . Aerobic exercise (Ex) promotes vascular protection through its anti-inflammatory and anti-oxidative effects REF. However, the effect of Ex on TPVAT and its regulation of aortic function is unknown with concurrent UCMS and the comorbidity of UCMS and MetS. We have previously shown Ex in the OZR is beneficial in restoring aortic function and tPVAT mediation of aortic function (ref). Indeed, Ex in OZR resorts the tPVAT phenotype and tPVAT promotion of NO signaling in the aorta. Thus, given our previous work examining the beneficial effects of Ex on vascular function, specifically the its role on tPVAT regulation, we asked the following question: Can Ex during the development of MetS and depressive states, limit the aortic and tPVAT dysfunction?

In the study, we aim to determine the impact of UCMS on tPVAT and tPVAT regulation of aortic function with and without concomitant MetS. We hypothesize UCMS will impairer tPVAT function and upregulate pro-inflammatory cytokines causing impairment of aortic endothelial function in both lean and MetS compared to controls. We further hypothesize the implementation of Ex alongside UCMS will prevent tPVAT impairment and improve tPVAT regulation of aortic function.

\section{Methods}

Animals and Ex Intervention: Male lean (LZR, $n=24)$ and OZR $(n=24)$ were purchased from Envigo Laboratories at 7-9 weeks of age. Animals were housed at the West Virginia University Health Science Center (WVUHSC) animal care facility on an approved protocol by the WVUHSC Animal Care and USE Committee. Animals received standard chow and tap water ad 
libitum. LZR and OZR were randomly assigned into control, UCMS (LZR-UMCS, n=8 \& OZRUMCS, n=8), or combination of UCMS and Ex groups (LZR-UCMS+Ex, n=8 \& OZRUCMS+Ex, $\mathrm{n}=8)$.

UCMS Protocol: The unpredictable chronic mild stress (UCMS) protocol is a well-defined model to induce a depressive state in rodents $[13,14]$. Rodents undergoing UCMS manifest with clinically relevant depressive symptoms such as anhedonia and learned helplessness $[13,14]$ with alterations in brain structure and function parallel to clinical depression. Rats were singly housed in UCMS groups, and exposed to the following mild environmental stressors in randomly chosen sequences for 8 hours each day, 5 days/week, over the course of 8 weeks:

1. Damp bedding - $10 \mathrm{oz}$. of water was added to each standard cage

2. Bath - all bedding was removed and $\sim 0.5$ inches of water was added to empty cage. Water temperature was room temperature, $\sim 24^{\circ} \mathrm{C}$

3. Cage Tilt - cage was tilted to 45 degrees without bedding

4. Social stress - each rat was switched into a cage of a neighboring rat

5. No bedding - all bedding was removed from the cage

6. Alteration of light/dark cycles -turning lights off/on in random increments for scheduled period.

UCMS and Exercise Combination Protocol: LZR-UCMS+Ex and OZR-UCMS+Ex underwent 8 weeks of treadmill running. Animals ran 5 days/week in individual lanes on a motor driven treadmill at a $5 \%$ grade. During the first week, animals were acclimatized to the treadmill by progressively increasing running time form 20 minutes until a duration of 60 minutes was 
achieved. A maximum speed test was then performed on each animal and target running speed was set for $60-70 \%$ of that maximum. Workouts for the following 7 weeks were 60 minutes in duration and consisted of 15 minutes of gradual increases in speed until reaching target speed, which was maintained for remaining 45 minutes. Mild electrical stimulation was used to encourage running. treadmill running was performed first thing in the morning immediately followed by subjection to the UCMS protocol as described previously.

Coat Score and Adrenal Weights: This evaluation was done for every group throughout the duration of the 8-week protocol. Each week, the rats were weighed and inspected for grooming habits. The total cumulative score was computed by giving an individual score of 0 (clean) or 1 (dirty) to eight different body parts. During terminal procedures adrenal glands were removed and cleaned under a dissecting microscope. Subsequently, adrenal glands were patted dry and weighed individually and then averaged together for each animal and were normalized to body weight.

Terminal Procedures: Importantly, terminal procedures were performed a minimum of 48 hours following the last bout of Ex to eliminate the acute effects of exercise on experiments. At time of terminal procedures, animals were weighed then deeply anesthetized with pentobarbital sodium $(50 \mathrm{mg} / \mathrm{kg}$ ip). All rats then received carotid artery and jugular vein cannulation to measure mean arterial pressure and to administer heparin, respectfully. The aorta with the surround tPVAT was removed and processed as previously published.

Assessments of gene expression, tPVAT function, and aortic function were conducted as previously described in detail (ref). Hereafter experimental methods are briefly described. 
Gene Expression: To assess gene expression, tPVAT was prepared and qrt-PCR carried out using the RNeasy Lipid Tissue Mini Kit (Qiagen) and Qiagen automated pipetting machines. $20 \mu \mathrm{L}$ PCR reactions were mixed with QuantiTect primer assays and QuantiFast PCR master mix (Qiagen). Relative quantification was carried out by the $2^{\wedge}(\mathrm{ddCt})$ method.

Measurement of ROS: Dihydroethidium (DHE, Invitrogen D1168) assays were performed on unfixed aortic rings and tPVAT sections placed in individual wells of a 96 well plate containing HEPES buffer with the following treatments: control (no added drug), tPVAT, Crossover OZR tPVAT (i.e., tPVAT from OZR on OZR-EX aortic ring or 4-Hydroxy-TEMPO (TEMPOL, $100 \mu \mathrm{M}$, Sigma-Aldrich 176141). Following completion of DHE incubation rings and PVAT were washed in HEPES buffer, placed separately in Optimal Cutting Temperature compound (OCT, Fisher Healthcare ${ }^{\mathrm{TM}}$ Tissue-Plus ${ }^{\mathrm{TM}}$ O.C.T Compound) frozen and then cut and stained/mounted with DAPI mounting media (VECTORSHEILD antifade mounting media with DAPI, Vector laboratories). Slides were imaged with an EVOS fluorescent microscope (Invitrogen EVOS FL Auto Cell Imaging System), 3 sections per image-treatment, and analyzed in ImageJ as fluorescent density/nucleus, the mean of the 3 images/treatment were used as the mean for each animal. Values were normalized to signal form TEMPOL treatment to eliminate background.

NO Bioavailability: Aortic rings were placed in individual wells of a 96 well plate containing HEPES buffer and 4-Amino-5-Methylamino-2',7'- Difluorofluorescein Diacetate (DAF-FM-DA,

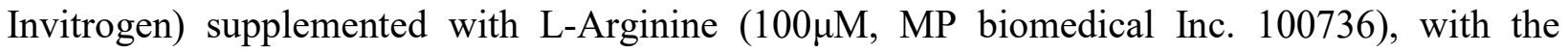


following treatments: control (no added drug), PVAT, Crossover PVAT, PVAT+TNF $\alpha$ AB $(4 \mu \mathrm{M})$ or nitro-L- arginine methyl ester (L-NAME, a potent inhibitor of NO synthase, SigmaAldrich N5751) and then stimulated with Acetyl- $\beta$-methylcholine chloride (methacholine (MCh), $1 \mathrm{X} 10^{-6}$, Sigma-Aldrich A2251). The conditioned solution was read in a plate reader excitation/emission at 495/515nm wavelength. Fluorescence was normalized to aorta length and L-NAME value.

tPVAT Cytokine Profile: tPVAT at a ratio of $200 \mathrm{mg} / 1 \mathrm{~mL}$ was incubated in HEPES buffer for 1 hours at $37^{\circ} \mathrm{C}$. The tPVAT was then removed and the media was snap frozen and stored at $80^{\circ} \mathrm{C}$. The conditioned media was then run on MSD multiplex rat inflammatory panel 2 (Mesoscale discovery, V-plex K15059D-2), MMP-9 activity ELISA (Biotrak activity assay), and High molecular weight adiponectin ELISA (Mybiosource MBS020496). Additionally, tPVAT homogenates were prepared and run on MSD inflammation panel 1 rat (Mesoscale discovery, K15179C-9), aldosterone ELISA (Mybiosource MBS731388), and angiotensin II ELISA (Spibio, A05880). All assays were run per manufacturer's instructions. Additionally, tPVAT homogenates were used to run a SOD activity assay (Sigma-Aldrich 19160-1KT-F) to manufacturer's specifications.

Aortic Reactivity: Endothelial dependent dilation (EDD) and the effect of tPVAT on EDD were assessed in aortic rings cleaned and mounted in a myobath chamber between a fixed point and a force transducer (World Precision Instruments). The equilibrated aortic rings were constricted with phenylephrine and exposed to increasing doses of $\mathrm{MCh}\left(1 \mathrm{X} 10^{-9} \mathrm{M}\right.$ to $\left.1 \mathrm{X} 10^{-5} \mathrm{M}\right)$. Additional responses were carried out with either tPVAT incubation or L-NAME as previously described. 
Aortic Stiffness: Aortic rings were incubated in $\mathrm{Ca}^{2+}$ free Van Breemen solution to elicit a passive state. The rings were then mounted on an automated motorized force transducer (Aurora Scientific Inc. model $6350 * 358$ ) and force output was recorded in lab chart software by powerlab (AD instruments). After preconditioning rings were stretched to $10 \mathrm{mN}$ of force for 3 minutes during which time the internal diameter and wall thickness were measured. Subsequently, the automated force transducer increased the aortic ring diameter by $25 \%$ of initial internal diameter every 3 minutes until mechanical failure (determined by a drop in force following a stretch). Elastin modulus was determined as the slope of the stress-strain relationship.

PVAT Culture Studies: To determine the direct impact of PVAT on mechanical stiffness, LZR aortic rings $(\mathrm{n}=4 /$ treatment $)$ were cultured for 72 -hours in RMPI + GlutaMAX $^{\mathrm{TM}}+25 \mathrm{mM}$ HEPES media (gibco ${ }^{\circledR}$ by life techonologies ${ }^{\mathrm{TM}}$ ) with streptomycin and kept in a $\mathrm{CO}_{2}$ cell incubator at $37^{\circ} \mathrm{C}$ under $5 \% \mathrm{CO}_{2}$, under the following conditions; control (just media), LZR tPVAT, OZR tPVAT, LZR-UCMS tPVAT, OZR-UCMS tPVAT, LZR-UCMS+Ex tPVAT or OZR-UCMS+Ex tPVAT. Media was discarded and replenished daily. Following the 72-hours of culture aortic rings were subjected to the protocol described above to generate an elastic modulus.

$\underline{\text { Results: }}$

\section{Characteristics of Rats}

Table 5.1 displays the characteristics of the experimental groups. The components of the MetS were largely unaffected by UCMS or the combination of UCMS+Ex. Additionally, confirmation 
of the stress induced states following UCMS were validated by increased corticosterone, a reduced grooming habit as measured by coat score, and increased adrenal weights.

Aortic reactivity

Following 8-weeks of UCMS aortic EDD was impaired $\sim 10 \%$ in LZR-UCMS compared to LZRcontrols $(\mathrm{p}<0.05$, Fig.5.1A). While no differences were noted in aortic EDD between OZRUCMS and OZR-controls (Fig.5.1B). Ex alongside UCMS had no effect on aortic EDD (in the absence of tPVAT) as both OZR-UCMS+Ex and LZR-UCMS+Ex were not significantly different from either of their respective controls or UCMS groups (Fig.5.1A\&B).

When aortic reactivity was conducted in the presence of tPVAT, EDD was further reduced by $8 \%(\mathrm{p}<0.05)$ in LZR-UCMS, an effect in opposition to LZR control whereby LZR-tPVAT increased maximal EDD by 5\% ( $<<0.01$, Fig.5.1C). Similarly, tPVAT reduced aortic EDD to a greater magnitude in OZR-UCMS compared to OZR-control (i.e., max dilation decreased 15\% vs. $10 \% \mathrm{p}<0.05$, respectfully) (Fig.5.1D). However, Ex prevented the tPVAT impairment of EDD in both LZR-UCMS+Ex and OZR-UCMS+Ex (Fig.5.1E\&F).

Aortic reactivity conducted in the presence of the NO synthase inhibitor L-NAME showed UCMS increased the reliance on NO-independent relaxation pathways in LZR-UCMS $(p<0.05$, Fig.5.2A). No effect was seen in OZR-UCMS as OZR control already presented with an increased NO-independent portion of relaxation (Fig.5.2B). Ex reduced the reliance on NOindependent mechanisms of relaxation in LZR-UCMS+Ex compared to LZR-UCMS ( $<<0.05$, Fig.5.2A) and OZR-UCMS+EX compared to OZR control and OZR-UCMS (p<0.05, Fig.5.2B). 


\section{Effect of UCMS on tPVAT}

tPVAT and Aortic ROS

Aortic ROS production was higher in the LZR-UCMS vs. LZR-control group $(\mathrm{p}<0.001$, Fig.5.2C). In addition, incubation of the LZR-UCMS aorta with its tPVAT further increased aortic ROS production (Fig.5.2C). There were minimal differences in aortic ROS production between LZR-UCMS+Ex and LZR-UCMS. However, Ex did prevent the activation of aortic ROS production when incubated with its tPVAT (Fig.5.2C). In OZR-UCMS, aortic ROS production did not differ from OZR control; in contrast, incubation with its tPVAT significantly elevated aortic ROS production (Fig.5.2D), and to a higher level than in the OZR control (Fig. 5.2D). Aortic ROS production did not differ between OZR-UCMS+Ex and OZR-UCMS, but Ex in the OZR-UCMS+Ex group did prevent the tPVAT activation of aortic ROS (Fig.5.2D).

tPVAT and Aortic NO

LZR-UCMS presented with lower NO bioavailability than LZR-controls, and aortic NO bioavailability was further reduced by $12 \%$ following tPVAT incubation (Fig.5.2E). Whereas, aortic NO bioavailability was higher $(\mathrm{p}<0.05)$ in the LZR-UCMS+Ex in the absence and presence of tPVAT compared to LZR-UCMS (Fig.5.2E). In the OZR's, no differences were noted in aortic NO bioavailability in the UCMS and control groups. However, when the OZRUCMS aorta was incubated with the OZR-UCMS tPVAT, NO bioavailability was significantly decreased ( $p<0.05$, Fig.5.2F). Ex had minimal effects in the OZR, whereby aortic NO bioavailability did not differ between OZR-UCMS+EX and OZR-UCMS. Incubation of the OZR-UCMS+Ex aorta with its TPVAT did not improve or decrease NO bioavailability, which 
yielded higher NO compared to both OZR control and OZR-UCMS aortas incubated with tPVAT (Fig.5.2F).

\section{Effect of UCMS and Exercise on tPVAT Environment}

tPVAT ROS, Anti-oxidant Defense, and Immuno-attraction

tPVAT ROS production was higher in both LZR-UCMS and OZR-UCMS compared to their respective controls (Fig.5.3A). In contrast, tPVAT ROS production was lower in LZRUCMS+Ex and OZR-UCMS+Ex vs. the their respective UCMS groups (Fig.5.3A). Additionally, SOD activity was impaired by 50\% in LZR-UCMS vs. LZR-controls, but not in OZR-UCMS vs. OZR-controls, whereas tPVAT relative SOD activity was higher in the LZR-UCMS+Ex and OZR-UCMS+Ex vs. LZR and OZR UCMS groups (Fig.5.3B). Immuno-attractants MCP-1 and lipocalin-2 were significantly 50\% higher in the LZR-UCMS compared to LZR control, but 2550\% lower in LZR-UCMS+Ex vs. LZR UCMS group (Fig.5.3C). UCMS had no effect on immune-attractant cytokines in OZR-UCMS, but in the OZR-UCMS+Ex group KC/GRO was $35 \%$ lower compared to OZR UCMS, and 60\% lower compared to OZR-controls $(\mathrm{p}<0.05$, Fig.5.3D).

tPVAT Cytokine and Hormone Release

Along with the increased tPVAT ROS production, a higher $(\mathrm{p}<0.05)$ release of pro-inflammatory cytokines from the tPVAT in the LZR UCMS was noted, specifically TNF $\alpha$ (120\%), IL-1b (100\%), IFN- $\gamma(200 \%)$, IL-6 (300\%), and TSP-1 (50\%) were higher vs. LZR-controls (Fig.5.4AE). Interestingly, only IFN- $\gamma$ and TSP-1 were lower $(p<0.05)$ in the LZR-UCMS+Ex vs. LZR- 
UCMS group (Fig.5.4C\&E). Surprisingly, pro-inflammatory cytokines were not different between OZR-UCMS and OZR-controls. However, OZR-UCMS+Ex did have a lower $(\mathrm{p}<0.05)$ IL-1 $\beta$ and IL-6 compared to OZR-UCMS, and a lower TNF $\alpha$ and IL-6 compared to OZRcontrols (Fig.5.4A).

Anti-inflammatory cytokines were reduced by UCMS, specifically IL-4 (70\%), IL-5 (60\%), IL$13(55 \%)$, and IL-10 (75\%) were lower $(\mathrm{p}<0.01)$ in the LZR-UCMS vs. LZR-controls (Fig.5.4F\&G), whereas, both IL-4 and IL-10 were higher $(\mathrm{p}<0.05)$ in the LZR-UCMs+Ex vs. LZR-UCMS group (Fig.5.4F\&G). While, the anti-inflammatory cytokines did not differ between OZR-UCMS and OZR-controls (Fig.5.4H), IL-10 was higher $(\mathrm{p}<0.05)$ in OZR-UCMS+Ex compared to OZR-controls (Fig.5.4F).

The NO promoting cytokine HMW adiponectin was $70 \%$ lower $(\mathrm{p}<0.01)$ in LZR-UCMS compared to LZR-control (Fig.5.4I), and Ex restored adiponectin levels in LZR-UCMS+Ex back to LZR-control (Fig.5.4I). A similar pattern was observed in OZRs, whereby adiponectin was $65 \%$ lower $(\mathrm{p}<0.01)$ in OZR-UCMS compared to OZR-control, but no differences were noted in adiponectin between OZR-UCMS+Ex and OZR-controls (Fig.5.4I).

Since the pro-inflammatory cytokines were unable to explain the increased ROS and decreased aortic EDD we explored stress induced pathways. Angiotensin II (Ang II) was 50\% higher $(p<0.05)$ in both LZR-UCMS and OZR-UCMS compared to their controls. Whereas, Ang II was lower in the LZR-UCMS+Ex and OZR-UCMS+Ex vs. their respective UCMS groups (Fig.5.5A). Gene expression of the angiotensin converting enzyme 2 was not different between 
LZR-UCMS and LZR-controls. However, expression was significantly higher in OZR control compared to LZR and expression was reduced back to LZR control levels in OZR-UCMS (Fig.5.5C). Additionally, aldosterone was higher $(\mathrm{p}<0.05)$ in OZR-UCMS vs. OZR-controls (Fig.5.5B), but lower ( $\mathrm{p}<0.05)$ in OZR-UCMS+Ex vs. OZR-UCMS (Fig.5.5B). No differences in aldosterone levels were noted between the LZR groups.

\section{Effect of UCMS and Exercise on Aortic Stiffness}

Aortic stiffness was higher $(\mathrm{p}<0.05)$ in LZR-UCMS vs. LZR controls, but lower $(\mathrm{p}<0.05)$ in LZR-UCMS+Ex vs. LZR-UCMS (Fig.6A). On the contrary, aortic stiffness was similar between OZR-controls and OZR-UCMS, but the aortic stiffness was lower $(\mathrm{p}<0.05)$ in OZR-UCMS+Ex compared to both OZR-UCMS and OZR-controls (Fig.5.6A).

To examine the direct role of tPVAT in aortic stiffness we co-cultured healthy LZR vessels with tPVAT from the various experimental groups. Similar to elastin modulus in the uncultured aortas, aortic stiffness was $20 \%$ higher $(\mathrm{p}<0.05)$ with the LZR-UCMS tPVAT compared to media control (Fig.5.6B), whereas tPVAT from OZR-controls and OZR-UCMS increased aortic stiffness to the same degree (Fig.5.6B). Ex prevented the tPVAT mediated increased stiffness in both LZR-UCMS+Ex and OZR-UMCS+Ex (Fig.5.6B). We also examined MMP-9 activity and TIMP-1 protein levels in the tPVAT. tPVAT MMP-9 activity was higher $(\mathrm{p}<0.05)$ in LZR-

UCMS vs. LZR-controls (Fig.5.6C), but lower $(\mathrm{p}<0.05)$ in the LZR-UCMS+Ex compared to LZR-UCMS (Fig.5.6C). Of note, MMP-9 activity was unchanged in all OZR groups (Fig.5.6C). Additionally, the inhibitor TIMP-1 which did not differ between LZR-controls and LZR-UCMS 
was lower in the LZR-UCMS+Ex vs. LZR controls. In the OZR groups we only established a trend for TIMP-1 to be higher in the OZR-UCMS+Ex vs. OZR-controls (Fig.5.6D).

\section{Discussion:}

For first time our study shows 3 important findings regarding the effect of UCMS alone and with MetS on tPVAT and tPVAT regulation of aortic function. 1) UCMS in LZR increased production of Ang II and TNFa in tPVAT accompanied by increased tPVAT ROS production; alteration in TPVAT caused activation of aortic ROS, reduced NO bioavailability, diminished EDD, and increased stiffness compared to the media control. 2) Contrary to findings in LZR, OZR-UCMS did not alter aortic NO or EDD compared to controls without the presence of tPVAT. However, in the presence of tPVAT aortic ROS was activated resulting in reduced NO and EDD in OZR-UCMS compared to OZR control, likely due to tPVAT increased Ang II and aldosterone. 3) The major effect of Ex was reduced Ang II and higher HMW adiponectin in tPVAT, which removed the UCMS associated detrimental impact of tPVAT on aortic function

\section{UCMS Impairments of tPVAT and Aortic Function}

Aortic Dysfunction in UCMS

It has been previously shown that exposure to chronic daily stress impairs aortic EDD in healthy mice, via reduced NO bioavailability that was independent of changes in eNOS expression [15].

Our data supports these findings in lean healthy rats. Further, our data suggests increased aortic ROS production may be responsible for lower NO bioavailability and the reduced aortic EDD 
observed in LZR-UCMS (without tPVAT incubation). Similar to the previous study [15], aortic relaxation after treatment with the NOS inhibitor L-NAME was higher in LZR-UCMS, likely due to the upregulation of compensatory pathways. This slight increase in NO-independent relaxation was not enough to fully compensate for the loss of NO. In opposition to our findings in healthy rats, UCMS had no effect on aortic EDD in OZR. This is likely due to the fact OZR aortas present with already impaired aortic EDD, increased ROS, and reduced NO production, which again were unaltered in OZR-UCMS. Aortic relaxation following L-NAME treatment was higher in OZR compared to LZR and OZR-UCMS was not different from OZR control. This suggest the compensatory mechanisms of aortic relaxation are induced by MetS and UCMS has no further effect. However, these compensatory mechanisms of relaxation only constitute a small portion of the relaxation response and NO still predominates.

tPVAT impairment of EDD and NO in UCMS

Previous work utilizing the UCMS model established traditional circulating markers of inflammation and oxidative stress did not correlate highly with impaired aortic function [15]. Our current work does show UCMS tPVAT actively impairs endothelial function potential through tPVAT derived hormones and inflammatory cytokines paracrine signaling. Chronic exposure to stress has been shown to increase inflammation [16-18], however, the effect on tPVAT inflammation was previously unknown.

Role of inflammatory cytokines 
These two pathways normally compete to regulate inflammatory cytokines release (SNS) or suppression (HPA axis). However, chronic exposure to stress can result in glucocorticoid resistance, which removes the major mechanism for HPA axis suppression of inflammation[19, 20]. This results in upregulation of cytokine production and release $[18,20]$. Similar to isolated immune cells $[17,18]$ and other body tissues [16], we show chronic stress increases the release of pro-inflammatory cytokines from tPVAT. The pro-inflammatory cytokines, specifically TNF $\alpha$ [21] and IL-1 $\beta$ [22], are known activators of oxidative enzymes and likely contributed to the increased activation of aortic ROS production by tPVAT in LZR-UCMS. Additionally, these cytokines may act through autocrine signaling to promote pro-inflammatory gene expression [23-25], as well as, activation of oxidative enzymes in tPVAT, perpetuating tissue dysfunction. Further experiments are needed to fully understand how tPVAT mediates inflammation; however, resident tPVAT macrophages likely play a significant role as it has been previously shown that social stress induces glucocorticoid resistance and increased inflammation in splenic macrophages [18]. Additionally, TNF $\alpha$ inhibits adiponectin, an important mediator of adipose NO signaling [26] and maintaining a healthy phenotype [27], was decreased in the UCMS groups. Taken together, UCMS induces expression of the inflammatory cytokine TNF $\alpha$ in LZRUCMS tPVAT similar to OZR controls, and likely represents a key mediator of tPVAT dysfunction. Interestingly, increased inflammation was not observed in OZR-UCMS compared to OZR controls, this is likely due to the already increased SNS activation [28] and inflammation associated with the MetS phenotype (chapter3). This might suggest that the MetS and the development of a depressive like state (via UCMS) act through similar pathways to activate proinflammatory cytokines to be released in tPVAT. Of note, not only is TNF $\alpha$ elevated as an outcome of UCMS, but TNF $\alpha$ has been shown to mediated stress-induced depression and TNF 
receptor knockout mice present with an anti-depressive phenotype [29]. This might suggest that our OZR controls may suffer some level of depressive symptoms.

\section{UCMS effect of tPVAT Angiotensin II and Aldosterone}

In addition to the effect on inflammation, chronic stress activates the renin-angiotensinaldosterone-system (RAAS) [30]. The presence of RAAS in adipose tissue and its role in CVD has been well defined [31]. We therefore assessed secretion of the key vasoactive hormones Ang II and aldosterone from tPVAT. We showed Ang II secretion from tPVAT was elevated in both LZR-UCMS and OZR-UCMS compared to their respective controls. Further, Ang II was elevated in the OZR control vs. LZR control, so unlike inflammatory cytokines, UCMS results in higher levels of Ang II released from tPVAT above the already elevated levels in OZR control. The increased Ang II with UCMS may be due to the decreased ACE 2 gene expression with UCMS. ACE 2 metabolizes Ang II to Ang 1-7 [32] and is an important PVAT derived vasodilator [33]. Autocrine signaling of Ang II may alter adipose blood flow distribution, increase SNS activity, increase oxidative stress and promote lipogenesis [34, 35]. Suggesting the actions of Ang II in LZR-UCMS tPVAT might be the cause for the increasing oxidative load and additionally the increased TNF $\alpha$ secretion [36, 37]. Upregulation of Ang II may also account for the decreased HMW adiponectin following UCMS in tPVAT as blockade of Ang II receptors was shown to increase adiponectin gene expression [38]. In OZR-UCMS Ang II did not augment pro-inflammatory cytokines likely due to the already elevated levels of ROS and inflammation. However, unlike in LZR-UCMS, Ang II stimulated the increase of aldosterone in OZR-UCMS. Ang II stimulation pathway of adipose aldosterone was recently uncovered and has been 
implicated in obesity related vascular impairment [39]. Aldosterone's mechanism of vascular impairment is likely a result of oxidative enzymes activation [40].

Ang II is the common vaso-active link between LZR-UCMS and OZR-UCMS. Increased release of Ang II following UCMS may impacts aortic reactivity in 3 ways. First Ang II is a potent stimulator of vascular ROS through activation of NOX [41] subsequently reducing NO. Secondly, Ang II enhances the vascular signaling of the vasoconstrictor endothelin-1 [42]. Finally, Ang II stimulates vascular inflammation [36]. Additionally, UCMS caused reduction of HMW adiponectin, potentially through Ang II or TNF $\alpha$. HMW adiponectin promotes aortic NO production through rapid non-genomic and long term genomic pathways [26], suggesting the reduced levels observed in the current study may contribute to the diminished NO bioavailability and EDD following UCMS. In LZR-UCMS elevated Ang II was accompanied by elevated inflammatory cytokine TNF $\alpha$, IFN- $\gamma$, and TSP-1. TNF $\alpha$, IFN- $\gamma$, and TSP-1 have all been shown to impair NO and EDD [43-45] . Additionally, TNF $\alpha$ can stimulate aortic ROS by NOX and inhibit adiponectin interfering with NO bioavailability [26, 46, 47]. In contrast, in OZR-UCMS, Ang II caused the increased release of aldosterone from tPVAT. Similar to AngII and the cytokines discussed above aldosterone impairs EDD through increasing ROS production [48]. Additionally, aldosterone is proposed to increase endothelin sensitivity and cause endothelial swelling and rigidity [49], which may reduce NO [50]. More in-depth studies are needed to detail the direct adipose signaling pathways responsible for Ang II upregulation and downstream signaling in stress/depression and in combination with MetS.

UCMS effect on aortic stiffness 
Increased aortic stiffness is a classic cardiovascular risk factor, with significant clinical relevance, and is an independent predictor of cardiovascular morbidity and mortality [51, 52]. Are data showed increased aortic stiffness in the LZR-UCMS, however, no effect was noted in the OZR-UMCS vs. OZR controls. Like the lack of change in EDD in the OZR-UMCS, the lack of effect seen in aortic stiffness in the OZR-UCMS is likely due to the already elevated aortic stiffness in OZR. Our aorta-tPVAT culture studies provided some insight to the role of tPVAT on aortic stiffness. LZR-UCMS tPVAT increased aortic stiffness of a LZR control aorta significantly compared to media control, but only trended for significance compared to LZR control tPVAT. Though this effect did not reach significance we believe it still highlights a detrimental impact of UCMS tPVAT on aortic stiffness. This is supported by increased MMP9 activity in LZR-UCMS tPVAT exudate. This increased MMP9 activity may, in part, be due to the increased TNF $\alpha$ levels, which mediates activation of MMP9 [53]. A similar effect is evident in OZR control tPVAT, which we have previously shown to be dependent on TNF $\alpha$ (chapter3). The culture study supports the idea of tPVAT mediated stiffening in the aorta as it increased stiffness compared to the media control. However, it did not reach statistical difference from LZR control tPVAT we speculate this means circulating and tPVAT factor play a supportive role in increasing aortic stiffness in LZR-UCMS.

\section{Effect of Exercise in Preventing UCMS Impairment of tPVAT}

Due to the pro-inflammatory pathophysiology of both stress and depressive states and MetS, makes Ex a logical treatment option for both stress induced depressive states and MetS. From the current study, it was clear that Ex had little to no impact on UCMS induced behavioral changes 
or adrenal mass, suggesting the benefits of Ex were independent from the response to the stressful stimuli measured. However, we cannot rule out the effect of Ex on the SNS stress response as it was not measured [54].

\section{Ex Effect on Aortic ROS and Reactivity}

Ex had no effect on OZR aortic relaxation in the absence of tPVAT. While in LZR-UCMS+Ex maximal relaxation was improved compared to LZR-UCMS, the whole relaxation response did not differ from LZR UCMS or control. Ex did however, increase the NO contribution to EDD as relaxation after L-NAME treatment was the same as LZR control for both LZR and OZR UCMS+EX. This was supported by trends for increased NO in LZR and OZR UCMS+Ex aorta and decreased ROS in LZR-UCMS+Ex. While Ex had only marginal effects on aortic function in the absence of tPVAT, significant benefical effects were observed in IPVAT regulated aortic function.

\section{Effect of Exercise on tPVAT Cytokines}

Ex is known to have anti-inflammatory effects and reduce oxidative stress in the vasculature and other tissues [55]. However, to what extent Ex effects the tPVAT dysfunction after UCMS was previously unknown. There are many purposed mechanisms of exercise's anti-inflammatory effects including reduced immune infiltration into adipose tissue, repolarization of tissue resident immune cells, and endocrine signaling from Ex induced release of anti-inflammatory cytokines. The exact mechanisms of Ex signaling are beyond the scope of the study but are discussed elsewhere [56]. In the current study, Ex in combination with UCMS in LZR showed mixed success in reducing the pro-inflammatory cytokines with significant reductions only evident for 
TSP-1 and IFN- $\gamma$. In OZR-UCMS+Ex TNF $\alpha$, IL-6, and IL-1 $\beta$ were significantly reduced from either OZR control or OZR UCMS. In contrast, the anti-inflammatory cytokines, IL-10 and adiponectin were increased in both LZR-UCMS+Ex and OZR-UCMS+Ex. It has been previously shown that IL-10 and adiponectin can affect TNF $\alpha$ [57, 58], thus the higher levels of IL-10 and adiponectin in the LZR-UCMS+Ex may exert some inhibition of TNF $\alpha$ actions, (i.e. activation of ROS production) despite the fact we found no significant change in TNF $\alpha$ in LZRUCMS+Ex. These data, might suggest Ex shifts the balance away from oxidative enzyme activation to the promotion of NO, supported by the increased NO and reduced ROS in tPVAT incubated aortas in the combination groups $[26,57,58]$.

\section{Effect of Exercise on Angiotensin II}

Our data showed Ang II had uniform increases in both OZR-UCMS and LZR-UCMS and might represent a key mechanism in linking UCMS to tPVAT ROS, inflammation, and impairment of aortic function. Importantly, Ex prevented the higher levels of Ang II noted in the UCMS alone groups. The lower levels of Ang II in the UCMS+Ex groups likely represents a key mechanism for the prevention aortic EDD dysfunction in the presence of tPVAT, as it was the only marker to return to control levels, concurrently the tPVAT impairment on EDD was removed. In OZRUCMS+Ex we suggest Ex had a multifaceted effect which prevented the impairment of tPVAT due to both MetS and UCMS. The major effect of Ex was in the prevention of aortic ROS activation via reduced Ang II, aldosterone, and TNF $\alpha$ production.

Exercise Effect on Aortic Stiffness 
Both Ex groups had reduced aortic stiffness compared to their respective UCMS groups. Reduced aortic stiffness may, in part, be due to alterations in tPVAT signaling. Co-culture experiments showed tPVAT from both Ex groups had lower measures of aortic stiffness compared to their respective UCMS groups. The improved aortic stiffness in the LZR UCMS+Ex group likely reflects the actions of Ex on decreasing aortic and tPVAT production of ROS, and a reduction in tPVAT derived MMP-9 activity. In contrast, in the OZR-UCMS+Ex group, MMP-9 activity and TIMP1 levels in tPVAT were not different compared to the OZRUCMS group. However, tPVAT activation of aortic ROS production was reduced in the OZRUCMS+Ex, suggesting that this is likely the main pathway by which Ex improved aortic stiffness in the OZR.

\section{$\underline{\text { Limitations }}$}

A limitation of the study was that Ex was introduced alongside UCMS, and results should be interpreted as such. However, quality control measures of UCMS, coat score and adrenal weight showed the combination groups were adequately stressed under the protocol. Additional investigations into the efficacy of Ex in reversing the effects of established UCMS are warranted. Another limitation is the lack of intraluminal flow in the co-culture experiments, which is an important for shear stress mediated release of $\mathrm{NO}$ and regulation of stiffness. However, the use of a media control helps to account for the increase in stiffness due to the lack of flow.

\section{Conclusion:}

In conclusion, this study shows UCMS impairs tPVAT, regulating activation of aortic ROS and decreasing NO regardless of MetS status. UCMS had both common and divergent (dependent on 
MetS status) effects on tPVAT. Two common features of UCMS impact on tPVAT were increased Ang II and reduced HMW adiponectin, which likely contributes to the increased activation of aortic ROS by UCMS tPVAT and reduced NO bioavailability in aortas incubated with UCMS tPVAT. Uniquely in LZR, UCMS elevated pro-inflammatory cytokines (i.e. TNF $\alpha$, TSP-1, and IL-1 $\beta$ ), which likely contributes to aortic impairment of EDD and stiffness through actions on MMP9. Alternatively, in OZR, UCMS elevated tPVAT aldosterone, which may contribute to the production of ROS. Implementation of Ex alongside UCMS, prevented the increased Ang II and reduced HMW adiponectin in tPVAT associated with UCMS. Additionally, Ex reduced aldosterone and pro-inflammatory cytokines in OZR-UCMS+Ex. The restoration of tPVAT secretion profile prevented the tPVAT impairment of aortic EDD and stiffness, potentially through reduced activation of aortic ROS.

\section{$\underline{\text { References: }}$}

1. National Survey on Drug Use and Health (U.S.) and United States. Substance Abuse and Mental Health Services Administration. Office of Applied Studies., Results from the ... National Survey on Drug Use and Health. National findings, in National Survey on Drug Use and Health series. 2015, Dept. of Health and Human Services, Substance Abuse and Mental Health Services Administration, Office of Applied Studies: Rockville, MD. p. 7 volumes.

2. Van der Kooy, K., et al., Depression and the risk for cardiovascular diseases: systematic review and meta analysis. Int J Geriatr Psychiatry, 2007. 22(7): p. 613-26. 
3. Seldenrijk, A., et al., Depression, anxiety, and arterial stiffness. Biol Psychiatry, 2011. 69(8): p. 795-803.

4. Mattace-Raso, F.U., et al., Arterial stiffness and risk of coronary heart disease and stroke: the Rotterdam Study. Circulation, 2006. 113(5): p. 657-63.

5. Rajagopalan, S., et al., Abnormal brachial artery flow-mediated vasodilation in young adults with major depression. Am J Cardiol, 2001. 88(2): p. 196-8, A7.

6. Golbidi, S., J.C. Frisbee, and I. Laher, Chronic stress impacts the cardiovascular system: animal models and clinical outcomes. Am J Physiol Heart Circ Physiol, 2015. 308(12): p. H1476-98.

7. Carey, M., et al., Prevalence of comorbid depression and obesity in general practice: a cross-sectional survey. Br J Gen Pract, 2014. 64(620): p. e122-7.

8. Pratt, L.A. and D.J. Brody, Depression and obesity in the U.S. adult household population, 2005-2010. NCHS Data Brief, 2014(167): p. 1-8.

9. Brooks, S.D., et al., Metabolic syndrome impairs reactivity and wall mechanics of cerebral resistance arteries in obese Zucker rats. Am J Physiol Heart Circ Physiol, 2015. 309(11): p. H1846-59.

10. Donley, D.A., et al., Aerobic exercise training reduces arterial stiffness in metabolic syndrome. J Appl Physiol (1985), 2014. 116(11): p. 1396-404.

11. Fournier, S.B., et al., Improved arterial-ventricular coupling in metabolic syndrome after exercise training: a pilot study. Med Sci Sports Exerc, 2015. 47(1): p. 2-11.

12. Frisbee, J.C., et al., Distinct temporal phases of microvascular rarefaction in skeletal muscle of obese Zucker rats. Am J Physiol Heart Circ Physiol, 2014. 307(12): p. H171428. 
13. Mineur, Y.S., C. Belzung, and W.E. Crusio, Effects of unpredictable chronic mild stress on anxiety and depression-like behavior in mice. Behav Brain Res, 2006. 175(1): p. 43 50.

14. Willner, P., Validity, reliability and utility of the chronic mild stress model of depression: a 10-year review and evaluation. Psychopharmacology (Berl), 1997. 134(4): p. 319-29.

15. d'Audiffret, A.C., et al., Depressive behavior and vascular dysfunction: a link between clinical depression and vascular disease? J Appl Physiol (1985), 2010. 108(5): p. 104151.

16. Karagiannides, I., et al., Chronic unpredictable stress regulates visceral adipocytemediated glucose metabolism and inflammatory circuits in male rats. Physiol Rep, 2014. 2(5): p. e00284.

17. Spengler, R.N., et al., Stimulation of alpha-adrenergic receptor augments the production of macrophage-derived tumor necrosis factor. J Immunol, 1990. 145(5): p. 1430-4.

18. Stark, J.L., et al., Social stress induces glucocorticoid resistance in macrophages. Am J Physiol Regul Integr Comp Physiol, 2001. 280(6): p. R1799-805.

19. Avitsur, R., J.L. Stark, and J.F. Sheridan, Social stress induces glucocorticoid resistance in subordinate animals. Horm Behav, 2001. 39(4): p. 247-57.

20. Miller, G.E., et al., A functional genomic fingerprint of chronic stress in humans: blunted glucocorticoid and increased NF-kappaB signaling. Biol Psychiatry, 2008. 64(4): p. 26672.

21. Yang, B. and V. Rizzo, TNF-alpha potentiates protein-tyrosine nitration through activation of NADPH oxidase and eNOS localized in membrane rafts and caveolae of 
bovine aortic endothelial cells. Am J Physiol Heart Circ Physiol, 2007. 292(2): p. H95462.

22. Gurjar, M.V., et al., Role of reactive oxygen species in IL-1 beta-stimulated sustained ERK activation and MMP-9 induction. Am J Physiol Heart Circ Physiol, 2001. 281(6): p. H2568-74.

23. Turner, N.A., et al., Mechanism of TNFalpha-induced IL-1alpha, IL-1beta and IL-6 expression in human cardiac fibroblasts: effects of statins and thiazolidinediones. Cardiovasc Res, 2007. 76(1): p. 81-90.

24. Abu-Amer, Y., et al., Tumor necrosis factor-alpha activation of nuclear transcription factor-kappaB in marrow macrophages is mediated by c-Src tyrosine phosphorylation of Ikappa Balpha. J Biol Chem, 1998. 273(45): p. 29417-23.

25. Stylianou, E., et al., Interleukin 1 induces NF-kappa B through its type I but not its type II receptor in lymphocytes. J Biol Chem, 1992. 267(22): p. 15836-41.

26. Wang, Z.V. and P.E. Scherer, Adiponectin, cardiovascular function, and hypertension. Hypertension, 2008. 51(1): p. 8-14.

27. Bostrom, P., et al., A PGCl-alpha-dependent myokine that drives brown-fat-like development of white fat and thermogenesis. Nature, 2012. 481(7382): p. 463-8.

28. Carlson, S.H., et al., Elevated sympathetic activity contributes to hypertension and salt sensitivity in diabetic obese Zucker rats. Hypertension, 2000. 35(1 Pt 2): p. 403-8.

29. Liu, Y.N., et al., TNFalpha mediates stress-induced depression by upregulating indoleamine 2,3-dioxygenase in a mouse model of unpredictable chronic mild stress. Eur Cytokine Netw, 2015. 26(1): p. 15-25. 
30. Groeschel, M. and B. Braam, Connecting chronic and recurrent stress to vascular dysfunction: no relaxed role for the renin-angiotensin system. Am J Physiol Renal Physiol, 2011. 300(1): p. F1-10.

31. Thatcher, S., et al., The adipose renin-angiotensin system: role in cardiovascular disease. Mol Cell Endocrinol, 2009. 302(2): p. 111-7.

32. Chappell, M.C., et al., Novel aspects of the renal renin-angiotensin system: angiotensin(1-7), ACE2 and blood pressure regulation. Contrib Nephrol, 2004. 143: p. 77-89.

33. Lee, R.M., et al., Endothelium-dependent relaxation factor released by perivascular adipose tissue. J Hypertens, 2009. 27(4): p. 782-90.

34. Engeli, S., et al., The adipose-tissue renin-angiotensin-aldosterone system: role in the metabolic syndrome? Int J Biochem Cell Biol, 2003. 35(6): p. 807-25.

35. Kershaw, E.E. and J.S. Flier, Adipose tissue as an endocrine organ. J Clin Endocrinol Metab, 2004. 89(6): p. 2548-56.

36. Kalra, D., N. Sivasubramanian, and D.L. Mann, Angiotensin II induces tumor necrosis factor biosynthesis in the adult mammalian heart through a protein kinase C-dependent pathway. Circulation, 2002. 105(18): p. 2198-205.

37. Wolf, G., et al., Angiotensin II activates nuclear transcription factor-kappaB through AT1 and AT2 receptors. Kidney Int, 2002. 61(6): p. 1986-95.

38. Takemori, K., T. Inoue, and H. Ito, Effects of angiotensin II type 1 receptor blocker and adiponectin on adipocyte dysfunction in stroke-prone spontaneously hypertensive rats. Lipids Health Dis, 2013. 12: p. 108. 
39. Briones, A.M., et al., Adipocytes produce aldosterone through calcineurin-dependent signaling pathways: implications in diabetes mellitus-associated obesity and vascular dysfunction. Hypertension, 2012. 59(5): p. 1069-78.

40. Virdis, A., et al., Spironolactone improves angiotensin-induced vascular changes and oxidative stress. Hypertension, 2002. 40(4): p. 504-10.

41. Griendling, K.K., et al., Angiotensin II stimulates NADH and NADPH oxidase activity in cultured vascular smooth muscle cells. Circ Res, 1994. 74(6): p. 1141-8.

42. Lin, Y.J., et al., Angiotensin II enhances endothelin-1-induced vasoconstriction through upregulating endothelin type A receptor. Biochem Biophys Res Commun, 2014. 451(2): p. 263-9.

43. Javanmard, S.H. and N. Dana, The effect of interferon gamma on endothelial cell nitric oxide production and apoptosis. Adv Biomed Res, 2012. 1: p. 69.

44. Isenberg, J.S., et al., Regulation of nitric oxide signalling by thrombospondin 1: implications for anti-angiogenic therapies. Nat Rev Cancer, 2009. 9(3): p. 182-94.

45. Mandler, W.K., et al., Thrombospondin-1 mediates multi-walled carbon nanotube induced impairment of arteriolar dilation. Nanotoxicology, 2017. 11(1): p. 112-122.

46. Kim, Y.S., et al., TNF-induced activation of the Nox1 NADPH oxidase and its role in the induction of necrotic cell death. Mol Cell, 2007. 26(5): p. 675-87.

47. Li, J.M., et al., Acute tumor necrosis factor alpha signaling via NADPH oxidase in microvascular endothelial cells: role of p47phox phosphorylation and binding to TRAF4. Mol Cell Biol, 2005. 25(6): p. 2320-30.

48. $\mathrm{Pu}, \mathrm{Q}$., et al., Endothelin antagonism on aldosterone-induced oxidative stress and vascular remodeling. Hypertension, 2003. 42(1): p. 49-55. 
49. Oberleithner, H., Aldosterone makes human endothelium stiff and vulnerable. Kidney Int, 2005. 67(5): p. 1680-2.

50. Oberleithner, H., et al., Plasma sodium stiffens vascular endothelium and reduces nitric oxide release. Proc Natl Acad Sci U S A, 2007. 104(41): p. 16281-6.

51. Laurent, S., et al., Aortic stiffness is an independent predictor of all-cause and cardiovascular mortality in hypertensive patients. Hypertension, 2001. 37(5): p. 1236-41.

52. Laurent, S., et al., Aortic stiffness is an independent predictor of fatal stroke in essential hypertension. Stroke, 2003. 34(5): p. 1203-6.

53. Wu, H.T., et al., Identifying the regulative role of $\mathrm{NF-kappaB}$ binding sites within promoter region of human matrix metalloproteinase 9 (mmp-9) by TNF-alpha induction. Appl Biochem Biotechnol, 2013. 169(2): p. 438-49.

54. Mueller, P.J., Exercise training and sympathetic nervous system activity: evidence for physical activity dependent neural plasticity. Clin Exp Pharmacol Physiol, 2007. 34(4): p. $377-84$.

55. Golbidi, S., M. Badran, and I. Laher, Antioxidant and anti-inflammatory effects of exercise in diabetic patients. Exp Diabetes Res, 2012. 2012: p. 941868.

56. Gleeson, M., et al., The anti-inflammatory effects of exercise: mechanisms and implications for the prevention and treatment of disease. Nat Rev Immunol, 2011. 11(9): p. $607-15$.

57. Zemse, S.M., et al., Interleukin-10 inhibits the in vivo and in vitro adverse effects of TNFalpha on the endothelium of murine aorta. Am J Physiol Heart Circ Physiol, 2010. 299(4): p. H1160-7. 
58. Masaki, T., et al., Adiponectin protects LPS-induced liver injury through modulation of TNF-alpha in KK-Ay obese mice. Hepatology, 2004. 40(1): p. 177-84.

\section{Figure Legends:}

Figure 5.1. Effect of UCMS and Exercise on Aortic Relaxation. A\&B) The effect of UCMS and exercise on aortic reactivity in LZR and OZR and the C\&D) impact of tPVAT on UCMS on aortic reactivity $(\mathrm{n}=8)$ and $\mathrm{E} \& \mathrm{~F})$ The effect of exercise on preventing UCMS impairment of aortic reactivity by tPVAT $(\mathrm{n}=8)$. Data represented as Mean \pm SEM * denotes statistical difference compared to LZR control, \# denotes statistical difference compared to OZR control, + denotes statistical difference of UCMS from respective control group, and ${ }^{\wedge}$ denotes statistical difference, analyzed by repeated measures ANOVA with Tukey post-Hoc, $\mathrm{p}<0.05$. Pe, phenylephrine; MCh, methacholine

Figure 5.2. Effect of UCMS and Exercise on Nitric Oxide and Aortic Reactive Oxygen Species. A\&B) Aortic reactivity following the incubation of the aorta with the nitric oxide synthase inhibitor L-NAME in both LZR and OZR groups (n=4-6). C\&D) DHE measured ROS production in aortas and aortas incubated with tPVAT and the E\&F) DAF-FM measured NO production in aortas and aortas incubated with $\operatorname{tPVAT}(\mathrm{n}=8)$. Data represented as Mean \pm SEM * denotes statistical difference compared to LZR control, \# denotes statistical difference compared to OZR control, + denotes statistical difference of UCMS from respective control group, and ^ denotes statistical difference, analyzed by repeated measures ANOVA with Tukey post-Hoc, $\mathrm{p}<0.05$. Pe, phenylephrine; MCh, methacholine; DHE, dihydroethidium. 
Figure 5.3. Effect of UCMS and Exercise on tPVAT Reactive Oxygen Species and tPVAT Immuno-Attractant Release. A\&B) The DHE measured tPVAT ROS production $(\mathrm{n}=8)$ and relative SOD activity $(n=5)$. The measured levels of immune-attractants in C) LZR and D) OZR tPVAT exudate $(\mathrm{n}=5)$. Data represented as Mean \pm SEM * denotes statistical difference compared to LZR control, \# denotes statistical difference compared to OZR control, and + denotes statistical difference of UCMS from respective control group, analyzed by repeated measures ANOVA with Tukey post-Hoc, $\mathrm{p}<0.05$. $\mathrm{KC} / \mathrm{GRO}$, chemokine (C-C motif) ligand 1; MCP-1, monocyte chemoattractant protein-1

Figure 5.4. Effect of UCMS and Exercise on tPVAT Cytokines. The effect of UCMS and Exercise on A) TNF $\alpha$, B) IL-1 $\beta$, C) IFN- $\gamma$, D) IL-6, E) TSP-1, F) IL-10, G\&H) IL-4, IL-5, IL13, and I) HMW adiponectin ( $\mathrm{n}=5)$. Data is represented as Mean \pm SEM $*$ denotes statistical difference compared to LZR control, \# denotes statistical difference compared to OZR control measured by ANOVA with Tukey Post-Hoc, $\mathrm{p}<0.05$. IL, interleukin; HMW adiponectin, high molecular weight adiponectin; $\mathrm{TNF} \alpha$, tumor necrosis factor alpha; IFN- $\gamma$, interferon gamma; TSP-1, thrombospondin 1. Data is represented as Mean \pm SEM * denotes statistical difference compared to LZR control, \# denotes statistical difference compared to OZR control measured, and + denotes statistical difference of UCMS+EX compared to respective UCMS group analyzed by repeated measures ANOVA with Tukey Post-Hoc, $\mathrm{p}<0.05$. TNF $\alpha$, tumor necrosis factor alpha; IL, interleukin; IFN- $\gamma$, interferon gamma; TSP-1, thrombospondin 1; HMW adiponectin, high molecular weight adiponectin. 
Figure 5.5. Effect of UCMS and Exercise on tPVAT Angiotensin II and Aldosterone. A\&B) The effect of UCMS and Exercise on tPVAT levels of Angiotensin and Aldosterone $(\mathrm{n}=5)$ and C) gene expression of ACE2 in control and UCMS groups $(n=3)$. Data is represented as Mean \pm SEM * denotes statistical difference compared to LZR control, \# denotes statistical difference compared to OZR control measured, and + denotes statistical difference of UCMS+EX compared to respective UCMS group analyzed by repeated measures ANOVA with Tukey PostHoc, $\mathrm{p}<0.05$. ACE, angiotensin converting enzyme.

Figure 5.6. Effect of UCMS and Exercise Prevents Aortic Stiffness. A) Aortic stiffness measured by elastin modulus in aortic rings $(n=8)$ and B) LZR rings co-cultured with tPVAT from the experimental groups $(n=3-4)$. Remodeling factor expression C) MMP-9 activity in tPVAT exudate $(n=5)$, and D) tPVAT TIMP-1 protein levels $(n=5)$. Data is represented as Mean \pm SEM * denotes statistical difference compared to LZR control, \# denotes statistical difference compared to OZR control, + denotes statistical difference of UCMS+EX compared to respective UCMS group, and $\wedge$ denotes statistical difference compared to media control measured by repeated measures ANOVA with Tukey Post-Hoc, $p<0.05$. MMP, matrix metalloproteinase; TIMP, tissue inhibitor of metalloproteinase. 
Table 5.1. Assessment of Body Weight, Blood Pressure, Blood Profile, and Stress Indices

\begin{tabular}{|c|c|c|c|c|c|c|}
\hline & \multicolumn{3}{|c|}{ LZR } & \multicolumn{3}{|c|}{ OZR } \\
\hline & Control & UCMS & UCMS+EX & Control & UCMS & UCMS+EX \\
\hline Body Mass (g) & $400 \pm 8$ & $361 \pm 5^{*}$ & $343 \pm 5^{*}$ & $585 \pm 8^{*}$ & $599 \pm 11$ & $567 \pm 10$ \\
\hline MAP (mmHg) & $112 \pm 2$ & $122 \pm 3$ & $120 \pm 3$ & $139 \pm 4 *$ & $142 \pm 4$ & $132 \pm 3$ \\
\hline $\begin{array}{l}\text { Glucose } \\
\text { (mg/dl) }\end{array}$ & $98 \pm 6$ & $124 \pm 7$ & $115 \pm 8$ & $184 \pm 12^{*}$ & $230 \pm 14$ & $219 \pm 15$ \\
\hline $\mathrm{TG}(\mathrm{mg} / \mathrm{dl})$ & $25 \pm 3$ & $54 \pm 16$ & $36 \pm 3$ & $124 \pm 8^{*}$ & $114 \pm 14$ & $103 \pm 16$ \\
\hline $\begin{array}{l}\text { Corticosterone } \\
\text { (ng/ml) }\end{array}$ & $7.0 \pm 0.2$ & $8.8 \pm 0.5^{*}$ & $10.7 \pm 1.5$ & $13.8 \pm 0.6 *$ & $17.3 \pm 1.4 \#$ & $15.8 \pm 1.5$ \\
\hline $\begin{array}{l}\text { Coat Score } \\
\text { (AU) }\end{array}$ & $0.8 \pm 0.2$ & $1.8 \pm 0.2^{*}$ & $2.0 \pm 0.3^{*}$ & $2.7 \pm 0.2^{*}$ & $5.1 \pm 0.2 \#$ & $4.8 \pm 0.2 \#$ \\
\hline $\begin{array}{l}\text { Adrenal } \\
\text { weight (mg) }\end{array}$ & $16.8 \pm 0.8$ & $25.2 \pm 1.5^{*}$ & $21.4 \pm 1.1^{*}$ & $27.2 \pm 1.4^{*}$ & $32.7 \pm 2.3 \#$ & $32.0 \pm 2.4 \#$ \\
\hline $\begin{array}{l}\text { Normalized } \\
\text { adrenal } \\
\text { weight (mg/g } \\
\text { body mass) }\end{array}$ & $\begin{array}{l}0.040 \pm \\
0.001\end{array}$ & $\begin{array}{l}0.068 \pm \\
0.003^{*}\end{array}$ & $\begin{array}{l}0.063 \pm \\
0.002^{*}\end{array}$ & $\begin{array}{l}0.045 \pm \\
0.002\end{array}$ & $\begin{array}{l}0.056 \pm \\
0.003 \#\end{array}$ & $\begin{array}{l}0.061 \pm \\
0.003 \#\end{array}$ \\
\hline
\end{tabular}

Data represented as Mean \pm SEM, ${ }^{*}$ denotes statistical difference compared to LZR control and \# denotes statistical difference from OZR control measured by ANOVA with Tukey Post-Hoc $\mathrm{p}<0.05(\mathrm{n}=6-8)$. MAP, mean arterial pressure; TG, triglyceride 
Figures

Figure 5.1. Effect of UCMS and Exercise on Aortic Relaxation

A

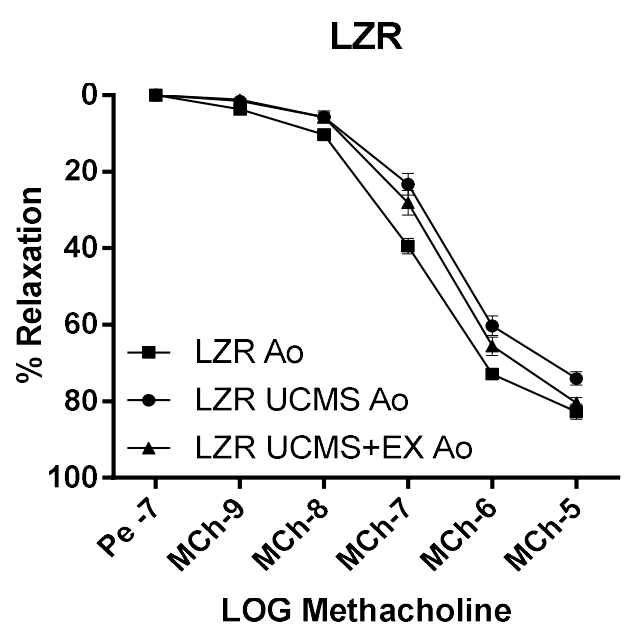

D

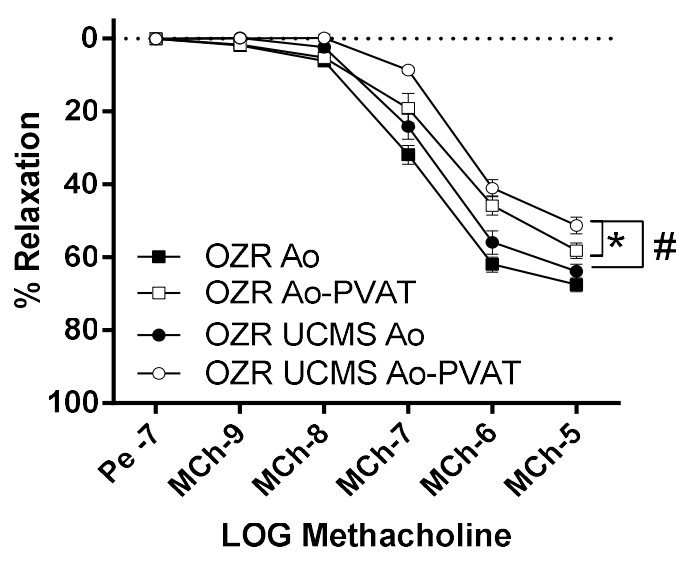

B

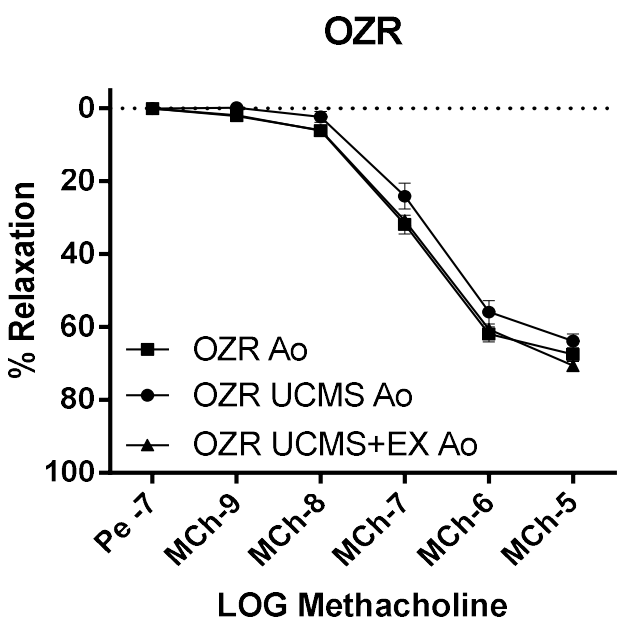

E

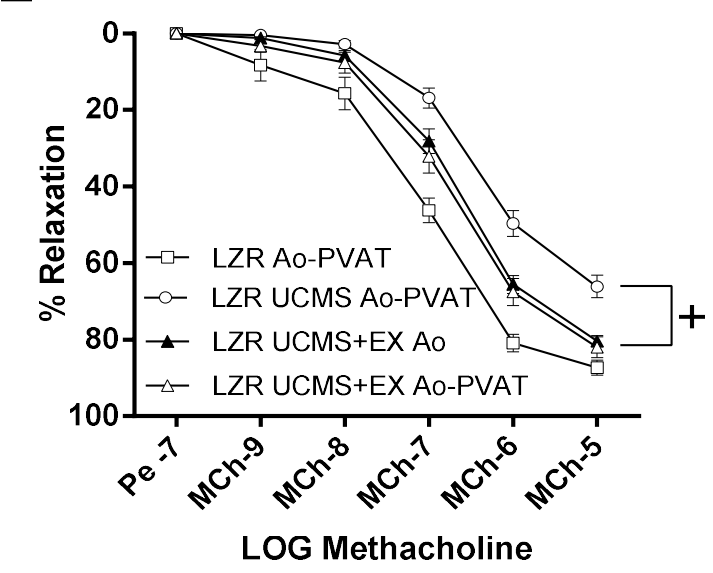

C

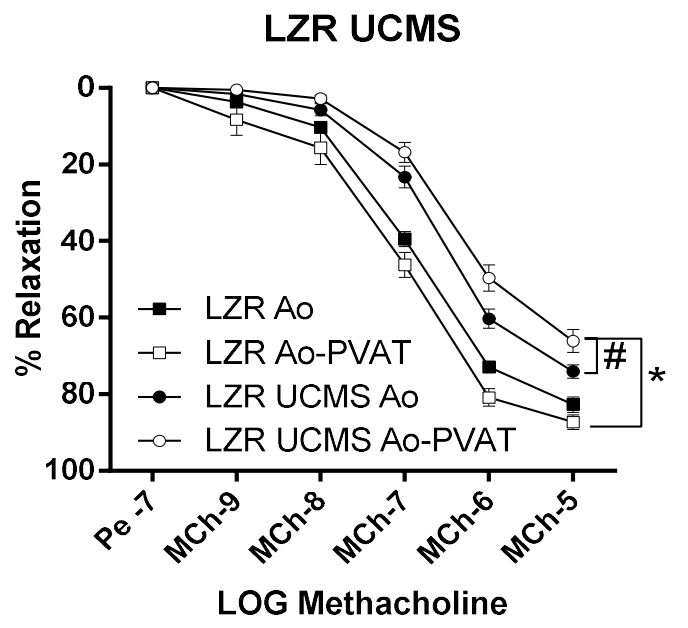

$\mathbf{F}$

OZR UCMS+EX

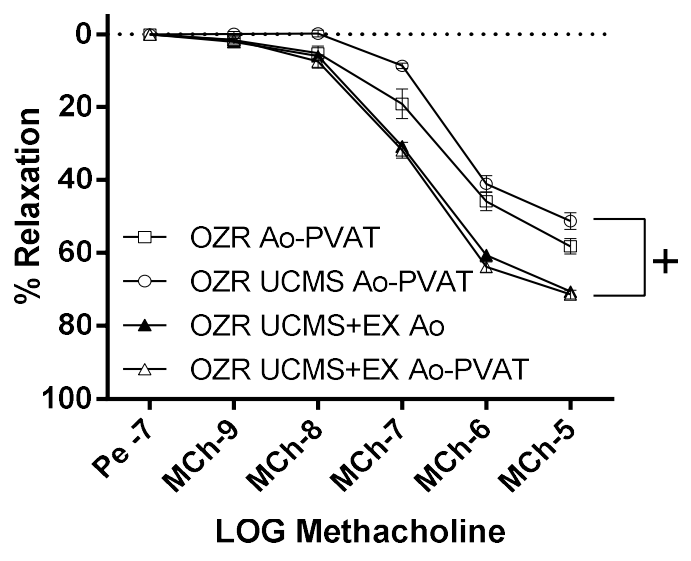


Figure 5.2. Effect of UCMS and Exercise on Nitric Oxide and Aortic Reactive Oxygen Species

A
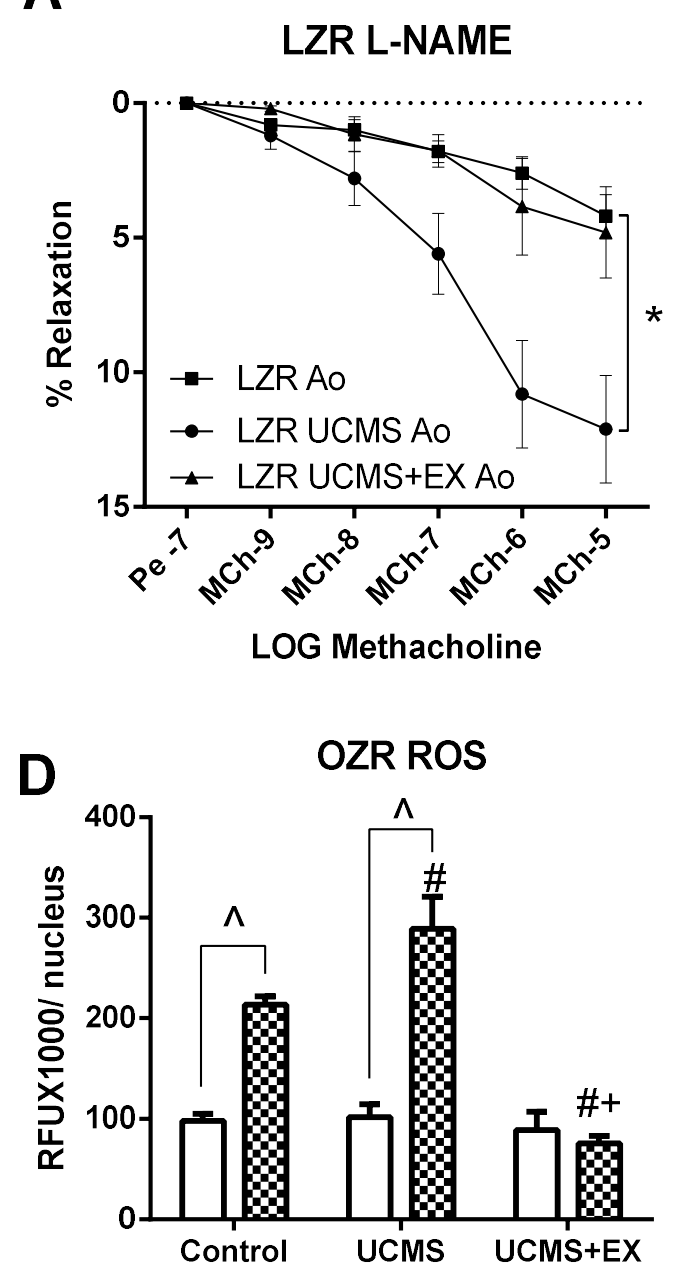

B

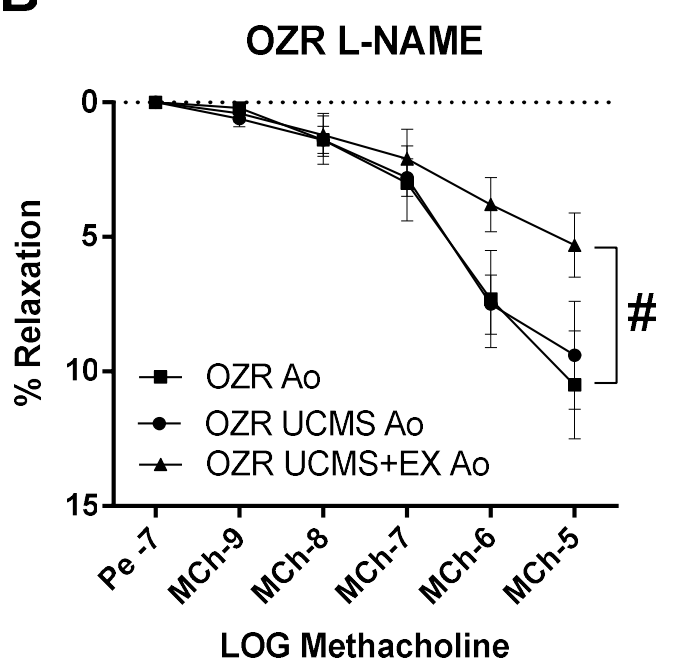

E

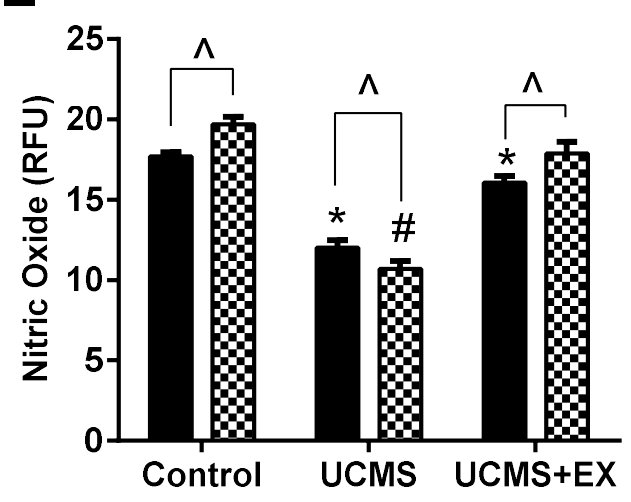

C

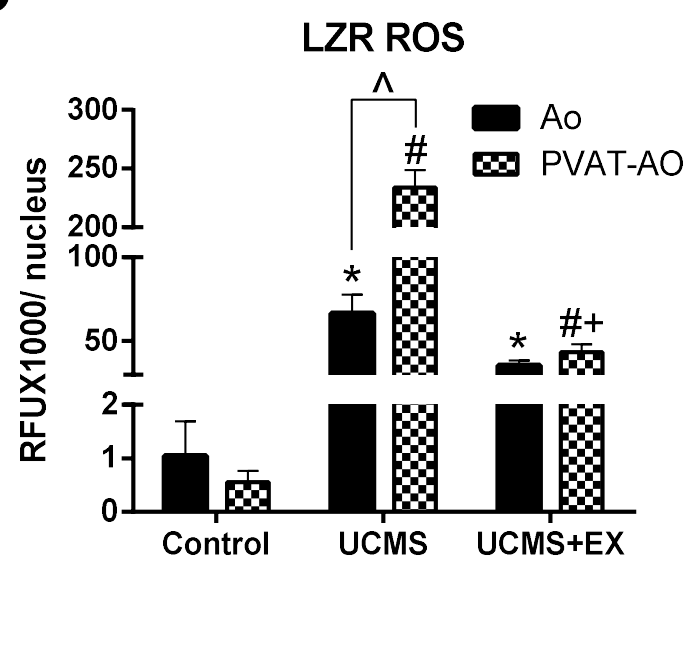

$\mathbf{F}$

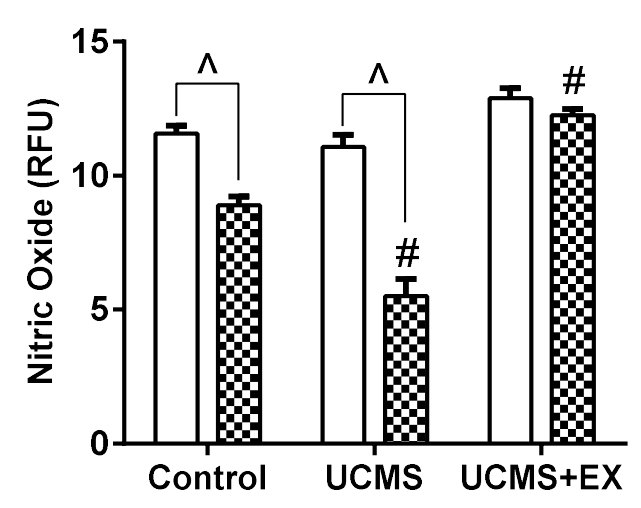


Figure 5.3. Effect of UCMS and Exercise on tPVAT Reactive Oxygen Species and tPVAT Immuno-Attractant Release

A

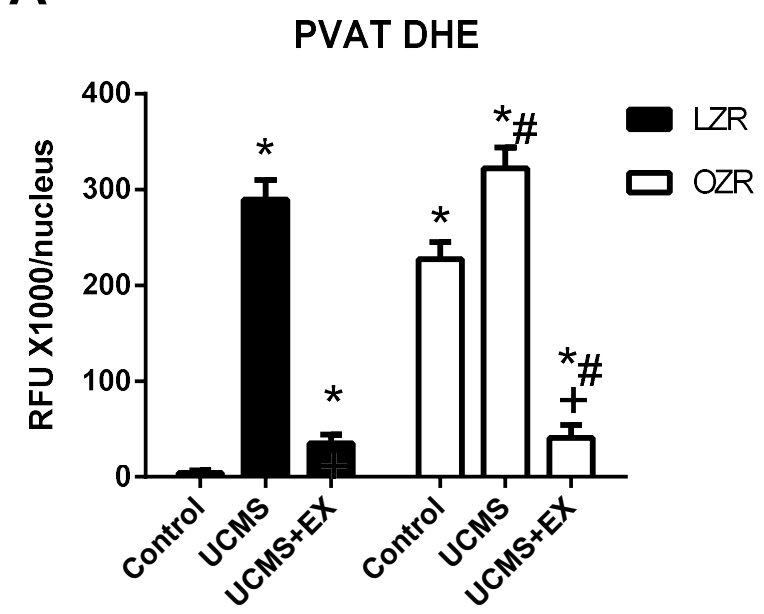

C

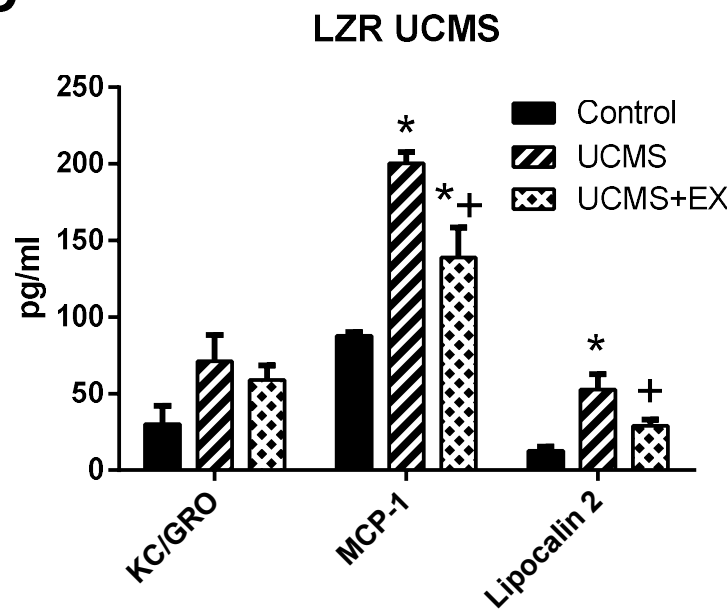

B

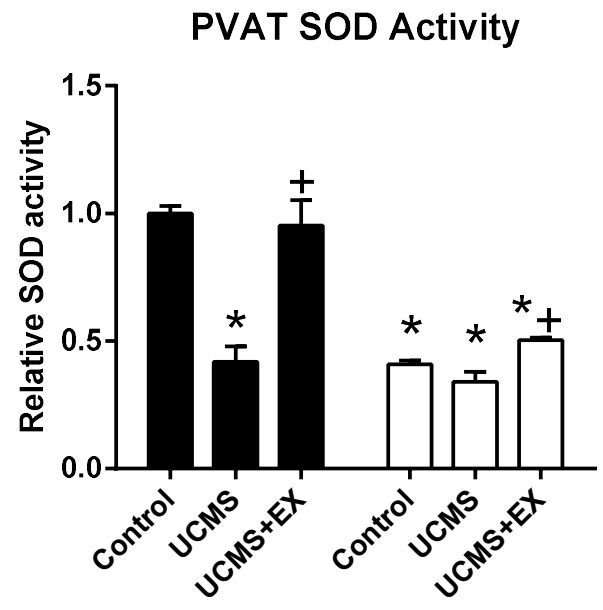

D

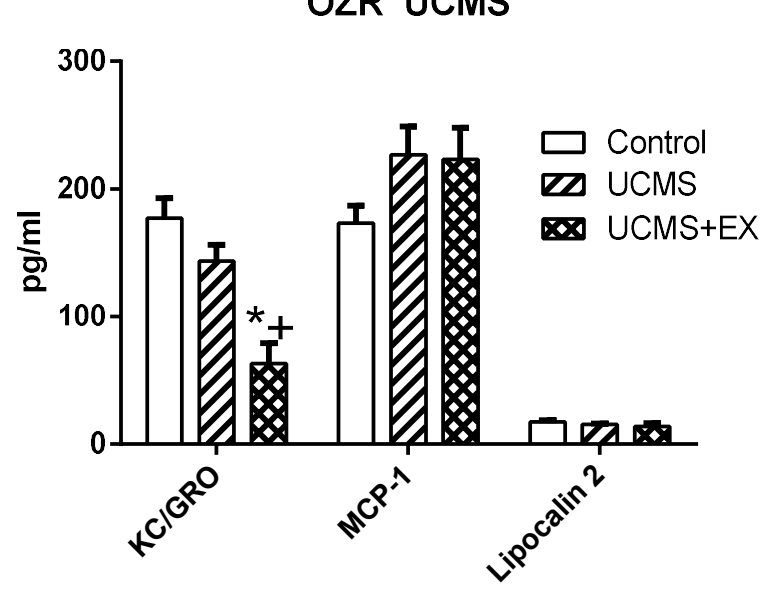


Figure 5.4. Effect of UCMS and Exercise on tPVAT Cytokines

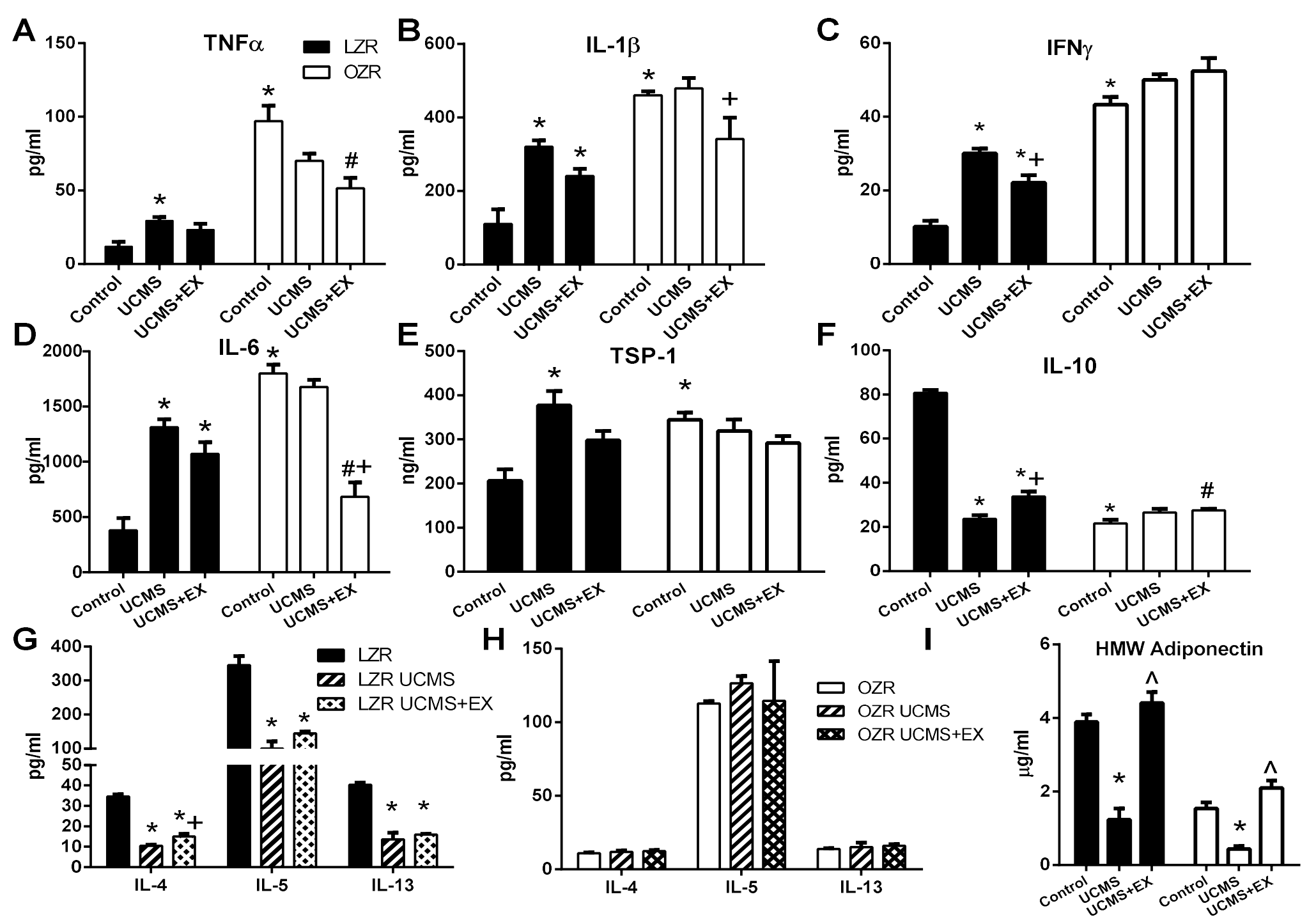


Figure 5.5. Effect of UCMS and Exercise on tPVAT Angiotensin II and Aldosterone

A

A

Angiotensin II

B

Aldosterone
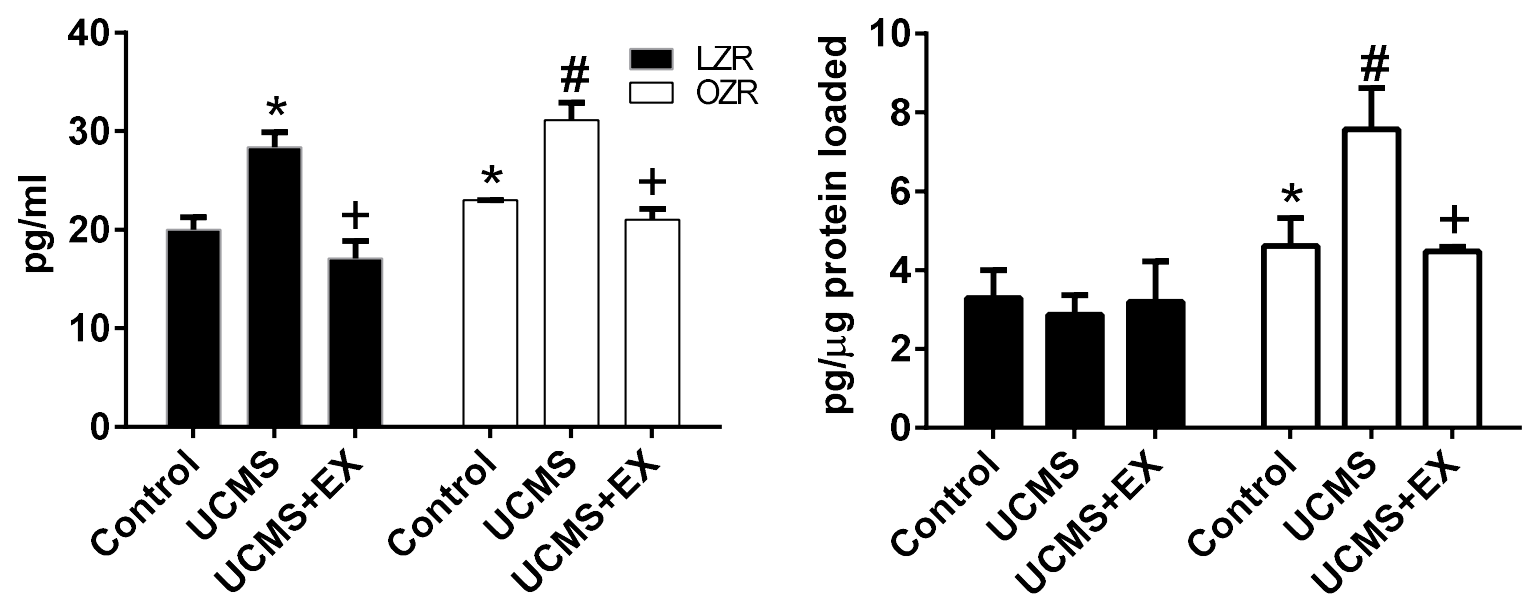

C

ACE2

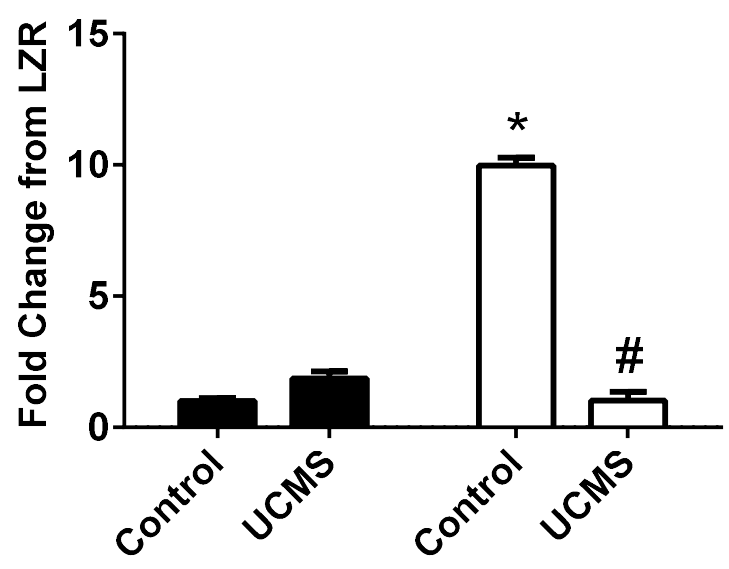


A
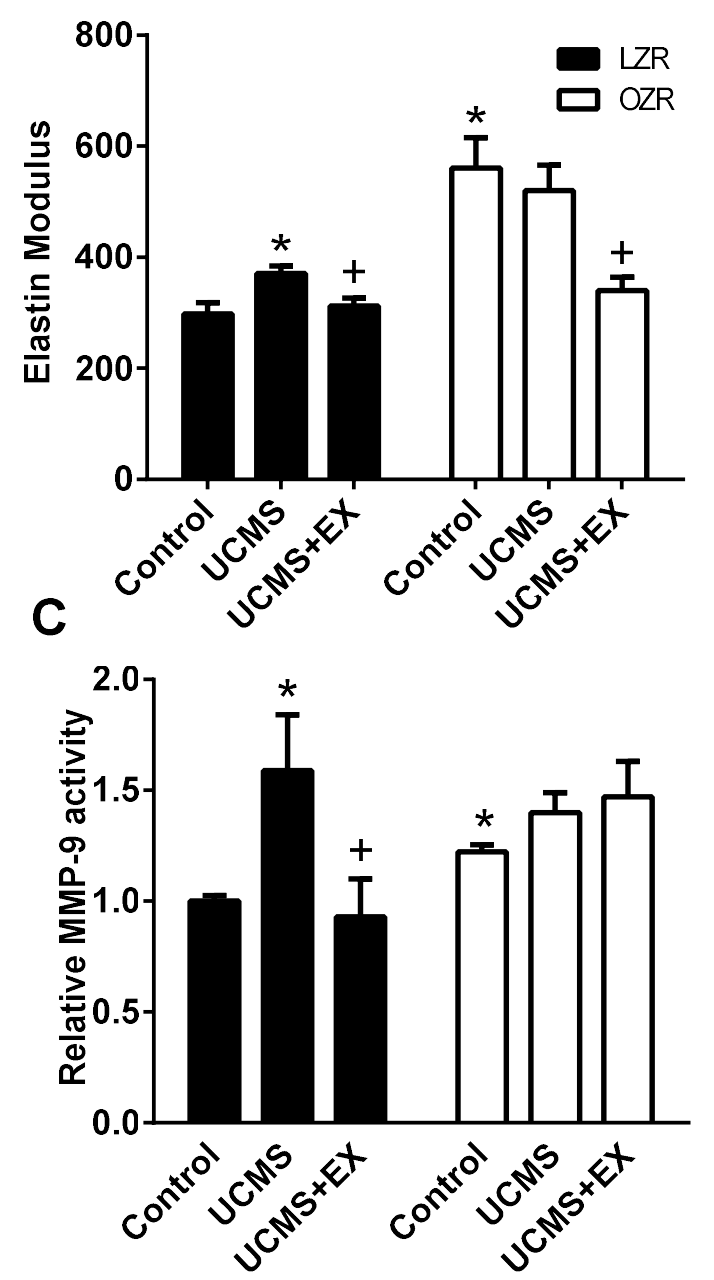

B
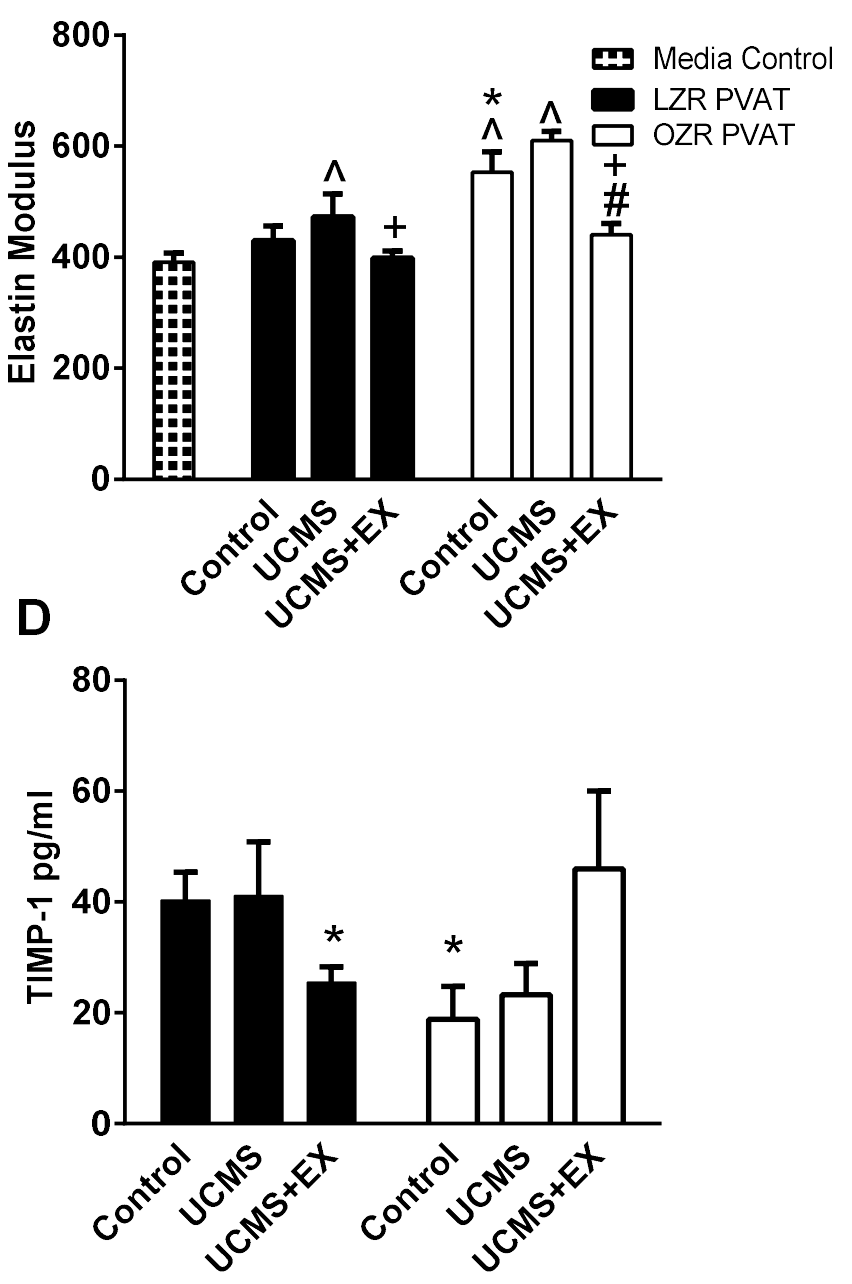


\section{Chapter 6: Dissertation Discussion}

The experiments conducted and represented in this dissertation were in an attempt to illuminate gaps in current knowledge, in the field of disease mediated tPVAT regulation of aortic function. In an attempt to directly define the effect of tPVAT on the aorta a methodology of aortic reactivity was adopted to acquire EDD curves from the same ring with and without the exposure to tPVAT. Similarly, for measurements of NO and ROS production, sequential rings from the same aorta received incubation with tPVAT or no treatment. The methodology used in the dissertation are discussed in detail in chapter 2.

Chapter 3 established the multifaceted effect of TNF $\alpha$ in mediating MetS impairment of tPVAT function with subsequent impairment of aortic EDD and stiffness. Limited work in other labs [13] had started to establish alteration in PVAT function with MetS, which implicated TNF $\alpha$ and NOX ROS. Through the use of TNF $\alpha-n A B$ and NOX2 inhibitor (NOX2ds-TAT) our work builds on this work to establish the increase in tPVAT ROS was mainly mediated through TNF $\alpha$ activation of NOX2. This was supported by similar ROS reducing effects of TNF $\alpha$-nAB and the NOX2 inhibitor (NOX2ds-TAT) with no additive effect with co-treatment, suggesting a common pathway. For the first time, diminished proteasome activity was shown in tPVAT resulting in a buildup of ubiquitinated proteins, which can stimulate of pro-inflammatory cytokine production [4]. Indeed, the cytokine profiles of tPVAT exudate from OZR had increased levels of TNF $\alpha$, TSP-1, IL-6, IL-1 $\beta$, IFN- $\gamma$, MCP-1, and KC/GRO accompanied by a reduction in the antiinflammatory cytokines; adiponectin, IL-4, IL-5, IL-10, and IL-13. This drastic shift in inflammatory profile was, in part, mediated by TNF $\alpha$ activation of NF- $\mathrm{BB}$ in tPVAT. This 
finding was supported when the OZR tPVAT was treated with TNF $\alpha$-nAB resulting in a reduced activation of NF- $\mathrm{KB}$ in tPVAT. We also show TNF $\alpha$ released from OZR tPVAT mediated the activation of aortic ROS, which impaired aortic NO and EDD. Further, TNF $\alpha-n A B$ treated of OZR tPVAT restored aortic ROS, NO, and EDD to levels without tPVAT incubation. These actions of OZR tPVAT were not due to intrinsic aortic properties in the OZR as crossover experiments exposing the aorta from LZR to OZR tPVAT had similar results, which were rescued by treating OZR tPVAT with TNF $\alpha-n A B$. Finally, we examined the stiffness of the aorta and the role of tPVAT on mediating aortic stiffness. Firstly, we measured aortic stiffness (elastin modulus) in freshly isolated aorta's (cleaned from tPVAT) from LZR and OZR's. As expected OZR aortas were significantly stiffer than LZR, which are lab has previously shown to also be the case in humans with MetS [5]. Secondly, we freshly isolated aorta's and using a coculture model, we cultured the isolated aortas with the cleaned from tPVAT from either LZR and OZR to examine the effects of tPVAT on the mechanical properties of the aorta. The aortic stiffness in the OZR appeared to be, in part, mediated by tPVAT as co-culturing LZR aorta with OZR tPVAT significantly elevated the elastin modulus, which was prevented by the treatment of OZR tPVAT with TNF $\alpha-n A B$. These data suggest TNF $\alpha$ may activate tPVAT derived MMP-9 and contribute to aortic stiffness. Additionally, TNF $\alpha$ activation of aortic ROS may also mediate stiffness through elastin fragmentation. The conclusion of this study is signaling through a TNF $\alpha$-NOX2 dependent pathway mediates tPVAT ROS and tissue dysfunction. tPVAT derived TNF $\alpha$ exacerbates aortic dysfunction through activing ROS, reducing NO, impairing EDD, and increasing stiffness. 
The findings in chapter 3 provide evidence for tPVAT derived TNF $\alpha$ in mediating autocrine and paracrine regulation of the aortic dysfunction in MetS. With these data providing evidence for a pathway by which tPVAT mediated aortic dysfunction in MetS, we hypothesize that Ex would prevent the MetS associated impairment of tPVAT. To accomplish this we employed 8 weeks of aerobic exercise training controlling for differences in exercise capacity between LZR and OZR by conducting maximal speed tests. Ex intensity was set at $70 \%$ for 45 minutes following a progressive 15-minute warm up. Importantly, Ex was stopped a minimum of 48 hours prior to terminal procedures to remove the influence of an acute bout of exercise on the collected data. Ex training had no effect on body weight or blood pressure in both LZR-Ex and OZR-Ex. However, the benefits of Ex are known to happen independent of anthropomorphic changes [6, 7]. In tPVAT following Ex, ROS production in OZR-Ex was similar to levels observed in LZR controls. This coincided with a preservation of NO bioavailability in PPAT resulting in higher expression of UCP-1 and lower expression of immune cell markers in OZR-Ex compared to OZR control. This suggested Ex prevented the impairment of NO in APVAT, which maintained a more brown-like phenotype. In turn, the brown-like phenotype helped to suppress ROS production through UCP-1 signaling. Additionally, OZR-Ex presented with an increased global SOD activity, and reduced gene expression of the NOX catalytic subunit GP91 in tPVAT. The lower oxidative load in the tPVAT from OZR-Ex corresponded with greater 19S proteasome cap expression. Additionally, Ex increased concentration of the 20S core in OZR-Ex tPVAT. These data suggested a drastic improvement in proteasome function, and reduced ubiquitin. In OZREx, lower levels of ROS and ubiquitin likely removed two major contributors to increased proinflammatory cytokine production in MetS. Whereby, OZR-Ex had significantly lower concentrations of TNF $\alpha$ and TSP-1 in tPVAT exudate, which likely signifies and important 
benefit of Ex as we showed TNF $\alpha$ mediates vascular impairment in OZR. The tPVAT from OZR-Ex also had increased release of IL-10 and adiponectin, which may aid in the reduction of ROS production and increased NO bioavailability in PVAT.

As expected Ex reduced basal aortic ROS production and improved NO levels in comparison to the OZR control. Additionally, the OZR-Ex tPVAT further reduced aortic ROS production potential through the actions of IL-10 on aortic NOX enzymes. This lower level of ROS production coupled with the NO promoting signals of adiponectin resulted in improved tPVAT mediated EDD in OZR-Ex. Similar results were seen even in the LZR-Ex group as adiponectin was the only cytokine levels to respond to Ex, which likely mediated the improvement in tPVAT mediated EDD. In order to determine if the Ex adaptions in tPVAT were essential for the observed aortic adaptation to Ex we preformed crossover experiments where we exposed OZREx aortic rings to OZR control tPVAT, and tPVAT from OZR controls were treated with the TNF $\alpha-n A B$. These experiments suggested the reduction of TNF $\alpha$ by Ex was essential in promoting improved aortic EDD as OZR-Ex aorta had impaired EDD following incubation with OZR control tPVAT. This effect was absent when OZR control tPVAT was pretreated with a $\mathrm{TNF} \alpha-\mathrm{nAB}$. Further, this may suggest, in general, the function of the aorta was directionally associated with the functional status of the surrounding PVAT. The conclusion from this study was Ex prevented the whitening of tPVAT in MetS, and maintained an antioxidant and antiinflammatory environment. Specifically, reduction of TNF $\alpha$ and higher IL-10 and adiponectin are essential in mediating beneficial Ex adaptions to the aorta further reducing basal ROS and augmenting NO production. This culminated in improved tPVAT mediated EDD and reduced aortic stiffness. 
With Chapter 3 and 4 establishing the effects of MetS and Ex on tPVAT function we were able to then hypothesize on the effect of UCMS alone, UCMS with concurrent MetS, and the effect of Ex on both. Utilizing rodent models of chronic stress, data chapter 5 highlights the role of tPVAT in depressive states alone and concurrently with MetS in mediating aortic impairment. UCMS is a validated method for studying depressive states in rodents [8-10], which was supported in our study by the increased circulating corticosterone, coat scores, and adrenal weights. LZR-UCMS presented with diminished NO and EDD, and an increased production of Ang II and TNF $\alpha$ in LZR-UCMS tPVAT resulting in the autocrine activation of ROS. We believe the UCMS TPVAT resulted in the activation of aortic ROS, reduced NO bioavailability, which further diminished EDD. Additionally, LZR-UCMS had increased aortic stiffness and culturing healthy aortas with LZR-UCMS tPVAT increased aortic stiffness compared to the media control. To the contrary, OZR-UCMS did not alter basal aortic NO or EDD compared to OZR controls, but in the presence of tPVAT, NO and EDD were reduced to a greater magnitude in OZR-UCMS compared to OZR control. However, no change was found in inflammatory cytokine release compared to the already elevated levels in OZR control. The increased aortic impairment by tPVAT in OZR-UCMS was instead likely due to increased release of Ang II and aldosterone, leading to increased activation of aortic ROS. Based on a similar response in LZR and OZR, it appears the increased tPVAT production of Ang II, and decreased production of adiponectin are common responses to UCMS independent of disease state. It has been previously shown that Ang II can upregulate pro-inflammatory cytokines and aldosterone; however, divergent actions based on MetS status were observed in tPVAT. In LZR-UCMS, increased Ang II with subsequent upregulation of pro-inflammatory cytokines were likely culprits of the 
whitening phenotype and a mediator of aortic dysfunction. While in OZR-UCMS, increased Ang II resulted in elevated aldosterone with no effect on pro-inflammatory cytokines.

We next examined the role of exercise training on ameliorating the tPVAT dysfunction observed in UCMS groups. In LZR- and OZR-UCMS+Ex groups, the aortic dysfunction which was mediated by tPVAT was prevented and reflected higher production of NO and increased EDD by tPVAT compared to LZR- and OZR-UCMS groups. The major effect of Ex appeared to be on Ang II and adiponectin, whereby lower Ang II and higher adiponectin levels in tPVAT were noted in the LZR- and OZR-UCMS+Ex groups vs. their UCMS controls. However, Ex also reduced aldosterone, IL- $1 \beta$ and IL- 6 in OZR-UCMS+Ex and IFN- $\gamma$ and TSP-1 in LZRUCMS+Ex, which suggested Ex was able to prevent the MetS triggered inflammation and UCMS activation of the local RAAS.

Ex also had beneficial effects on aortic stiffness. The tPVAT mediated aortic stiffness in the LZR-UCMS was prevented in LZR-UCMS+Ex group, and was further demonstrated by the respective effects on TPVAT MMP-9 activity. UCMS had no added effect on the already elevated aortic stiffness in OZR; however, in the OZR-UCMS+Ex group, aortic stiffness was reduced, and tPVAT from this group did not induce aortic stiffness in the co-culture experiments. These data suggest the activation of tPVAT inflammatory cytokines triggers aortic stiffness likely through the activation of MMP-9. Accordingly, the anti-inflammatory actions of Ex prevent the MetS and UCMS mediated aortic stiffness. 


\section{Summary}

The key findings of this work are OZR tPVAT dysfunction through a TNF $\alpha-N O X 2$ dependent pathway regulate aortic activation of ROS, impairing EDD. Additionally, $\mathrm{TNF} \alpha$, in part, mediates OZR tPVAT stiffening of the aorta through increased MMP9 activity. For the first time in tPVAT we show Mets causes proteasome dysfunction, which may act as a trigger for inflammation. The exposure of LZR to UCMS caused a phenotypic shift and as such the tPVAT in the LZR-UCMS group resembled tPVAT from OZR controls (i.e., increased ROS, proinflammatory cytokines, and hormones). This was coupled with tPVAT increased activation of aortic ROS, impaired EDD, and aortic stiffness. The comorbid state of OZR with UCMS did not result in any basal change in aortic function or pro-inflammatory cytokines in tPVAT. However, OZR-UCMS did present with elevated Ang II and aldosterone, which activated aortic ROS and diminished EDD significantly more than just OZR alone. Finally, the detrimental actions of MetS, UCMS and UCMS in combination with MetS on tPVAT function and tPVAT impairment of the aorta were prevented by Ex. Additionally we showed for the MetS impairment of proteasome function in PVAT was prevented by Ex. This work establishes the essential role of tPVAT in conferring the beneficial effects of Ex on the aorta. Detailed comparison of the effects of the various experimental groups on tPVAT (Table 1) and tPVAT regulation of the aorta (Table 2) can be found below. 
Table 6.1. Effect of tPVAT on Aortic Function

\begin{tabular}{|c|c|c|c|c|c|c|c|c|c|c|}
\hline \multicolumn{3}{|c|}{ Table 1} & \multicolumn{8}{|c|}{ tPVAT Factors } \\
\hline $\begin{array}{l}\text { Experimentala } \\
\text { Grout }\end{array}$ & $\begin{array}{c}\text { Compared } \\
\text { to }\end{array}$ & $\begin{array}{l}\text { tPVAT } \\
\text { ROS }\end{array}$ & TNF $\alpha$ & $\mathrm{HL}-1 \mathrm{\beta}$ & IFN-y & TSP-1 & $\mathrm{IL}-10$ & $\begin{array}{l}\text { HimW } \\
\text { Adiponectin }\end{array}$ & Ang II & Aldosterone \\
\hline LZR UCMS & LZR control & \} & $\widehat{\}}$ & $\widehat{\mho}$ & & 1 & & & 1 & $\Leftrightarrow$ \\
\hline LZR Ex & LRR control & $<$ & $\Leftrightarrow$ & $\Leftrightarrow$ & $\Leftrightarrow$ & $\Leftrightarrow$ & $\Leftrightarrow$ & 个 & $\mathrm{N} / \mathrm{A}$ & $\mathrm{N} / \mathrm{A}$ \\
\hline \multirow{2}{*}{ LZR UCMS+Ex } & LZR control & $\widehat{1}$ & $\Leftrightarrow$ & $\hat{\imath}$ & $\hat{1}$ & $\Leftrightarrow$ & & $\Leftrightarrow$ & $\Leftrightarrow$ & $\Leftrightarrow$ \\
\hline & LZR UCMS & S & $\Leftrightarrow$ & $\Leftrightarrow$ & $\Omega$ & $\Leftrightarrow$ & 仓 & \} & ภ & $\Leftrightarrow$ \\
\hline OzR control & LZR control & 个 & 个 & $\hat{\imath}$ & $\hat{\imath}$ & & 几 & ת & $\widehat{1}$ & $\widehat{1}$ \\
\hline оzв uсмs & OZR control & $\Leftrightarrow$ & $\Leftrightarrow$ & $\Leftrightarrow$ & $\Leftrightarrow$ & $\Leftrightarrow$ & $\Leftrightarrow$ & $\Omega$ & 个 & 个 \\
\hline OZREx & OZR control & $\Omega$ & ת & $\Leftrightarrow$ & $\Leftrightarrow$ & $\Omega$ & 个 & $\widehat{\widehat{1}}$ & $\mathrm{~N} / \mathrm{A}$ & N/A \\
\hline \multirow{2}{*}{ OZR UCMS+Ex } & OZR control & $\Omega$ & $\Omega$ & $\Leftrightarrow$ & $\Leftrightarrow$ & $\Leftrightarrow$ & 仓 & $\Leftrightarrow$ & $\Leftrightarrow$ & $\Leftrightarrow$ \\
\hline & OzR UCMS & $\Omega$ & $\Leftrightarrow$ & ת & $\Leftrightarrow$ & $\Leftrightarrow$ & $\Leftrightarrow$ & $\widehat{\imath}$ & $\Omega$ & $\Omega$ \\
\hline
\end{tabular}

Arrows represent direction of change in tPVAT of the "experimental group" from the "compared to". Red indicates detrimental change while green represents beneficial change, and blue is neutral. ROS, reactive oxygen species; TNF $\alpha$, tumor necrosis factor alpha; IL, interleukin; IFN$\gamma$, interferon gamma; TSP, thrombospondin; HMW, high molecule weight; Ang, angiotensin; UCMS, unpredictable chronic mild stress; Ex, aerobic exercise training. 
Table 6.2. Effect of tPVAT on Aortic Function

\begin{tabular}{|c|c|c|c|c|c|}
\hline \multicolumn{2}{|c|}{ Table 2} & \multicolumn{4}{|c|}{ tPVAT Effect on Aorta } \\
\hline $\begin{array}{l}\text { Experimental } \\
\text { Group }\end{array}$ & $\begin{array}{c}\text { Compared } \\
\text { to }\end{array}$ & Aortic ROS & NO & EDD & Stiffness \\
\hline \multirow{2}{*}{ LZR UCMS } & $\begin{array}{l}\text { Ao w/o } \\
\text { tPVAT }\end{array}$ & & & & \\
\hline & LZR control & & & & \\
\hline \multirow{2}{*}{ LZR Ex } & $\begin{array}{l}\text { Ao w/o } \\
\text { tPVAT }\end{array}$ & & & & $\mathrm{N} / \mathrm{A}$ \\
\hline & LZR control & & & & N/A \\
\hline \multirow{3}{*}{ LZR UCMS+Ex } & $\begin{array}{l}\text { Ao w/o } \\
\text { tPVAT }\end{array}$ & & & & \\
\hline & LZR control & & & & \\
\hline & LZR UCMS & & & & \\
\hline \multirow{2}{*}{ OZR control } & $\begin{array}{l}\text { Ao w/o } \\
\text { tPVAT }\end{array}$ & & & & \\
\hline & LZR control & & & & \\
\hline \multirow{2}{*}{ OZR UCMS } & $\begin{array}{l}\text { Ao w/o } \\
\text { tPVAT }\end{array}$ & & & & \\
\hline & $\begin{array}{c}\text { OZR } \\
\text { control }\end{array}$ & & & & \\
\hline \multirow{2}{*}{ OZR Ex } & $\begin{array}{l}\text { Ao w/o } \\
\text { tPVAT }\end{array}$ & & & & $N / A$ \\
\hline & $\begin{array}{c}\text { OZR } \\
\text { control }\end{array}$ & & & & $N / A$ \\
\hline \multirow{3}{*}{ OZR UCMS+EX } & $\begin{array}{l}\text { Ao w/o } \\
\text { tPVAT }\end{array}$ & & & & \\
\hline & $\begin{array}{c}\text { OZR } \\
\text { control }\end{array}$ & & & & \\
\hline & OZR UCMS & & & & \\
\hline
\end{tabular}

Arrows represent direction of change in TPVAT of the "experimental group" from the "compared to". Red indicates detrimental change while green represents beneficial change, and blue is neutral. ROS, reactive oxygen species; NO, nitric oxide; EDD, endothelial dependent dilation. Ao w/o tPVAT represents comparison to the aorta from the respective experimental group without incubation with tPVAT. 


\section{Limitations}

There are a number of limitations with regard to the studies conducted. One limitation of the study design is the implantation of the Ex protocol during the development of MetS and depressive states. As such the results should be interpreted as more of a preventative role of Ex. However, previous work from our lab shows inflammation and vascular dysfunction is already present at the age rats were enrolled in the study. As for the effect of Ex on UCMS measures of coat score and adrenal weights were unaffected by Ex. This suggests Ex did not blunt the stressful stimulus, however we cannot rule out the possibility of Ex dampening the SNS response to stress as this is a well-known effect of Ex.

A second limitation is with the co-culture experiments. Flow is an important stimulus for NO production in arteries and as described in the introduction of this dissertation, NO is an important mediator of aortic stiffness. To address this in the experimental design the use of a media only control should account for the increase in stiffness due to lack of flow. What is lost in this experiment is the contribution of tPVAT inhibition of NO levels on aortic stiffness. Further, the longitudinal and circumferential components of aortic stiffness are lost in this experimental design. However, evidence suggests the test is a good metric of overall conduit vessel stiffness [11]. Additionally, the results are limited as collagen, collagen crosslinks, and elastin were not directly analyzed.

Another potential limitation is the removal of tPVAT may reduce the influence of certain mediators on the aorta. While this is a strength of the study because it allows us to directly test the impact of tPVAT through conducting basal and tPVAT incubations on the same ring. The 
methodology may limit the effect of lipid mediators, exosome, and short-lived mediators due to distance, water solubility, and density. However, the vast majority of significant vascular modulators from tPVAT are cytokines or long-lived radicals which should be unaffected by the methodology employed.

Another minor limitation is the use of the DHE assay, which detects both superoxide and hydrogen peroxide, which cannot be differentiated between when analyzing by imaging software. Analysis by HPLC can distinguish between the production of superoxide and hydrogen peroxide, but the technology was unavailable at the time of the project.

A final potential limitation is the use of the OZR rat. The leptin receptor mutation inhibits all leptin signaling, which shown to play some role in PVAT function. However, the OZR is a clinically relevant model of MetS as clinical patients normally present with leptin signaling resistance.

\section{Clinical prospective}

Clinically this works adds to our knowledge of the MetS induced aortic pathology. Highlighting the role of local inflammation in activation of endothelial ROS. This work suggests modifying the function of the surrounding PVAT may mediate vascular benefits. Suggesting employing treatments directed at PVAT may be successful in restoring vascular function. This highlight in chapter 4 as Ex improved aortic function was dependent on alterations to the surrounding tPVAT. A few targets are presented for potential treatment options. First, from this work and others developing pharmaceuticals directed at PVAT resident NOX2 expressing immune cells 
may have promising implications are it appears these cells are essential in MetS induce PVAT dysfunction. Further this work elucidates mechanism through which exercise mediates vascular protection.

\section{$\underline{\text { Future Direction }}$}

A simple follow up experiment to the ones conducted in this dissertation could be to implement Ex after rats had reached full progression of MetS and additionally after the onset of a depressive state. This would allow us to test the efficacy of Ex in reversing the effects of MetS and UCMS on tPVAT outcomes. Another logical progression from the work presented here in this dissertation would be to implement drug treatment to alter components of tPVAT dysfunction or MetS components. Our lab has previously employed this approach in assessment of cerebral vascular function in MetS [12]. This approach of in-vivo drug treatment may further identify the mechanisms through which MetS and UCMS causes impairment of tPVAT function. The work in this dissertation showed TNFa is responsible for MetS impairment of tPVAT, to build on these findings evaluation of TNF $\alpha$ signaling pathways leading to the observed outcomes would be warranted. In addition to the approaches used in this dissertation flow cytometry analysis of tPVAT would further illuminate mechanisms of tPVAT dysfunction through the population profile of immune cells. Flow cytometry analysis coupled with drug treatments may uncover important disease associated signals leading to the observed dysfunction in this dissertation.

The majority of PVAT experiments, including the ones conducted in this dissertation, are conducted ex-vivo. While ex-vivo experiments have greatly expanded our knowledge of PVAT function and regulation of the underlying vessel, studies evaluating PVAT in-vivo are needed. 
However, this presents with a number of challenges. Genetic knockout animal lines represent an important means to identify physiologic function, especially those which knockouts factors in a tissue specific manner. These tissue specific knock models allow you to conduct loss of function studies while reducing systemic influences from whole body knockouts. However, in the context of PVAT methodological hurdles remain as identification of a PVAT specific drive have not been identified. The development/ identification of PVAT specific promoters would allow for greater interrogation of PVAT signaling in-vivo. Further combining in-vivo manipulation of tPVAT with in-vivo assessment of aortic function (i.e. pulse wave velocity) would generate more physiologically relevant experiments. For example, a logical progression from this work may to be to generate a PVAT knockout of TNF $\alpha$ along with an inducible PVAT TNF $\alpha$ knockout to examine the role of adipocyte regenerated $\mathrm{TNF} \alpha$ in regulation of aortic function and the progression of MetS induced PVAT dysfunction. While identification of a PVAT adipocyte specific promoter remains elusive, modulation of immune cells represents an alternative approach. Immune cell infiltration is recognized as an important mechanism in PVAT regulation of disease progression. Generation of immune cell specific knockouts are a useful alternative to study their role in PVAT dysfunction. Recently, this approach has been implemented to study the effects of hypertension on PVAT function [13].

Similar to the lack of PVAT specific knockouts the field is currently lacking a PVAT specific cell line. Currently, cell work for PVAT is being conducted in white adipose cell lines, which as discussed in chapter 1 develop from different stem cell origins and display different phenotypes. The development of a PVAT cell line would allow for a more appropriate assessment of PVAT adipocyte function. For application to our current line of research we could assess time course 
responses of adipocyte to various MetS associated stimuli. For example, assess PVAT adipocyte phenotype, ROS, TNF $\alpha$, and adiponectin in response to high glucose and high free fatty acids. This would allow for a better understanding of the individual and combined effects of MetS on

PVAT adipocytes. Additionally, using our animal models and interventions tPVAT immune cells could be isolated and used in a co-culture experiment to better understand the effects of the infiltrated immune cell population on PVAT adipocyte function.

Our data showed TSP-1 was elevated in MetS and restored with exercise. TSP-1 is known to regulate a number of vascular pathways. TSP-1 negatively regulates capillarity and may play a role in developing tPVAT dysfunction. TSP-1 inhibition of eNOS could alter adipose phenotype and directly impact the function of the underlying blood vessel. Finally, TSP-1 has been implicated in the regulation of vascular stiffening through its actions on the ECM. Additionally, TSP-1 is partially regulated by TNF $\alpha$ potentially linking its expression to the increased inflammation in tPVAT. The multifaceted actions of TSP-1 warrant further investigation in tPVAT to determine its role in the TNF $\alpha$ dependent signaling shown to mediate tPVAT impairment of the aorta.

\section{Long Term Outlook}

Logistical hurdles remain in the study of tPVAT. In addition to the identification and development of PVAT adipocyte specific promoters and cell lines; implementation of in-vivo assessment of cytokine levels through probes, catheters, or live image tracking would prove beneficial in understanding the in-vivo relationship between the aorta and the PVAT. Clearing 
these technical hurdles would open the way for a myriad of experimental options to expand PVAT research.

Outside of technological advancement I believe the development of time course studies to elucidate the development of PVAT dysfunction with disease will drive the field forward. Time courses to understand the early signaling cascades, which lead to dysfunction may yield novel therapeutic targets for the prevention of vascular disease. Additionally, PVAT is densely innervated understanding the nerve to PVAT crosstalk and alteration with various disease states would add another layer of understanding to the function of PVAT and its regulation of the vasculature.

\section{$\underline{\text { References: }}$}

1. Marchesi, C., et al., Endothelial nitric oxide synthase uncoupling and perivascular adipose oxidative stress and inflammation contribute to vascular dysfunction in a rodent model of metabolic syndrome. Hypertension, 2009. 54(6): p. 1384-92.

2. Greenstein, A.S., et al., Local inflammation and hypoxia abolish the protective anticontractile properties of perivascular fat in obese patients. Circulation, 2009. 119(12): p. 1661-70.

3. Virdis, A., et al., Tumour necrosis factor-alpha participates on the endothelin-1/nitric oxide imbalance in small arteries from obese patients: role of perivascular adipose tissue. Eur Heart J, 2015. 36(13): p. 784-94. 
4. Ghosh, A.K., et al., Elevated Endoplasmic Reticulum Stress Response Contributes to Adipose Tissue Inflammation in Aging. J Gerontol A Biol Sci Med Sci, 2015. 70(11): p. 1320-9.

5. Donley, D.A., et al., Aerobic exercise training reduces arterial stiffness in metabolic syndrome. J Appl Physiol (1985), 2014. 116(11): p. 1396-404.

6. Balducci, S., et al., Changes in physical fitness predict improvements in modifiable cardiovascular risk factors independently of body weight loss in subjects with type 2 diabetes participating in the Italian Diabetes and Exercise Study (IDES). Diabetes Care, 2012. 35(6): p. 1347-54.

7. Balducci, S., et al., Anti-inflammatory effect of exercise training in subjects with type 2 diabetes and the metabolic syndrome is dependent on exercise modalities and independent of weight loss. Nutr Metab Cardiovasc Dis, 2010. 20(8): p. 608-17.

8. Mineur, Y.S., C. Belzung, and W.E. Crusio, Effects of unpredictable chronic mild stress on anxiety and depression-like behavior in mice. Behav Brain Res, 2006. 175(1): p. 43 50.

9. Willner, P., Validity, reliability and utility of the chronic mild stress model of depression: a 10-year review and evaluation. Psychopharmacology (Berl), 1997. 134(4): p. 319-29.

10. Willner, P., et al., Reduction of sucrose preference by chronic unpredictable mild stress, and its restoration by a tricyclic antidepressant. Psychopharmacology (Berl), 1987. 93(3): p. 358-64.

11. Humphrey, J.D., Cardiovascular solid mechanics : cells, tissues, and organs. 2002, New York: Springer. xvi, $757 \mathrm{p}$. 
12. Brooks, S.D., et al., Metabolic syndrome impairs reactivity and wall mechanics of cerebral resistance arteries in obese Zucker rats. Am J Physiol Heart Circ Physiol, 2015. 309(11): p. H1846-59.

13. Mikolajczyk, T.P., et al., Role of chemokine RANTES in the regulation of perivascular inflammation, T-cell accumulation, and vascular dysfunction in hypertension. FASEB J, 2016. 30(5): p. 1987-99. 